\author{
UCRL-ID-111623
}

\title{
ANALYSIS AND EVALUATION OF VOC Removal Technologies Demonstrated AT SAVANNAH RIVER
}

Dwayne A. Chesnut, Jeffrey Wagoner, John J. Nitao, Sierra Boyd, Ronald J. Shaffer, Edward J. Kansa, and Thomas A. Buscheck

Lawrence Livermore National Laboratory

Karsten Pruess, Lawrence Berkeley Laboratory

Ronald W. Falta, Clemson University

SEPTEMBER 1993

\section{MASTER}




\section{CONTENTS}

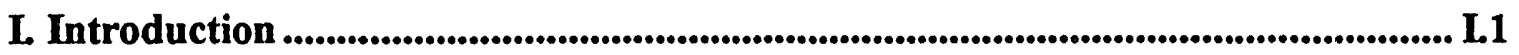

Full Scale Pump and Treat ........................................................................................ I.3

Vertical Well SVE Test........................................................................................................ 1.4

Horizontal Well ISAS Test ...................................................................................... 1.5

Performance Comparisons .............................................................................................. $\mathrm{L} .6$

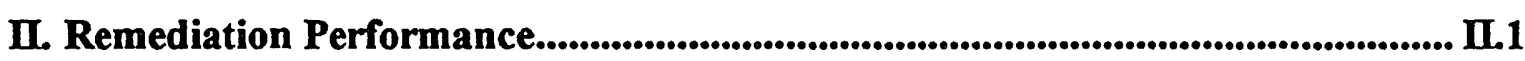

Ideal System ........................................................................................................................... II.1

Real Systems .................................................................................................................. II.1

Objectives of Analysis \& Evaluation........................................................................... II.5

III. Effects of Flow Geometry .................................................................................... II.1

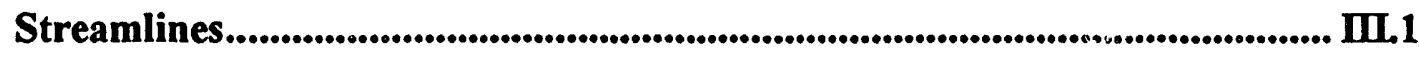

Streamline Models for Vertical Vapor Extraction Wells.................................... III.3

IV. Effects of Permeability Variation ............................................................................... IV.1

Heterogeneity Parameter Models............................................................................ IV.2

Application to SR In Situ Air Stripping Test .................................................. IV.9

Application to SR Pump and Treat Data........................................................ IV.17

V. Field Data................................................................................................................................ V.1

Well Completions.................................................................................................................... V.1

Contaminant Concentrations....................................................................................... V.3

Core Sample Permeabilities....................................................................................... V.4

Pressure Response: Vertical Well Vacuum Extraction Test ............................ V.7

Tracer Test............................................................................................................................ V.12

VI. Geologic and Geophysical Analyses .............................................................................. VI.1

Stratigraphic Correlations.......................................................................... VI.1

Geophysical Analyses............................................................................................. VI.24

VII. Simulation Studies........................................................................................................... VII.1

VIII. Conclusions and Recommendations ..................................................................... VIII.1 


\section{CONTENTS (CONTINUED)}

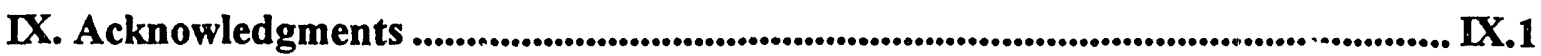

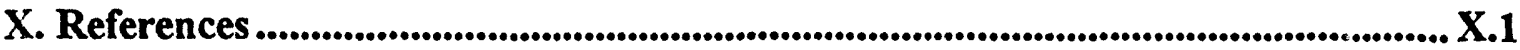

Appendix A. Streamline Models for Vertical Vapor Extraction Wells and Horizontal Injection and Extraction Wells........................................................................... A-1

Analytical Modeling of Advective Contaminant Transport During Soil

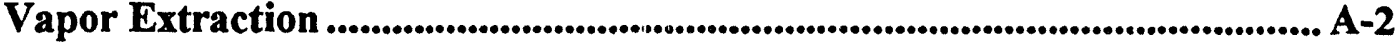

Analytical Solutions for Gas Flow Due to Gas Injection and Extraction from Horizontal Wells .................................................................. A-4

Appendix B: Heterogeneity Parameter Models ....................................................... B-1

Waterflooding ................................................................................................................. B-1

Vapor Extraction ........................................................................................................... B-9

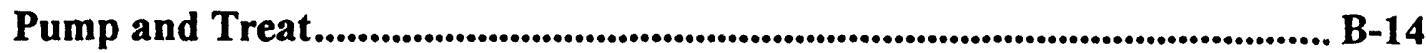

Appendix C: Fitting Heterogeneity Parameter Models to Field Data........................ C-1

Benton Waterflood ....................................................................................................... C-1

Savannah River In-Situ Air Stripping Data.................................................... C-3

Pump and Treat Data from Savannah River..................................................... C-7

Appendix D. Analysis of Flow Processes During TCE Infiltration in Heterogeneous Soils at the Savannah River Site, Aiken, South Carolina. 


\section{ANALYSIS AND EVALUATION OF VOC REMOVAL TECHNOLOGIES DEMON'STRATED AT SAVANNAH RIVER}

\section{INTRODUCTION}

Volatile Organic Compounds, or VOCs, zre ubiquitous subsurface contaminants at industrial as well as DOE sites. At the Savannah River Plant (see Figure I.1), the principle VOCs contaminating the subsurface below A-Area and $\mathrm{M}$-Area are Trichloroethylene $\left(\mathrm{C}_{2} \mathrm{HCl}_{3}\right.$, or TCE) and Tetrachloroethylene $\left(\mathrm{C}_{2} \mathrm{Cl}_{4}\right.$, or PCE). These compounds were used extensively as degreasing solvents from 1952 until 1979, and the waste solvent which did not evaporate (on the order of $2 \times 10^{6}$ pounds) was discharged to a process sewer line leading to the M-Area Seepage Basin (Figure I.2). These compounds infiltrated into the soil and underlying sediments from leaks in the sewer line and elsewhere, thereby contaminating the vadose zone between the surface and the water table as well as the aquifer.

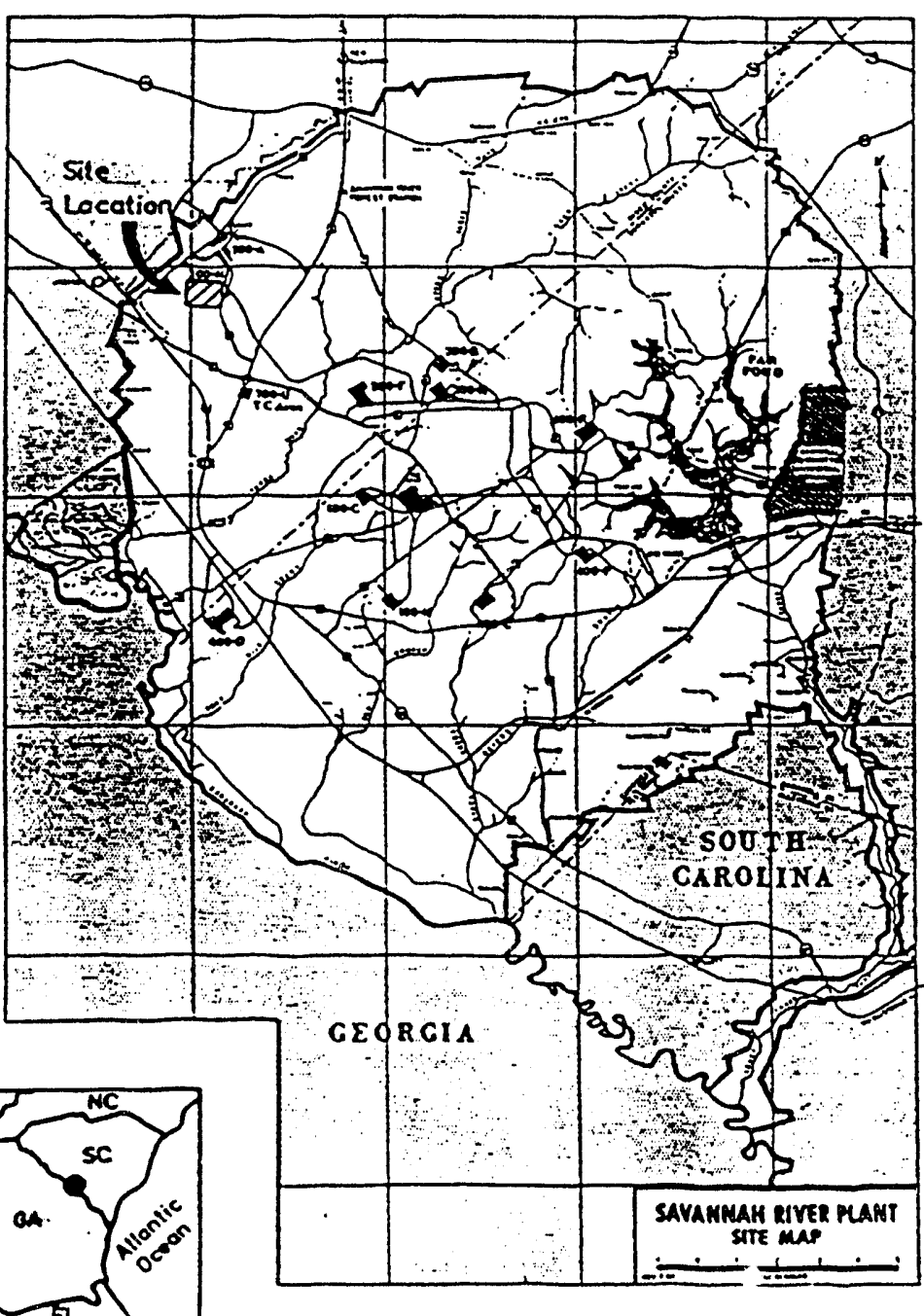

Figure I.1. Location Map for the Savannah River Site [from Fig. 2.1, Kaback et al., WSRC-RP-89-784 (1989)] 


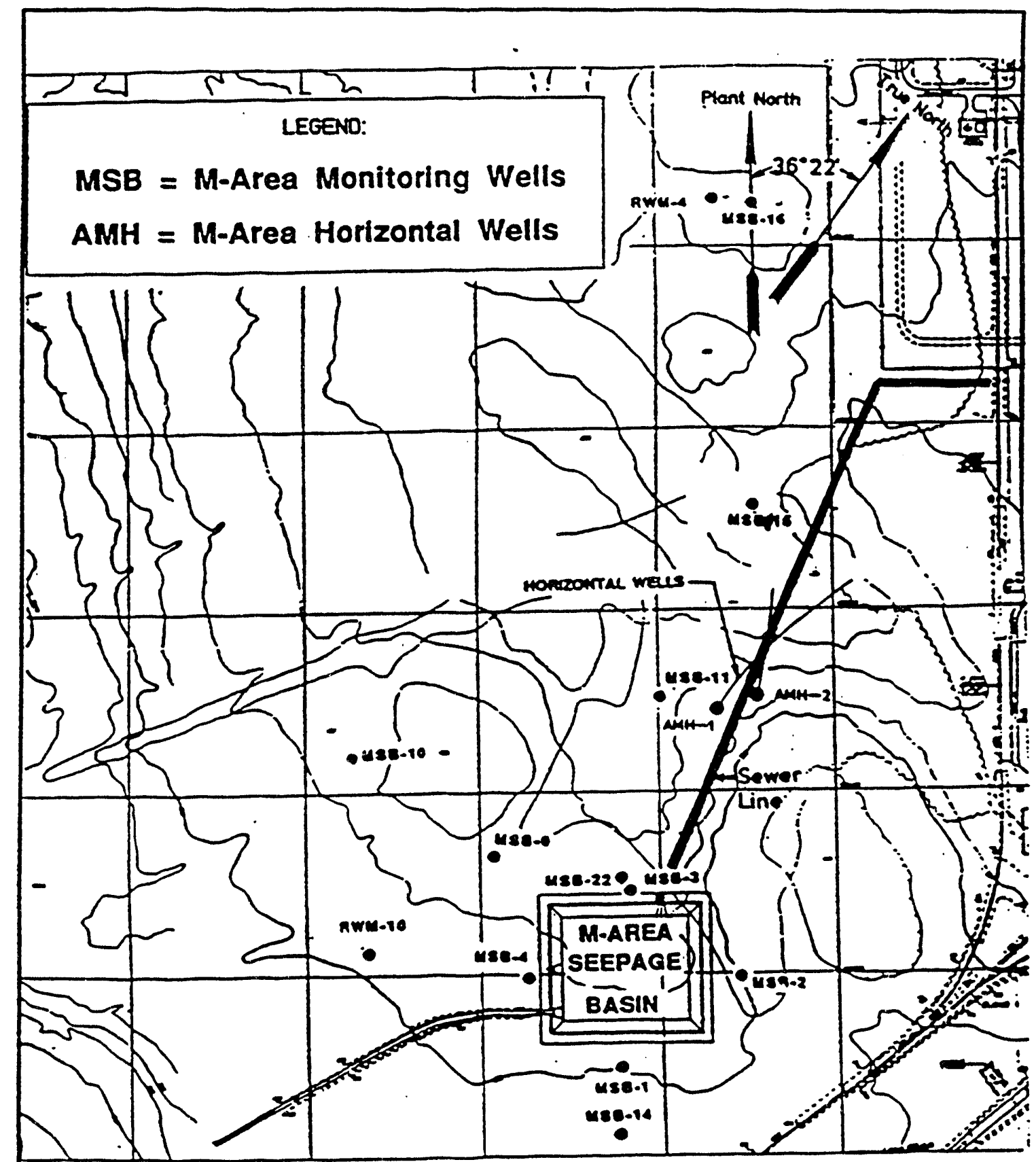

Figure I.2. M-Area and vicinity, Savannah River Plant [from Fig. 2.2, Kaback et al., WSRC-RP-89-784 (1989)] 
Several M-Area remediation project locations are shown on Figure 1.3:

- A pump and treat (P\&T) system, using wells RWM-1 through RWM-11;

- A sediment vapor extraction (SVE) test, using wells VB-1 through VB-3;

- An in-situ air-stripping (ISAS) test, using horizontal wells AMH-1 and AMH-2.

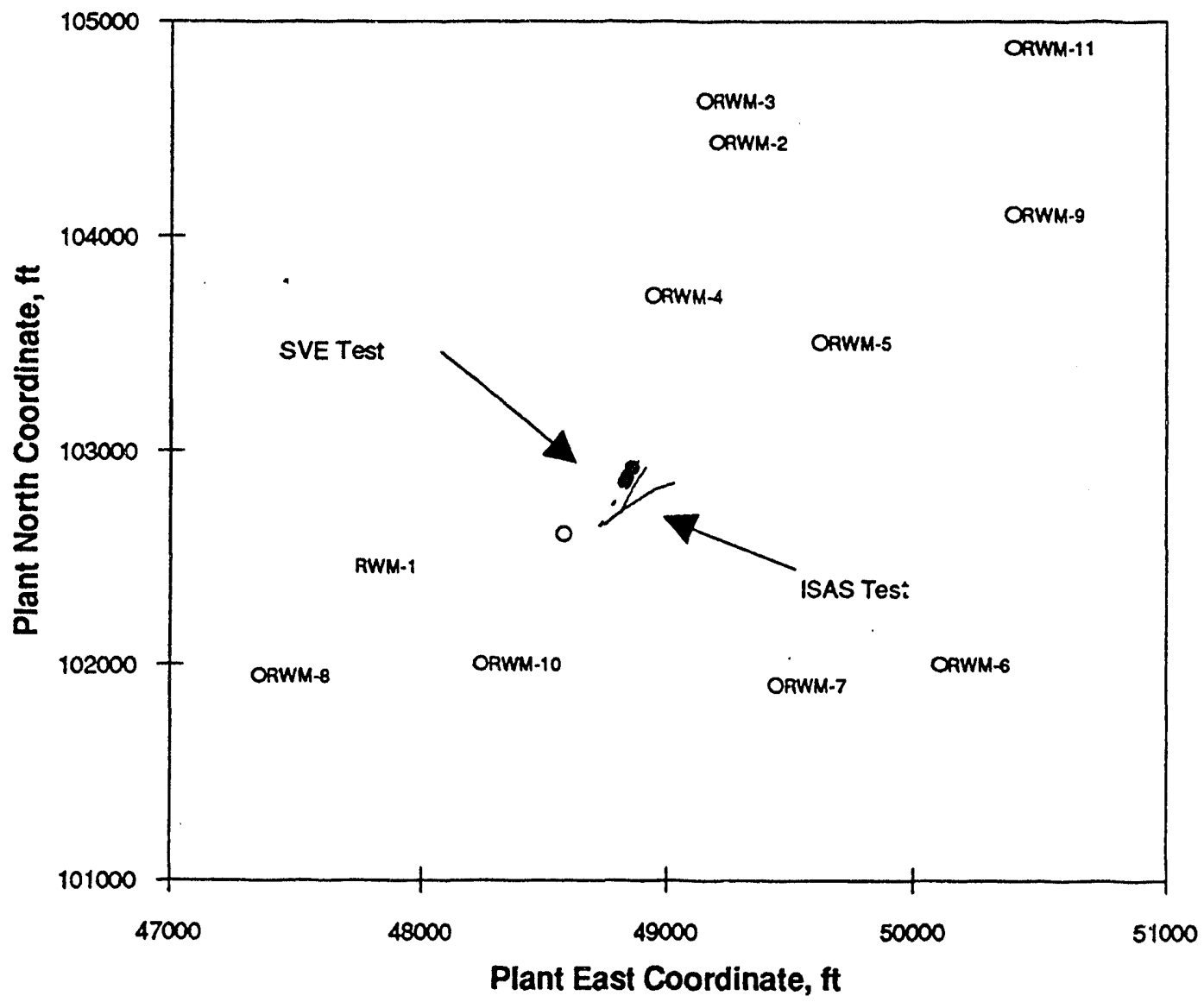

Figure I.3. Remediation wells in M-Area, Savannah River Plant, showing the scale of the Pump and Treat operation in comparison to the test sites.

\section{Full SCale Pump and Treat}

A remedial action program to minimize the migration of contaminated ground water and to remove the contaminants has been in operation since 1985, pumping approximately 380 gallons per minute (gpm.) from 11 wells to a conventional air-stripping tower, where the TCE and PCE concentrations are reduced to less than $1 \mathrm{ppb}$ in the effluent discharged to NPDES outfall A-014 (Colven et al., 1987). The TCE and PCE stripped from the groundwater are discharged to the atmosphere.

According to an oral presentation by Sayannah River personnel in March 1992, more than 10 billion gallons of water had been treated by June 1991 to remove on the order of 250,000 pounds of VOCs, at which time the inlet concentration to the air-stripper was still more than $11,000 \mathrm{ppb}$, about one-third of the average first-year concentration of $32,740 \mathrm{ppb}$. Estimates of the initial inventory of solvents in the area affected by these weils ranged from 266,000 pounds to 449,000 pounds in the report by Colven, et al. (1987). 
Although the concentration of contaminants in the feedwater to the air stripping unit has decreased significantly since 1985 , it is still re' 1 tively high and decreasing very slowly. It appears likely that the initial estimates of contaminant inventory within the aquifer are low, that a considerable additional inventory is contained in the vadose zone and continues to supply contaminants to the aquifer, and that the efficiency of removing contaminants dissolved in water is reduced by the heterogeneity of the geologic media both above and below the water table. Determining the relative contribution of each of these factors to the overall performance is one objective of performing a detailed performance analysis of this remediation technology.

\section{VERTICAL WELL SVE TEST}

A more detailed plan view of the SVE and ISAS test wells is shown on Figure 1.4. The MHT and MHV wells were completed to monitor the aquifer and the vadose zone, respectively, during the ISAS test.

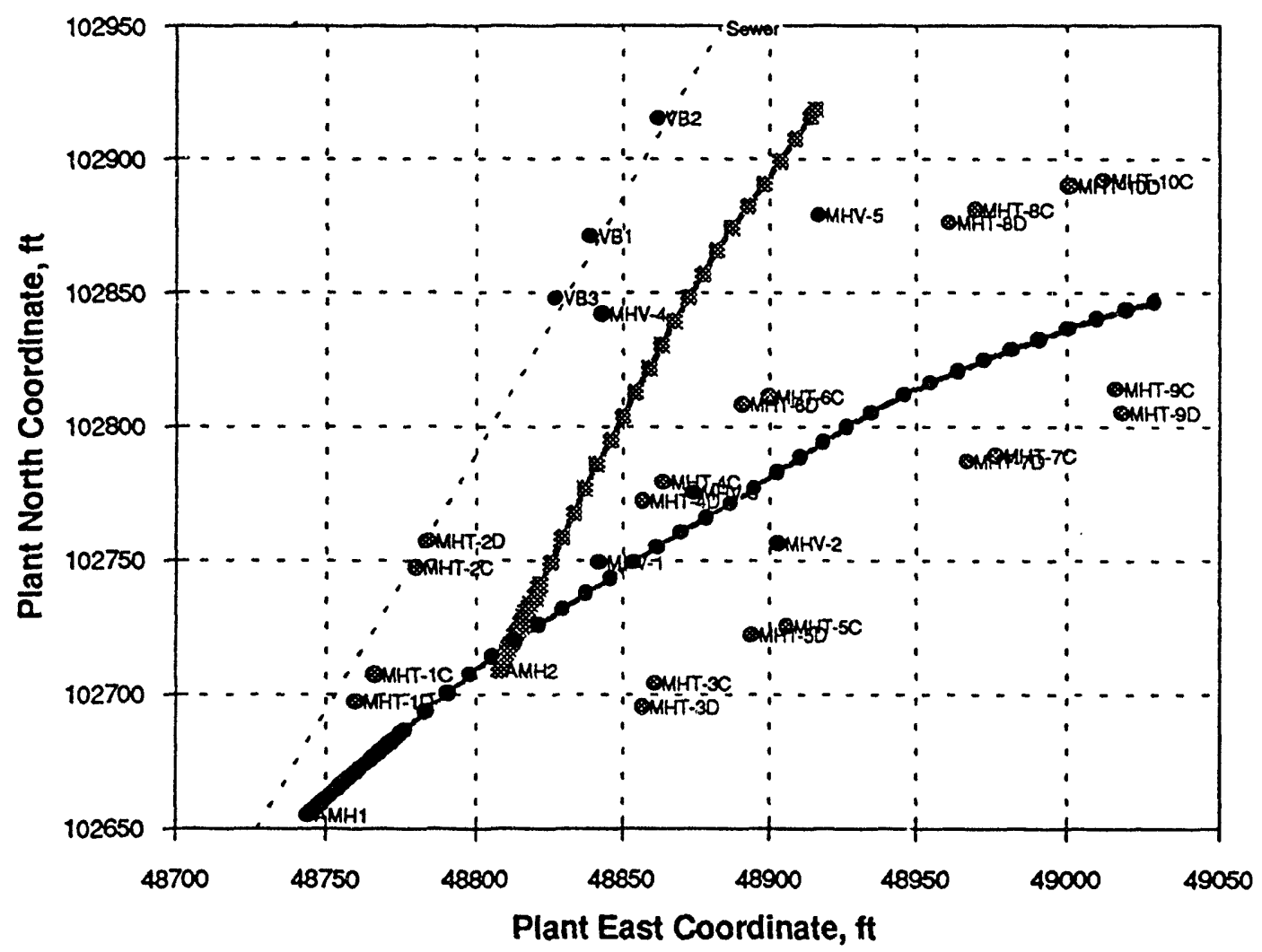

\section{Figure L.4. Detailed plan view of remediation and monitoring wells for the SVE test and the ISAS test, Savannah River Plant.}

One obvious possibility for improving the overall effectiveness of the remediation program is to remove contaminants directly from the vadose zone before they have a chance to reach the water table. The first demonstration at Savannah River of a process of this type was a short-term vacuum-extraction pilot test conducted for a total of 21 days in March, 1987 (Looney et al., 1991a). The three test borings shown in Figure I.4, VB2, VB1, and VB3, were drilled in a straight line running from plant northeast to plant southwest, essentially parallel to and 5 feet to plant northwest of the process sewer line running from MArea operations to the M-Area settiing basin. 
VB1 was completed as the principal extraction well, screened and gravel-packed from a depth of about 24 $\mathrm{ft}$. (elevation $342 \mathrm{ft}$ ) to $124 \mathrm{ft}$. (elevation $242 \mathrm{fL}$ ), about $3 \mathrm{ft}$. below the water table elevation of $245 \mathrm{ft}$.

$\mathrm{VB} 2,50 \mathrm{ft}$. to plant northeast of VB1, was completed in three intervals with separate access tubes: $\mathrm{C}$, from $24 \mathrm{ft}$. to $50 \mathrm{ft}$. (elevation $316 \mathrm{ft}$ to $342 \mathrm{ft}$ ); A, from $58 \mathrm{ft}$ to $92 \mathrm{ft}$. (elevaion $274 \mathrm{ft}$. to $308 \mathrm{ft}$ ); and B, from $116 \mathrm{ft}$. to $137 \mathrm{ft}$. (elevation $229 \mathrm{ft}$. to $250 \mathrm{ft}$.). The water table elevation reported at this location was 239 feet, about 6 feet lower than in VB1.

VB3, $25 \mathrm{ft}$. to plant southwest of VB1, actually comprises two borings. The first one, VB3-C, is a single completion gravel-packed from $19 \mathrm{ft}$ to $30 \mathrm{ft}$. (elevation $336 \mathrm{ft}$ to $347 \mathrm{ft}$ ). The second boring is completed as VB3-A, from $104 \mathrm{ft}$. to $125 \mathrm{ft}$. (elevation $241 \mathrm{ft}$. to $262 \mathrm{ft}$ ), and as VB3-B, from $48 \mathrm{ft}$. to 80 $\mathrm{ft}$. (elevation $286 \mathrm{ft}$. to $318 \mathrm{ft}$.). The reported water table elevation at this location was $243 \mathrm{ft}$.

The test sequence was rather complex, with five of the seven separately-screened "wells" connected sequentially to vacuum for about one day each, followed by a day of simultaneous extraction from all five. Finally, VB1 alone was extracted for the remainder of the test. The total amount of contaminant removed was $1496 \mathrm{lb}$., comprising $1036 \mathrm{lb}$. of TCE and $460 \mathrm{lb}$. of PCE, at air extraction rates of 400 to 500 SCFM (Standard Cubic Feet per Minute). Some additional details are given in Looney et al. (1991a), although the precise test sequence and flow rates are not included. Significant reductions in vapor-phase concentrations (2650 to $147 \mathrm{ppm}$ and 960 to $61 \mathrm{ppm}$ for TCE and PCE, respectively) were observed in the air extracted from VB1 during the test.

\section{HORIZOiNTAL WELL ISAS TEST}

Subsequently, Westinghouse Savannah River Corporation initiated a project to drill and install two horizontal wells, AMH-1 and AMH-2, for further testing of vadose-zone remediation technology (Kaback et al., 1989a and 1989b). Figure I.4 shows a plan view of the trajectories of these two wells, and Figure I.5 gives their projection onto a vertical plane along the "plant east" direction. Figure 1.5 also shows the screened intervals in the monitoring wells, hut their surface elevations are not shown.

The horizontal wells were drilled and completed in September and October, 1988, and were used in the Air-Stripping Phase of the U.S. Department of Energy's Office of Technology Development (OTD) Savannah River Integrated Demonstration Project (SRIDP) for the Removal of VOCs at Non-Arid Sites from July 27, to December 13, 1990. They were also used in the In-Situ Bioremediation Phase, which started in the spring of 1992. The extraction well, AMH-2, has a screened interval of approximately 200 feet, and the injection well, AMH-1, has a screened interval of about 310 feet. (Buscheck and Nitao, 1992) $)^{1}$.

Vacuum extraction at approximately 580 SCFM from AMH-2 started July 27, 1990 and continued, with minor interruptions, until December 13, 1990 (Looney, et al., 1991b). During part of the test period, air was injected at various rates below the water table through AMH-1, to test the possibility of air-stripping VOCs from groundwater in situ while using vacuum extraction to remove contaminants from the vadose zone.

During the first 21 days of operation, a total of approximately $2696 \mathrm{lb}$. of VOCs was removed, compared to about $1496 \mathrm{lb}$. for the vertical well air extraction test in the same amount of time. This period included 15 days of extraction only, followed by 6 days of extraction combined with injection into AMH-1 at 65 SCFM. Hence, the horizontal-well extraction, combined with 6 days of horizontal-well air injection, removed about $80 \%$ more VOC mass than a vertical-well system operated for the same length of time.

\footnotetext{
${ }^{1}$ The 310-foot length of perforated wabing in AMH-1 given by Buscheck and Nitao agrees with the value on page 8 of Kaback a. al. (1989). However, there is no value given for the screen length of AMH-2 in this reference. On page 9, in is stated that wire-wrapped screen was installed to a depth of 232 feet, with a five-foot section of flexible drill pipe attached at the leading edge of the screen. Figure 5 , however, indicates a cave-in at 205 feet. Although this dra wing is not to scale, dimensions on the figure indicate that the lop of the screen is about 15' from the surface (in the vertical section of the boretiole) and that the distance along the borchole from the surface to the 'isginning of the "straight, horizonial" section is about 103.5. Assuming that the end of the 5 section of flexible drill pipe is at the cave-in (205"), the length of screen would be $205^{\circ}-5^{\prime}-15=185$, instead of the value 200 given by Buscheck and Nitao (1992).
} 


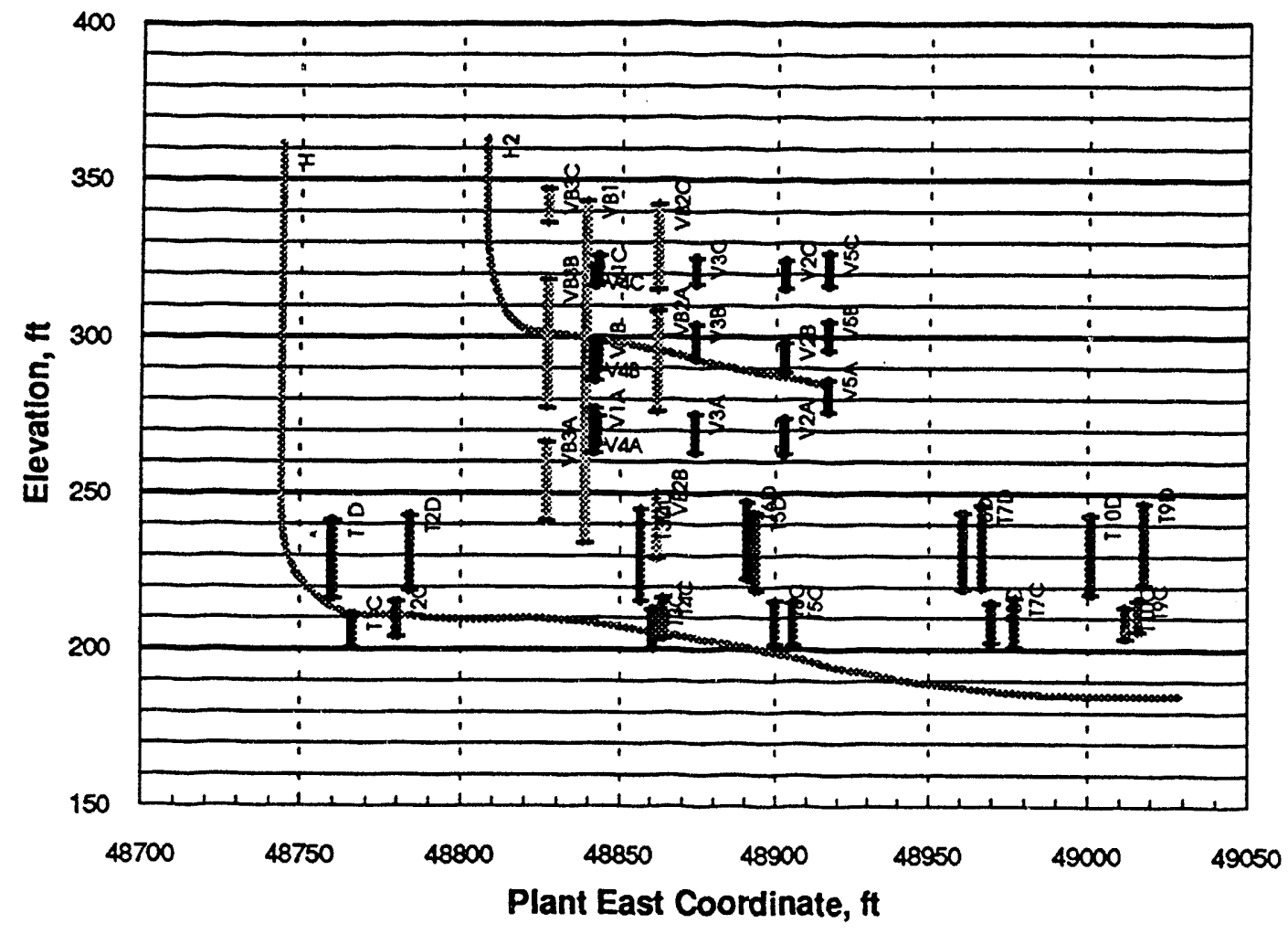

Figure I.5 Projection of trajectories of horizontal boreholes AMH-1 and AMH-2, and monitoring well screens, on a vertical plane oriented along the plant east-west direction.

During the air-stripping test period of 139 days elapsed time, total removal of VOCs was about $15923 \mathrm{lb}$. It should be noted, however, that the pump and treat operation removed about $5342 \mathrm{lb}$. of VOCs during its first 615 hours ( 21.5 days) of operation, and about $23,200 \mathrm{lb}$. during its first 133 days.

\section{PERFORMANCE COMPARISONS}

Since the remediation operations discussed above have not been completed (i.e., continued to the point that remaining concentrations are in compliance with groundwater standards or until essentially complete removal of the initial contaminant inventory has been effected), comparison of their performance is necessarily imprecise. Perhaps it is appropriate to list some of the reasons that the performance would be expected to differ:

1. Different carrier fluids transport contaminants from the sediments to the extraction wells: water for the pump and treat process; air for both the vacuum extraction and air stripping operations.

2. Different operating protocols were used: carrier fluid extraction alone for the pump and treat and vacuum extraction processes; both extraction and injection of carrier fluid for the air stripping test.

3. Different well configurations were used: vertical wells for the pump and treat and vacuum extraction processes; horizontal wells for the air stripping process.

4. Qualitatively different subsurface regions are swept by different carrier fluids: saturated zone only for the pump and treat process, vadose zone only for the vacuum extraction process, and both zones for the air stripping process. 
5. Different volumes of sediments, at variable and unknown distances from the original sources of VOC infiltration, are affected by the three processes - i.e., the spatial and temporal scales are quite different, with the pump and treat operation covering a much larger area than the other two. Figure I.3 shows that the size of the experimental area is much smaller than the average interwell distance in the pump and treat array. Presumably, the larger area would have a more diffuse contaminant plume than the small "hot spot" near the sewer line.

6. Finally, because the interbedding of sands and clays forms an extremely heterogeneous geologic system, both the initial contaminant distribution and the flow and transport paths during remediation are highly variable, causing large expected differences from place to place even if all controllable variables were to be kept the same.

Since air is the carrier fluid for both the vacuum extraction and air stripping tests, we can compare these two tests more or less directly. However, the air extraction rates were different: 400 to 500 SCFM for the former, and 580 SCFM for the latter. Using 450 SCFM as an average for the SVE test, the VOC removed in 21 days was $1496 / 450=3.32 \mathrm{lb}$. per SCFM. For the ISAS test, the comparable quantity is $2696 / 580=$ $4.65 \mathrm{lb}$. per SCFM. On this basis, the improvement of air-stripping compared to vacuum extraction is $100 *(4.65-3.32) / 3.32=40.1 \%$. How much of this improvement is due to the well configuration, how much to the injection of air, and how much to the different locations and sediment volumes affected, remains to be determined.

This normalization partially corrects for differences in one of the major operating costs - i.e., the cost of handling large volumes of air. However, since no adjustment was made for the fact that ISAS requires injection as well as extraction of air, this comparison tends to overstate the improvement of ISAS relative to SVE. If the injection rate is simply added to the extraction rate for the last 6 days, the average air rate is almost 600 SCFM, and the average extraction rate drops to $4.50 \mathrm{lb}$. per SCFM. This represents an improvement of $35.7 \%$, an insignificant change from the estimate based on the extraction rate alone. However, for the later stages of the test, when higher rates and pressures were used during the injection, the adjustment would be larger.

Another way of comparing results is to normalize them for the total length of the screened intervals active during the operation. While the air-rate normalization to some extent adjusts for operating costs, the use of active screen length would tend to adjust for differences in capital costs. This is somewhat analogous to the concept of the productivity index for oil wells used in the petroleum literature or the concept of specific discharge for water wells. Since the cost of drilling is a major part of the capital required to implement a remediation project, a measure based upon the mass removed per foot of active wellbore would give a rough indication of the relative effectiveness of different options.

Table 2.1 in Colven et al. (1987) gives the screened intervals for each of the 11 wells used in the pump and treat system. All wells except RWM-1 each have four ten-foot screens; RWM-1 has one 60' screen. Hence a total of $460^{\prime}$ of screened interval is active throughout the pump and treat operation.

For the vertical well SVE test, Table I.1 for the screened intervals was prepared from the well logs given in Appendix B of Looney et al. (1991a). As noted above, the SVE test sequence was rather complex and cannot be reconstructed completely from data given in Looney et al. (1991a). The data given by Looney in Appendix $\mathrm{D}$ were used to construct an approximate activity sequence for the first 5.8 days of operation. This sequence is summarized in Table 1.2. 
Table I.1. Screened interval data for wells used in the Savannah River Sediment Vapor Extraction (SVE) test.

\begin{tabular}{|c|c|c|}
\hline Screen Label & Screened Depth Interval, $\mathrm{ft}$ & Active Wellbore, $\mathrm{ft}$ \\
\hline VB1 & 24 to 124 & 100 \\
\hline VB2C & 28 to 48 & 20 \\
\hline VB2A & 60 to 90 & 30 \\
\hline VB2B & 117 to 137 & 20 \\
\hline VB3C & 20 to 30 & 10 \\
\hline VB3B & 50 to 80 & 30 \\
\hline VB3A & 105 to 125 & 20 \\
\hline
\end{tabular}

Table I.2. Approximate sequence of active screens during the short term SVE Test at Savannah River.

\begin{tabular}{|c|c|c|}
\hline Time, $\mathbf{h r}$ & Screens on Vacuum & Total Active Feet of Screen \\
\hline 21 & VB1 & 100 \\
\hline 7 & VB3B & 30 \\
\hline 15 & VB3B,VB1 & 130 \\
\hline 25 & VB3A & 20 \\
\hline 24 & VB2A & 30 \\
\hline 24 & VB2C & 20 \\
\hline 22 & VB1,2A,2C,3A,3B & 200 \\
\hline
\end{tabular}

Page 6 of Looney et al. (1991a) states that "by the end of the test, 15 days of vacuum extraction from VB1 had been performed." Elsewhere, it is mentioned that the entire test lasted for 21 days, and the total time for the sequence given in the table above is 5.8 days (138 hours). We will assume, for the purposes of normalizing the results on the number of feet of active wellbore, that, for the remaining time of $\mathbf{1 5 . 2}$ days ( $365 \mathrm{hr}$ ), VB1 was operated on vacuum and that the 15 days mentioned above did not include the extraction from VB1 during the early stages of the test. Then the time-average length of screen active during the SVE test is $(21 * 100+7 * 30+15 * 130+25 * 20+24 * 30+24 * 20+22 * 200+365 * 100) /(24 * 21)=93.0$ ft.

Buscheck and Nitao (1992) noted that screen lengths in AMH-1 and AMH-2 are $94.5 \mathrm{~m}$ and $61.0 \mathrm{~m}$, respectively, or approximately $310 \mathrm{ft}$ and $200 \mathrm{ft}$. Appendix E of Kaback et al. (1989b) contains screen and casing records for these wells prepared by Sirrine Environmental Consultants, resulting in screen lengths of $309.7 \mathrm{ft}$ for $\mathrm{AMH}-1$ and $204.7 \mathrm{ft}$ for AMH-2. These were used as the active screen lengths in the calculation of the average active length, as follows:

1. There was no air injection for the first 16 days of the ISAS test. Hence, for the first 21 days, the average active length was $(21 * 204.7+5 * 309.7) / 21=278.4 \mathrm{ft}$. 
2. For the entire 139 days of elapsed time during the ISAS test, there was a total of 120 days of actual operation, based upon 2879.1 hours of operating time given in Excel spreadsheet files supplied by B. B. Looney in May 1992. The extraction well alone was active for about 40 days, and both wells were used for about 80 days of actual operating time (i.e., with down time subtracted). Then the average active length over the life of the test is $(80 * 309.7+120 * 204.7) / 120=411.2 \mathrm{ft}$.

Table I.3 summarizes the performance of the three remediation processes tested at Savannah River for comparable time periods. The P\&T data were taken from Colven et al. (1987), interpolating where necessary, and the ISAS data were taken from the Excel spreadsheets supplied by B. B. Looney in May 1992.

Table I.3. Comparison of performance of remediation processes tested at Savannah River, normalized for different well screen lengths.

\begin{tabular}{|lcccc|}
\hline PROCESS & DAYS & POUNDS REMOVEd & FT OF SCREEN & POUNDS PER FOOT \\
SVE & 20.8 & 1496 & 93.0 & 16.1 \\
P\&T & 21.5 & 5342 & 460.0 & 11.6 \\
ISAS & 20.2 & 2696 & 278.4 & 9.7 \\
ISAS & 20.9 & 2767 & 278.4 & 9.9 \\
P\&T & 120 & 21034 & 460.0 & 45.7 \\
ISAS & 120 & 15923 & 411.2 & 38.7 \\
\hline
\end{tabular}

As can be seen readily from Table I.3, performance of the pump and treat process is essentially the same as the horizontal-well air stripping process when normalized on the basis of the number of feet of wellscreen active during the operation, for both short and long time periods. In fact, the normalized performance for ISAS is only about $85 \%$ of the P\&T performance at both 21 days and 120 days of operating time. In other words, the ratio of cumulative contaminant removal for these two processes does not vary with operating time for periods up to 120 days. This suggests that long-term comparisons of relative rates of removal could possibly be based on shorter-term tests.

The vertical-well SVE normalized test performance for three weeks of operation is higher than the other two processes, but this may be an artifact of having several one-day extraction tests from different screened intervals. Since the concentration of VOCs in any carrier fluid generally decreases very rapidly at the start of an extraction operation, it might be expected that the combined effect of six one-day tests would remove more contaminant than a single six-day extraction. It is unfortunate that SVE was not continued long enough for this effect to dissipate.

Clearly, more thorough and sophisticated analyses are required in order to determine which of these remediation technologies is most effective, and to understand how to design and implement their use in different geologic and climatic settings. Additional field experience is also needed.

The remaining sections of this report document the use of a number of different approaches to developing reliable, practical performance analysis methods for evaluating technologies to remediate sediments and aquifers contaminated with VOCs, using the Savannah River data to provide test cases. 


\section{Remediation PerformanCe}

\section{IDEAL SYSTEM}

Consider a tank full of contaminated water, with no source of water inflow. Removing the contaminant from this ideal system is simply a matter of pumping out its total volume, and the cumulative fraction of contaminant removed is directly proportional to the cumulative fraction of water removed. A plot of contaminant concentration $v s$. time would remain constant at its initial value until all the contaminant had been removed, then drop to zero.

\section{REAL SYSTEMS}

Now suppose that we allow uncontaminated water to enter the tank while we are pumping, and that the pathways for water flow through the tank are highly variable, so that some pathways are quickly swept clear of contaminant by the inflow of clean water, while others take much longer. At any time (or, equivalently for constant extraction rate, cumulative water withdrawal), the water being pumped out will be a mixture of clean and contaminated water. The rate of contaminant removal will then gradually decrease with time, since it will depend upon the proportions of clean and contaminated pathways contributing to total outflow.

The variability in flow pathways arises from several sources, including the spatial distribution of sources and sinks (flow geometry), instabilities in the physical processes goveming the displacement of one fluid by another, and the heterogeneity of the porous media within which the displacement occurs. Each source affects the design and operation of remediation projects differently, and will be considered in later sections of this report.

\section{Flow Geometry}

In a completely uniform porous medium, with a given distribution of sources and sinks, the steady-state flow of incompressible fluids can be analyzed by potential theory, and the flow regime is completely described by a family of constant pressure contours and streamlines. The streamlines can be regarded as the trajectories of fluid particles as they travel from sources to sinks. Since the travel time will vary depending upon the path followed, the fluid reaching the outflow boundaries (sinks) will generally be a mixture of clean and contaminated carrier fluid.

These effects are illustrated for several choices of flow geometry in Section III and Appendix A.

\section{Instabilities}

Instabilities in fluid displacements arise from density differences, as in the downward displacement of a less dense by a more dense fluid, from viscosity differences, when a less viscous fluid displaces a more viscous one, and from other causes. These phenomena are considered in more detail in Appendix D.

The principal effects of instabilities at Savannah River probably occur during the infiltration of the contaminants through the vadose zone, causing at least some of the observed spatial variation in initial contaminant concentrations, and consequently contributing to some of the variation in performance of the remediation processes. The injection of air below the water table also creates an unstable displacement.

\section{Heterogeneity}

All geologic materials are heterogeneous, to some degree, at any scale of measurement, with physical and chemical properties changing either gradually or abrupuly from point to point. Most models of flow and transport processes are based conceptually upon continua, and their degree of success in predicting the behavior of real systems depends upon how well the actual spatial variability of physical properties is 
approximated in the model, and how completely the physical and chemical processes important to process performance are represented.

The degree of variability and its spatial scale depend upon the specific property being estimated. For example, the permeability of geologic media can easily vary over five or six orders of magnitude even within distances of a few tens of feet or less, but the fractional porosity is physically constrained to lie between zero and unity. In turn, these different properties have varying impact on the performance of in situ remediation projects and others, such as oil and gas recovery, which also depend upon the flow and transport of substances in natural media.

\section{Geologic Origin}

Porosity, permeability, mineralogy, and other properties of geologic strata depend upon the specific processes responsible for their formation or deposition and the uniformity of conditions affecting these processes. For example, sediments deposited by rivers vary enormously depending upon whether they are from channels, point bars, or other identifiable characteristic depositional features common to all rivers. These variations are attributed to the differences in energy of the sediment transport medium (water), with higher, relatively constant energy (flow velocity) favoring the deposition of well-sorted, more uniform sediments and lower, variable energy leading to less uniform deposits. Within a particular depositional sub-environment of a river complex, such as a point bar, flow properties may be highly variable and stratified in the vertical direction but reasonably uniform laterally.

Other depositional environments, such as shallow marine complexes (deltas, barrier islands, lagoons, etc.) and dune fields (wind-blown, or eolian, deposits) have their own characteristic distributions of properties important to flow and transport. To further complicate matters, diagenetic processes (i.e., those affecting the material after its initial deposition or formation) such as compaction, dewatering, solution, precipitation, cementation, etc. can introduce even more heterogeneity than the material possessed initially.

Over the last two or three decades, enormous progress has been made within the petroleum industry in understanding genetic and diagenetic processes affecting sedimentary rocks (both clastics and carbonates), and, more importantly for the analysis of remediation processes, in understanding how to use this information quantitatively to model more realistically the flow of fluids and the transport of dissolved substances in the subsurface. A particularly good summary of this approach and the data and analysis requirements is given by Weber and van Geuns (1990).

\section{Contaminant Distribution and Initial Inventory}

Geologic heterogeneity affects the initial distribution of contaminants as well as the flow patterns of water and air during the operation of a remediation project. The measured concentrations of contaminants in samples (carefully taken to avoid contamination and mixing) of both sediment and water commonly vary by several orders of magnitude within a single borehole, as well as from borehole to borehole within correlative strata. In sediment samples from the vadose zone at the SRIDP site, reported TCE concentrations varied from less than the detection limit of $2 \mathrm{ppb}$ to more than $16,000 \mathrm{ppb}$, and PCE concentrations varied from the same lower limit to almost $9,000 \mathrm{ppb}$ (Eddy et al., 1991), a range of almost four orders of magnitude.

The measured concentrations themselves are subject to large errors, not because of limited analytical precision or heterogeneity per se but because the process of sampling subsurface sediments and bringing them to the surface for analysis can lead to large changes in concentration compared to undisturbed conditions. An example was given (for samples taken below the water table) by Bishop et al. (1990) at the Lawrence Livermore National Laboratory site, where PCE has contaminated sediments originally deposited in a fluvial environment. Flushing by mud filtrate during coring appears to have removed $60 \%$ to $75 \%$ of the original water content of the sediment, with a corresponding reduction in PCE concentration in the sample pore water, based upon differences in calcium and sodium ion concentrations between water centrifuged from the samples and discrete groundwater samples. 
At Savannah River, flushing was not a problem in the vadose zone because the samples were obtained as drive cones using a split spoon sampler. However, there will almost certainly be a significant loss of contaminants from the vapor. phase. In situ, the total mass of VOCs in the vapor phase is of the same magnitude as in the aqueous phase; hence, the reported contaminant concentrations may be low. Losses from either vadose zone or aquifer samples are most likely to be greatest in the most permeable sediments, which may at least partially account for the observation that fine-grained sediments tend to have more contaminant than coarse-grained sediments. Thus, heterogeneity contributes at least indirectly to the difficulty in estimating the amount of contaminant initially present and to the problems of comparing concentrations in extracted air or water to concentrations in situ.

At typical vertical spacing of one or two meters between sample points, adjacent sample concentrations can differ by factors of 10,100 , or more, and the same data could support very different estimates for the total contaminant inventory depending upon the method used for interpolating between measured values. Although some of this observed heterogeneity arises from such causes as a spatially and temporally nonuniform distribution of contaminant sources or localized variations in infiltration and runoff, the dominant effects in most cases results from the intrinsic heterogeneity of the geologic media. The resulting uncertainty in the initial contaminant inventory can be reduced by more closely-spaced sampling. If we are willing to spend enough money, the errors in parameters simply related to measurements can be reduced arbitrarily close to zero.

\section{Extraction Efficiency}

There are, however, consequences of heterogeneity for contaminant transport which cannot be removed by gathering more data. To illustrate this point, refer to Fig. II.1.

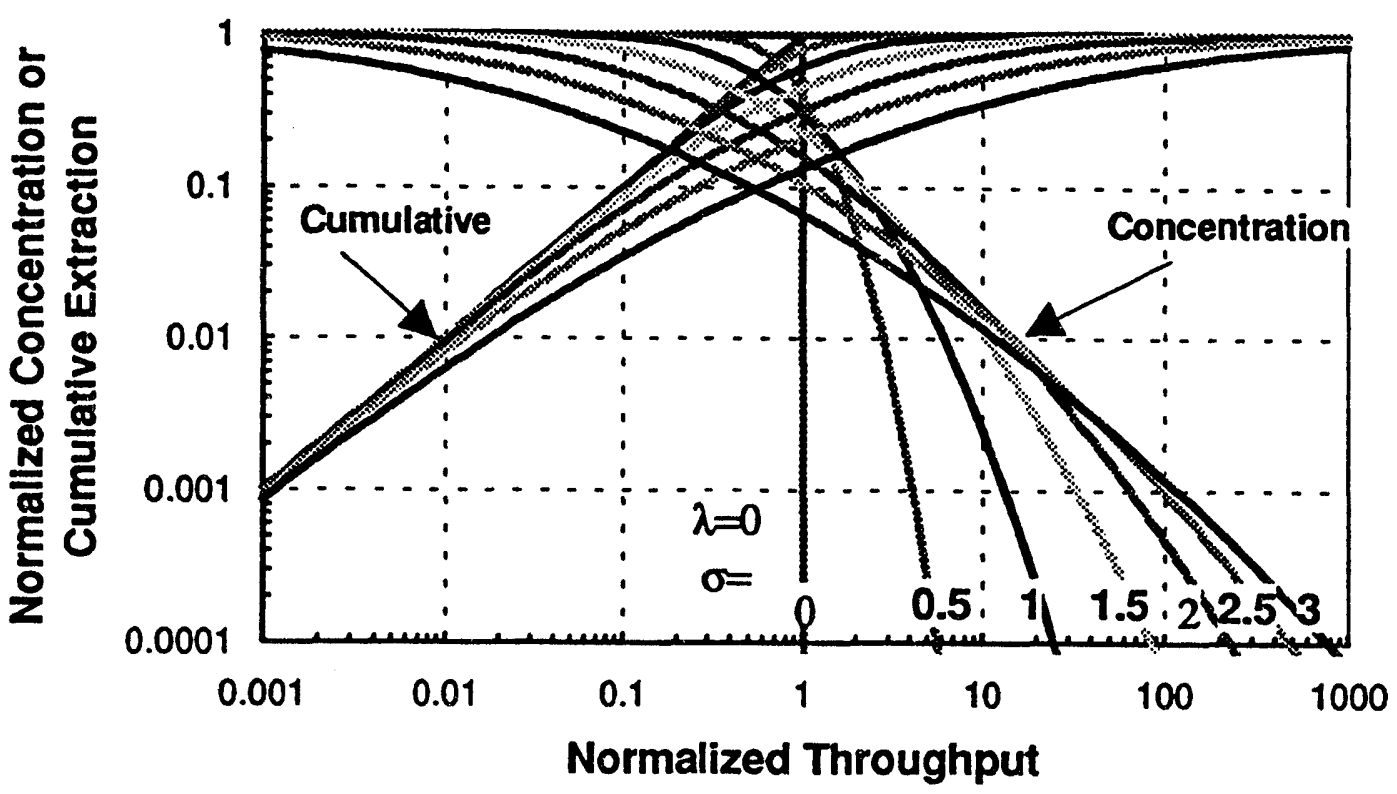

Figure II.1. Normalized performance curves for contaminant extraction from heterogeneous media. The heterogeneity parameter, $\sigma$, is zero for an ideal homogeneous system. The larger the value of $\sigma$, the greater the degree of heterogeneity and the longer a remediation project will have to operate to achieve given remediation targets.

This figure is based (see Section IV and Appendix B) on an extension of a simple model described by Chesnut, Cox, and Lasaki (1978) for waterflooding petroleum reservoirs. A similar model for 
groundwater travel time associated with nuclear waste repositories was presented recently by Chesnut (1992).

In these models, flow pathways are assumed to be distributed between inflow and outflow boundaries according to a log-normal probability density function with standard deviation $\sigma$ (basis: natural logarithms). For $\sigma=0$, the plot of cumulative contaminant extracted vs. throughput is exactly like it would be for pumping contaminated water from a tank as described above, and the corresponding normalized concentration remains constant until exactly one tank volume has been pumped out (i.e., the normalized throughput reaches 1.0 ), then drops to zero. This represents the ideal case of stable linear ("piston-like") fluid displacement in a perfectly homogeneous medium. There is no mixing of contaminated with uncontaminated fluid.

The normalized concentration vs. normalized throughput curves are labeled with the selected values of $\sigma$ : $0.0,0.5,1.0,1.5,2.0,2.5$, and 3.0. These labels are omitted from the corresponding normalized cumulative extraction curves to avoid clutter, but they should be easy to distinguish if one keeps in mind that larger values of $\sigma$ require larger amounts of throughput to reach a given fraction of contaminant removal - e.g., a throughput of 0.99 is required to remove $99 \%$ of the contaminant for $\sigma=0$, but a throughput of more than 2.5 would be required to reach the same percentage removal for $\sigma=0.5$.

As $\sigma$ increases, the displacement becomes less and less "piston-like," and the throughput required to achieve a desired reduction in contaminant concentration increases many-fold. For example, typical contaminant concentration targets for remediation are on the order of 5 or $10 \mathrm{ppb}$. For groundwater concentrations of more than $10,000 \mathrm{ppb}$ found at Savannah River, the required reduction is more than 3 orders of magnitude. Reducing the contaminant concentration by a factor of 1000 requires between 4 and 5 times the ideal throughput for $\sigma=0.5$, and more than 10 times the ideal throughput for $\sigma=1.0$. A value of 0.5 represents a degree of homogeneity seldom seen in field-scale displacement processes, and many commercially successful waterflood projects exhibit performance consistent with $\sigma$ equal to 0.8 or higher.

Although the parameter $\sigma$ is introduced in the derivation of these models as the standard deviation of an assumed log-normal distribution of permeability, it seems, in practice, to account, at least to first order, for all effects causing fluid displacement to depart from the "piston-like" ideal, including viscous or gravitational instabilities and the distribution of streamlines resulting from non-linear flow paths. The functional forms relating concentration vs. throughput and cumulative removal vs. throughput seem capable, in most cases, of adequately representing both field data and the results of much more complex finite-difference and similar models. However, the numerical value of $\sigma$ often must be adjusted from the value derived from permeability data alone, especially when time-dependent processes (such as diffusion or imbibition) are significant.

In this highly simplified model, only four parameters are used to determine the rate and cumulative amount of contaminant removed as functions of time or cumulative carrier fluid extracted:

- The total initial inventory of contaminant.

- The average initial concentration of contaminant in the carrier fluid within the bulk capture volume.

- The fraction of the bulk capture volume which is contaminated.

- The heterogeneity parameter.

The actual application of this model depends upon developing methods of determining these parameters for specific remediation processes, geologic settings, well pattems and types, extraction and/or injection rates, etc., from characterization and other data, and from the use of more complex models to investigate the rôle of specific physical or chemical processes and mechanisms. Provided that it can be properly validated and calibrated in specific geologic environments, it can form the basis for Monte Carlo or other probabilistic analyses of process performance. 
Note that no amount of characterization data will eliminate the "smearing" of contaminant removal as a function of cumulative throughput - it can only tell us how bad the situation is. We can reduce the uncertainty in our predictions of the time and cost required for cleaning up the contaminant, but there is a very. real possibility that in extremely heterogeneous media the cost will be unacceptable. An ultimate goal of a modeling system or strategy is to provide solid, defensible forecasts of remediation performance so that intelligent decisions can be made concerning the allocation of scarce resources, including making sound arguments to regulatory agencies and the public that costs may not be commensurate with benefits.

\section{OBJECTIVES OF ANALYSIS \& EVALUATION}

The fundamental purpose of the Analysis and Evaluation Task is to understand the factors responsible for the observed performance differences summarized in Section I. It is especially important to determine how much of the difference between one test and another is due to the effects of different operating conditions (such as choice of the number, location, and geometry of wells, the choice of a remediation process, etc.) and how much is due to variation in the contaminant distribution and flow paths imposed by the heterogeneities of the geologic media involved.

Freeze and his colleagues, in a four-part series of papers [Freeze et al. (1990), Massman et al. (1991), Sperling et al. (1991), and Freeze et al. (1992)], refer to the different operating conditions that may be chosen as decision variables, since a manager must decide which of the various alternatives is "best" according to some rational and defensible criterion. Without a quantitative understanding of the degree to which these choices affect the performance of remediation processes, it is impossible to optimize these projects, to predict accurately how long might be required, or even to justify the choice of one technology over another. This report documents the progress we have made as of $\$$ sptember, 1992 in developing this understanding, and outlines the direction of future work needed to pursue the promising leads that have been developed up to this point. 


\section{EFFECTS OF FLOW GEOMETRY}

As indicated in Section II, fluid flow between sources (injection wells) and sinks (extraction wells), even in a homogeneous porous medium, is heterogeneous in the sense that streamlines have different lengths, and hence correspond to different travel times for sweeping contaminants to an extraction well. Even if the contaminant is initially uniformly distributed, the extraction rate versus time will depart from that of the ideal system described in Section II and illustrated for $\sigma=0$ in Fig. II.1.

\section{STREAMLDJES}

Figure III.1 shows one quadrant of a streamline contour map for an isolated five-spot well pattern, in which a single injector penetrating an infinite horizontal permeable stratum is located in the center of a square defined by four extractors completed in the same stratum and located at its corners. Multiple replications of this pattern are frequently used in waterflooding petroleum reservoirs. By definition, the total volumetric flow rate between any pair of adjacent streamlines is the same as the rate between any other pair. Hence, the streamline pair, (i.e., stream channel), enclosing the smallest area will have the shortest travel time between injector and producer. This "principal stream channel" is easily seen in

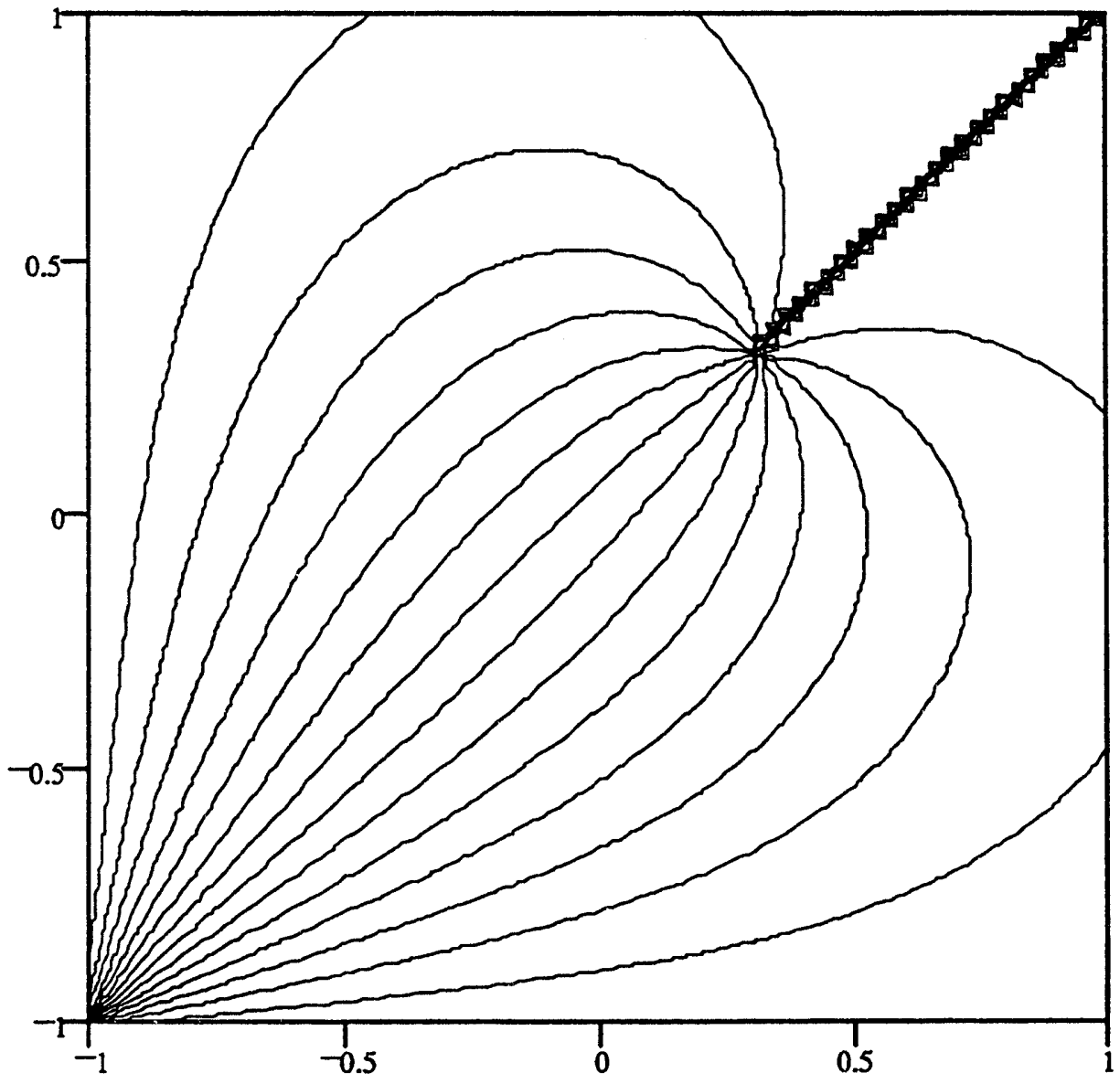

$\Psi$

Figure III.1. Contour map of streamlines in an isolated five-spot pattern. The injection well is located at the bottom left-hand corner and one of the four extraction wells is located near the upper right-hand corner. 
Fig. III.1 to be the channel bracketing the main diagonal directly connecting the injection and production well.

Brigham and Abbaszadeh-Dehghani (1987), in an article on the use of tracer testing for characterizing petroleum reservoirs, presented a clear discussion of the different mechanisms responsible for the mixing of displacing and displaced fluids, including the effects of flow geometry. Theoretically, stream channels are completely independent of each other, and mixing occurs only at the production well, not within the reservoir. They found that the breakthrough of injected fluid at the production well for all common waterflood patterns, including the five-spot, is described by the following equation:

$$
\begin{aligned}
& f_{d}=1-0.5 \cdot\left\{\exp \left[-1.810 \cdot V_{p D}^{0.530}\right]+\exp \left[-0.715 \cdot V_{p D}^{0.972}\right]\right\}, \\
& \text { where } V_{p D}=\frac{V_{p}-V_{p B T}}{1-V_{p B T}} .
\end{aligned}
$$

In Eq. (III.1), $f_{d}$ is the fraction of injected fluid in the produced fluid, $V_{p}$ is the number of pattern pore volumes injected, and $V_{p B T}$ is the number of pattern pore volumes injected at breakthrough of the injected fluid (approximately equal to 0.42 for a five-spot pattern). If the pore volume is assumed to be filled initially with contaminated groundwater at constant contaminant concentration, then $1-f_{d}$ will be the normalized contaminant concentration in water produced from the extraction well, as shown in Fig. III.2.

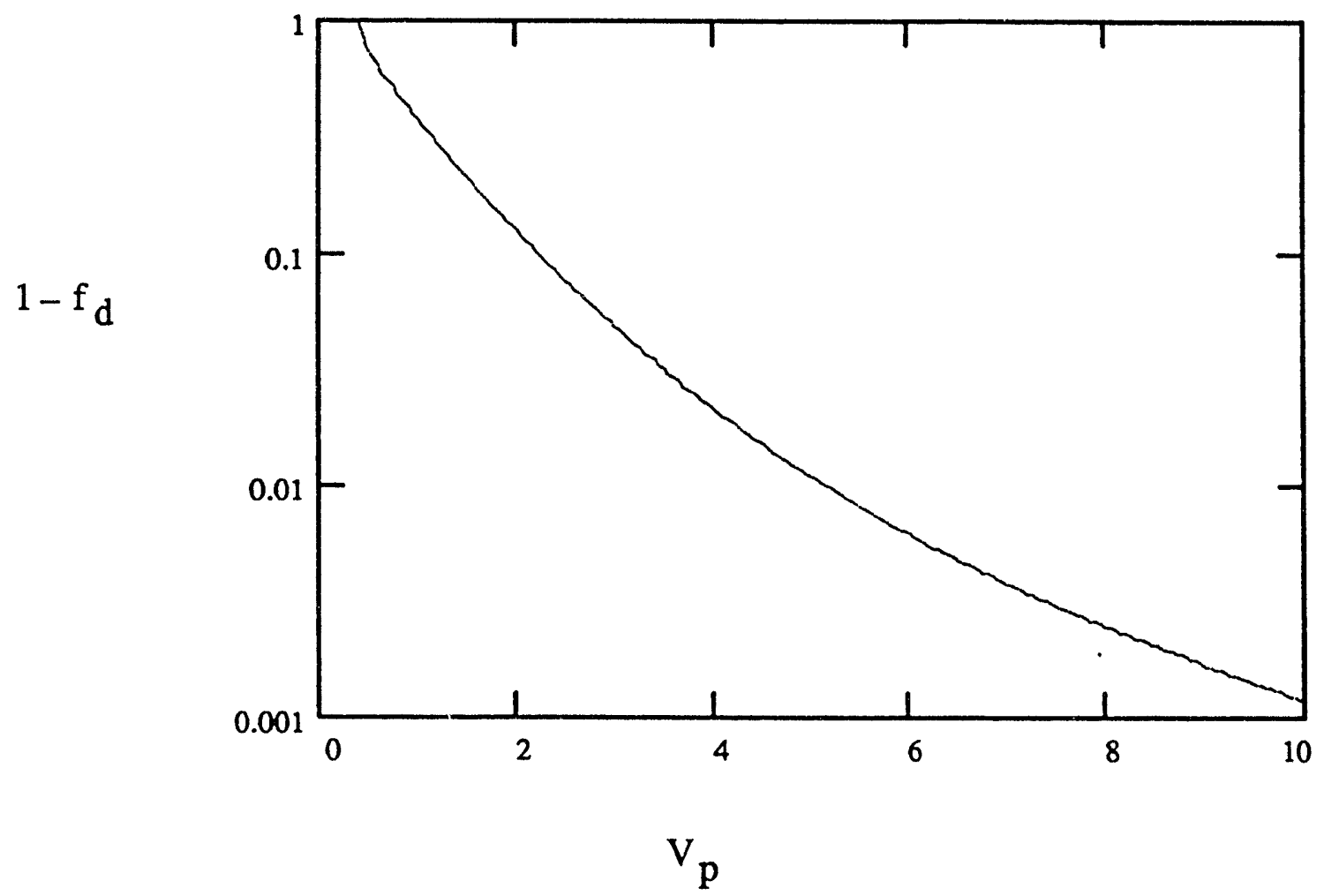

Figure III.2. Normalized contaminant concentration vs. pore volumes of water extracted (or injected) for an ideal homogeneous five-spot initially filled with water at a constant contaminant concentration. 
Note that, in an ideal contaminant removal process, the normalized contaminant concentration in the extracted water would remain equal to unity until exactly one pore volume had been extracted, and would then drop to zero. The "heterogeneity" arising from flow geometry effects causis a pronounced right hand tail to the performance curve, so that about 10 pore volumes would have to be extracted in order to reduce the contaminant concentration to 0.001 of its initial value. We emphasize that this effect would occur even in aquifers with completely uniform porosity, permeability, and contaminant distributions, in the absence of dispersion, adsorption, diffusion-limited mass transfer rates, or other mechanisms often invoked to account for the right-hand tail on field curves for contaminant extraction. These mechanisms may be important, but it may not be possible to ascribe actual performance uniquely to one or more of these processes.

\section{STREAMLINE MODELS FOR VERTICAL VAPOR EXTRACTION WELLS}

In the previous section, we showed how contaminant extraction can be calculated from a streamline distribution as a function of cumulative total fluid extracted for a particular pattern and a very simple that is, uniform - initial distribution of contaminant within the pattern. We extended this approach to model advective contaminant transport in soil vapor extraction from single, isolated wells. This work was presented by one of the authors (R. W. Falta) at the AIChE 1992 Summer National Meeting in Minneapolis, MN (Falta, et al, 1992). A copy of the unpublished preprint is included as the first part of Appendix A.

The basic approach is as follows:

- Determine, analytically, the steady-state pressure distribution created in the porous medium by extracting gas from the well for the specific boundary conditions of interest.

- Using the Cauchy-Rieman equations, determine the normalized streamfunction as a function of position over the region of interest.

- Calculate the travel time distribution from the pressure solution by integrating along streamlines.

- Superimpose a streamfunction contour plot and a travel-time contour plot on a spatial plot of the contaminant distribution.

- Determine which contaminated elements reach the extraction well as a function of time.

Examples of contaminant concentration versus time in the extracted vapor are given in Figures 9, 12, and 14 of the preprint (see Appendix A). In the first two examples, the contaminant concentration is a monotonically decreasing function of time, since the initially contaminated region intersects the extraction well bore. In the latter exam $\Gamma$ ie, the concentration at first increases, then passes through a maximum, and finally decreases, due to a layered initial contaminant distribution causing a delay in breakthrough of contaminant from some regions.

The second part of Appendix A was written by R. W. Falta, and provides the algorithm and code listing for calculating the gas pressure distribution and the streamfunction distribution for a horizontal extraction well combined with a horizontal injection well, for potential application to the ISAS test at Savannah River. The next step in this work, which was not funded, would have been to extend the contaminant transport calculations illustrated for vertical wells in the first part of Appendix A to the horizontal well case, and include an initial contaminant distribution more or less like the SRID site. 


\section{EfFeCts of PeRmeability VARiation}

Although differing in important details, modeling groundwater or vadose zone remediation is conceptually similar to modeling enhanced oil recovery, in that both typically involve multi-phase, multicomponent fluid systems, transient flow and transport conditions, and complex chemical, physical, and even biological processes goveming the transfer of mass and energy among elements of the subsurface system (i.e., the aquifer or petroleum reservoir and its contained fluids) and between the system and its surroundings. Both types of processes involve the displacement of one fluid by another, and fluid displacement efficiency is profoundly affected by the spatial heterogeneity of the geologic media in which these displacements are conducted. Process efficiency, in turn, depends strongly upon fluid displacement efficiency.

Geologic media are generally much more heterogeneous than the porous materials used in chemical processing equipment, such as packed columns. One of the daunting problems in modeling fluid displacement processes in natural materials is the adequate characterization of their heterogeneity. The degree of success in predicting the flow and transport behavior of real systems depends upon how well the actual spatial variability of physical properties is approximated in the model, as well as upon how completely the physical and chemical processes important to process performance are represented. Since any model, no matter how complex, is an approximation, the central problem for modelers is to simplify the problem as much as possible without losing the predictive accuracy required by project objectives.

The degree of variability and its spatial scale depend upon the specific property being estimated. For example, the intrinsic permeability of geologic media can easily vary over five or six orders of magnitude even within distances of a few tens of feet or less, but the fractional porosity is physically constrained to lie between zero and unity. In turn, these different properties have varying impact on flow and transport through the system.

The petroleum industry has produced an enormous literature of laboratory investigations, field data, and model studies concerned with the injection of water, carbon dioxide, air, hydrocarbon gases, steam, polymer solutions, and surfactants into petroleum reservoirs, and the resulting production of oil, other native fluids, and injected substances. Much of this work has been focused on the prediction of oil recovery as a function of time, and frequent reality checks between models and experiments have been impartially imposed by economics. Hence, the analysis of petroleum production processes, and appropriate modification of the methods used, may accelerate the development of cost-effective characterization and modeling approaches for remediation projects.

In Appendix B, we summarize in detail the derivation of a simple "heterogeneity parameter" model described by Chesnut $e t$ al. (1979) for predicting (or extrapolating) the performance of waterfloods in depleted petroleum reservoirs. The approach is then extended to develop a model for contaminant removal from sediments by the extraction of contaminated air from a vadose zone or contaminated water from an aquifer. A similar analysis was used recently by Chesnut (1992) to define quantitatively, for heterogeneous systems, the concept of "groundwater travel time" introduced by the Nuclear Regulatory Commission as one criterion for determining the suitability of a site as a potential repository for high-level nuclear waste.

Appendix $\mathrm{C}$ contains details of fitting either field data or the results of more complex model calculations with the heterogeneity parameter model. In the following sub-sections, we first summarize briefly the general model and the physical interpretation of the parameters for the different displacement processes (waterflooding, vapor extraction, and pump and treat), then give the results of fitting the Savannah River ISAS and P\&T data. 


\section{Heterogeneity Parameter Models}

These models all start with the concept of an ideal linear "piston-like" displacement described verbally in Section II and mathematically in Appendix B. This concept is represented pictorially in Figure IV.1 as a square pulse with both width and height equal to unity.

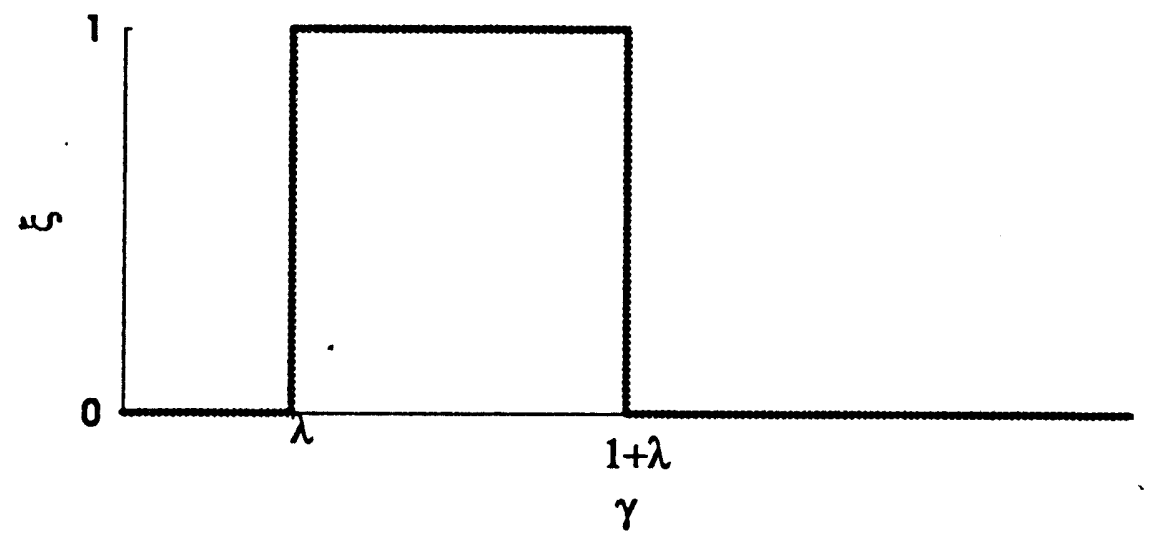

\section{Figure IV.1. Ideal normalized removal rate, $\xi$, versus normalized fluid throughput, $\gamma$. The normalized delay parameter is $\lambda$.}

In the case of waterflooding, $\xi$ is the ratio of the oil production rate to the water injection rate, and $\gamma$ is the ratio of cumulative water injected to the amount of movable oil present at the start of water injection. The delay parameter accounts for the water that must be injected to displace free gas from the reservoir before any oil can be displaced. If there is no free gas, $\lambda$ is equal to zero, oil production starts immediately, and all the oil is displaced when $\gamma=1$. Both injected and produced fluid rates and cumulatives are used in the analysis, since both are typically measured.

In the case of contaminant removal, $\xi$ is the ratio of contaminant concentration measured at the extraction well, divided by the average initial concentration of contaminant in the carrier fluid within the initially contaminated fraction of the capture volume. The delay parameter $\lambda$ is the ratio of the volume of initially clean sediment to the total capture volume, assuming that the contaminant must be transported through the clean sediment before reaching the extraction point. The variable $\gamma$ is the ratio of cumulative carrier fluid extracted to the amount that would have to be extracted to remove all of the contaminant at its average initial concentration. Only extracted contaminant concentration and cumulative carrier fluid extracted are used in the analysis, since no fluids are injected in some applications.

Figure IV.2 shows the normalized cumulative removal, $\Xi$, as a function of normalized throughput, $\gamma$. For waterflooding, $\Xi$ is the cumulative fraction of the waterflood movable oil recovered, and for contaminant extraction, it is the cumulative fraction of the initial contaminant mass recovered. In both cases, a value of unity corresponds to complete recovery. 
This ideal process is completely specified by three parameters (which have different physical meanings for the different processes considered):

1. A normalizing factor for the rate of production;

2. A normalizing factor for the cumulative production (essentially the total mass of oil or contaminant initially in the system);

3. A delay parameter.

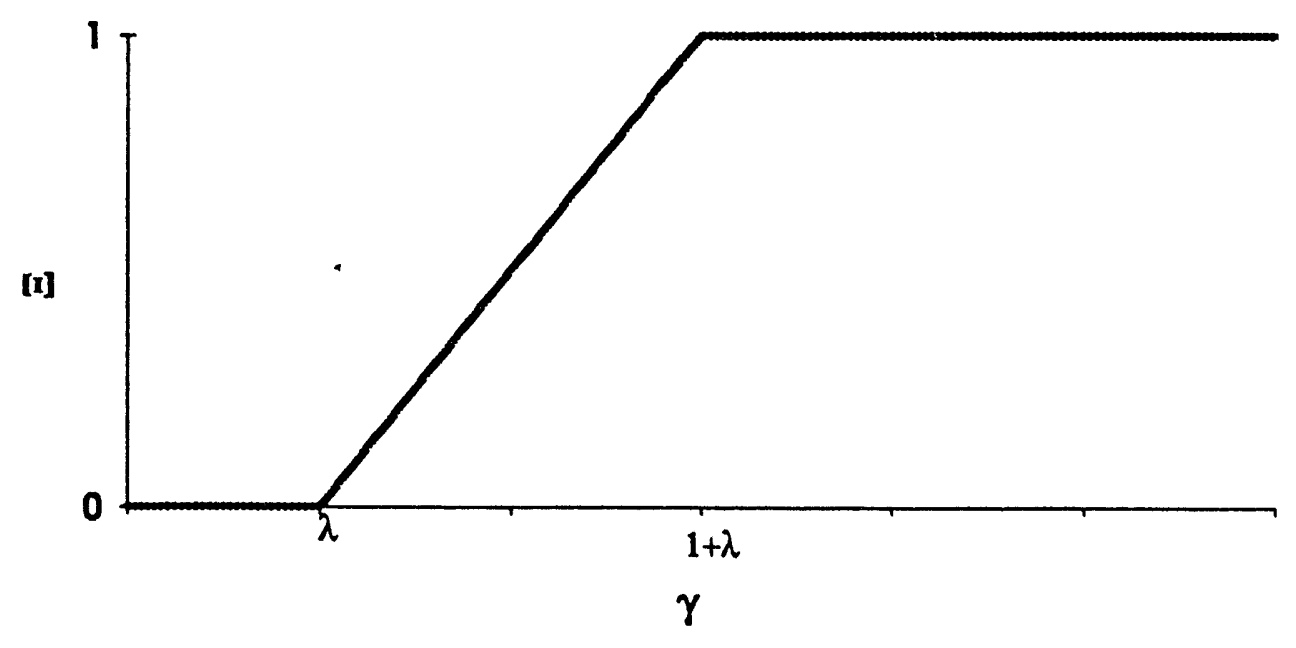

Figure IV.2. Ideal normalized cumulative removal, $\Xi$, versus normalized fluid throughput, $\gamma$. The delay parameter is $\lambda$. Note that the cumulative removal reaches unity at a throughput of $1+\lambda$, and is zero for a throughput of $\lambda$ or less.

For heterogeneous systems (under certain simplifying assumptions given in Appendix B), it is only necessary to introduce one additional parameter, $\sigma$, which characterizes the degree of heterogeneity. For $\sigma=0$, the system exhibits the ideal behavior sketched in Figs. IV.1 and IV.2. We showed previously the normalized response curves for several values of $\sigma$, with $\lambda=0$, in Fig. II.1.

The equations for normalized removal rate and normalized cumulative mass removal as functions of throughput are as follows:

$$
\xi(\gamma)=\Phi\left\{\frac{\ln \left[\frac{\gamma}{\lambda}\right]+\frac{\sigma^{2}}{2}}{\sigma}\right\}-\Phi\left\{\frac{\ln \left[\frac{\gamma}{\lambda+1}\right]+\frac{\sigma^{2}}{2}}{\sigma}\right\}
$$




$$
\Xi(\gamma)=\gamma \cdot \xi(\gamma)+\{1+\lambda\} \cdot \Phi\left\{\frac{\ln \left[\frac{\gamma}{1+\lambda}\right]-\frac{\sigma^{2}}{2}}{\sigma}\right\}-\lambda \cdot \Phi\left\{\frac{\ln \left[\frac{\gamma}{\lambda}\right]-\frac{\sigma^{2}}{2}}{\sigma}\right\}
$$

where $\Phi(x)=\frac{1}{\sqrt{2 \pi}} \int_{-\infty}^{x} e^{-x^{2} / 2} d x$ is the normal probability integral.

In applying these equations to contaminant extraction, note that $\xi=c / c_{0}$, where $c_{0}$ is the average initial contaminant concentration in the carrier fluid (water for a pump and treat system or air for a vacuum extraction system). The normalized cumulative removal, $\Xi$, is equal to $M / M_{o}$, where $M_{0}$ is the total initial mass of contaminant.

The normalized throughput, $\gamma$ is $Q /\left(M_{0} / c_{0}\right)$, where $Q$ is the cumulative volume of carrier fluid extracted. Note that if the concentration of contaminant in the carrier fluid were to remain constant until all the contaminant had been extracted, the total carrier fluid volume extracted at complete removal would equal $M_{0} / c_{0}$.

For carrier fluid extraction at a constant average rate $q$, time can be introduced by noting that $Q=q t$, and a dimensionless time can be defined as $\tau=t\left(q c_{0} / M_{0}\right)=t / t_{0}$. The characteristic time, $t_{0}=M_{o} /\left(q c_{0}\right)$, is the time which would be required to remove all the contaminant at concentration $c_{o}$ with a constant carrier fluid extraction rate, $q$.

The total capture volume is $(1+\lambda) V_{c}$, where $V_{c}$ is the contaminated bulk volume within the capture zone, and $\lambda V_{c}$ is the uncontaminated part of the bulk volume within the capture zone. The delay parameter $\lambda$ is included to allow for extraction projects in which the contaminant is not immediately adjacent to the outflow boundary when extraction begins.

Up to this point, the heterogeneity parameter model makes no distinction between air as the carrier fluid (as in vapor extraction) or water as the carrier fluid (as in pump and treat). The difference arises in the relationship of the initial contaminant mass inventory to the contaminated bulk volume and the initial average concentration in the carrier phase.

For vapor extraction, we show in Appendix B that the initial contaminant inventory is given by

$$
\begin{aligned}
M_{c o} & =V_{c}\left[C_{v o} \phi S_{v}+C_{b} \phi\left(1-S_{v}\right)\right] \\
& =V_{c} C_{v o} \phi S_{v}\left[1+\frac{C_{b}\left(1-S_{v}\right)}{C_{v o} S_{v}}\right]
\end{aligned}
$$

In Eq. (IV.3), the substitution $C_{v o} \equiv c_{0}$ has been made to emphasize that the initial contaminant concentration refers to the mobile phase. In this equation, $V_{c}$ is the bulk volume of contaminated sediment, $\phi$ is the average porosity, and $S_{v}$ is the average gas (or vapor) saturation as a fraction of the pore volume. $C_{l o}$ is the concentration in the (immobile) aqueous phase, assumed to be in equilibrium with the average initial vapor phase concentration. 
In the second line of Eq. (TV.3), the factor in front will be recognized as the total initial contaminant mass in the mobile carrier phase, and the term in brackets is just unity plus the ratio of contaminant mass in the stationary aqueous phase to contaminant mass in the mobile phase. This will be recognized as the retardation coefficient arising from the partitioning of contaminant between air and water in the vadose zone.

This result is easily generalized by inspection to include the partitioning of contaminant among several stationary phases (e.g., linear adsorpion on the solid phase), so long as the total mass can be represented as a retardation coefficient times the mass in the mobile phase. In particular, the application of the equations to pump and treat is obvious, replacing $\phi S_{v}$ by $\phi$ and the term in brackets by $1+R_{s}$ to allow for partitioning between the mobile aqueous phase and the stationary solid phase.

Finally, note that the retardation coefficient does not appear explicitly in the dimensionless equations: only the total mass inventory and the average mobile phase initial contaminant concentration are needed to normalize the performance data. However, it is required if one wishes to attempt an a priori forecast of contaminant extraction from independent estimates of capture volume, porosity, contaminant concentration, etc. Note that the ratio of water saturation to gas saturation is particularly important in determining $\mathrm{R}$ for vadose-zone remediation. For clays, the water saturation is typically near unity, and the retardation coefficient may be quite large even with no solid phase adsorption. This model does not explicitly account for the occurrence of non-aqueous phase liquids (NAPLs), although they could be accommodated in modest amounts in the sense that they would show up in the initial contaminant inventory and average concentration.

Figures IV. 3 through IV.9 show the sensitivity of the normalized contaminant extraction rate curves to the parameters $\lambda$ and $\sigma$. These parameters determine the shapes of the plots, on $\log -\log$ axes, of field contaminant concentration vs. cumulative throughput. The initial concentration and contaminant inventory parameters merely shift the curves along the vertical and horizontal axes, respectively. Hence, these plots could be used as type curves for matching similar plots for field data, although the procedure given in Appendix C, using a spreadsheet, is easier because the comparison of plots can be done on a computer screen instead of with graph paper.

It can be shown, by some tedious manipulation of Eq. (IV.1), that the function $\xi(\gamma)$ has a maximum at a normalized throughput equal to $\gamma^{*}$, where

$$
\gamma^{*}=e^{-\sigma^{2} / 2} \sqrt{\lambda(1+\lambda)},
$$

and the maximum value of $\xi$ is given by

$$
\xi_{\max }=2 \Phi\left[\frac{\ln \left(\frac{1+\lambda}{\lambda}\right)}{2 \sigma}\right]-1
$$

Note that the point at which the maximum occurs shifts to the right with increasing $\lambda$, and to the left with increasing $\sigma$. The magnitude of the maximum decreases monotonically with increasing $\sigma$ and with increasing $\lambda$. The normalized concentration versus throughput curves become progressively broader as $\sigma$ increases. 


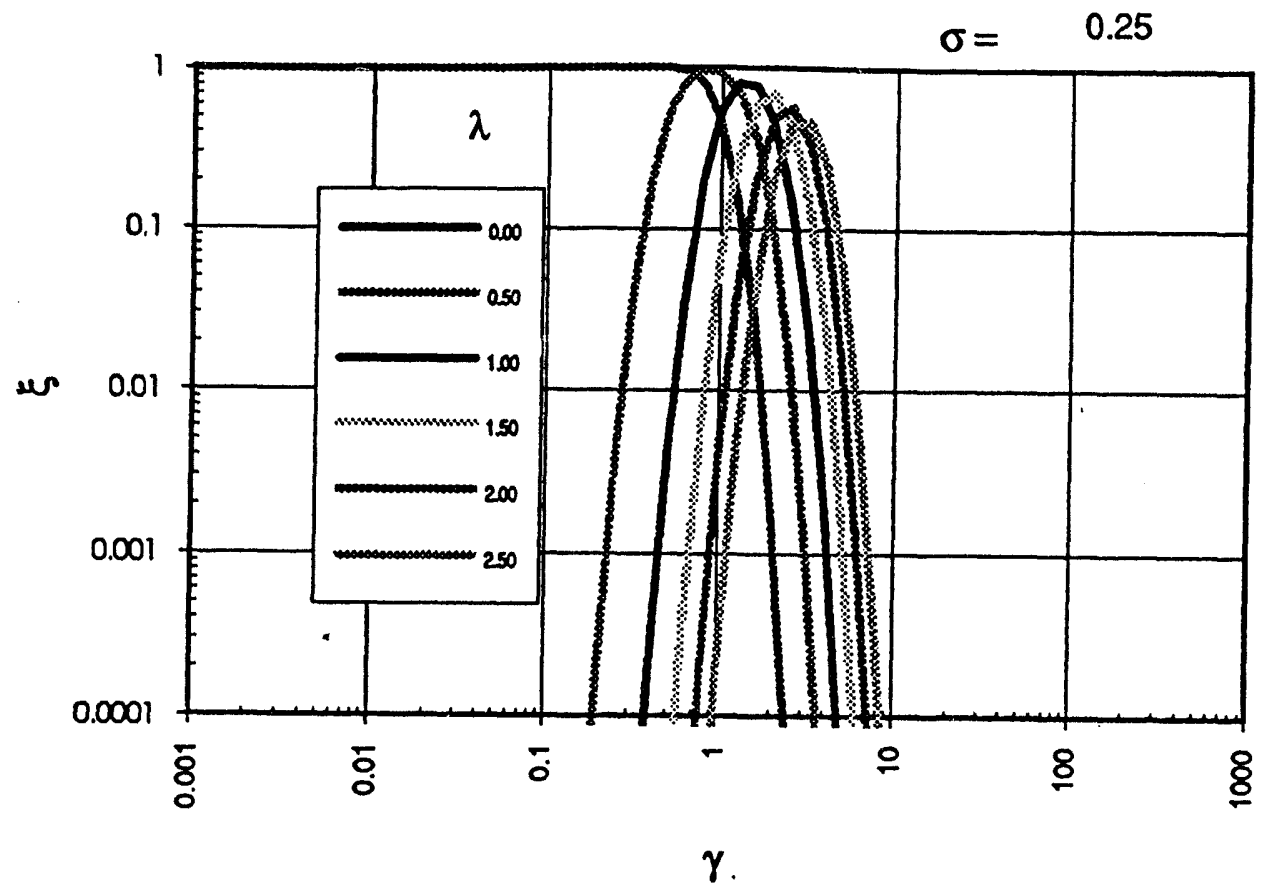

Figure IV.3. Variation with $\lambda$ of normalized contaminant concentration vs. $\gamma$ for
$\sigma=0.25$

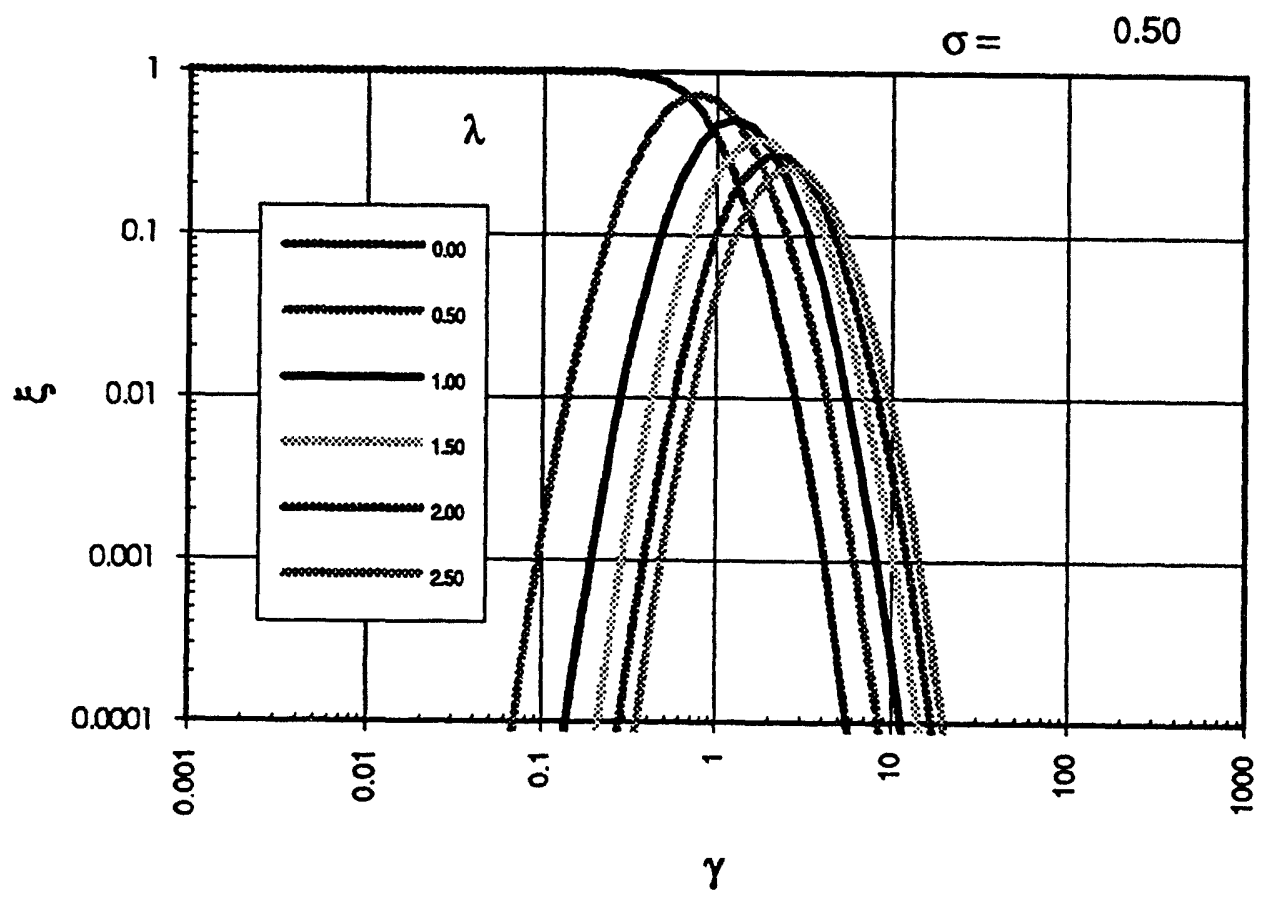

Figure IV.4. Variation with $\lambda$ of normalized contaminant concentration vs. $\gamma$ for $\sigma=0.50$. 


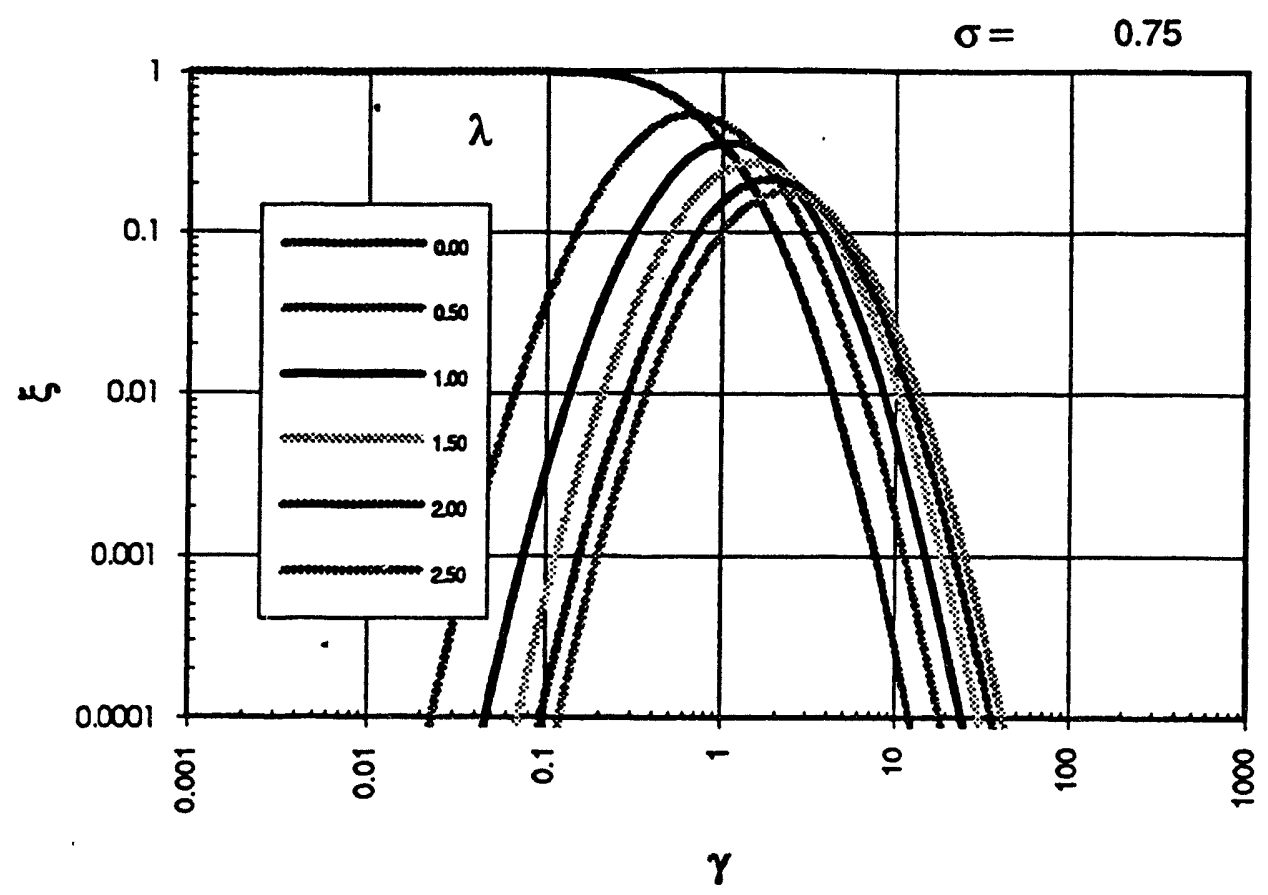

Figure IV.5. Variation with $\lambda$ of normalized contaminant concentration vs. $\gamma$ for $\sigma=0.75$.

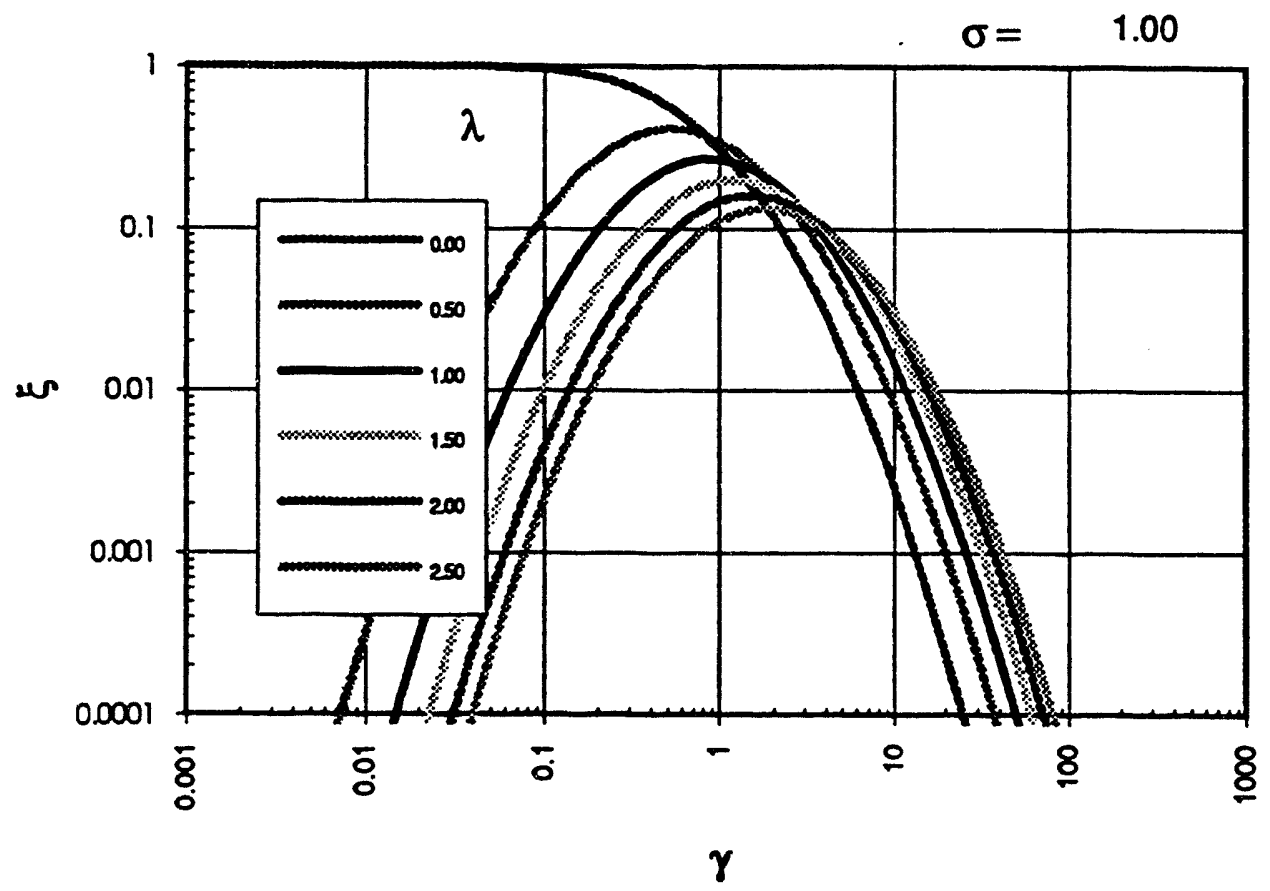

Figure IV.6. Variation with $\lambda$ of normalized contaminant concentration vs. $\gamma$ for $\sigma=\mathbf{1 . 0 0}$. 


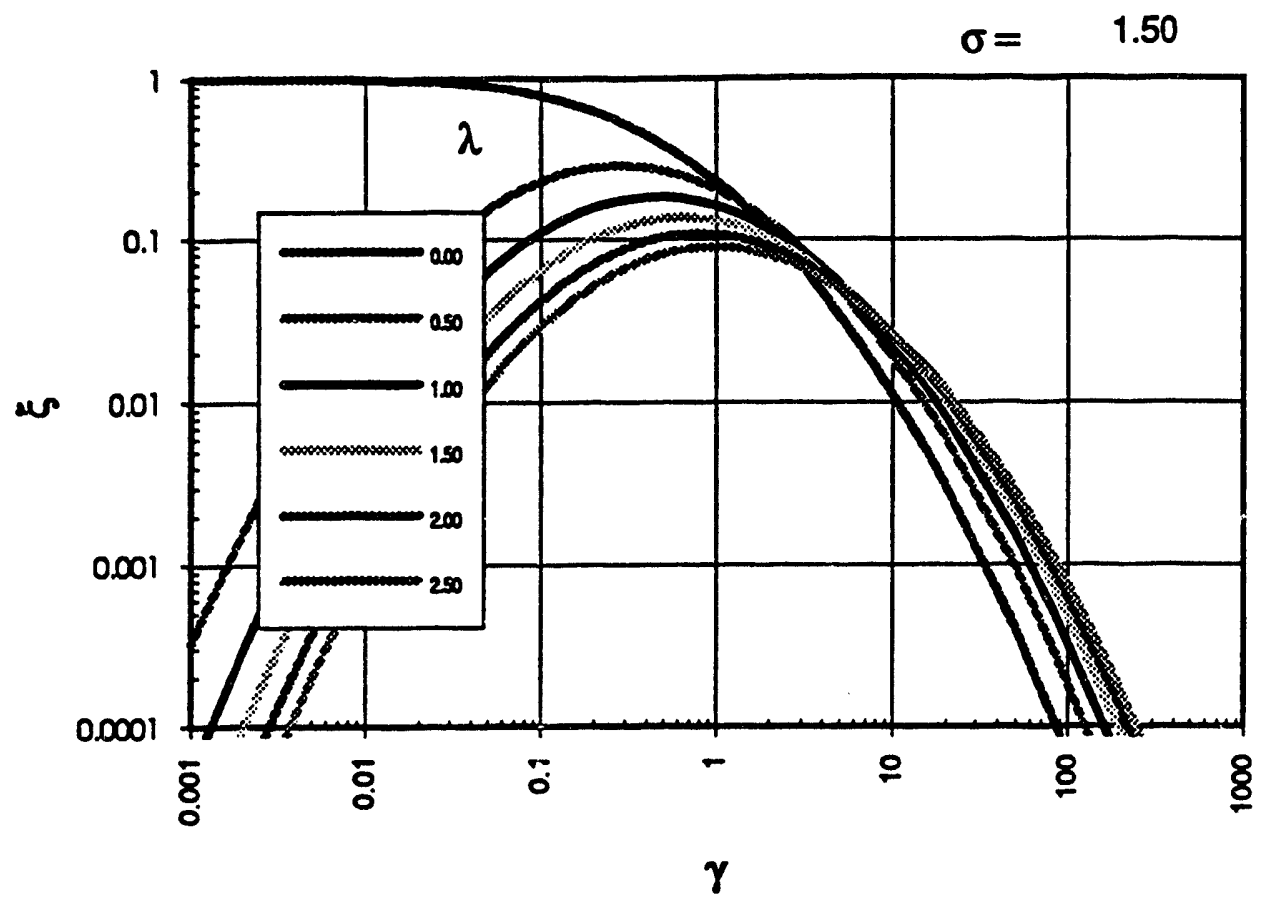

Figure IV.7. Variation with $\lambda$ of normalized contaminant concentration vs. $\gamma$ for $\sigma=1.50$.

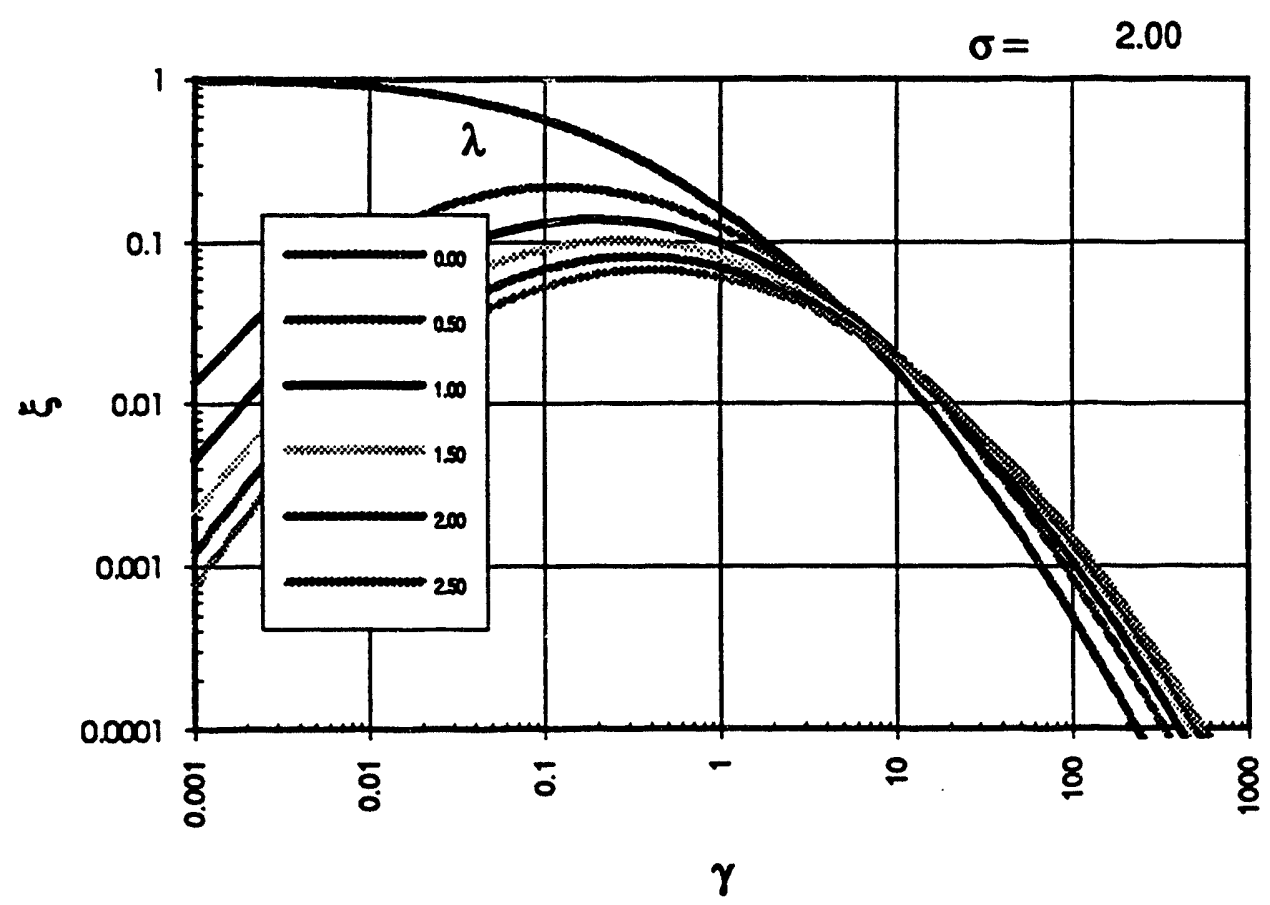

Figure IV.8. Variation with $\lambda$ of normalized contaminant concentration vs. $\gamma$ for $\sigma=2.00$. 


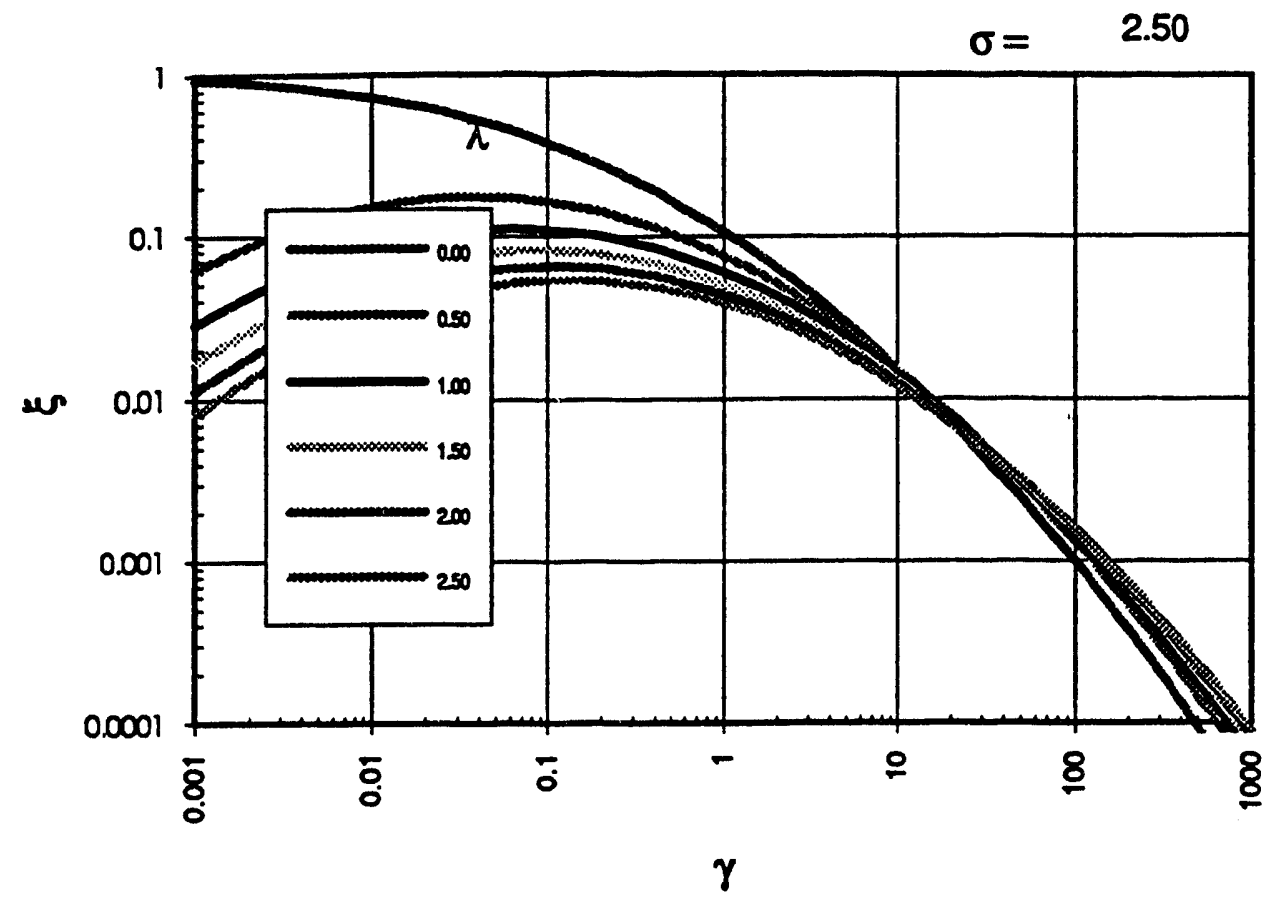

Figure IV.9. Variation with $\lambda$ of normalized contaminant concentration vs. $\gamma$ for $\sigma=2.50$.

\section{APPLICATION to SR In SITU AIR STRIPPING TeST}

Two horizontal wells, AMH-1 and AMH-2, were drilled and completed in September and October, 1988, for use in the Air-Stripping Phase of the Savannah River Integrated Demonstration Project for the Removal of VOCs at Non-Arid Sites. The extraction well, AMH-2, has a screened interval of approximately 200 feet entirely within the vadose zone, and the injection well, AMH-1, has a screened interval below the water table of about 310 feet.

Vacuum extraction at approximately 580 SCFM from AMH-2 started July 27, 1990 and continued, with minor interruptions, until December 13, 1990 (Looney, et al., 1991). During part of the test period, air was injected at various rates below the water table through AMH-1, to test the possibility of air-stripping VOCs from groundwater in situ while using vacuum extraction to remove contaminants from the vadose zone.

During the first 21 calendar days of operation, a total of approximately $2696 \mathrm{lb}$. of VOCs was removed. For the first 15 days, only the extraction well was used. Injection into AMH-1 at 65 SCFM began on day 16 , and the rate was increased to 140 SCFM on day 28 and to 270 SCFM on day 69 . Injection stopped on day 113 , and extraction continued to day 140 . Note that all time references are in calendar days elapsed from the start of the test. Approximately 120 days of actual operating time were achieved, accomplishing the removal of about 15900 pounds of VOCs.

As a preliminary step in applying the heterogeneity parameter model to vapor extraction in heterogeneous systems, concentrations of TCE and PCE in the gas extracted from AMH-2 were plotted versus hours of net operating time (i.e., with down time subtracted), using a log-log scale, in Fig. IV.10. The resulting curves bear only a slight resemblance to the normalized model curves previously shown in Fig. II.1 for $\lambda=0$. However, the sequence of curves given by Figs. IV.3 through IV.9 shows that the shape of the 
concentration versus time plots can be varied considerably by appropriate choice of the parameters, and that relatively high values of $\sigma$ will be needed to obtain curves spanning several orders of magnitude in time.

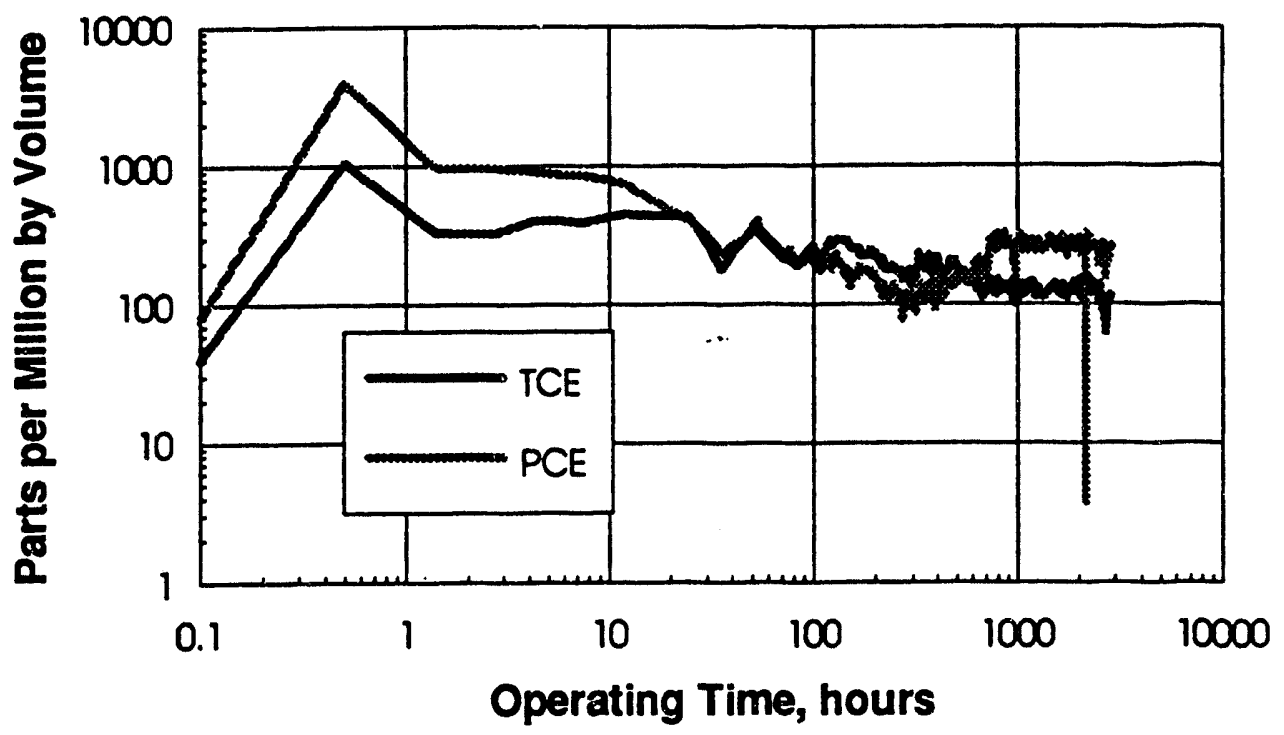

Figure IV.10. Contaminant concentrations (ppm by volume) measured in gases extracted from horizontal well AMH-2 during the Savannah River Integrated Demonstration of In-Situ Air Stripping.

A complication arises from changing operating conditions during the course of the test. Many simplifying assumptions were made in the derivation of the model equations. One of the more important of these is the implicit assumption that the pore volume affected by the extraction well remains constant over time. This is probably justified if operating conditions are held constant and the flow properties of the system are such that a pseudo-steady state is reached in a time short compared to the duration of the operation.

There were several significant changes during the operation of the In-Situ Air Stripping Test. Injection at 65 SCFM was started 342.5 operating hours after extraction began, was increased to 170 SCFM at 655.0 hours, and then increased again to 270 SCFM at 1511.6 hours. Injection was terminated at 2261.6 hours, and extraction continued until 2879.1 hours. These changes altered the capture volume of AMH-2.

To attempt fitting the data, we decided to concentrate first on the initial 342.5 hours, when the operation was a pure vapor extraction process. Spreadsheet PCE_TCE.XIS was developed to import the field concentration vs. time data, accept input values for the model parameters, calculate the normalized concentration vs. normalized cumulative extraction from Eq. (IV.1) for these values, and graph both the field data and model calculations on the same log-log plot so they can be compared visually.

Examination of the model equations shows that there are four parameters to be determined: $\lambda, \sigma, C_{0}$, and $t_{0}$. The first two are dimensionless parameters which affect the shape of the concentration response curve when plotted on log-log paper. The second pair are scale factors (a characteristic concentration and a characteristic time, respectively) which translate the log-log response curve parallel to the vertical and horizontal axes without changing its shape. 
Since the field vapor-phase contaminant concentration data are given in ppm by volume, and any consistent units can be used for the normalized concentration appearing in the left hand side of Eq (TV.1), it is convenient to choose the vertical scale factor $c_{0}$ to match the field data as reported, then convert the final results to mass per unit volume as follows:

$$
C_{\text {no }}=10^{-6} c_{0}\left(\frac{M W}{359.1}\right)
$$

In Eq. (IV.6), $c_{0}$ is in ppm by volume, $M W$ is the molecular weight in $\mathrm{lb}_{m}$ per $\mathrm{lb}$.-mole, and the constant in the denominator is the number of standard cubic feet per lb.-mole of an ideal gas. The concentration $C_{\text {vo }}$ will then be in $\mathrm{lb}_{m}$ per SCF.

The parameter $t_{0}$ for translation along the horizontal axis maps the normalized cumulative extraction into a corresponding value for the independent throughput variable. Since the extraction rate was treated as a constant in deriving the model equations, either time or cumulative extraction can be used as an independent variable for plotting the response curve. Time is somewhat more convenient if the injection rate does not vary much. If it does vary significantly, it will often be advantageous to use the cumulative extraction as the horizontal scale. We chose to use time because the extraction rate was almost constant except when the system was down, and down time was subtracted from elapsed time. Hence we seek a scale factor by matching the plots such that

$$
t=t_{0} \gamma
$$

Since $\gamma$ is dimensionless, it is obvious that $t_{0}$ is a characteristic time. From the discussion of heterogeneity parameter models following Eqs. (IV.1) through (IV.3), recall that

$$
t_{0}=\frac{M_{0}}{q c_{0}}
$$

After determining values for the two scale factors $c_{0}$ and $t_{0}$ by matching the field data, and calculating $C_{v_{0}}$ from Eq. (IV.6), the total initial mass inventory can calculated from

$$
M_{0}=C_{w_{0}} t_{0} q
$$

In using Eq. (IV.9), care must be taken to use consistent units. For time in hours and concentration in $\mathrm{Ib}_{m}$ per $\mathrm{ft}^{3}$., the volumetric extraction rate must be in cubic feet per hour. The mass inventory will then be obtained in pounds mass.

Figure (TV.11) shows a plot from the spreadsheet for fitting the model to the early time data for TCE concentration in the extracted gas. The agreement between the model and field data is visually quite good before air injection started. There is an increasing spread between the model and the field results as the operation continues, indicating that air injection may be sweeping contaminant to the extraction well which would not be extracted otherwise. No attempt was made to match the cumulative contaminant mass withdrawal directly.

Figure (IV.12) shows the match for the early-time PCE data. Again, the match is quite good until about the time that air injection began, with an even more pronounced departure afterward than is shown by the TCE data. 


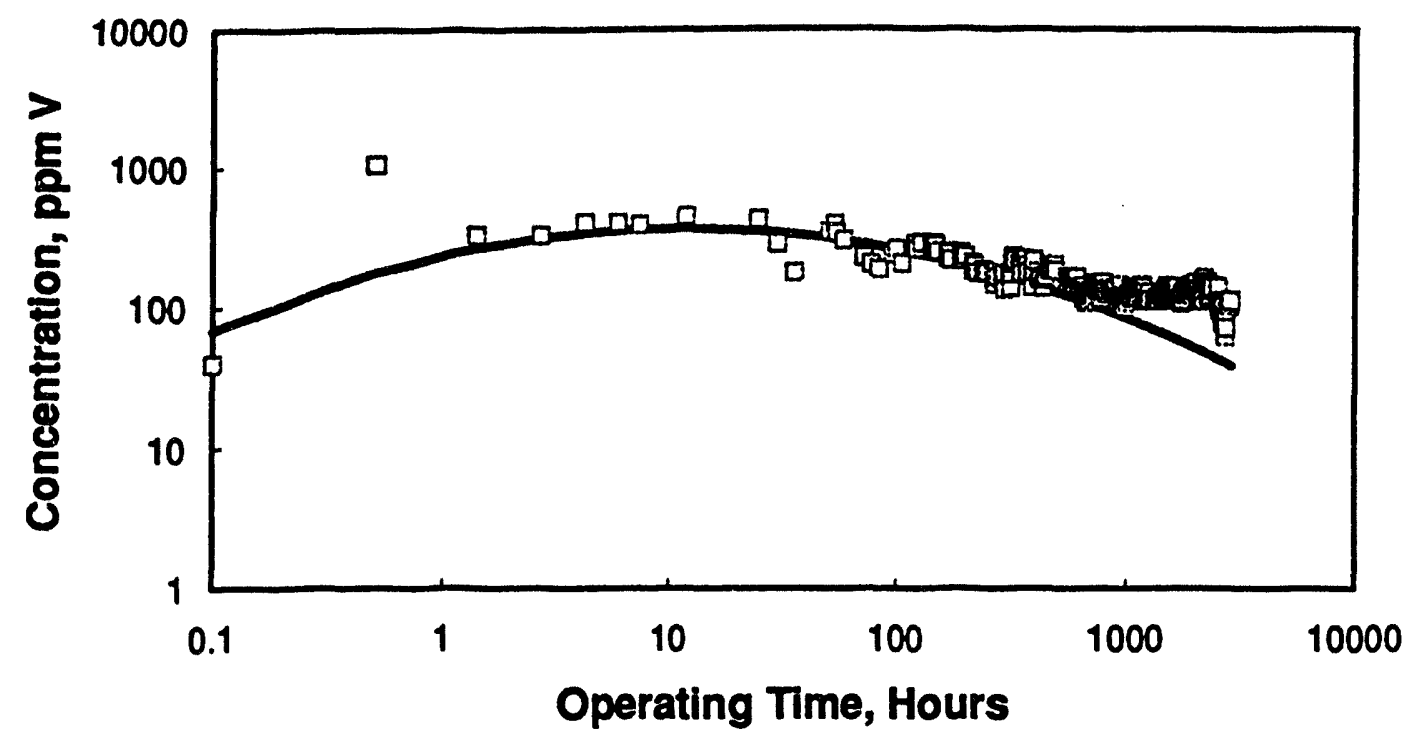

Figure IV.11. Fit of early-time TCE extraction data to the heterogeneity parameter model. The squares are the data points, and the smooth curve is calculated from Eq. (IV.1) with $\lambda=0.10, \sigma=2.5, C_{0}=1000$, and $t_{0}=\mathbf{8 0 0} \mathrm{hr}$.

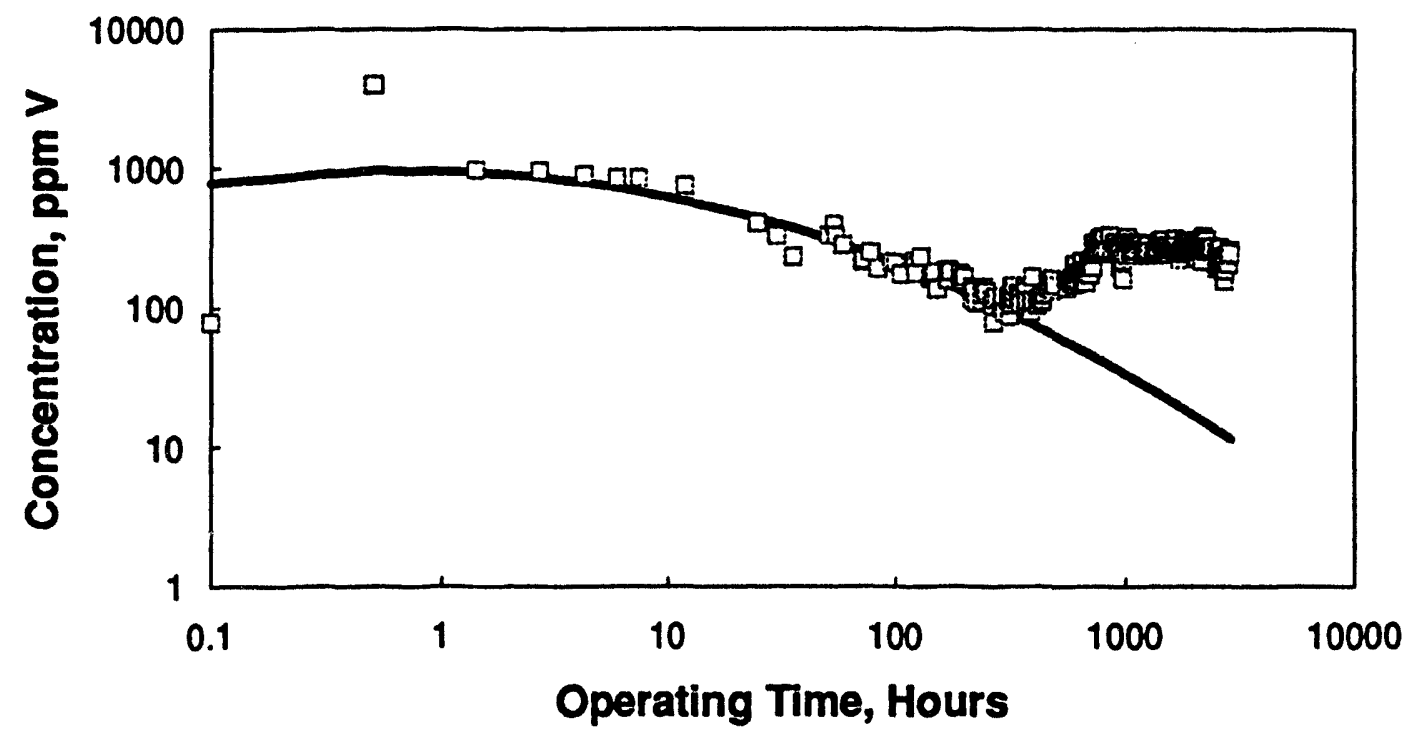

Figure IV.12. Fit of early-time PCE extraction data to the heterogeneity parameter model. The squares are the data points, and the smooth curve is calculated from Eq. (IV.1) with $\lambda=0.01, \sigma=2.5, C_{0}=1500$, and $C_{1}=150$. 
The parameters from the early-time match were used in a second spreadsheet to calculate concentrations of TCE and PCE in ppm for each time they were measured, and the calculated values were subtracted from observed values to obtain residuals. These residuals were assumed to result from the sweep of additional contaminant inventory to the extraction well as a result of injection, and the effect of injection rate was ignored. The spreadsheet was then used to fit these residual concentrations with a second set of parameter values for each contaminant. The results for TCE are shown in Fig. (IV.13); those for PCE are shown in Fig. (TV.14).

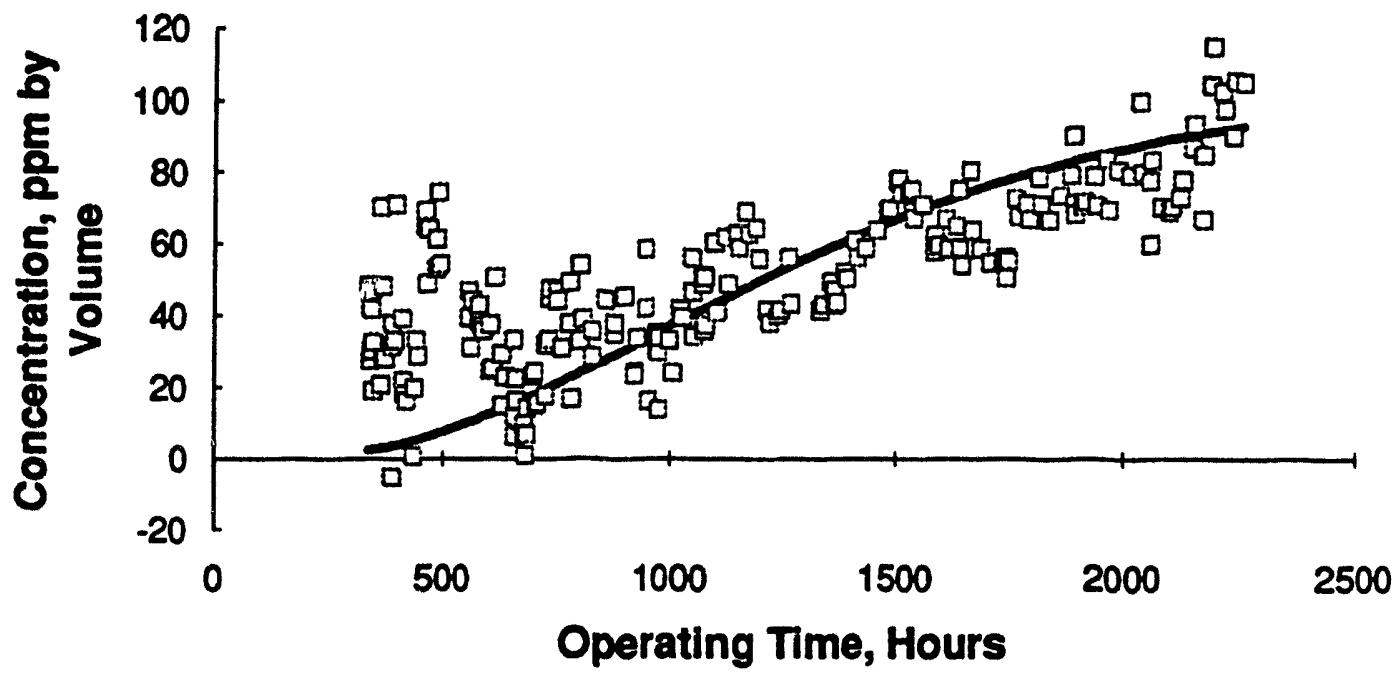

Figure IV.13. Residual TCE concentrations compared to model calculations with parameters chosen to fit the residual data. The parameter values are shown in Table IV.1.

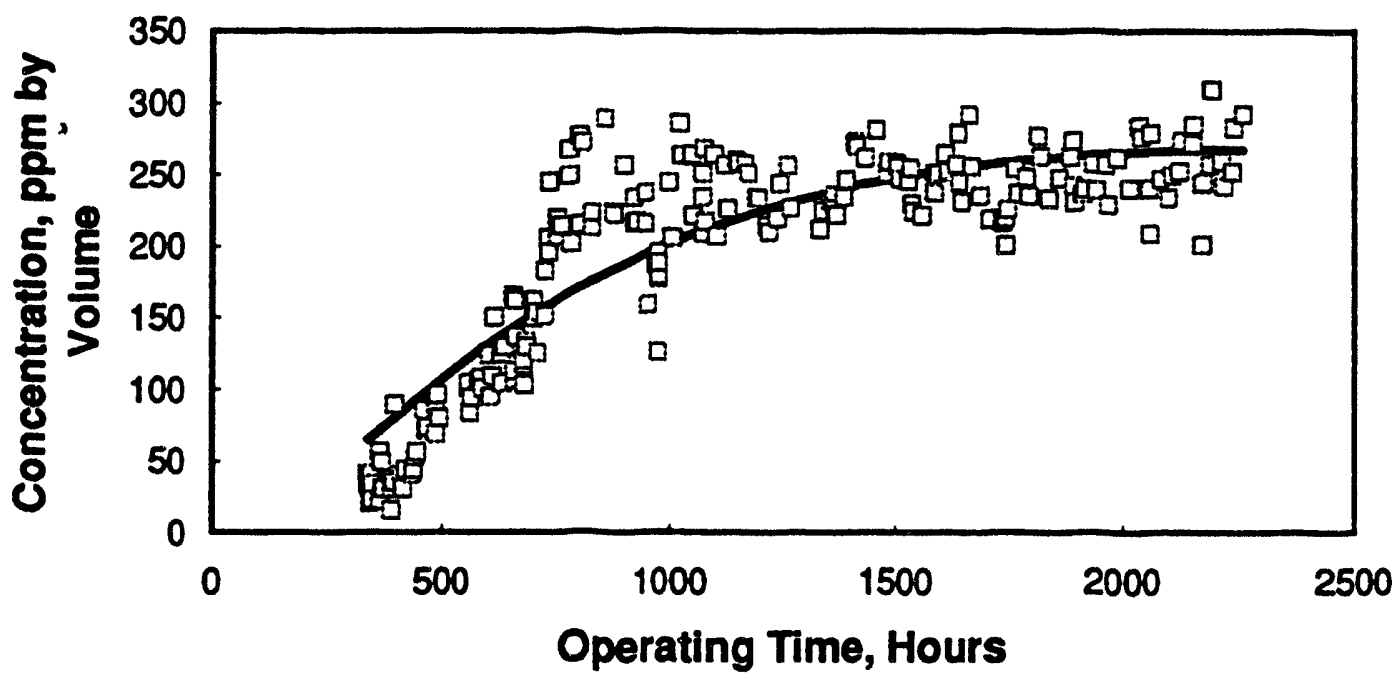

Figure IV.14. Residual PCE concentrations compared to model calculations with parameters chosen to fit the residual data. The parameter values are shown in Table IV.1. 
Although there is considerable scatter in the data, the overall agreement shown in Figs. IV.13 and IV.14 is reasonably good. More emphasis was given to obtaining a match at later time than at early time, since the injection conditions were held constant for a longer interval. Parameter values for the early-time (VE) and late-time (AS) models are given in Table IV.1 for both TCE and PCE.

Table IV.1. Summary of heterogeneity model parameters obtained by fitting field data for the concentration of VOCs in the gas extracted during the Savannah River Integrated Demonstration of In-Situ Air Stripping. The total TCE inventory is estimated to be about 21,000 lb., and the PCE inventory estimate is just below $60,000 \mathrm{lb}$.

\begin{tabular}{|c|c|c|c|c|}
\hline Parameter & TCE $_{(\mathrm{VE})}$ & $\mathrm{TCE}_{(\mathrm{AS})}$ & $\mathrm{PCE}_{(\mathrm{VE})}$ & $\mathrm{PCE}_{(\mathrm{AS})}$ \\
\hline$\lambda$ & 0.10 & 8.00 & 0.01 & 0.5 \\
\hline$\sigma$ & 2.5 & 0.8 & 2.5 & 1.1 \\
\hline$c_{0, p p m V}$ & 1000 & 1700 & 1500 & 700 \\
\hline$t_{0, h r}$ & 800 & 500 & 150 & 5000 \\
\hline$M_{0, \text { bb. }}$ & 10186 & 10823 & 3616 & 56246 \\
\hline
\end{tabular}

The spreadsheet was then used to calculate concentrations vs. time for each set of parameters for each contaminant, and the resulting pairs of concentration values for TCE and PCE were added together at each time to produce total concentration vs. time curves for each contaminant. The results are shown and compared with field data in Fig IV.15. The agreement is quite good.

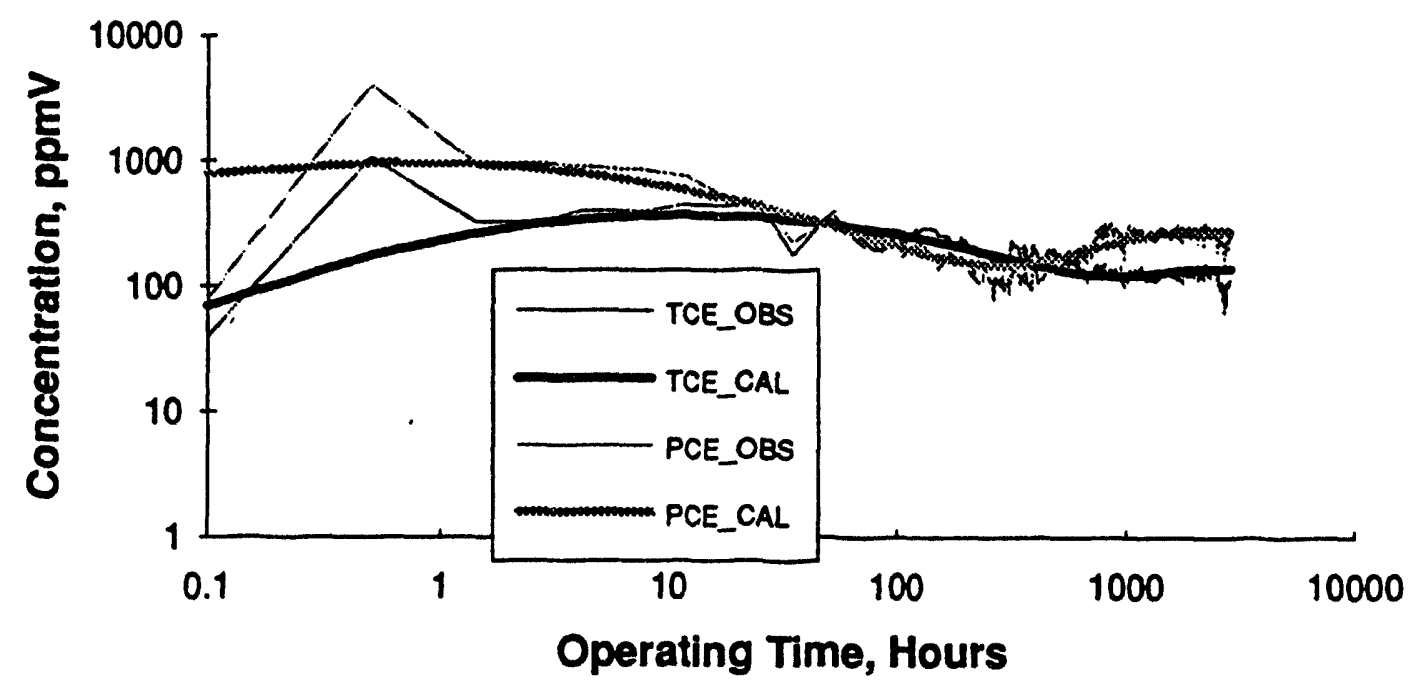

Figure IV.15. Final results of fitting field data as the sum of two model curves for each contaminant. The parameter values are given in Table IV.1. 
Finally, Eq. (IV.2) was used to calculate the cumulative mass extracted for each component for both parameter sets, and the results for each separate component were added together. The resulting cumulative extraction plots are given in Figs. IV.16 and IV.17.

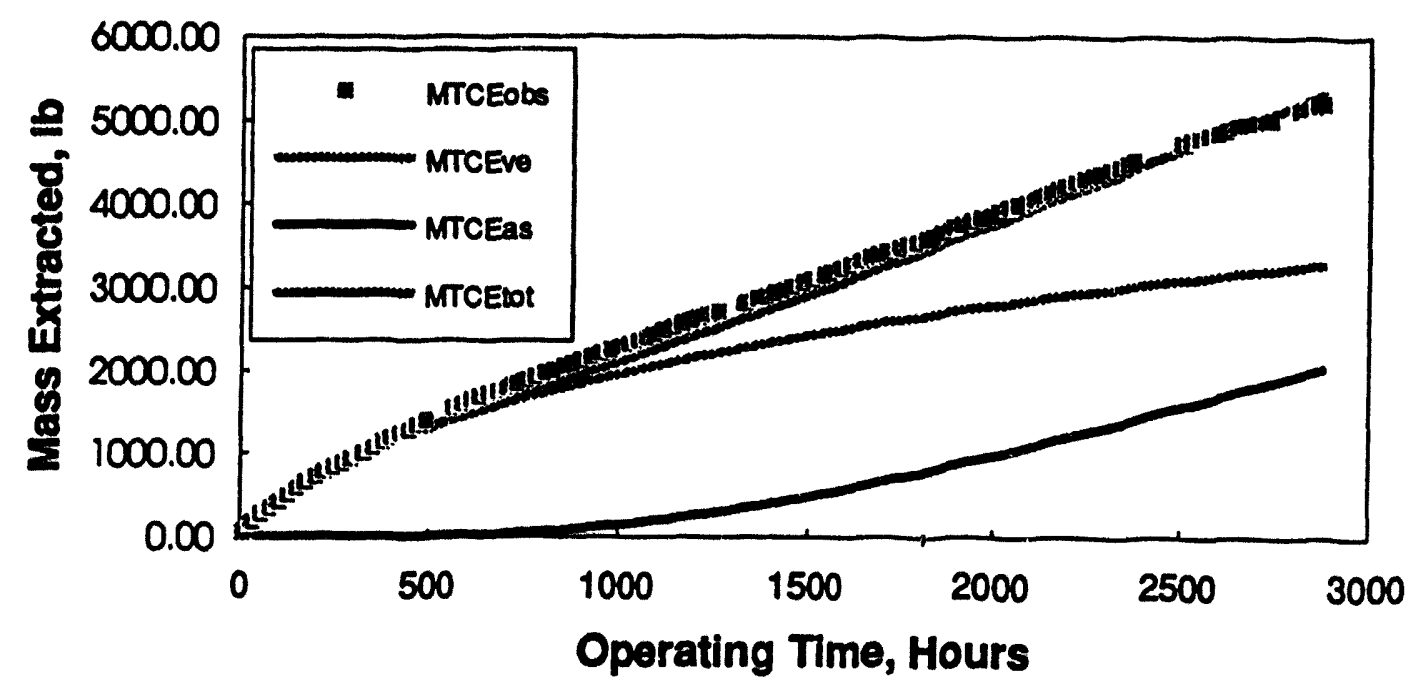

Figure IV.16. Comparison of model and field results for cumulative TCE extraction. The lower curve is an estimate for extraction assisted by air stripping, and the next curve above is for extraction alone. The upper smooth curve is the total extraction predicted by the model, which is in close agreement with the data points (shown as squares).

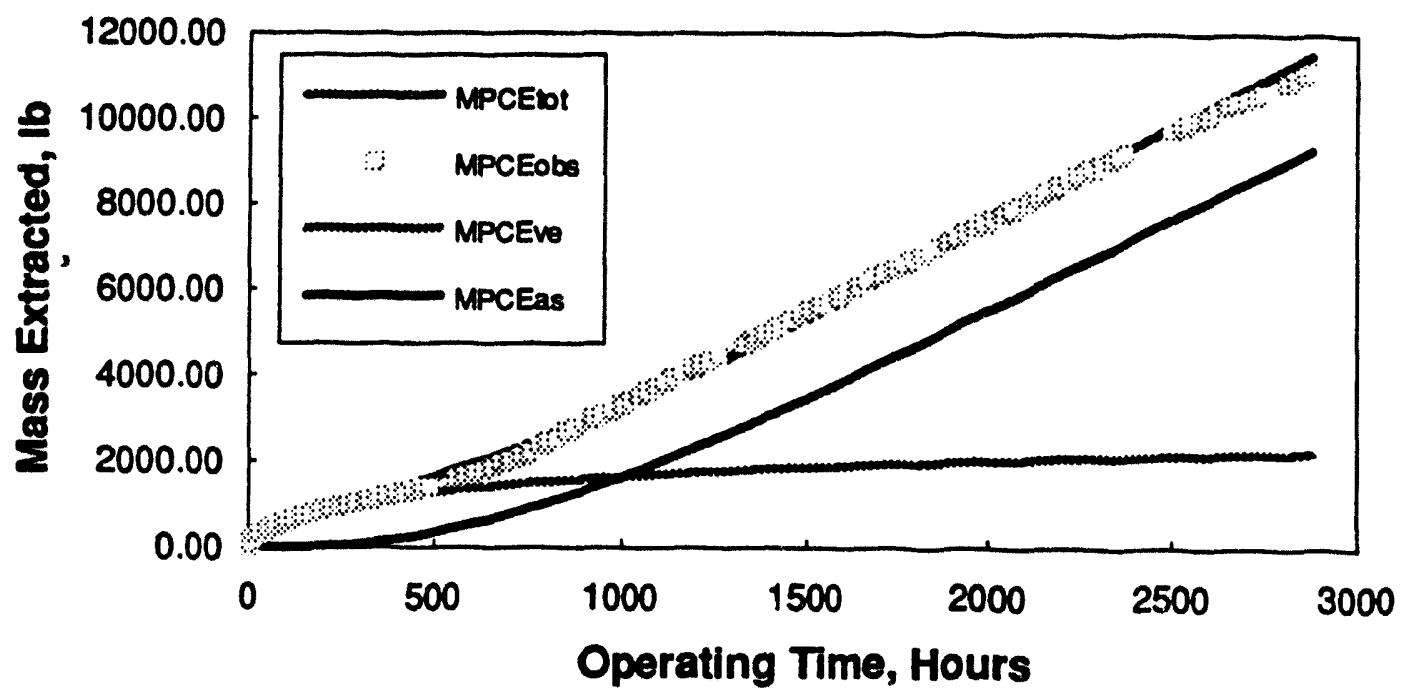

Figure IV.17. Comparison of model and field results for cumulative PCE extraction. The curves are similar to those for TCE in Fig. IV.16, except that the curve including the effect of air-stripping crosses the extraction-only curve at about 1000 hours and accounts for most of the PCE by the end of the test. 
Cumulative extraction estimates calculated from the measured concentration data and the nominal extraction rate are $5218 \mathrm{lb}$. for TCE and $11149 \mathrm{lb}$. for PCE. Comparable model results are $5323 \mathrm{lb}$. and $11511 \mathrm{lb}$., respectively. The model estimate is about $2 \%$ above actual for TCE and about $3 \%$ above actual for PCE, which is remarkably close agreement. However, it should be quickly noted that there are a number of parameters to adjust, and this agreement may merely reflect the existence of enough degrees of freedom to match almost any data set.

If we tentatively accept the total mass inventory indicated by the model parameters chosen to fit the data, the VOC recovery of about $16000 \mathrm{lb}$. is only about $20 \%$ of the indicated initial contaminant inventory of approximately $81,000 \mathrm{lb}$.

We emphasize that this document is a progress report in the development of an approach for quantifying the effects of field-scale heterogeneities on remediation processes. It is not a finished product, and much work remains to be done even with Savannah River data before taking the results too seriously. However, the general approach has worked well when applied to waterflooding reservoirs with enough core data to determine accurate a priori estimates for the model parameters (pore volumes, saturations, and the variance of intrinsic permeability), provided that the flood pattern was left unchanged over most of the project life. In such cases, there is little difference between the parameter values determined from core data and the values obtained by least-squares fitting of the production response curves. Each waterflood response curve requires only a single set of parameters.

In the curve-fitting exercise for the ISAS experiment at Savannah River, it was necessary, conceptually, to associate two different pore volumes with each contaminant concentration vs. time curve in order to match the model to the field data. An analogous situation sometimes occurs in highly stratified petroleum reservoirs, when the system responds as two distinct layers connected only through the wellbores. It is then easy 10 justify the addition of two response curves to obtain the total production.

In the present case, this justification is not so easy. While the introduction of a mass source into the system by injecting air will certainly change the flow patterns, the treatment of the subsequent behavior as a continuation of the early time performance with a second response added is basically an ad hoc assumption and needs to be investigated further. Simulators will be very useful in this investigation, because it is then possible to specify exactly the distribution of the important physical variables, in contrast to a real system.

However, at present, it is interesting to speculate on the meaning of the results obtained above. One of the difficult problems in analyzing the Savannah River experience is the separation of the effects of the horizontal well geometry from the effects of air injection on the overall VOC recovery performance. Until we can develop a clear rationale for this separation, it is difficult to transfer the technology.

Another problem arises in comparing in-situ air stripping with other remediation technologies which have been applied at the site. The pump and treat system has been in operation for more than seven years, but it encompasses a much larger area. For this reason, it would be expected to perform more poorly than a small-scale system located near a "hot spot" of contamination. The vertical-well vacuum extraction test conducted before the horizontal system was developed was also in the hot spot area, but the test was much too short to allow a good comparison to be made with in-siw air stripping.

If the model results can be taken seriously, the performance with and without air injection can be calculated directly from the two sets of model parameters given above. The model result for total VOC extracted during the entire test is $16834 \mathrm{lb}$., of which $5501 \mathrm{lb}$. is attributed to vacuum extraction alone and $11333 \mathrm{lb}$. is attributed to the increase resulting from air injection. This represents a $200 \%$ increase in the amount of contaminant removed during the test, compared to the amount that would have been removed had air not been injected. Table IV.2. compares the calculated results for TCE and PCE with the corresponding field data. 
Table IV.2. Comparison of the calculated mass of TCE and PCE removed during the Savannah River Integrated Demonstration Test with field data. All amounts are in pounds.

\begin{tabular}{|c|c|c|c|c|c|c|c|}
\hline \multicolumn{4}{|c|}{ TCE } & \multicolumn{4}{c|}{ PCE } \\
\hline Field & $\begin{array}{c}\text { Model } \\
\text { VE }\end{array}$ & $\begin{array}{c}\text { Model } \\
\text { AS }\end{array}$ & $\begin{array}{c}\text { Model } \\
\text { Total }\end{array}$ & Field & $\begin{array}{c}\text { Model } \\
\text { VE }\end{array}$ & $\begin{array}{c}\text { Model } \\
\text { AS }\end{array}$ & $\begin{array}{c}\text { Model } \\
\text { Total }\end{array}$ \\
\hline 5218.12 & 3288.09 & 2034.68 & 5322.77 & 11149.23 & 2212.58 & 9298.48 & 11511.06 \\
\hline
\end{tabular}

\section{APplication to SR PUMP aNd Treat Data}

As noted earlier in this section, the heterogeneity parameter equations in dimensionless form do not explicilly depend upon whether the extraction process under consideration uses air or water as the carrier fluid. The difference lies entirely in the relationship of the initial contaminant mass inventory, $M_{0}$, and the initial average contaminant concentration in the carrier fluid, $c_{0}$, to other parameters of the subsurface system. The general relationship is

$$
M_{0}=V_{p c} S_{m} c_{0}(1+K)
$$

Here, $V_{p c}$ is the contaminated pore volume occupied by the carrier fluid, $S_{m}$ is the saturation of carrier fluid, and $K$ is the ratio of contaminant mass in all the stationary phases to contaminant mass in the mobile carrier phase. The term in parentheses will be recognized as the retardation coefficient, defined in more detail in Appendix B. Note that, for pump and treat, the only partitioning involved is between water and the sediment grains, while for vadose zone remediation by vapor extraction, the partitioning between vapor and immobile water must be considered as well.

The normalized cumulative throughput variable, $\gamma$, can be calculated from either of the following two equalities:

$$
\begin{aligned}
\gamma & =Q / Q_{0}, \text { or } \\
\gamma & =t / t_{0}
\end{aligned}
$$

Recall that $Q_{0}$ is the cumulative carrier fluid that would have to be extracted to remove all the contaminant if its concentration at the outflow boundary of the system were to remain constant, and $t_{0}$ is the time that would be required for contaminant removal at a constant carrier fluid extraction rate. The former quantity is thus scen to be a characteristic amount of carrier fluid, and the latter is obviously a characteristic time.

Since the field data normally reported are measured contaminant concentrations versus time, it is ustally most convenient to use time as the independent variable for fitting the heterogeneity parameter model. For the Savannah River pump and treat operation, we used data for the first thirteen months, which were readily available to us in Colven et al. (1987). These data comprise the average monthly concentrations of TCE and PCE in the total air stripping tower influent, the number of operating hours each month, and the average flow rate in gallons per minute. These data were entered into a spreadsheet, PTFIT2XILS, developed to determine a set of heterogeneity model parameters giving reasonable agreement between calculated and observed concentrations as functions of time. Details of the curve-fitting process are given in Appendix C. The resulting parameter values are given in Table IV.3. 
Table IV.3. Heterogeneity model parameters for the Savannah River Pump and Treat Project determined by fitting average monthly concentration versus operating time observed during the first 13 months of operation.

\begin{tabular}{|c|c|c|}
\hline Parameter & Value for TCE & Value for PCE \\
\hline$\lambda$ & $1.00^{* 10^{-5}}$ & $1.00^{*} 10^{-5}$ \\
\hline$\sigma$ & 2.5 & 2.5 \\
\hline$c_{0,}$ ppb & 36400 & 15750 \\
\hline so $_{\text {, hours }}$ & 167000 & 2750000 \\
\hline$M_{0}$, pounds & $1,156,000$ & 824,000 \\
\hline
\end{tabular}

Figure IV.18 compares the calculated and observed concentrations as functions of operating time. In judging the quality of fit, one should be aware that some of the reported PCE concentration data were apparently not accurate. Of the thirteen monthly values reported, nine were tabulated as "less than" the figure given. No discussion was included in Colven et al. (1987) on errors in the reported concentrations.

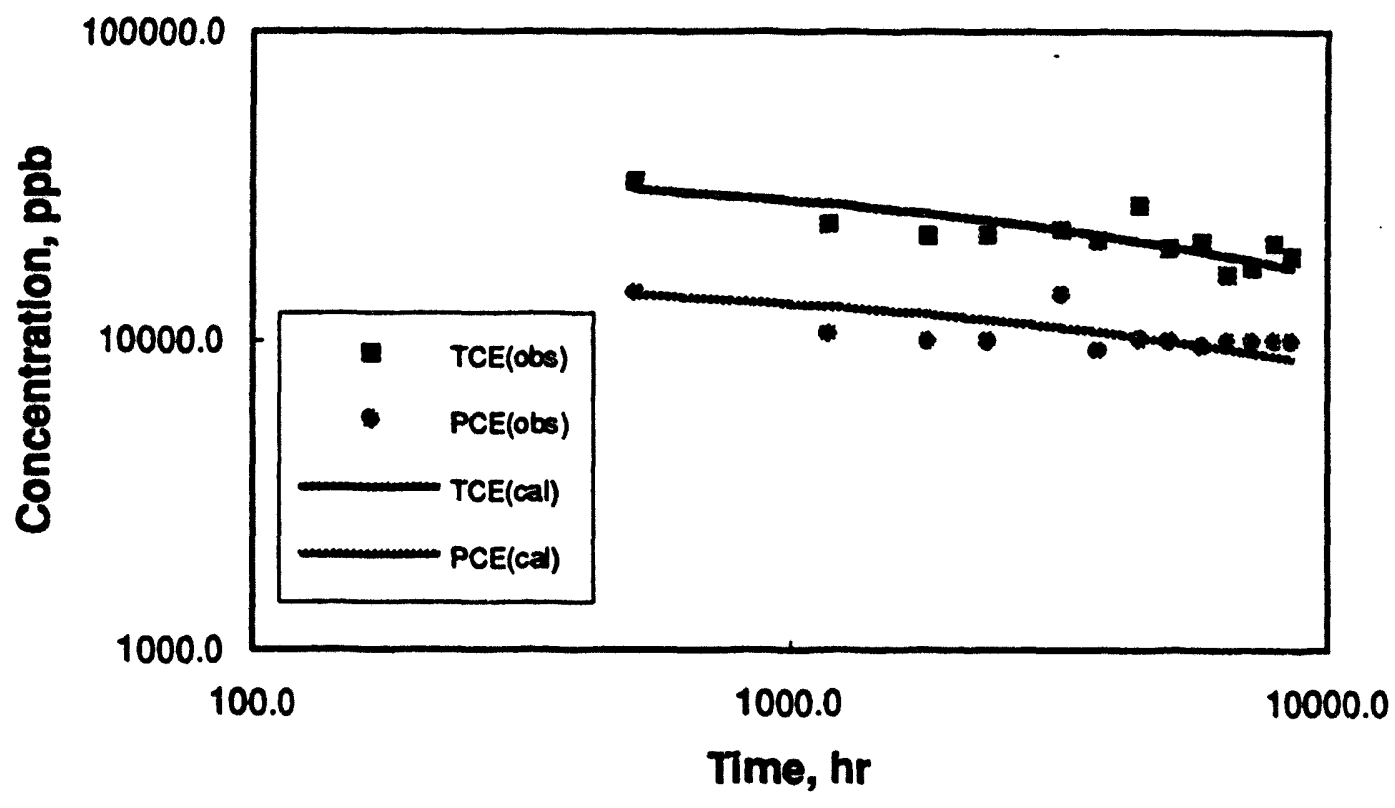

Figure IV.18. Heterogeneity parameter model fit for Savannah River Pump and Treat data, September 1985 through September 1986 (from Colven et al, 1987)

In fitting the data, we used the value of $\sigma$ that provided the best fit to the early-time ISAS data (i.e., 2.5), reasoning that in these two pure extraction processes the flow pathways might be geometrically similar predominantly parallel to the stratification - even though the ISAS test used horizontal wells and extracted from intervals lower in the stratigraphic column. Also, $\lambda$ was held constant at a small value because the maximum observed concentrations occurred near the beginning of the project. Additional work with the model is needed to determine whether equally good or better agreement could be obtained with other values of $\sigma$ and $\lambda$. 
According to the model, the initial inventory of TCE within the volume affected by the pump and treat operation was $1,156,000 \mathrm{lb}$., and the initial inventory of PCE was $824,000 \mathrm{lb}$., or a total of $1,156,000+824,000=1,980,000 \mathrm{lb}$. This is much higher than the range of 260,000 to $450,000 \mathrm{lb}$., based on characterization data, given in Colven et al. (1987). However, according to an oral presentation by Dr. Dawn Kaback during a site tour briefing in March 1992, a total of $114,000 \mathrm{~kg}$. (251,300 lb.) had been removed by June 1991, and the average monthly concentration was over $11,000 \mathrm{ppb}$., compared to an initial concentration of approximately $47,100 \mathrm{ppb}$. Since the actual cumulative contaminant mass extracted is almost equal to the lower limit of the range given by Colven et al., and the concentration is still very high, it seems quite likely that the actual contaminant inventory originally present is significantly higher than the initial estimates. Whether it is actually anywhere near the estimate obtained from the heterogeneity parameter model fit remains to be determined. However, the value is not excluded by comparison with the $13,000,000$ pounds of solvents used at the site, as reported in the site tour briefing.

After obtaining these parameter values, we then developed a second spreadsheet, PT4CAST.XIS, for calculating contaminant recovery vs. time, assuming that the water pumping rate remains constant, for the future as well as for the initial thirteen month period used in fitting the data to the model. The spreadsheet results are tabulated in Appendix $C$.

One check on the quality of the data fit is a comparison of cumulative contaminant recovery during the 1.06 years of operating time used to fit the concentration vs. time data. The calculated recovery of TCE is $36,807 \mathrm{lb}$., and the calculated recovery of PCE is $17713 \mathrm{lb}$., giving a total of $54,520 \mathrm{lb}$., or about 1.9\% more than the total of $53,486 \mathrm{lb}$. reported by Colven et al. (1987). Of course, close agreement should be expected, since, although matching the estimated total contaminant mass removed was not explicitly used to fit the data, any reasonable representation of the concentration vs. time plots should work over short time intervals. Figure IV.19 shows the model "forecast" for a 10-year period from September 1985.

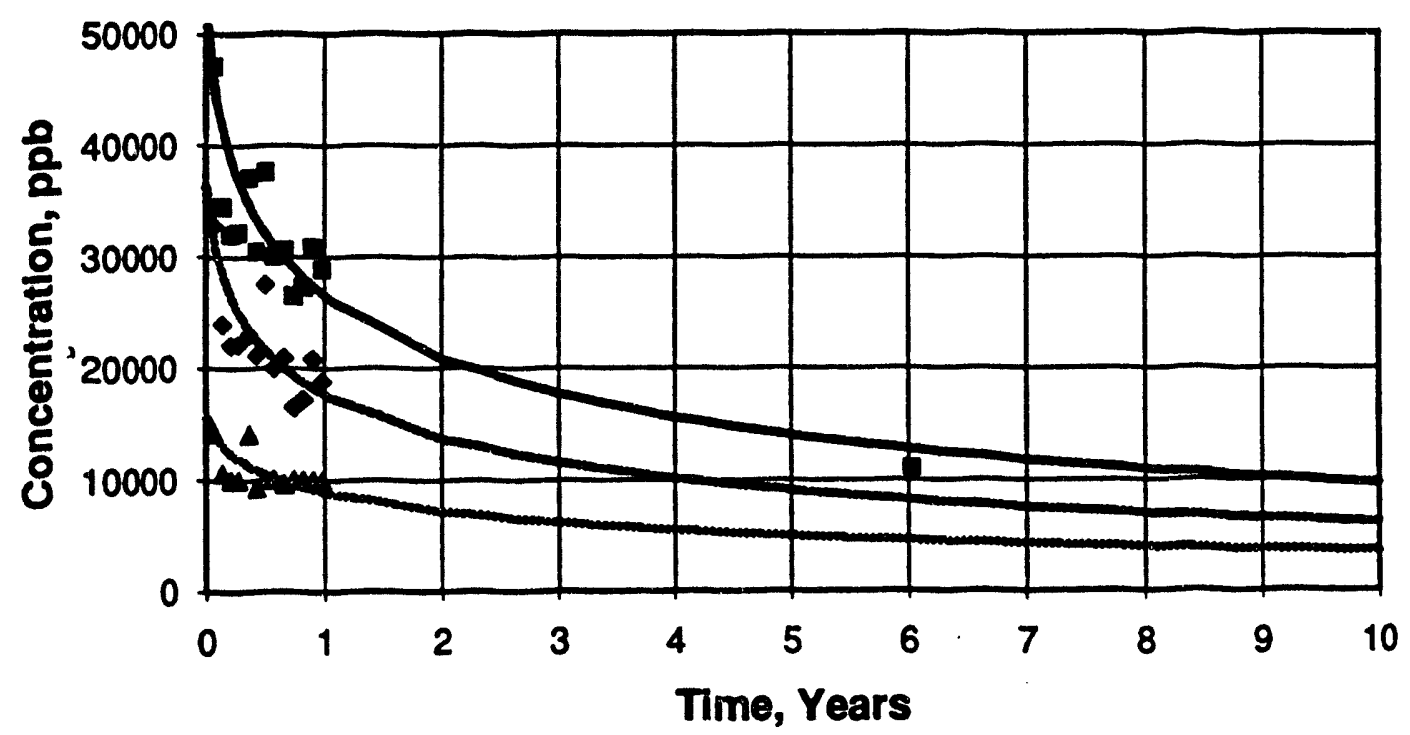

Figure IV.19. Comparison of model calculations and field data for the Savannah River P\&T project. The upper curve is for total VOCs, the middle curve is for TCE, and the lower one is for PCE. 
Comparison of these calculated values with actual experience during the seven years of operation would provide a very good test not only of the model but of the sensitivity of its parameters to the amount of actual performance data used in the fitting process. Perhaps an even more stringent test would be provided by fitting the data for each of the eleven individual extraction wells used in the P\&T project. However, these data were not available to us as of the date of this report, and this more detailed comparison is deferred indefinitely.

At present, we can only compare the forecast with corresponding values presented at the site tour briefing. The calculated VOC concentration after 6 years of operating time at a pumping rate of $380 \mathrm{gpm}$. is about $12700 \mathrm{ppb}$., or about $15 \%$ above the concentration reported at the briefing. The calculated VOC mass removed is $185,700 \mathrm{lb}$., or about $26 \%$ below the amount reported at the briefing. These errors do not seem to be excessive considering the limited amount of data used in determining the model parameters. Chesnut et al. (1978) mentioned that 18 months of data were required to get a reasonably accurate forecast for the performance of a waterflood over 20 years, and pointed out that waterflood model parameter values were essentially unchanged in adding data beyond 36 months. It would be of considerable interest to explore the sensitivity of model parameters to the amount of pump and treat data used in the fitting process.

Figure IV.20 shows the forecast extended to 500 years from September 1985. This plot dramatically illustrates the potential effect of heterogeneity on the time required for clean up. Note that calculated TCE and PCE concentrations are both well above $100 \mathrm{ppb}$. at 500 years, based on the current pumping rate. If this model is even roughly correct, there may not be any practical (i.e., affordable) method for reducing contaminant concentrations to the $10 \mathrm{ppb}$. range. Note that the characteristic times for TCE and PCE are $167,000 \mathrm{hr}$. and $275,000 \mathrm{hr}$., respectively, or about 20 years and 34 years. Hence, only a few decades would be required to achieve clean-up in a homogeneous system, in contrast to hundreds of years if the system is as heterogeneous as this curve fitting exercise suggests.

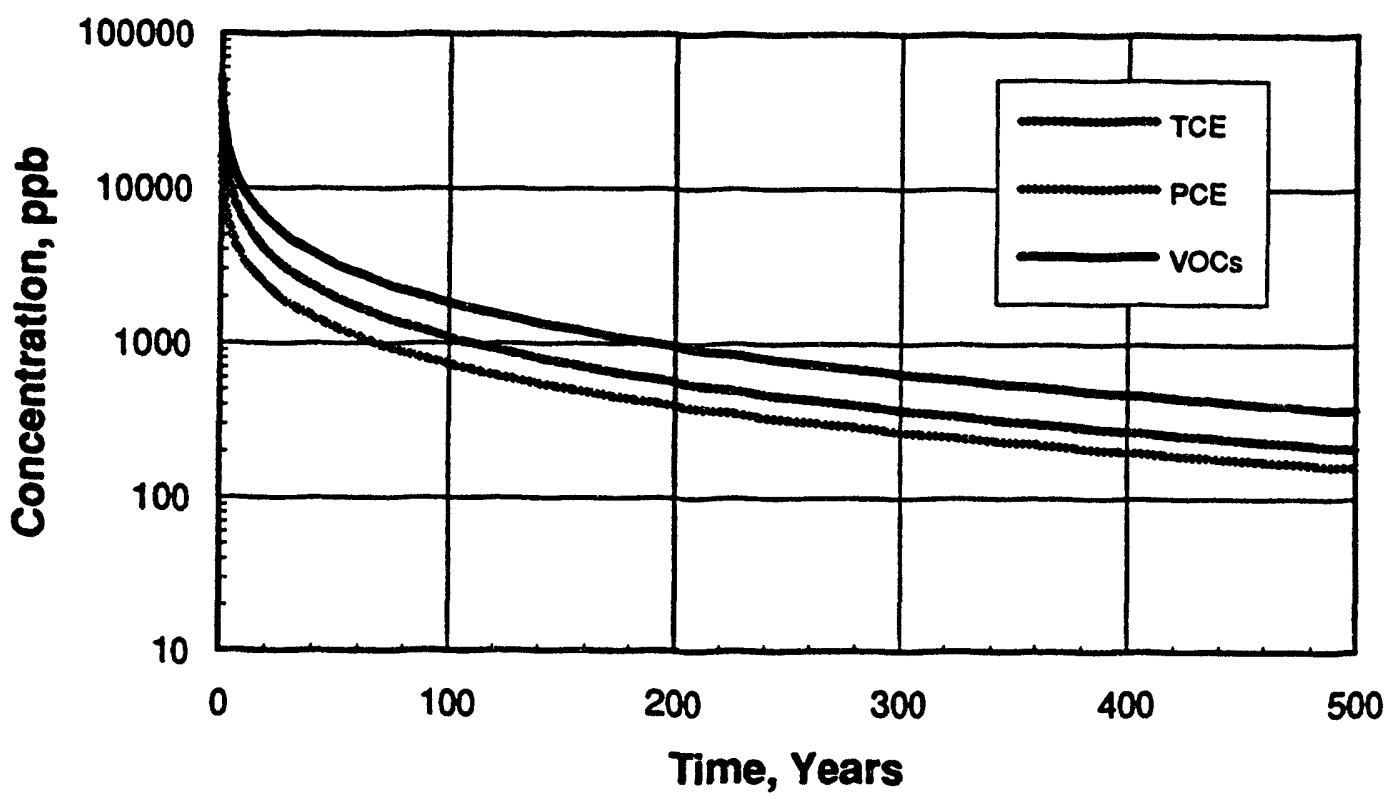

Figure IV.20. Heterogeneity model extrapolation for the Savannah River Pump and Treat project. 


\section{Field DATa}

\section{Well Completions}

Well completion information summarized in this section was given in Kaback et. al. (1989), for casing and tubing data, and in Looney et. al. (1991), for piezoneter (PZ) wbe positions. Table V.1 contains the data for the ISAS injection well, and Table V 2 gives similar information for the extraction well

Table V.1 Completion details of injection well AMH-1. MD is the distance in feet below the top of the casing, measured along the borehole trajectory, and TVD is the true vertical depth from the same reference point.

\begin{tabular}{lll} 
Description & MD, ft. & TVD, ft. \\
Top of whipstock & 104.70 & 104.7 \\
Top of window & 105.70 & 105.7 \\
Kick-off point & 115.66 & 115.7 \\
Top of broken drill string & $119 \pm$ & $119 \pm$ \\
Top of inflatable casing packer & 34.45 & 34.5 \\
Bottom of packer & 48.92 & 48.9 \\
PZ tube 1 & 20.83 & 20.8 \\
PZ ube 2 & 120.75 & 120.8 \\
Water Table & 132.5 & $132 \pm$ \\
Bottom of blank 2 3/8 in. tubing & 170.69 & 150.6 \\
First pair of 1/8n drilled perforations & $176 \pm$ & 150.6 \\
PZ tube'3 & 180.67 & 150.7 \\
PZ ube 4 & 240.67 & 151.4 \\
Excessive drilling fluid loss zone & $283 \pm$ & $155 \pm$ \\
PZ tube 5 & 298.58 & 157.05 \\
PZ tube 6 & 360.50 & 166.93 \\
Bottom of screen & 480.40 & 176.22 \\
Last pair of 1/8" drilled perforations & $475 \pm$ & $176 \pm$ \\
\hline
\end{tabular}


Table V.2. Completion details for extraction well AMH-2. MD is the distance in feet measured along the well trajectory from the top of the casing.

Description

Top of whipstock:

Top of window:

Kick-off point:

Cement basket \#1:

Cement basket \#2:

Centralizer:

Bottom of blank $41 / 2^{n}$ casing:

Top of slotted screen section:

PZ ube 1:

PZ wbe 2:

PZ tube 3:

PZ tube 4:

PZ tube 5:

PZ ube 6:

Bottom of screen:

Bottom of float shoe:

Bottom of drive pipe guide:
MD

14.04

25.00

11.40

12.40

21.12

21.45

22.2

58.5

98.7

138.8

179.0

237.72

In order to model the performance of the ISAS project, it is necessary to measure, assume, or infer the variation, with distance along the wellbore, of the flux into or out of the formation. Since no flow profile measurements were made for the ISAS test, modelers have been forced to assume that the flux is uniformly distributed over the entire screened or perforated interval. Given the heterogeneous nature of the interbedded sands and clays, and the difficulties encountered in drilling and completing these wells, this assumption seems almost certainly incorrect. The completion details have been summarized here for reference by subsequent investigators, in hopes that it can be combined with some of the indirect evidence bearing on the injection and extraction flux distributions, in order to begin assessing how serious the error might be.

Many difficulties were encountered in drilling and completing the injection well which may have caused it to have an extremely non-uniform flux along its trajectory.

The drill string became stuck after reaming the hole to its final size, when circulation was stopped for one hour while running a final single-shot survey, and ultimately the string was broken off in the hole. In order to salvage the hole, it was completed by washing down a 2-3/8" string of EUE tubing on top of the stuck drill pipe.

As noted in Table V.1, there is a blank string of tubing from the surface to $170.7^{\prime}$, with a perforated string from that point to a measured depth of about 475', and an external packer is set in 8.5/8" casing at about 49'. The first perforations should be at a measured distance of about 176'. The annulus is open, except for drilling debris, etc., from the base of the packer to TD. The section from the base of the packer to about the top of the window should be isolated from the formation by the 8-5/8" casing. However, depending upon how much of the grout was drilled out of the window, the annulus could be open to the formation from the sop of the window at 106' \pm TVD to the first perforation at around 150.6' TVD (176' MD), and 
there is nothing other than debris to prevent air from leaving the tubing at the first set of perforations, flowing up the tubing/borehole annulus, and exiting at any reasonably permeable interval.

Note that the first perforations are around 18 feet below the water table, and it would require an air pressure of only 8.1 psig. to overcome the hydrostatic pressure and allow air to flow from the perforations to the vadose zone.

In Looney et. al. (1991b), pressures are reported for the piezometer ubes at various times during the injection into AMH-1 at 65 SCFM, !70 SCFM, and 270 SCFM. These may indicate periodic plugging of perforations, especially toward the end of the borehole. A careful examination of these data could provide a semi-quantitative estimate of the flux distribution, but this was only partially completed and will not be discussed further in this report.

\section{CONTAMmant CONCENTRATIONS}

Figures V.1 and V.2 show the reported concentrations of TCE and PCE, respectively, from different sampling points in the extraction well, AMH-2, as functions of calendar time. The "continuous" curves are measurements at the surface ( $0 \mathrm{ft}$. along the wellbore), and the depths for values shown as individual points are given in the legend. The sample point closest to the well head is at 22.2 feet along the well trajectory, and the most distant is at $138.8 \mathrm{ft}$. The maximum concentration plotted is $500 \mathrm{ppm}$ by volume; a few higher values were left off to obtain more detail over the later time period.

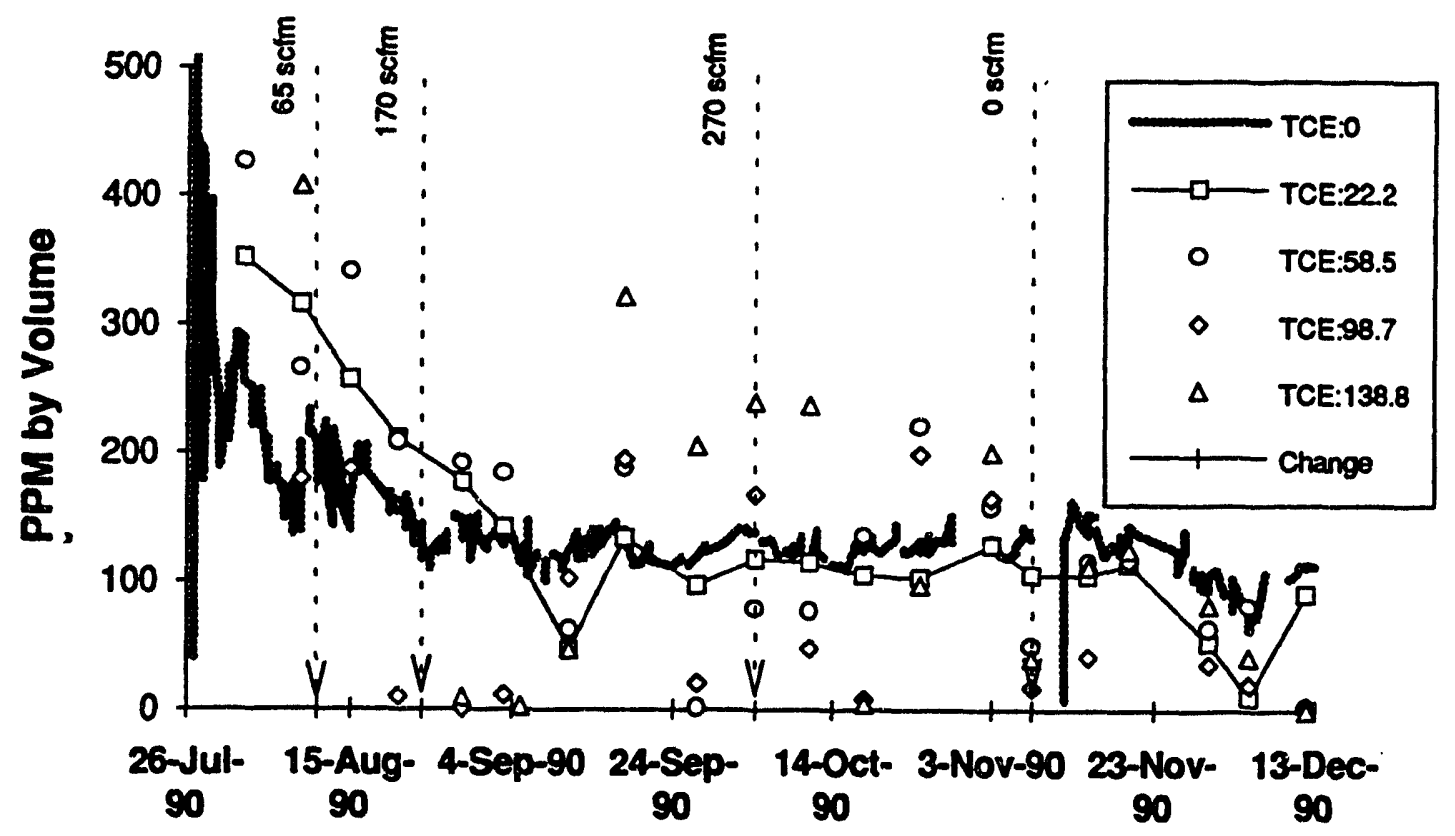

\section{Figure V.1. TCE concentrations measured at the surface and at various sampling points along the wellbore trajectory of AMH-2.}

Note that, although there is considerable scatter, the individual sample tube measurements agree pretty well with the wellhead measurements. The points for AMH-2:22.2 are connected with a line so they can be more easily compared with the wellhead measurements. The dashed arrows were added to show the operating changes, with the first arrow representing the start of injection at 65 SCFM, the second when injection was increased to 170 SCFM, the third at the time it increased to 270 SCFM, and the last when injection was terminated while continuing to operate the extraction well for about six more weeks. There is no visible effect from injecting air on the concentration of TCE measured, with the possible exception of the last period (after air injection ended), when there seems to be a clear decreasing trend in TCE concentration. 
The general impression obtained from examining this plot is that concentrations in the sample tubes probably average higher than the wellhead concentration, and that there is not a clear-cut trend along the wellbore. There is some indication that concentrations in the more distant tubes have a higher average concentration than those closer to the wellhead, possibly indicating that relatively more of the TCE is coming from deeper strata than from the shallower zones - i.e., air entering at points closer to the surface is less contaminated than air from deeper zones.

Fig. V.2 is a similar plot for PCE, which has a very different appearance. As noted previously, the PCE concentration first drops very rapidly, actually dropping below the TCE concentration for several days, then rises more or less coincidentally with the beginning of air injection. Close examination of Fig. V.2 reveals that the association between air injection and the increase in PCE concentration may be only apparent. The increase in PCE concentration actually appears to have started several days before the start of air injection. Furthermore, the concentration did not vary much between early September and midNovember.

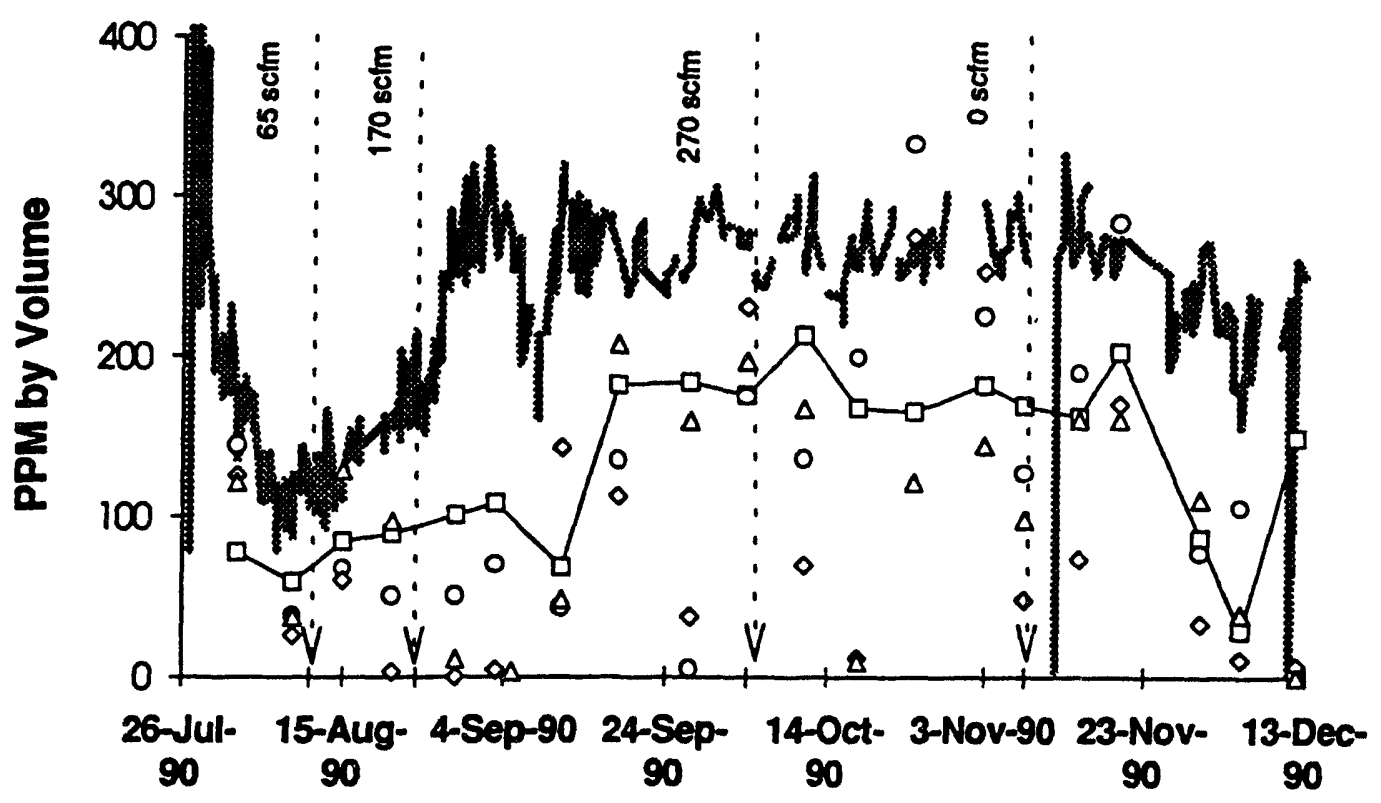

Figure V.2. PCE concentrations measured at the surface and at various sampling points along the wellbore trajectory of AMH-2. See Fig. V.1 for the legend.

Another very curious observation from Fig. V.2 is that the PCE concentrations measured at the surface (upon which the estimates of VOC removal were based) exceed the reported downhole concentrations by a factor of 1.5 to 2 . Overall, the downhole measurements are reasonably consistent with each other, indicating perhaps that PCE is entering the wellbore somewhere past the last point reported (at 138.8 feet), with little additional entry until some point above the sample tube at 22.2 feet. If this is correct, then a substantial fraction of the PCE produced is entering the extracted vapor stream at depths shallower than 22.2 feet, which is difficult to reconcile with the completion records. Of course, there could simply be a substantial measurement error. Is it possible that different techniques or personnel were used for analyzing the wellhead samples and the downhole samples?

\section{Core SAMPle Permeabilities}

In July 1992, we received a copy of the M Area Post Test Characterization Geotechnical Testing Report, O'Brien \& Gere Engineers, Inc., September 16, 1991. This report contains the most complete set of data 
yet obtained on the properties of the sediments within the stratigraphic interval of interest, including measurements of vertical permeability on 18 samples and of horizontal permeabilities on 12 of the samples. These data are reproduced and partially analyzed in the following table.

Table V.3 Permeability Data from Table 2 of O'Brien \& Gere Report.

Lab Borehole No.

1233 MHB-1T

1234 MHB-1T

1235 MHB-1T

1236 MHB-1T

1237 MHB-1T

1238 MHB-1T

1239 MHBB-1T

$1240 \mathrm{MHB}-4 \mathrm{~T}$

1241 MHB-4T

$1242 \mathrm{MHB}-4 \mathrm{~T}$

1243 MHB-4T

1244 MHB-4T

1245 MHB-8T

1246 MHB-8T

1247 MHT-10B

1248 MHT-11C

1249 MHT-11C

1250 MHT- $12 \mathrm{C}$

Average

Standard Deviations

$S$

Sigma

Log normal Approx.
Depth

Class Kv (cm/sec)

$\mathrm{Kh}(\mathrm{cm} / \mathrm{sec}) \mathrm{Kv} / \mathrm{Kh}$

$\operatorname{Ln}(K v) \quad \operatorname{Ln}(K h)$

\begin{tabular}{|c|c|c|c|c|c|c|}
\hline $31-33$ & CS & $1.80 \mathrm{E}-08$ & $4.50 \mathrm{E}-08$ & 0.4 & -17.8329 & -16.9166 \\
\hline $40-42$ & SFC & $3.50 E-08$ & $4.20 \mathrm{E}-08$ & 0.833333 & -17.1679 & -16.9856 \\
\hline $56-58$ & SS & $3.30 \mathrm{E}-04$ & 7.80E-05 & 4.230769 & -8.01642 & -9.4588 \\
\hline $80-82$ & SS & $1.50 \mathrm{E}-03$ & & & -6.50229 & \\
\hline $100-102$ & SS & $5.50 \mathrm{E}-04$ & & & -7.50559 & \\
\hline $110-112$ & SFC & $4.00 \mathrm{E}-04$ & & & -7.82405 & \\
\hline $130-131.3$ & SS & $1.10 \mathrm{E}-03$ & & & -6.81245 & \\
\hline $40-42$ & SFC & 4.00E-07 & $3.70 E-07$ & 1.081081 & -14.7318 & -14.8098 \\
\hline $60-62$ & CS & $5.60 E-04$ & & & -7.48757 & \\
\hline $70-72$ & CS & $4.00 \mathrm{E}-05$ & $1.90 \mathrm{E}-05$ & 2.105263 & -10.1266 & -10.8711 \\
\hline $80-82$ & SS & $1.40 \mathrm{E}-03$ & & & -6.57128 & \\
\hline $110-112$ & SFC & $6.20 \mathrm{E}-09$ & $1.20 \mathrm{E}-08$ & 0.516667 & -18.8987 & -18.2384 \\
\hline $155-157$ & SS & $3.40 \mathrm{E}-07$ & $3.40 \mathrm{E}-07$ & 1 & -14.8943 & -14.8943 \\
\hline $170-171$ & CS & $4.00 \mathrm{E}-07$ & $5.50 \mathrm{E}-07$ & 0.727273 & -14.7318 & -14.4133 \\
\hline $201-201.7$ & SS & $4.30 \mathrm{E}-05$ & 4.50E-05 & 0.955556 & -10.0543 & -10.0088 \\
\hline $165-167.75$ & $C S$ & $1.90 E-07$ & $5.60 \mathrm{E}-06$ & 0.033929 & -15.4762 & -12.0927 \\
\hline $200-202.4$ & SS & $2.50 \mathrm{E}-04$ & $8.50 \mathrm{E}-05$ & 2.941176 & -8.29405 & -9.37286 \\
\hline \multirow[t]{5}{*}{$200-202.2$} & $\mathbf{S}$ & $3.80 \mathrm{E}-04$ & $120 E-04$ & 3.166667 & -7.87534 & -9.02802 \\
\hline & & $3.64 E-04$ & $2.95 \mathrm{E}-05$ & $1.50 E+00$ & $1.12 E+01$ & $1.31 \mathrm{E}+01$ \\
\hline & & 0.0004789 & 4.23E-05 & 1.306208 & 4.277834 & 3.346676 \\
\hline & & 1.3152543 & 1.4342155 & 0.871207 & & \\
\hline & & 1.0021291 & 1.0570835 & & & \\
\hline Mode & & $9.28 E-05$ & & & $1.52 \mathrm{E}-09$ & 7.63E-09 \\
\hline Median & & 0.0002204 & & & $1.43 E-05$ & $2.06 \mathrm{E}-06$ \\
\hline Mean & & 0.0003641 & 2.95E-05 & & 0.134549 & 0.000558 \\
\hline
\end{tabular}

Table V.4 summarizes a discrete approximation, with four values, of a log-normal distribution for the permeability of the SRIDP subsurface. The mean is the arithmetic average of the 18 vertical permeability measurements. The $\sigma$ value was calculated by using the sample standard deviation to calculate a coefficient of variation, $S$, and the fact that $S=\sqrt{e^{\sigma^{2}}-1}$ for a log-normal distribution. 
The column "Fractions" gives the fraction of the total stratigraphic interval with the corresponding average permeability in the column headed $k$. The last column sums to the value of average permeability given as the mean, which serves as a check on the calculation.

In other words, a system having only the four tabulated permeability values $4.30 \cdot 10^{-5}, 1.03 \cdot 10^{-4}$, $2.27 \cdot 10^{-4}$, and $7.82 \cdot 10^{-4}$, in proportions $0.1111,0.2222,0.3333$, and 0.3334 , respectively, would have exactly the same mean and standard deviation as the original 18 data points. These proportions were selected to agree with the corresponding fractions of mud, sandy mud, muddy sand, and sand from the coarse grid stratigraphic model described in Section VI.

The average permeability is only $0.000364 * 1033=0.376$ darcies, compared to about 50 darcies reported by Looney et al. from an analysis of the transient pressure response in the vertical well SVE test. This suggests that the coarser sediments were not represented in the sample in proportion to their occurrence in the ISAS demonstration area.

Table V.4. Four discrete permeability values with the same mean and standard deviation as the $0^{\prime}$ Brien and Gere data. From lowest to highest $k$, these are identified with mud, sandy mud, muddy sand, and sand, respectively.

$\begin{array}{lllll}\text { mean } k & \text { kbar } & 3.64 \mathrm{E}-04 & & \\ \text { sd of } \ln (\mathrm{k}) & \sigma & 1.002 & & \\ \text { median } k & \mu & 0.000220396 & & \\ & \varepsilon & 0.0000001 & & k \\ \text { Fractions } & \text { Cumill. } & \text { Phinv } & k & \\ 0 & 0 & -5000000 & & \\ 0.1111 & 0.1111 & -1.220698778 & 4.30 \mathrm{E}-05 & 4.78 \mathrm{E}-06 \\ 0.2222 & 0.3333 & -0.430818545 & 1.03 \mathrm{E}-04 & 2.29 \mathrm{E}-05 \\ 0.3333 & 0.6666 & 0.430543423 & 2.27 \mathrm{E}-04 & 7.57 \mathrm{E}-05 \\ 0.3334 & 1 & 5000000 & 7.82 \mathrm{E}-04 & 2.61 \mathrm{E}-04 \\ & & & \text { Sum } & 3.64 \mathrm{E}-04\end{array}$

Another set of values can be derived from the same data by calculating the mean and standard deviation of the natural logarithm of $k$. The results are shown in Table V.5. Note that the arithmetic mean value, calculated from the log mean, log standard deviation, and the mathematical properties of the log-normal distribution, is now $0.135 \mathrm{~cm} / \mathrm{sec}$, or about 140 darcies.

From these two analyses of the same data set, it is obvious that the heterogeneity parameter estimate from actual permeability distribution data is subject to a very large error when only a few samples are available. In the Benton waterflood example of the heterogeneity model approach, Chesaut et. al. reported close agreement between the $\sigma$ value derived from core analyses and the value obtained from a fit of the injection and production data. However, there was a much larger data set available. Of about 200 wells in the field, about 60 were cored, and a sample was taken from each foot of core (approximately 40 to 50 per well) for air permeability and other petrophysical property measurements. 
Table V.5. Four discrete permeability values with the same natural logarithmic mean and standard deviation as the O'Brien and Gere data. From lowest to highest $k$, these are identified with mud, sandy mud, muddy sand, and sand, respectively.

$\begin{array}{lllll}\text { mean } k & \text { kbar } & 1.35 \mathrm{E}-01 & & \\ \text { sd of } \ln (\mathrm{k}) & \sigma & 4.278 & & \\ \text { median } k & \mu & 1.42826 \mathrm{E}-05 & & \\ & \varepsilon & 0.0000001 & & \\ \text { Fractions } & S & \text { Phinv } & k & \\ 0 & 0 & -5000000 & & \\ 0.1111 & 0.1111 & -1.220698778 & 2.32 \mathrm{E}-08 & 2.58 \mathrm{E}-09 \\ 0.2222 & 0.3333 & -0.430818545 & 7.44 \mathrm{E}-07 & 1.65 \mathrm{E}-07 \\ 0.3333 & 0.6666 & 0.430543423 & 2.36 \mathrm{E}-05 & 7.86 \mathrm{E}-06 \\ 0.3334 & 1 & 5000000 & 4.04 \mathrm{E}-01 & 1.35 \mathrm{E}-01 \\ & & & \text { Sum } & 1.35 \mathrm{E}-01\end{array}$

In summary, attempting to represent these data by a log-normal distribution teads to an average value that is perhaps a factor of three 100 large or a factor of more than one hundred 100 small, and the $\sigma$ value ranges from about 1 to more than 4 . This illustrates a range of uncertainty that could be reduced by obtaining more data, provided that the heterogeneity parameter model is conceptually valid.

\section{Pressure Response: Vertical Well VacuUm Extraction Test}

Looney et. al. (1991a) reported on the short-lerm vacuum extraction (VE) test summarized briefly in the Section I. Although the sequence and duration of applying vacuum to different well screens was not planned for analysis as a series of transient pressure tests, some of the data can be used to obtain estimates of the air permeability in the vicinity of well VB-1, the principal extraction well for the test.

In their analysis, Looney et al. (1991a) used the pressure response data for screens VB-2A and VB-3B, during the first period that vacuum was applied to VB-1 only, to estimate the permeability and specific storage for an interval 70 feet thick more or less centered between the ground surface and the water table. As they pointed out, Massman (1989) showed that groundwater models can, under some conditions, be used to model air flow in the vadose zone.

The conceptual model they used is a leaky aquifer system, with the pumped interval partially isolated from the atmosphere by a continuous clay layer above the screened interval. The water table provides the lower no-flow boundary. Except for the fact that air is the mobile phase instead of water, the system is treated as a classical leaky aquifer test, with the "pumped aquifer," i.e., the interval to which vacuum is assumed to be applied at VB-1 (the "pumped well"), treated as a confined "aquifer" separated from a shallower "aquifer" by a 2-foot "aquitard." In the Hantush and Jacob leaky aquifer model, this shallower aquifer is assumed to be a constant head region-i.e., not enough fluid flows from the shallow, leaking aquifer to change its pressure. In the present application, the constant head region is the atmosphere. With these assumptions, and the analytical solution of Hantush and Jacob, Looney et al. calculated an air permeability of 42 darcies for the "pumped aquifer" and a permeability of 0.05 darcies for the "aquitard." Because of the flow geometry, the value of 42 darcies is an estimate of the horizontal permeability of the sandy intervals, while 0.05 darcies might represent a reasonable estimate for the vertical permeability, at least for the upper 30 feet or so. 
This analysis may be slightly in error. Using flow equations for water to interpret results for pressure transients induced by the extraction of gases from porous media is common practice among petroleum reservoir engineers. Mathews and Russell (1967) and later Earlougher (1977) demonstrated that the same basic differential equations can be used to represent the single-phase flow of either gas or water, except that in the case of gas flow at pressures below 100 atmospheres or so, the appropriate dependent variable is the square of the fluid pressure rather than the pressure itself (neglecting gravity). Since, with this transf rrmation of dependent variables, the same differential equations apply, it is obvious that all the analytical results derived for the flow of water can be used to analyze the flow of low-pressure gas for similar sets of boundary conditions.

The problem lies in the violation of some of the conditions necessary for the validity of the Hantush and Jacob solution. The most serious problem is that the pumped well must be open only to the pumped aquifer. This condition is clearly violated in the VE test because VB-1 is open to permeable intervals both above and below the middle 70 feet (the total screen length in this well is $100 \mathrm{ft}$ ). If the intervals above and below this zone are permeable, their pressures, as well as the pressure in the middle zone, will decrease with time so long as extraction continues.

The actual flow geometry and boundary conditions are complex, even if the permeability to air from the surface to the water table were homogeneous and isotropic. However, we show below that the data are reasonably consistent with the line-source solution for a well producing a slightly compressible fluid at constant rate from a radially infinite, isotropic, homogeneous layer of constant thickness.

In the ground water literature, the line-source approximation is known as the Theis solution, and in the petroleum reservoir engineering literature it is known as the Homer solution. An important result of the line-source solution is that $\Delta p$ (for liquid flow) or $\Delta p^{2}$ (for low-pressure gases) varies linearly with the logarithm of time for the "infinite-acting" time period.

At early time, a plot of $\Delta p$ (or $\Delta p^{2}$ ) vs. $\log (t)$ will not be linear because of well bore storage effects and the approximation of the actual mathematical solution by its asymptotic form. Well bore storage effects can be recognized by plotting $\log (\Delta p)$ vs. $\log (t)$. A slope of unity indicates that the pressure behavior is completely dominated by well-bore storage, and hence cannot be used to determine properties of the porous media within which the well is completed.

At late time, the effect of lateral boundaries (usually assumed to be either closed or held at constantpressure) affects the shape of the plot. If the system has a closed outer boundary, $\Delta p$ (or $\Delta p^{2}$ ) becomes a linear function of $t$ rather than of $\log (t)$, and if it has a constant-pressure outer boundary, $\Delta p$ (or $\Delta p^{2}$ ) reaches a constant value independent of $t$.

The latter behavior is characteristic of true steady-state flow, and a plot of $\Delta p$ (or $\left.\Delta p^{2}\right)$ vs. $t$ will have the same appearance as the leaky-aquifer type curves of Hantush and Jacob-i.e., a progressive flattening of slope on a semi-log plot until it reaches a constant value. In the case of a lateral constant-pressure boundary, the fluid source required to maintain constant mass within the system (and hence a steady-state pressure distribution) is located at some fixed radial distance from the pumping well. For the leaky-aquifer model, the fluid source is distributed above or below the pumped aquifer and is assumed to supply fluid at a rate proportional to the local difference in pressure between the active and the passive aquifers.

In analyzing the vertical well data, we first prepared a plot (Fig. V.3) of $\log \left(\Delta p^{2}\right)$ vs. $\log (t)$ for screens VB-2A and VB-3B, using the data given by Looney $e t$ al. (1991a). The data reported for all screens were converted to absolute pressures, using the reported barometric pressure, and used to calculate values of $\Delta p^{2}$. The first two points for VB-2A could be connected by a straight line with a slope close to unity. However, it appears that wellbore storage effects are not important after the first two or three data moints.

Figure V.4 shows the plots of $\Delta p^{2}$ vs. $\log (t)$. Note that the data for both response wells are reasonably well represented by straight lines after about 1 hour of drawdown in VB-1, and that they show no tendency to flatten, as would be required for either a leaky-aquifer model or a constant pressure lateral boundary. Also shown are lines fitted by least-squares to the "late-time" data for each response well. Table 


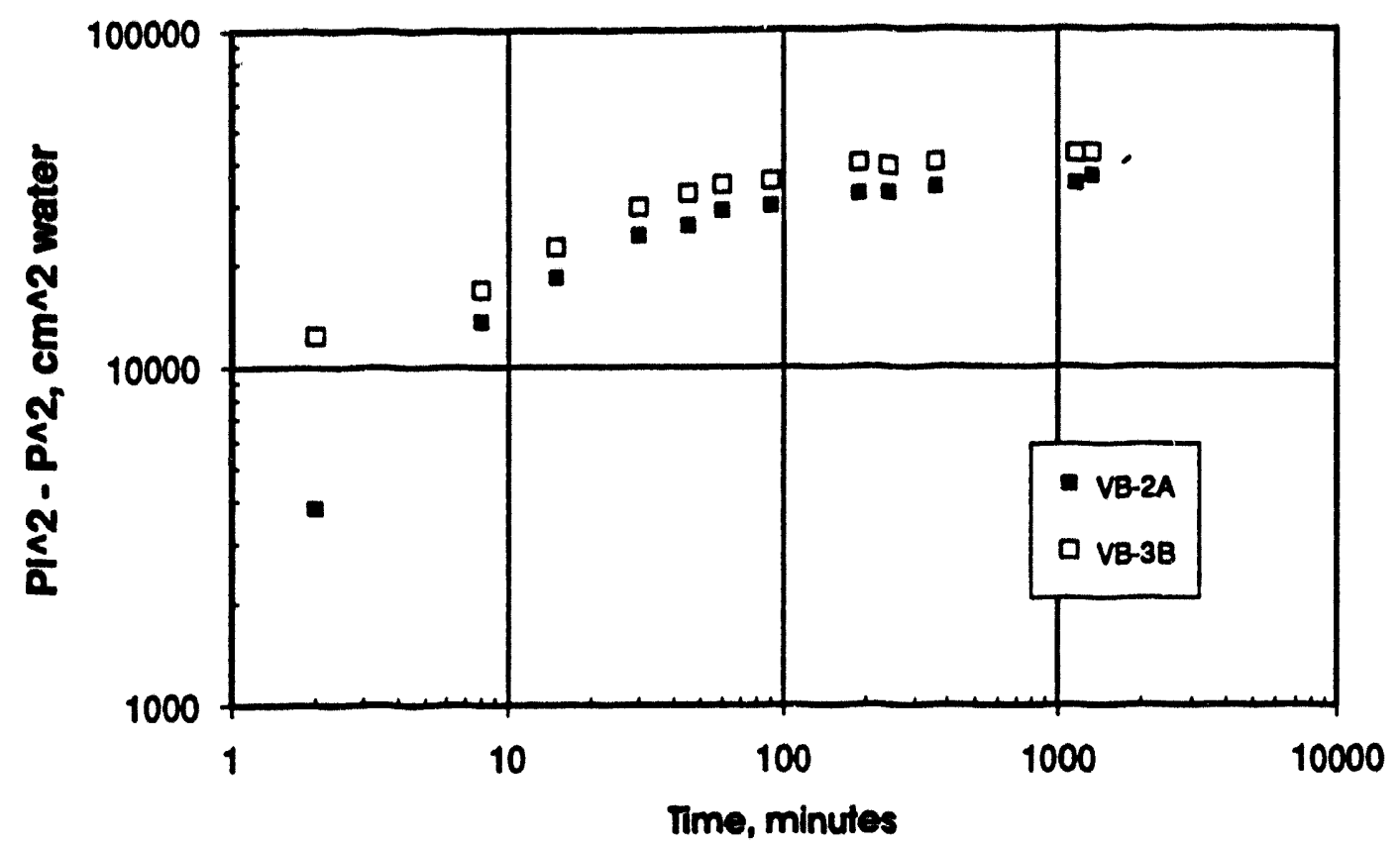

Figure V.3. Log-log plot of the squared pressure response in VB-2A and VB-3B to extraction from VB-1

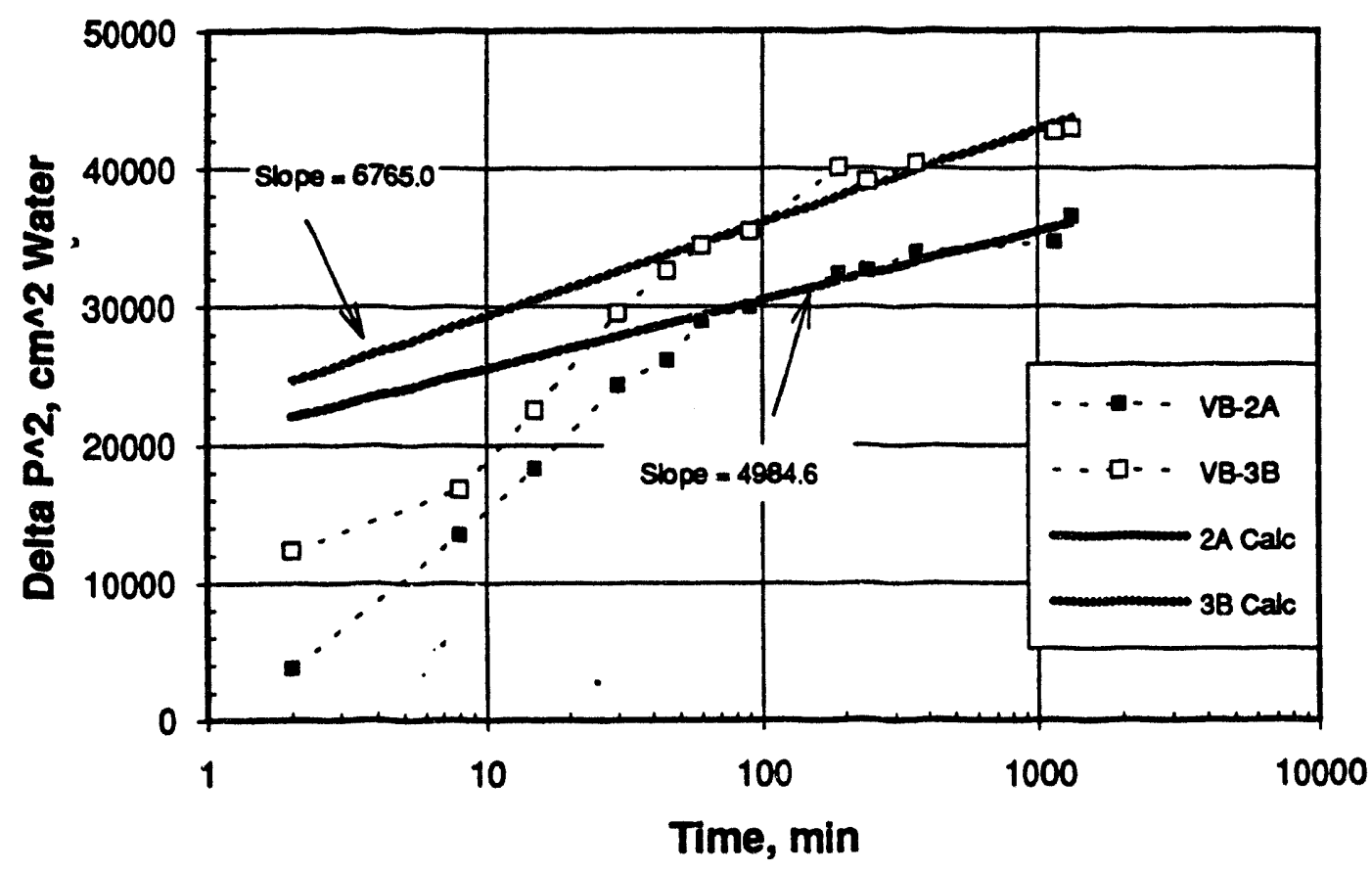

Figure V.4. Theis (or Horner) plots of the squared pressure response in VB-2A and VB-3B to extraction from VB-1. 
V.6, in addition to summarizing the data used, also shows the result of using the Excel function LINEST to determine the least-squares lines. From the plots in Fig. V.4, it can be seen that the line-source solution is applicable, and the slopes of the least-square lines can be used to estimate the permeability.

The data from both response wells are combined on Fig. V.5. According to the line-source solution, the pressure response should be a function of $\log \left(t / r^{2}\right)$, where $t$ is the time and $r$ is the distance from the active well to the responding well. There is considerable scatter, but the data can be fit reasonably well by the least-squares straight line determined by using LINEST, which is also plotted on Fig V.5. .

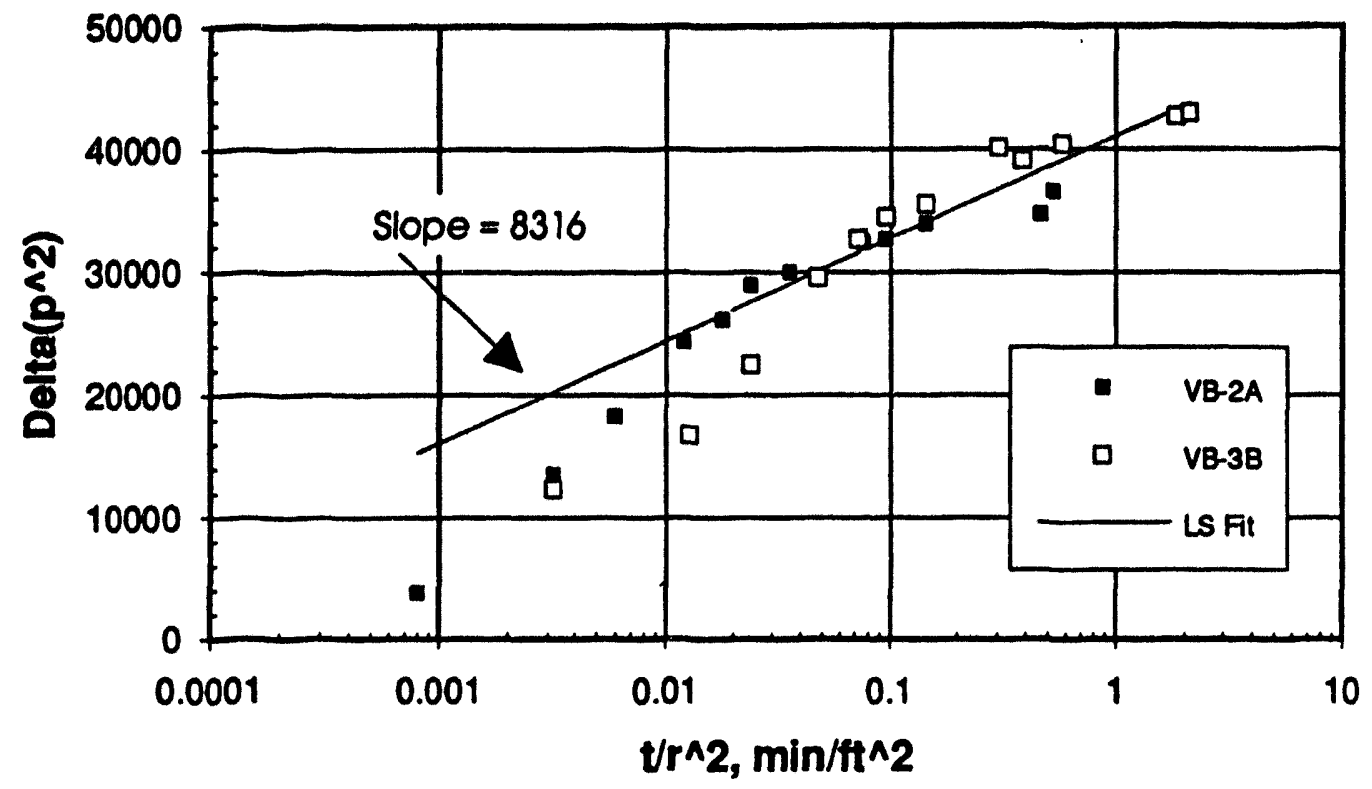

Figure V.5 Theis plot for combined data from screens VB-2A and VB-3B, assuming radial flow geometry.

In fitting these data, some of the early time points were excluded from the least-squares analysis. Several choices were used in each data set for the smallest value of $t\left(\right.$ or $\left.t / r^{2}\right)$ included in the analysis, and the final choice used was determined by selecting the choice which resulted in the largest value for the regression coefficient and the smallest standard error of estimate for the slope. Table V.6 summarizes these caiculations. The lines in bold-face italics show the values used to estimate permeabilities.

The rationale for using $\Delta p^{2}$ is given by Earlougher (1977) in discussing the application of the following equation:

$$
p_{\alpha f}^{2}=p_{\mathrm{i}}^{2}-50,300 \cdot\left(z_{i} m_{g i}\right) \cdot\left(\frac{p_{x}}{T_{x c}}\right) \cdot\left(\frac{q T}{k H}\right) \cdot\left\{\mathrm{P}_{\mathrm{D}}\left(t_{D}\right)+S+D \cdot|q|\right\}
$$


Table V.6. Least-Squares Analyses of Transient Pressure Data from the VE Test. The first column is the well designation, the second is the minimum value of the independent variable included in the least-squares fit, the third is the resulting regression coefficient, the fourth is the slope, and the last is the standard error of estimate for the slope. Units of the slope are $\mathrm{cm}^{2}$ of water per $\log$ cycle.

\begin{tabular}{|lllll|}
\hline Well & $\mathbf{X}_{\text {min }}$ & $\mathbf{R}^{2}$ & Slope, $\mathbf{m}$ & $\mathbf{S}_{\mathrm{e}}(\mathbf{m})$ \\
\hline VB-2A & 45 & 0.913 & 5843 & 739 \\
& 60 & 0.940 & 4985 & 566 \\
& 90 & 0.912 & 4658 & 721 \\
VB-3B & 30 & & & \\
& 45 & 0.922 & 7613 & 831 \\
& 60 & 0.931 & 6765 & 753 \\
& 90 & 0.920 & 6183 & 813 \\
& & 0.886 & 5637 & 1009 \\
Both & 0.0128 & 0.841 & 9432 & \\
& 0.018 & 0.868 & 8316 & 894 \\
& 0.024 & 0.849 & 8248 & 899 \\
& 0.036 & 0.857 & 7352 & 831 \\
& 0.048 & 0.834 & 7330 & 943 \\
& 0.072 & 0.805 & 6940 & 1030 \\
\hline
\end{tabular}

In Eq. (V.1), the following notation is used:

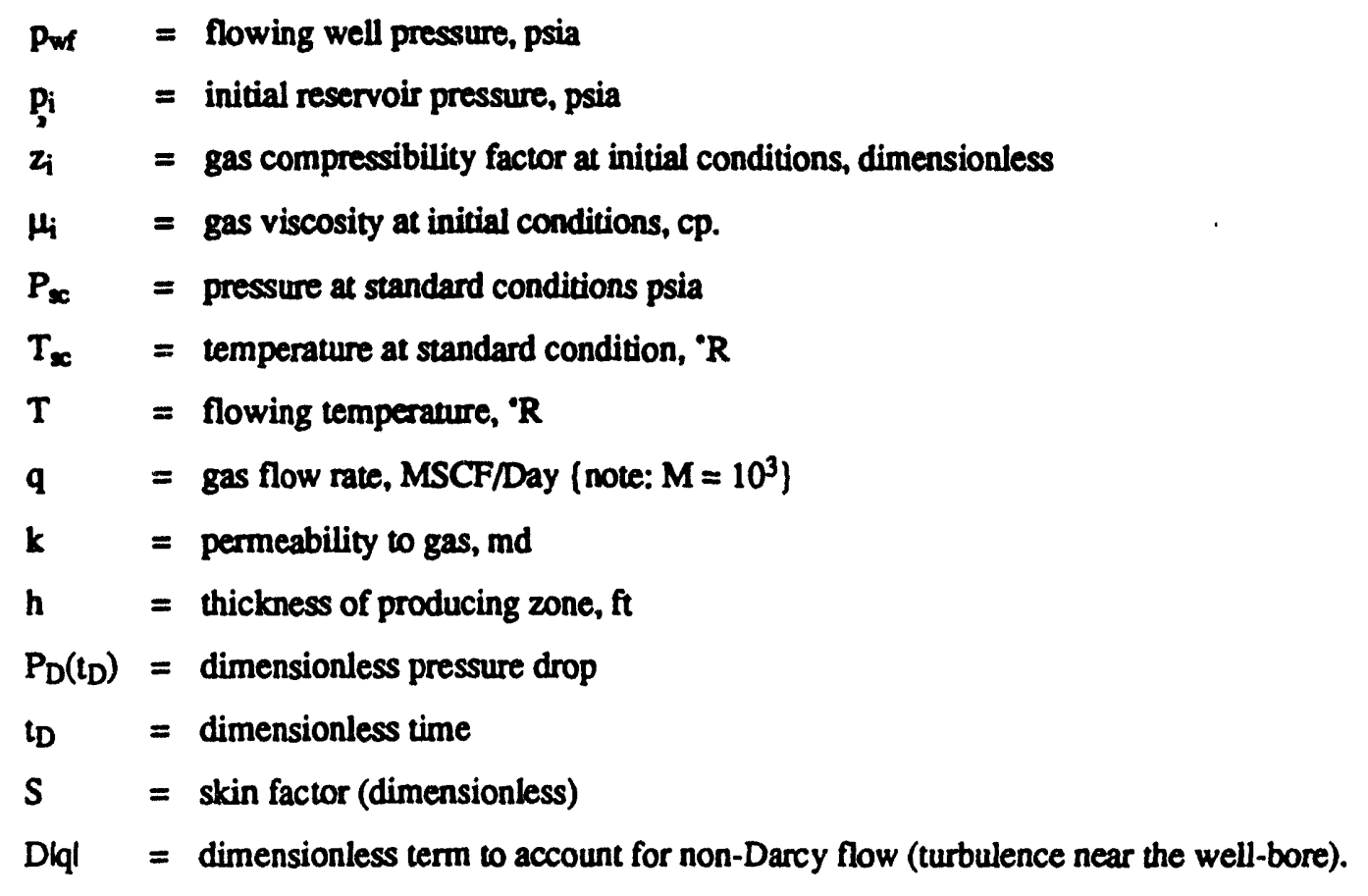

Earlougher states that Eq. (V.1) generally applies for $p<2000$ psig. 
Equation (V.1) is written for pressure measured at the flowing well. For the VE test, we have measurements at two observation wells instead. This merely requires replacing $P_{D}\left(t_{D}\right)$ by $P_{D}\left(t_{D}, r_{D}\right)$, since $P_{D}\left(t_{D}\right)$ is just Earlougher' 3 abbreviation for $P_{D}\left(t_{D}, 1\right)$, and $r_{D}=1$ at the operating well. Earlougher also shows that the following approximation is valid for dimensionless time greater than about 100:

$$
p_{d}^{2}=p_{i}^{2}-50,300 \cdot\left(z_{i} m_{s i}\right) \cdot\left(\frac{p_{\alpha}}{T_{\alpha}}\right) \cdot\left(\frac{q T}{k H}\right) \cdot\left\{1,151 \cdot \log \left(\frac{t_{d}}{r_{d}^{2}}\right)+0.405\right\}
$$

Substitution of the following values of the parameters (applicable to conditions during the test) into Eq. (V.2), which is of the form $\Delta P^{2}=m \log t+$ constant,

$$
\begin{aligned}
& T_{x}=520^{\circ} R\left(60^{\circ} \mathrm{F}\right) \\
& T \cong 515^{\circ} \mathrm{R}\left(55^{\circ} \mathrm{F}\right) \text { from horizontal well test data } \\
& \mathrm{P}_{\boldsymbol{x}}=14.696 \mathrm{psia} \\
& \mathrm{z}_{\mathrm{i}} \cong 1.0 \text { (ideal gas) } \\
& H_{8 i} \cong \quad 0.0178 \text { air @ } 55^{\circ} \mathrm{F}, \text { Earlougher }(1977) \\
& \mathrm{h} \cong 121^{\prime}-23^{\prime}=98^{\prime} \text { top filter to WT in VB-1, Looney (1991a) }
\end{aligned}
$$

results in

$$
k=1531 \cdot \frac{q}{m},
$$

where the slope, $m$, must be expressed in $\mathrm{psi}^{2}$ per $\log _{10}$ cycle. The rate, $q$, is not given precisely, but is stated as " 400 to 500 SCFM" in Looney et al. (1991a); we used 450 SCFM.

From the slope values given in Table V.6 (bold face), the permeability estimates are:

- VB-2A 98 darcies

- VB-3A 72 darcies

- Both 59 darcies

These results agree quite well with each other and with the estimate by Looney et al. (1991a) of 42 darcies for the horizontal permeability. However, since there is no sign of the flattening in slope at late time indicating leaky aquifer behavior, the estimate for vertical permeability obtained by Looney et al. (1991a) probably has no physical significance.

\section{TRACER TEST}

We modified the heterogeneity parameter model for analyzing the helium tracer test. However, this analysis was not completed due to funding constraints and, accordingly, we defer the documentation of this work indefinitely. 


\section{GeOLOGIC AND GEOPHYSICAL ANALYSES}

\section{STRATIGRAPHIC CORRELATIONS}

An accurate three-dimensional stratigraphic model is required for computer simulations of the hydrologic and contaminant transport response to the remediation processes applied at the Savannah River In-Situ Air Stripping Demonstration Site (ISASDS). The stratigraphic model provides the spatial distribution of lithologic units, to which can then be assigned porosity, fluid saturations, permeability, and contaminant concentrations by various methods.

The initial interpretation of the subsurface stratigraphy made by Savannah River Laboratory staff and tireir contractors was a simple layered cross section through wells MHT1c, MHT2c, MHT4c, MHT6c, MHT\&c, and MHT1OC. The stratigraphic units, of more or less uniform thickness, were defined primarily on the basis of four clay layers encountered in the wells. There was no definition of the complex interbedding of sand and clay lithologies between these major clay units. Based on our review of the lithologic descriptions of the core from all ten of the cored wells, we believe that the subsurface section is much more heterogeneous than portrayed by the SRL geologists.

Since heterogeneity profoundly affects all aspects of remediation process performance, from initial contaminant distribution to the efficiency with which contaminant is removed. and important part of our analysis and evaluation task was to define more accurately the heterogeneity of the sedimentary section beneath the ISASDS. The resulting detailed stratigraphic framework provides a basis for more accurately predicting the movement of VOCs in the subsurface.

\section{Approach}

None of the LLNL/LBL Analysis and Evaluation Team were present during collection of core from the wells at the ISASDS, nor were we able to review the core during our investigations. SRL ISAS project staff and management provided lithologic data to LLNL, along with selected geophysical logs from the ten MHT wells. We assume that the data were properly generated and checked for integrity. Even so, some lingering questions remain regarding quality of the data.

The first lithologic data provided to LLNL were brief core descriptions generated at the rig by the drill site geologists. Several different geologists described the core, resulting in considerable variation in the amount of detail and, apparently, the terminology used for different sediments. This made it very difficult to make stratigraphic correlations, and thus these data were of only limited use. This may be the reason for the very simple initial cross-section prepared by SRL personnel.

A second set of lithologic data (in ASCIn files on diskettes) was subsequently furnished to LLNL by SRL. These data were generated in the laboratory, using a binocular microscope to examine the core. Each foot of core was described in detail by a geologist, and the percentages of gravel, sand, and mud were estimated. Using these data, they assigned a lithologic name to each foot of core. A number of other parameters were recorded for each foot of core:

$\begin{array}{ll}\text { - } & \text { Depth } \\ \text { - } & \text { Core recovery } \\ \text { - } & \text { Lithologic classification } \\ \text { - } & \text { Induration } \\ \text { - } & \text { Color } \\ \text { - } & \text { Sedimentary structures }\end{array}$




- $\quad$ \% gravel (>2 mm)
- $\quad$ \% sand (62.5 microns-2 mm)
- $\quad$ maxd (<62.5 microns)
- $\quad$ Most heavy minerals
- $\quad$ Average roundness of quartz grains
- $\quad$ Carbonate fraction
- $\quad$ Sorting
- $\quad$ Porosity
- $\quad$ Pore type
- muscovite
\% lignite
\% sulfides

This more comprehensive and detailed data set allowed us to develop improved correlations between wells, which show a more typical interfingering of clays and sands than were portrayed by the simple "layer cake" model.

Unfortunately, the lithologic descriptions resulting from the detailed petrographic microscope examination (the second data set) did not correlate very well with the data from the well-site examination. For example, a thick clay layer was described in MHT1c between 36-42' depth (near 325' elevation shown on the right-hand side of Fig. VI.2) by the well-site geologist. This clay was not identified in the laboratory examination of the core (shown on the left-hand side of Fig. VI.2). Interestingly, the high gamma response and low resistivity recorded on the geophysical logs at this depth interval are typical of clay layers. The drill site geologist may have interpreted a clay layer from the logs, without confirmation from the core, although this is unlikely, since there is a description of clay in the lithology log. These discrepancies are disturbing, but without performing our own review of the core samples, we had to decide arbitrarily to accept one of the two data sets. Since the laboratory examinations were done with less time pressure than the well-site examinations, we chose to use only the lithology from the lab analyses in our interpretation of the stratigraphy. Figures VI.2-VI.11 show the comparison of lithology generated by the drill site geologist and the lab geologist for wells MHTIc through MHT10c, respectively.

We classified the lithology of the sediments based on Folk (1968). The temary plot in Fig. VI.1 shows the classification and nomenclature scheme. The lithologic names are based on the relative percentages of the gravel, sand, and mud grain size fractions. SRL geologists used a different classification scheme summarized in Table VI.1. 


\section{Table VI.1. Lithologic classifications used by Savannah River Laboratory at the ISAS demonstration site.}

\begin{tabular}{|l|l|}
\hline Classification & Criterion \\
\hline Pebbly & (>25\% gravel) \\
\hline Sand & (<20\% mud) \\
\hline Clayey sand & (20-50\% mud) \\
\hline Sandy clay & (50-80\% mud) \\
\hline Clay & (>20\% mud) \\
\hline
\end{tabular}

The basic difference in these two classification schemes is in the cutoff percentages for the various size fractions. Folk uses the ratio of sand and mud to define the lithologic name, with sediments containing $>5 \%$ gravel termed "gravely". SRL staff also use the term "clay". This is imprecise, since the estimated fine fraction is $<62.5$ microns diameter, which includes both clay and silt. "Mud" is the correct term for describing these data. Using Folk's classification scheme, we assigned lithologic names, at one foot intervals, to the entire stratigraphic section penetrated by each of the wells.

Geophysical logs were also provided with the lithologic data. Of all of the logs, the natural gamma $\log$ is the most useful for stratigraphic correlations. The natural gamma tool detects the amount of gamma radiation emitted by the sediments, which is a function of the detrital and secondary mineralogy. There is no indication of artificial radioactive contamination in the logs.

There is generally good correlation of gamma signawures between the boreholes, reflecting a degree of lateral continuity of the beds. This is encouraging, except that the highs and lows of the gamma traces do not always correlate with lithology. Fine-grained sediments generally correlate with relatively high gamma radiation, while the sands and gravels typically emit bower levels of natural radiation. This relationship has been well documented in many places. Unfortunately, in the ISASDS data, we observe many inconsistencies in this classical correlation of lithology and geophysical log response. There are numerous examples where the high gamma peaks are not associated with corresponding clay units. This may be the result of poor sampling and/or inconsistencies in describing the sediments. This could also result from variation in the mineralogical content of the sediments. Certain radioactive minerals may be present in some of the sands and gravels, giving the relatively "clean" clastic deposits a "clay-type" signature.

\section{Data interpretation}

The available sedimentary data from the ISASDS allow for only a basic lithostratigraphic framework construction at this time. Apparently, very little data have been generated on the formal stratigraphy of the subsurface at ISASDS. Formal stratigraphic units should be identified to complete the evaluation of the stratigraphy, since the formal stratigraphic relationships will support the current lithostratigraphic correlations in this report.

We used a CADD program to construct the stratigraphic sections for each well. The lithology was divided into 4 generic groups: sand, muddy sand, sandy mud, and mud. This list represents depositional environments of decreasing energy conditions. Stratigraphic data were keyed in for each well, and a stratigraphic column was constructed. Digital geophysical log data were plotted alongside the lithologic section for comparison and correlation purposes. These individual plots are a useful display of the stratigraphy for each well (Figs. VI.2-VI.11).

Next, the stratigraphic sections were used to construct cross sections through the study area. Two cross sections were created: A-A' through MHT1c, MHT2c, MHT4c, MHT6c, MHT\&c, MHT10c and B-B' through MHT3c, MHTSc, MHT7c, MHTYc. These lines of sections are generally sub parallel, and trend 
southeast-northwest (Fig. VI.12). Stratigraphic correlations are based simply on depth and lithology. In making the correlations, we attempted to honor the character of the gamma log as much as possible, since it should best reflect the lithology and mineralogy of the sediments. The good agreement of the gamma logs provides support for the lithologic correlations. Unfortunately, there are no other independent criteria to support the correlation of the beds, such as could be provided by a review of the core itself.

The cross sections (Figs. VI.13 and V.14) portray a shallow marine depositional environment characterized by thinly bedded to massive fine-grained clastic sediments. The finest-grained sediments, the muds and sandy muds, display some amount of lateral continuity, but are not as continuous as the sands. Facies changes are rapid; the muds are generally lenticular deposits, laterally grading into the coarser-grained sands.

The lateral continuity of these fine-grained sediments is key to the modeling of the hydrology of this area, since the muds are the most impermeable of the sediments in the subsurface, acting as barriers to ground water flow below the water table, to water and VOCs percolating downward from the surface, and to air flow from the surface or from the injection well to the extraction well in the ISAS demonstration. Our ability to correlate these thin beds is basically limited to a simple depth correlation in each hole, while honoring the character of the gamma logs. In cases where the muds terminate between wells, we can only guess where the lithology changes.

Another consideration is that the stratigraphic framework is a three dimensional problem, and our confidence in stratigraphic relationships decreases with increasing distance from the line of section. We have attempted to pull together the stratigraphy of the two lines of section (Figs. VI.15 and VI.16). In this process, we correlated the major lithostratigraphic units between the two lines of section. Using the elevation and thickness data from each of the. 10 wells, we modeled the upper and lower bounding surfaces of each unit. The result is a series of structural contour maps and isopach maps of each unit. Cross sections are then cut through these surfaces, providing a view of the lateral continuity of each unit. This has been done for most of the fine-grained units, although some of the thin, isolated muddy beds are too small to model.

This stratigraphy is typical of a near shore, shallow marine depositional environment, characterized by periodic marine transgressions and regressions. The lenticular nature of these fine-grained muds is a result of this continuing change in base level and corresponding energy conditions.

\section{Coarse Grid for Hydrologic Modeling}

The cross sections given in Figs. VI.13 and VI. 14 are not directly usable for simulation of subsurface flow and transport, because we have no simple mechanism of assigning flow properties at this level of detail. In order to provide a more useful representation for hydrologic modeling, we developed a method for summarizing the lithostratigraphy numerically at a spatial scale consistent with a practical grid spacing. We constructed a grid composed of $1 \mathrm{~m}$ (vertical) by $10 \mathrm{~m}$ (horizontal) rectangular grid blocks that could be superimposed on the cross sections (Figs. VI.17 and VI.18). This was done with the CADD system. The grid was constructed and then overlaid on the computerized image of the cross section. A unique lithology was assigned to each rectangular gridlock, depending on the dominant lithology enclosed by the grid block. Permeabilities were then assigned to each lithology according to several methods.

Grain size data were provided to LLNL by SRL. Grain size distribution analyses were performed on closely-spaced core samples from the following wells: MHTIc, MHTSc, MHT7c, and MHT9c. From these sieve analyses, several statistical parameters were calculated, such as standard deviation, skewness, kurtosis, and median. An additional sorting parameter was also calculated.

We used these data and the formula derived by Krumbein and Monk (1942) to estimate permeabilities of the sediments. The functional relationship of grain size distribution and permeability is given below:

$$
k=760 \cdot G M^{2} \cdot e^{-131 \cdot s}
$$

where $k=$ coefficient of permeability, $G M=$ geometric mean distribution in millimeters, and $s=$ phi standard deviation. 


\section{Problems encountered and suggestions for future work}

It has been frustrating working with data generated by another institution. LLNL was not involved with the initial development of the project, thus we had no control of the procedures used to collect and describe the data. Nor did we have input on quality control and peer review of the data and interpretations.

1) The initial lithology descriptions should be made by the drill site geologist. His job is to assure quality control of the core as it is extracted from the borehole. A brief, yet detailed description should be made of the samples at this time, and stratigraphic contacts should be assigned as accurately as possible. A set of criteria should be established for all geologists to follow that are involved in the project. This will result in consistent litbologic descriptions. Standard rock or soil color charts should be used. This procedure was not followed during the drilling of the 10 wells at the SRS, as evidenced by the variation in quality and detail of the drill site geologists' lithology logs.

2) The laboratory descriptions of the core at a one foot interval was very useful for the LLNL analysis. Missing though was a "final" lithologic log created by the primary geologist for each hole. This final log pulls together all of the lithologic data into one format that can be easily reviewed. Thus, instead of a lengthy series descriptions for each foot of core, there is a very detailed lithologic log of the major lithostratigraphic units encountered in the hole. This is a standard method of organizing lithologic data, and should be practiced by personnel involved in similar remediation experiments.

3) There have been many wells drilled in the ISASDS. At this time, LLNL has not seen lithologic and/or geophysical logs from all of the boreholes, nor do we know whether or not any logs were prepared. All holes constructed on the ISASDS should have been logged, both lithologically and geophysically. Even when core is not collected, cuttings or grab samples should be taken. If samples simply are impossible to collect, then at least we could attempt to interpret the lithology from even a limited suite of geophysical logs. For example, if natural gamma logs were run in each of the existing wells at the ISASDS, we could construct a reasonably detailed picture of the lithostratigraphic framework simply from the gamma logs. Theoretically, we could still run natural gamma and induction logs in the existing cased and grouted holes. With the appropriate data from the large number of wells in the ISASDS, we could construct a very detailed, defensible picture of the subsurface and provide a more accurate basis for modeling contaminant - extraction. 


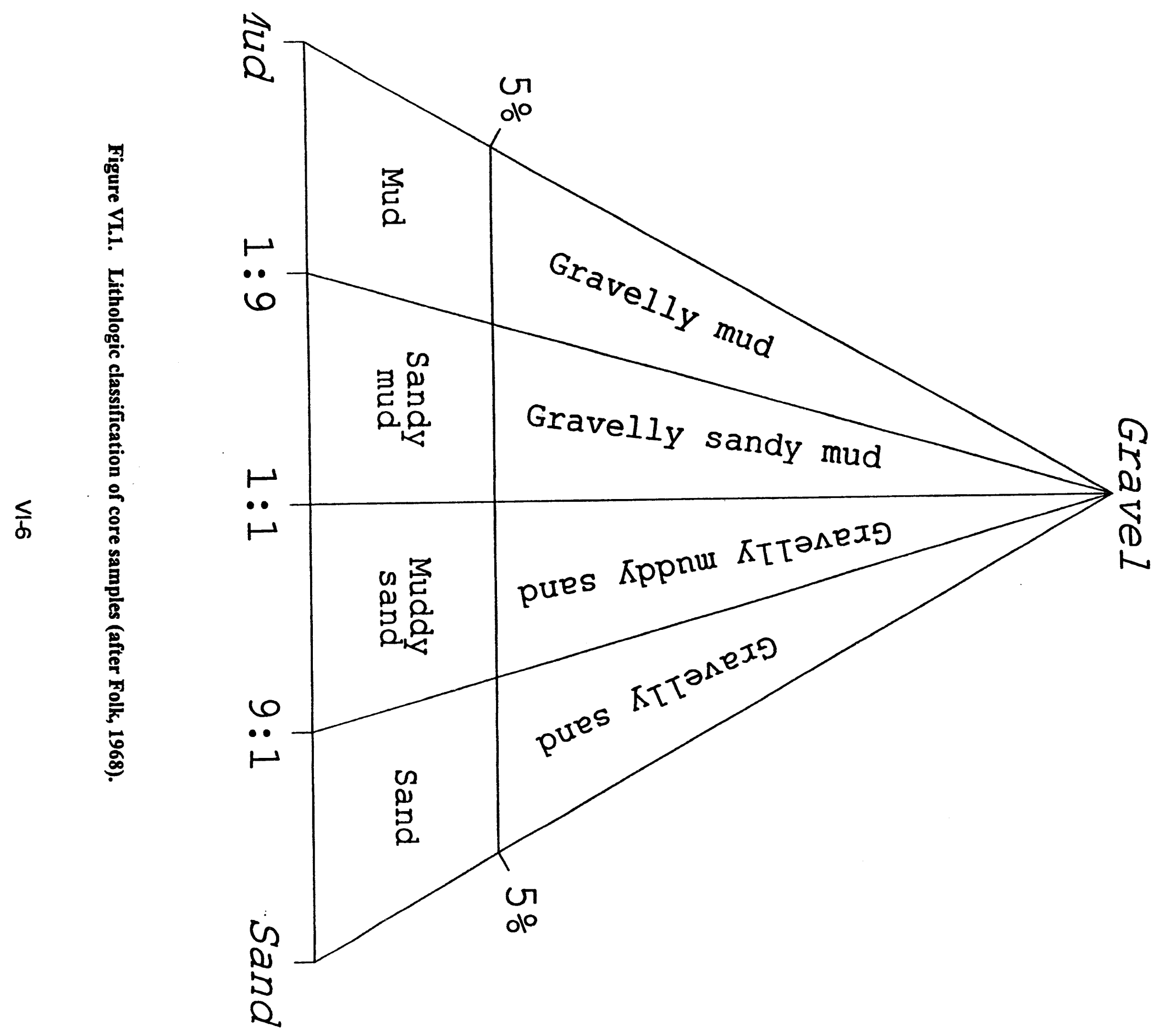




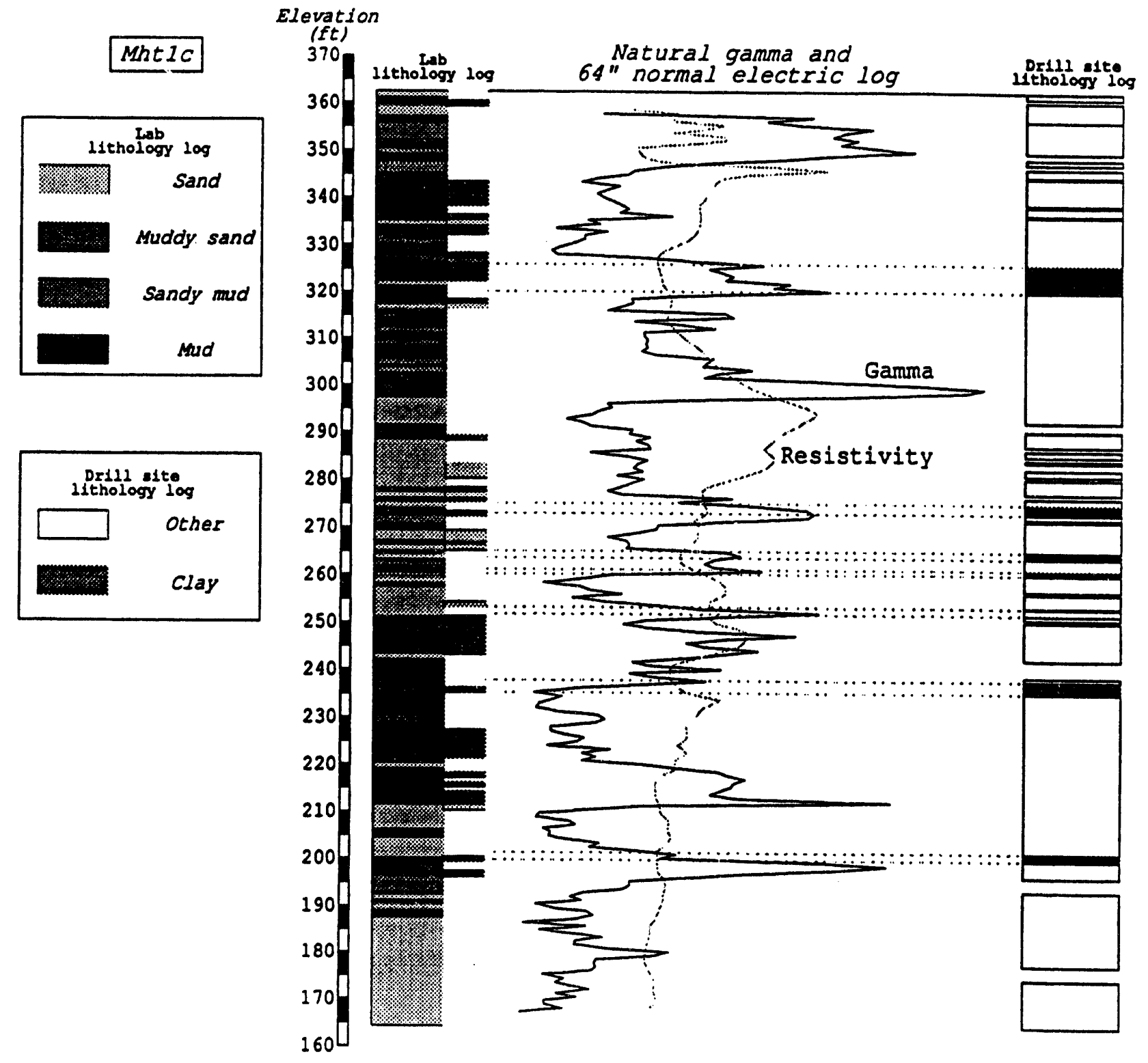

Figure VI.2. Lithologic section for MHT1c. The lithology displayed on the left column was generated in the laboratory using a binocular microscope, while the lithology on the right was described by the drill site geologist at the rig, during drilling. Other the clay beds are delineated on this section. The boxes extending from the right edge of the lab lithologic log denote beds containing $>5 \%$ gravel. The natural gamma and $64 "$ normal electric log are plotted for correlation purposes. The scales are not included on this plot. 


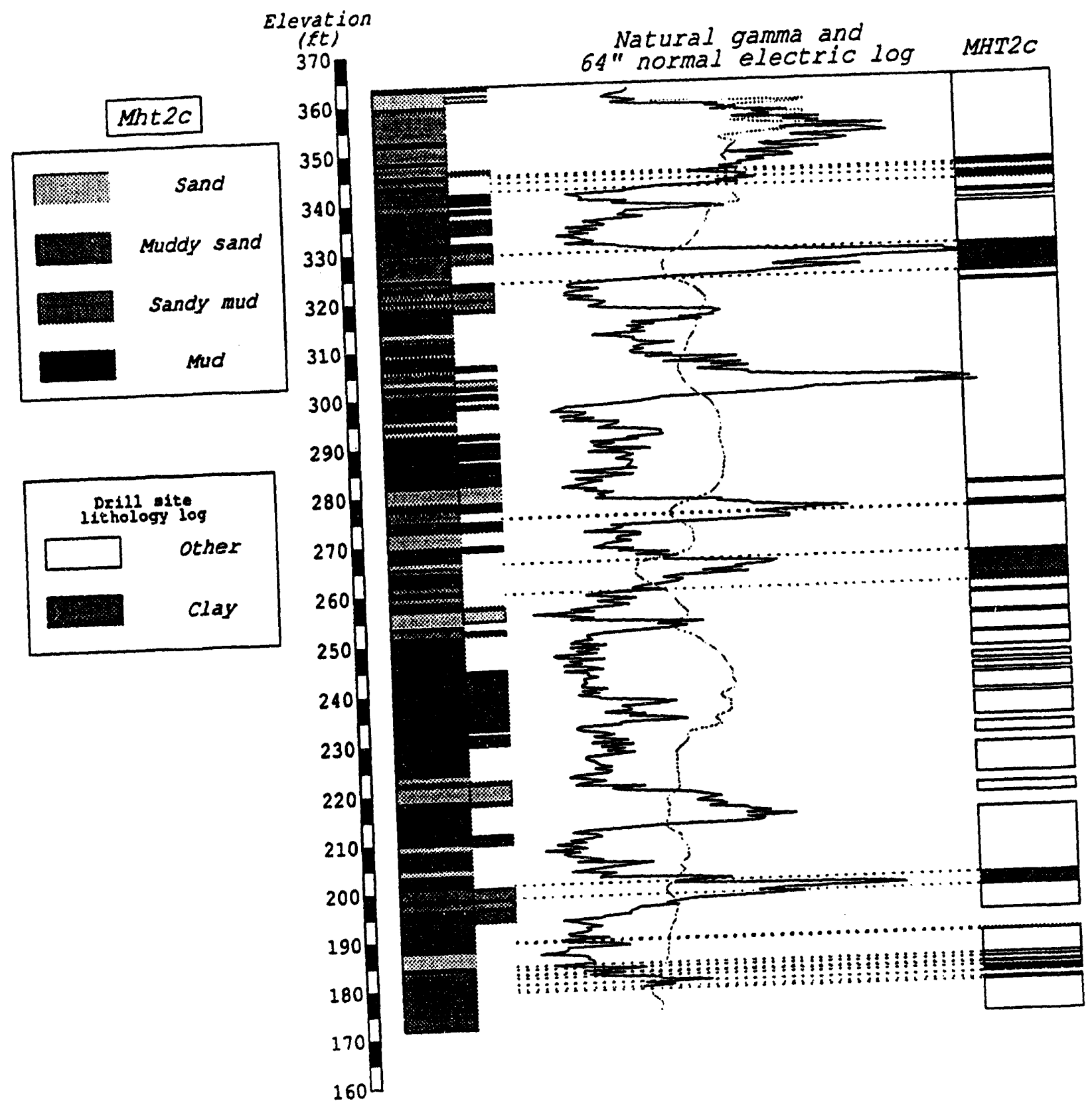

Figure VL.3. Lithologic section for MHT2c. The lithology displayed on the left column was generated in the laboratory using a by the drill site geologist while the lithology on the right was describeds are delineated on this at the rig, during drilling. Other the claygh edge of the lab lithologic section. The boxes extending from the The natural gamma and $64^{\prime \prime}$ log denote beds containing $>5 \%$ gravel. The normal electric $\log$ are plott 


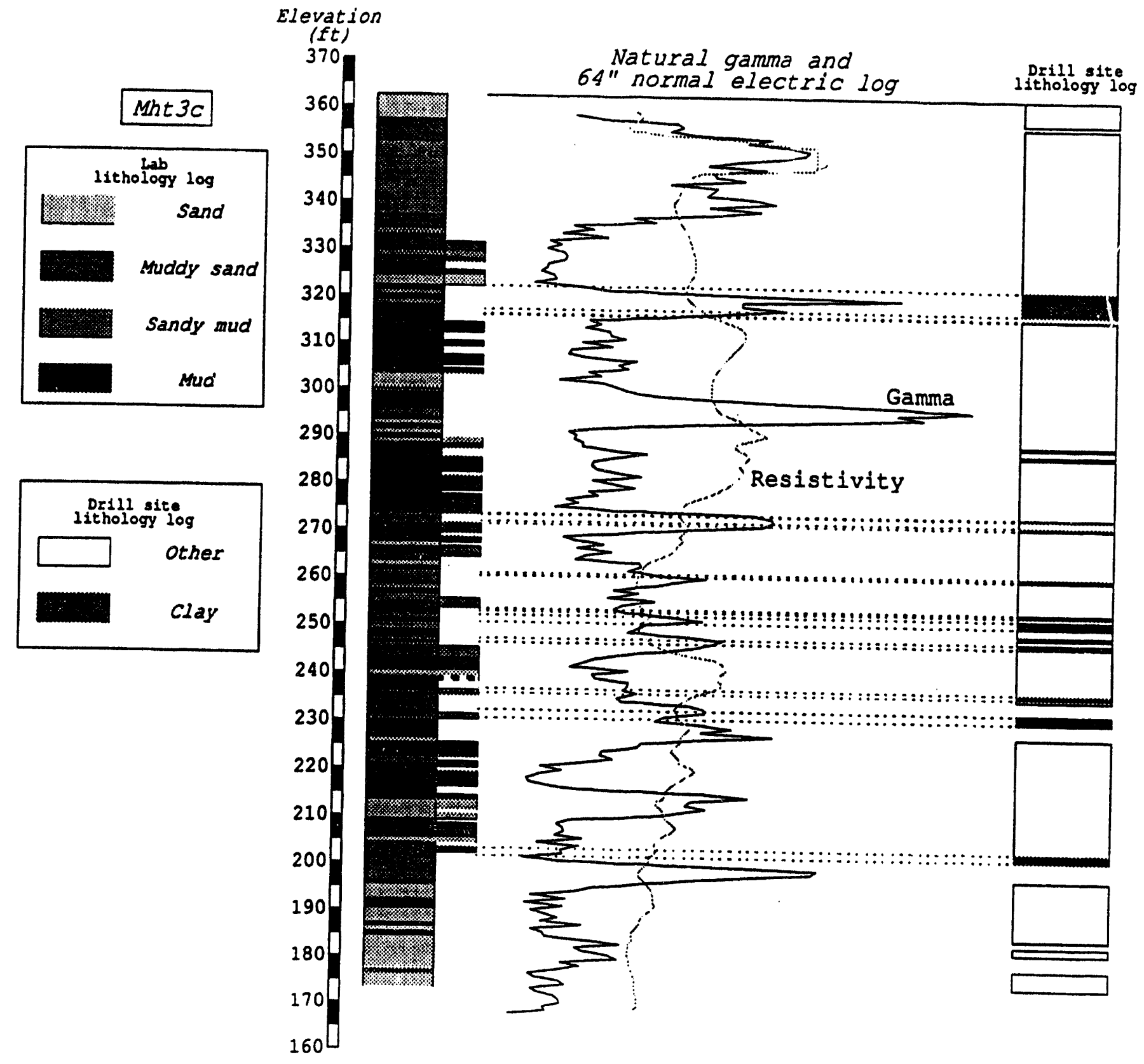

Figure VI.4 Lithologic section for MHT3c. The lithology displayed on the left column was generated in the laboratory using a binocular microscope, while the lithology on the right was described by the drill site geologist at the rig, during drilling. Other the clay beds are delineated on this section. The boxes extending from the right edge of the lab lithologic log denote beds containing $>5 \%$ gravel. The natural gamma and $64 "$ normal electric log are plotted for correlation purposes. The scales are not included on this plot. 


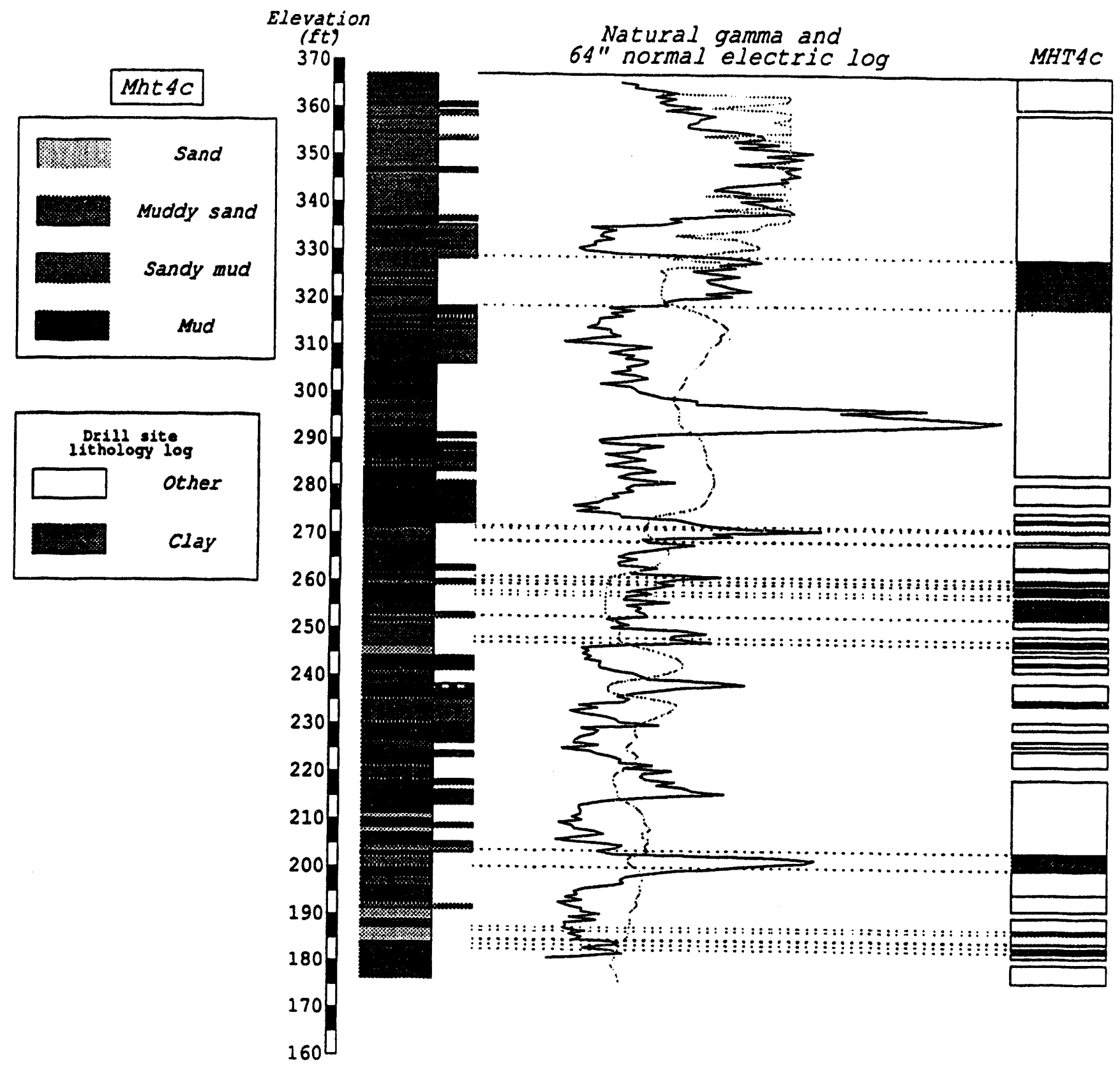

Figure VL.5. Lithologic section for MHT4c. The lithology displayed on the left column was generated in the laboratory using a binocular microscope, while the lithology on the right was described by the drill site geologist at the rig, during drilling. Other the clay beds are delineated on this section. The boxes extending from the right edge of the lab lithologic log denote beds containing $>5 \%$ gravel. The natural gamma and $64 "$ normal electric log are plotted for correlation purposes. The scales are not included on this plot. 


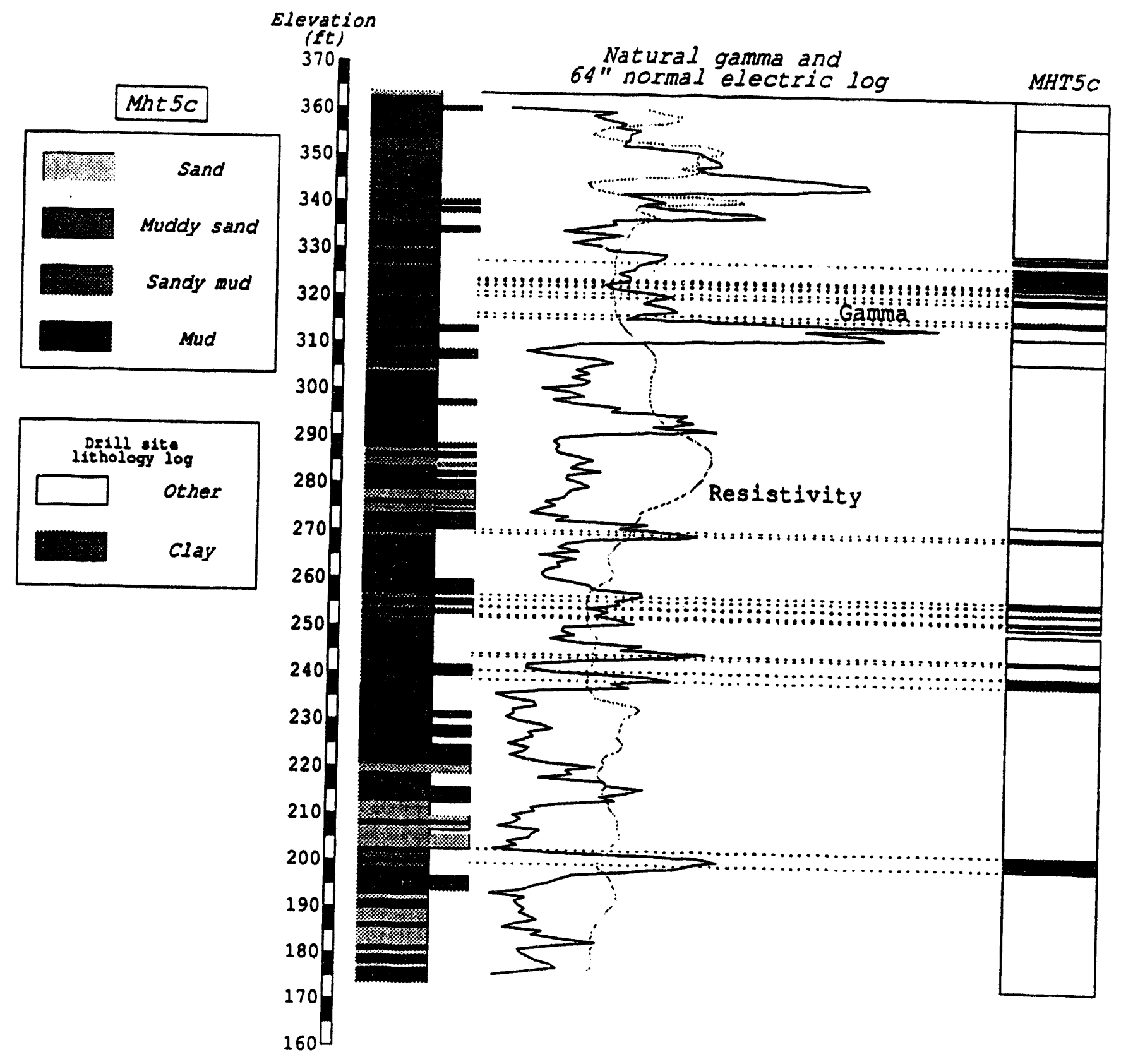

Figure VI.6. Lithologic section for MHTSc. The lithology displayed on the left column was generated in the laboratory using a binocular microscope, while the lithology on the right was described by the drill site geologist at the rig, during drilling. Other the clay beds are delineated on this section. The bores extending from the right edge of the lab lithologic log denote beds containing $>5 \%$ gravel. The natural gamma and $64^{\prime \prime}$ normal electric log are plotted for correlation purposes. The scales are not included on this plot. 


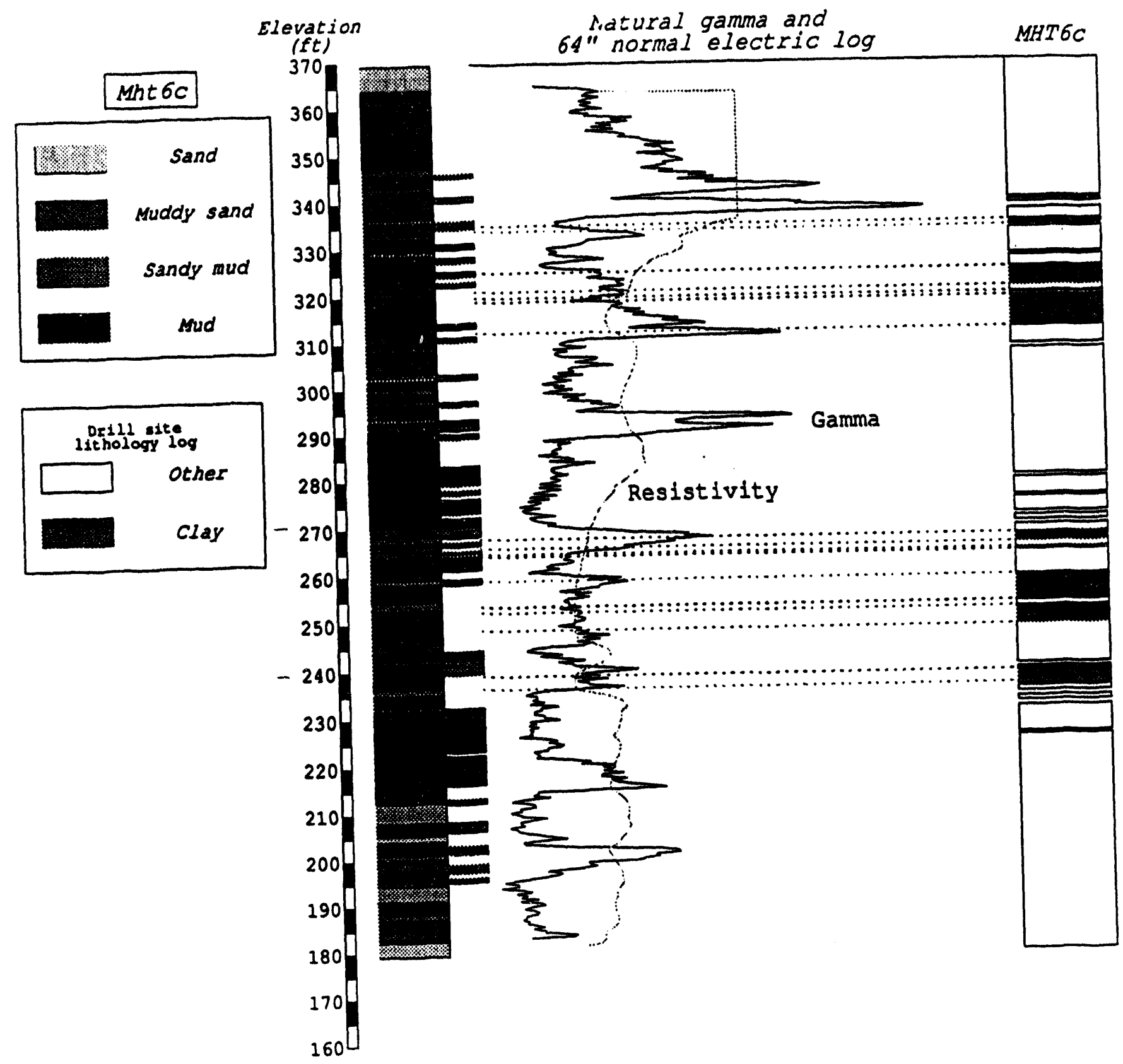

Figure VI.7. Lithologic section for MHT6c. The lithology displayed on the left column was generated in the laboratory using a binocular microscope, while the lithology on the right was described by the drill site geologist at the rig, during drilling. Other the clay beds are delineated on this section. The boxes extending from the right edge of the lab lithologic log denote beds containing $>5 \%$ gravel. The natural gamma and $64 "$ normal electric log are plotted for correlation purposes. The scales are not included on this plot. 


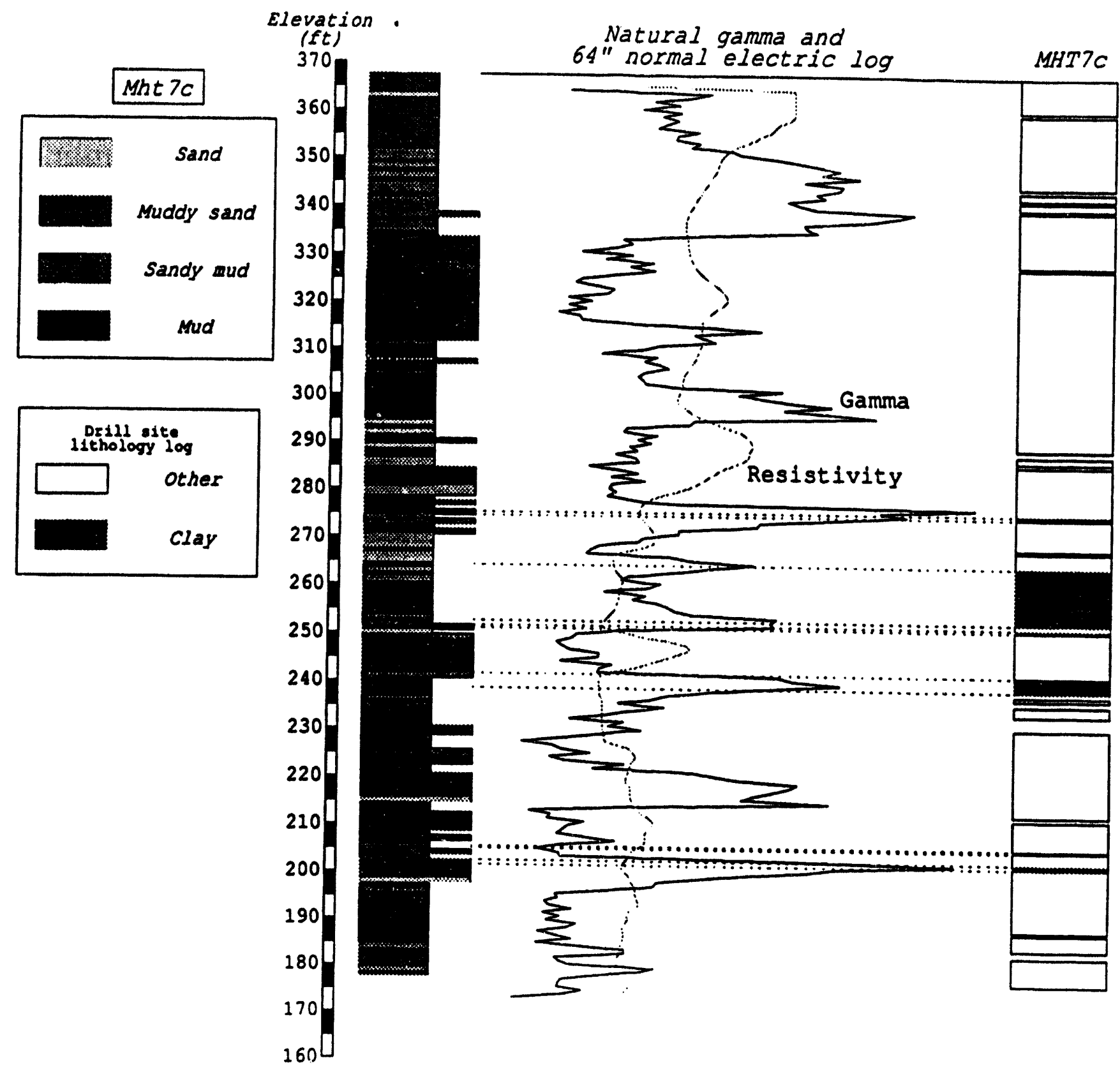

Figure VL.8. Lithologic section for MHT7c. The lithology displayed on the left column was generated in the laboratory using a binocular microscope, while the lithology on the right was described by the drill site geologist at the rig, during drilling. Other the clay beds are delineated on this section. The boxes extending from the right edge of the lab lithologic log denote beds containing >5\% gravel. The natural gamma and 64" normal electric log are plotted for correlation purposes. The scales are not included on this plot. 


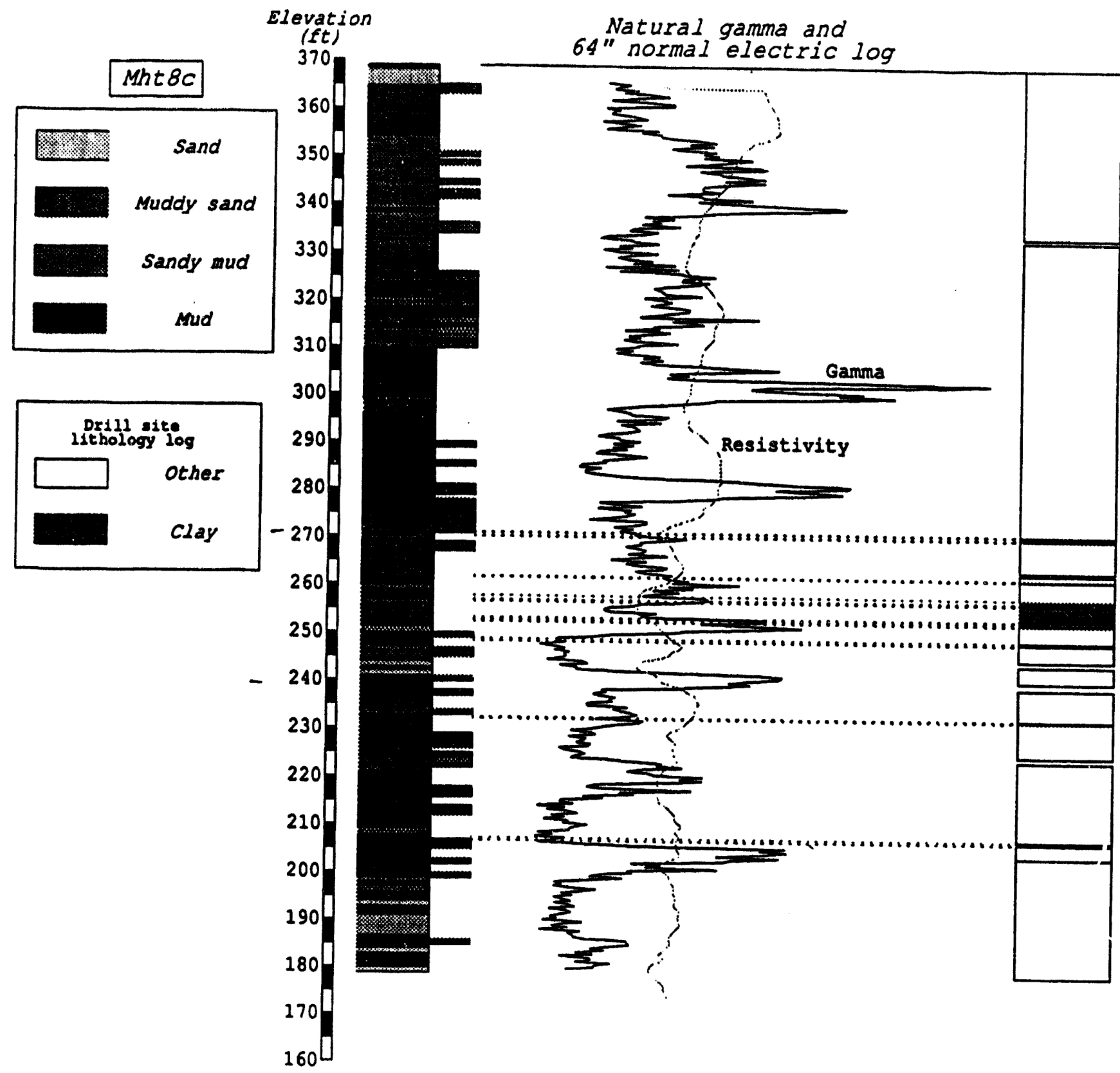

Figure VI.9. Lithologic section for MHT8c. The lithology displayed on the left column was generated in the laboratory using a binocular microscope, while the lithology on the right was described by the drill site geologist at the rig, during drilling. Other the clay beds are delineated on this section. The boxes extending from the right edge of the lab lithologic log denote beds containing $>5 \%$ gravel. The natural gamma and $64^{\prime \prime}$ normal electric log are plotted for correlation purposes. The scales are not included on this plot. 


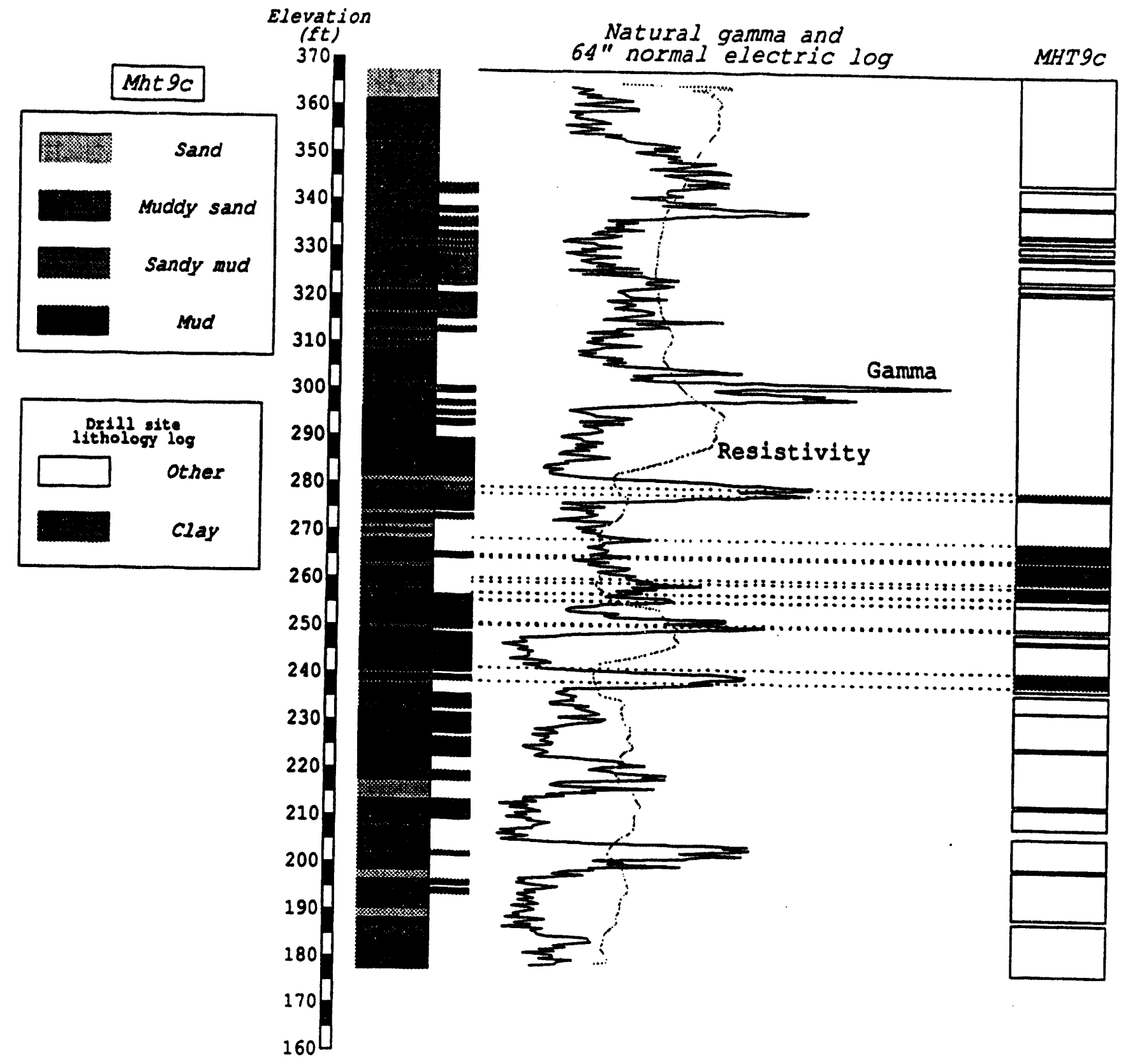

Figure VL.10. Lithologic section for MHT9c. The lithology displayed on the left column was generated in the laboratory using a binocular microscope, while the lithology on the right was described by the drill site geologist at the rig, during drilling. Other the clay beds are delineated on this section. The boxes extending from the right edge of the lab lithologic log denote beds containing >5\% gravel. The natural gamma and $64 "$ normal electric log are plotted for correlation purposes. The scales are not included on this plot. 


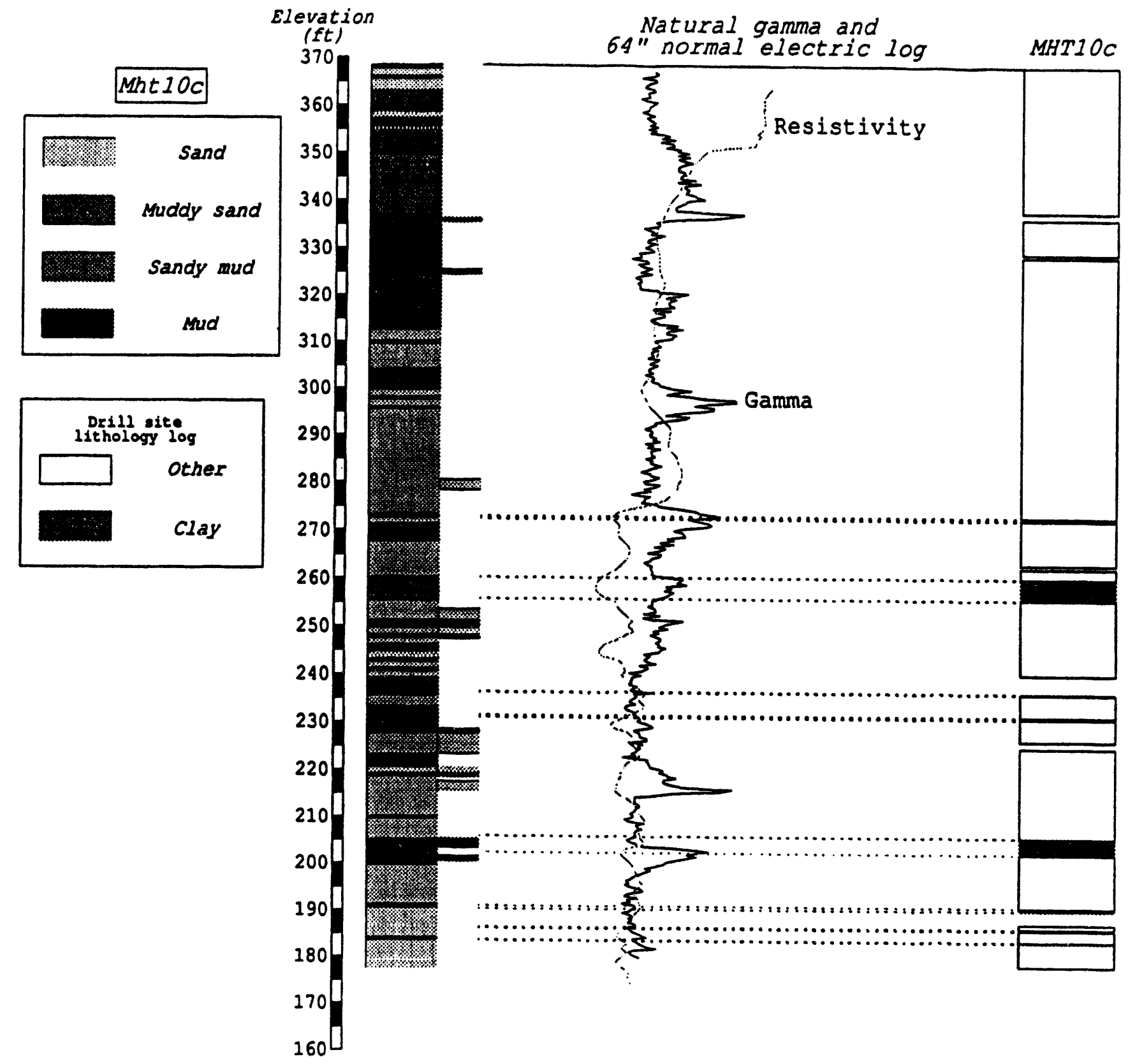

Figure VL.11. Lithologic section for MHT10c. The lithology displayed on the left column was generated in the laboratory using a binocular microscope, while the lithology on the right was described by the drill site geologist at the rig, during drilling. Other the clay beds are delineated on this section. The boxes extending from the right edge of the lab lithologic log denote beds containing $>5 \%$ gravel. The natural gamma and $64^{\prime \prime}$ normal electric log are plotted for correlation purposes. The scales are not included on this plot. 


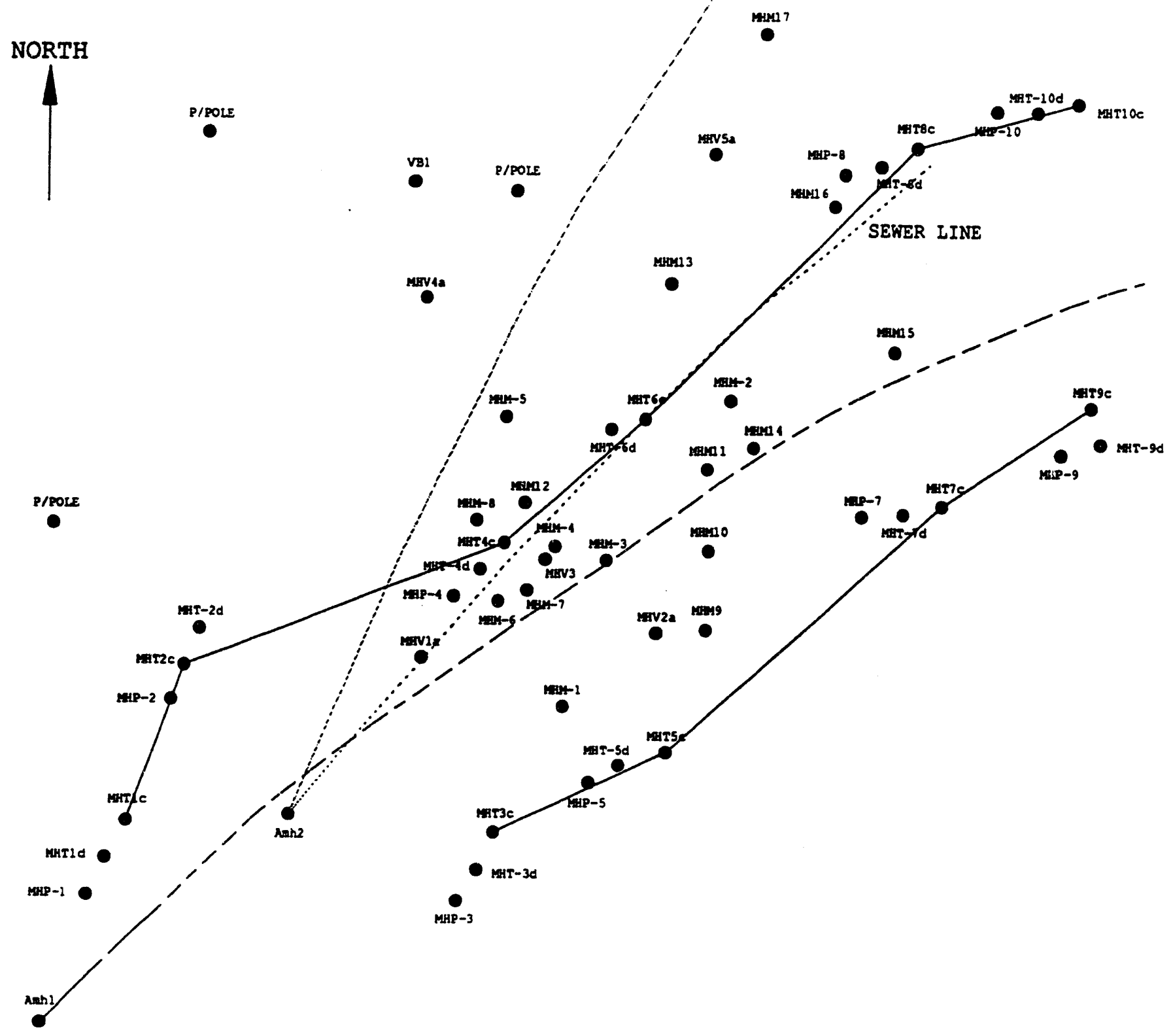

Figure VL.12. Plan map of the In situ air stripping demonstration site. Most of the existing wells are plotted, although we do not have the coordinates for some wells. The lines emanating from Amh1 and Amh 2 define the lateral extent of these horizontal wells. Also note the location of the lines of section for geologic cross sections $A-A^{\prime}$ and $B-B^{\prime}$. 


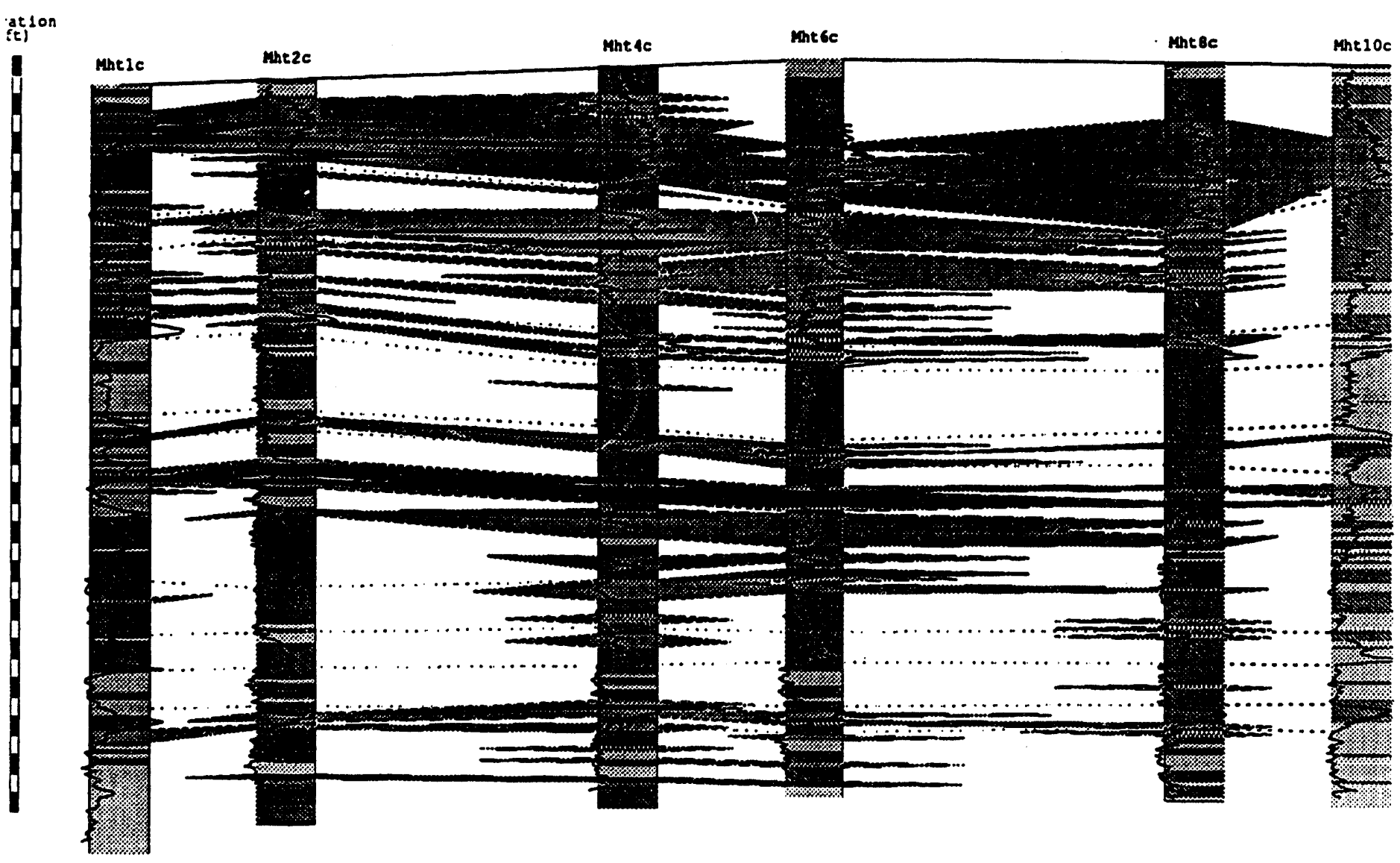

Figure VL.13. Geologic cross section A-A'. This lithostratigraphic cross section was constructed using the lithologic data that was generated in the lab with a binocular microscope. The correlations are based on depth and lithology, while honoring the character of the gamma logs, which are plotted on the stratigraphic columns. 


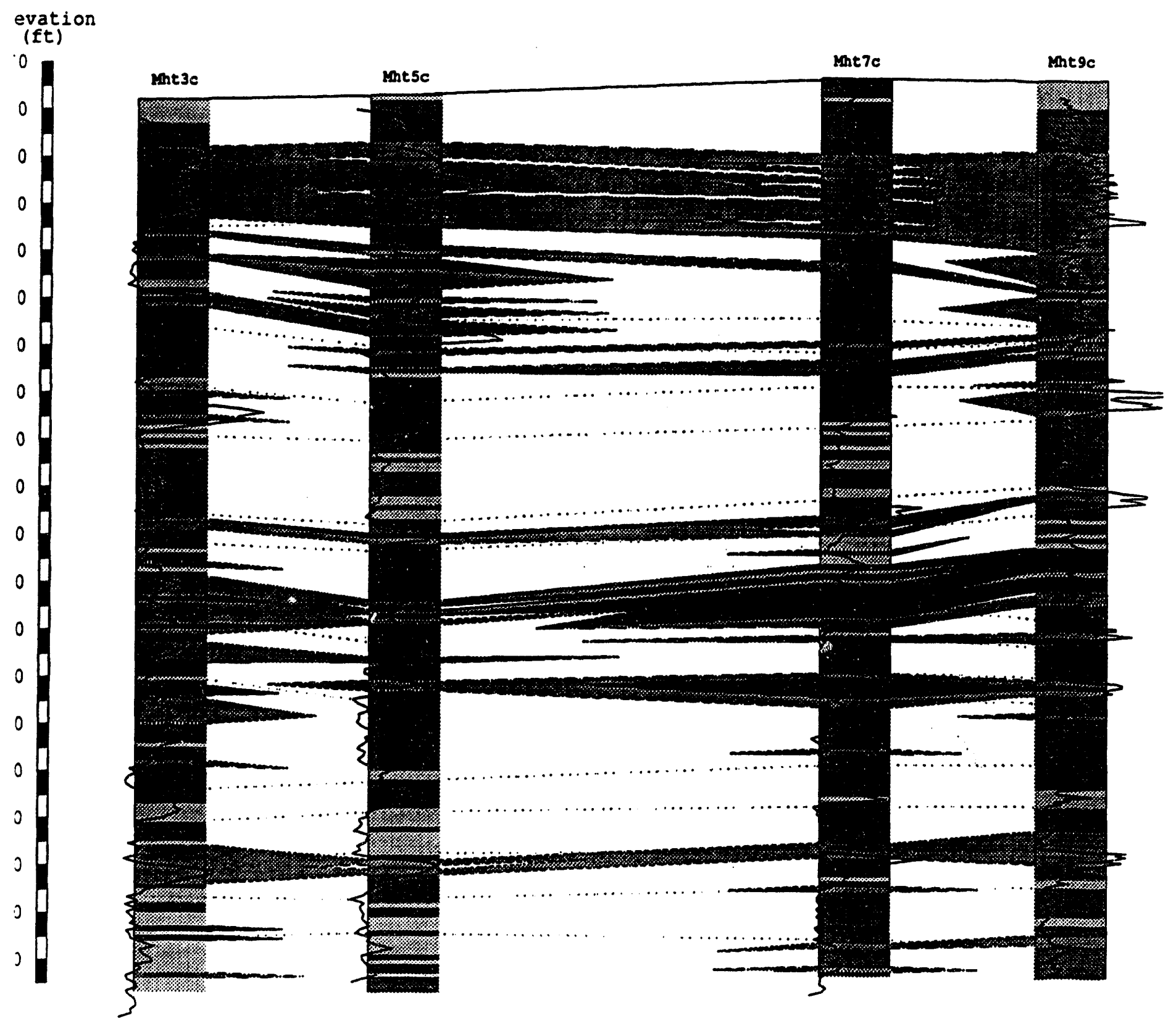

Figure VL.14. Geologic cross section B-B'. This lithostratigraphic cross section was constructed using the lithologic data that was generated in the lab with a binocular microscope. The correlations are based on depth and lithology, while honoring the character of the gamma logs, which are plotted on the stratigraphic columns. 


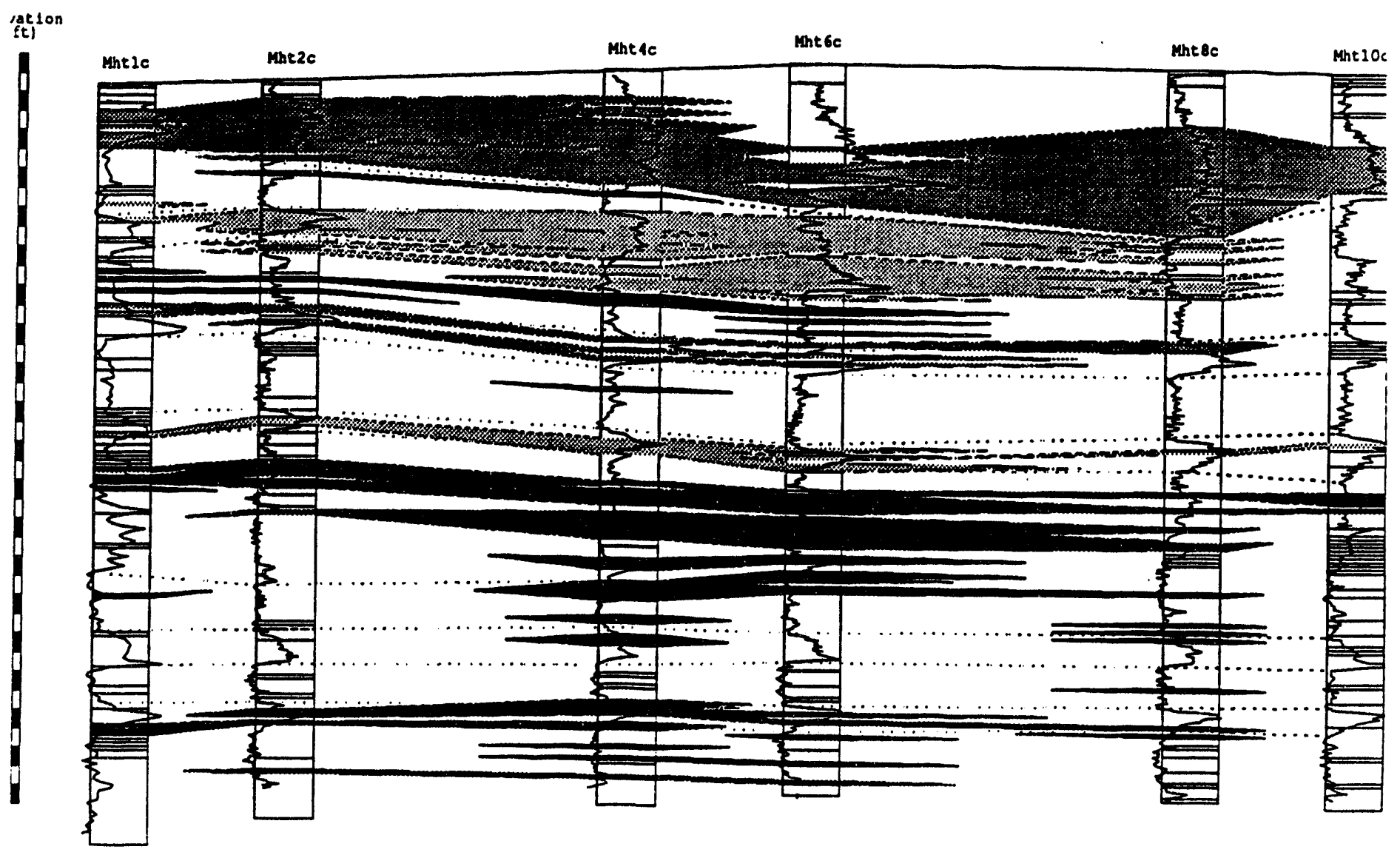

Figure VL.15. Geologic cross section A-A'. On this and the following cross section, we have identified unique mud horizons that can be correlated between the two lines of section. The mud units are color coded for ease of correlation between the sections. The surfaces of these units are then modeled with an interactive surface modeling code, in an attempt to construct the three-dimensional stratigraphic framework. 


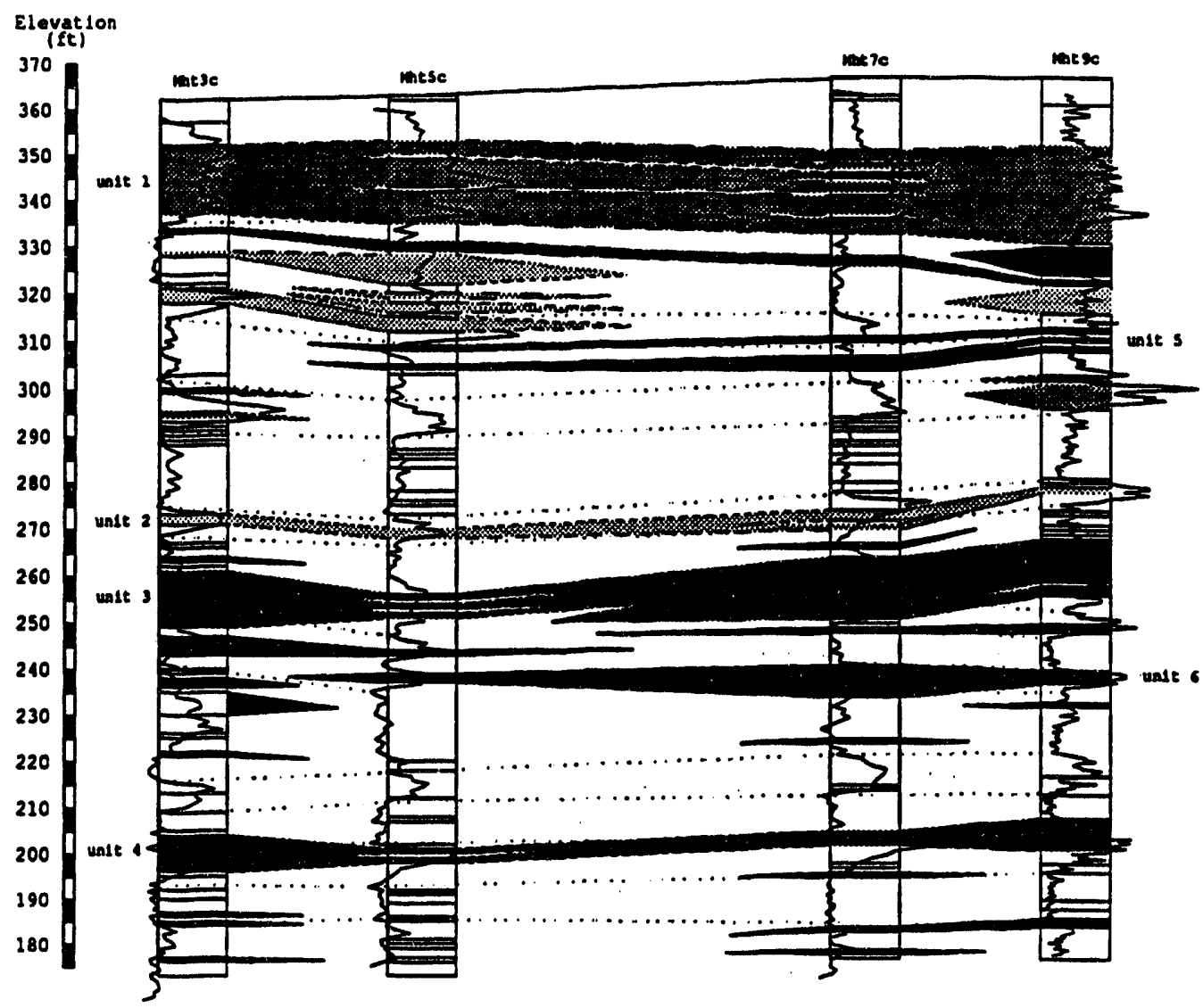

Figure VL.16. Geologic cross section B-B'. On this and the previous cross section, we have identified unique mud horizons that can be correlated between the two lines of section. The mud units are color coded for ease of correlation between the sections. The surfaces of these units are then modeled with an interactive surface modeling code, in an attempt to construct the three-dimensional stratigraphic framework. 


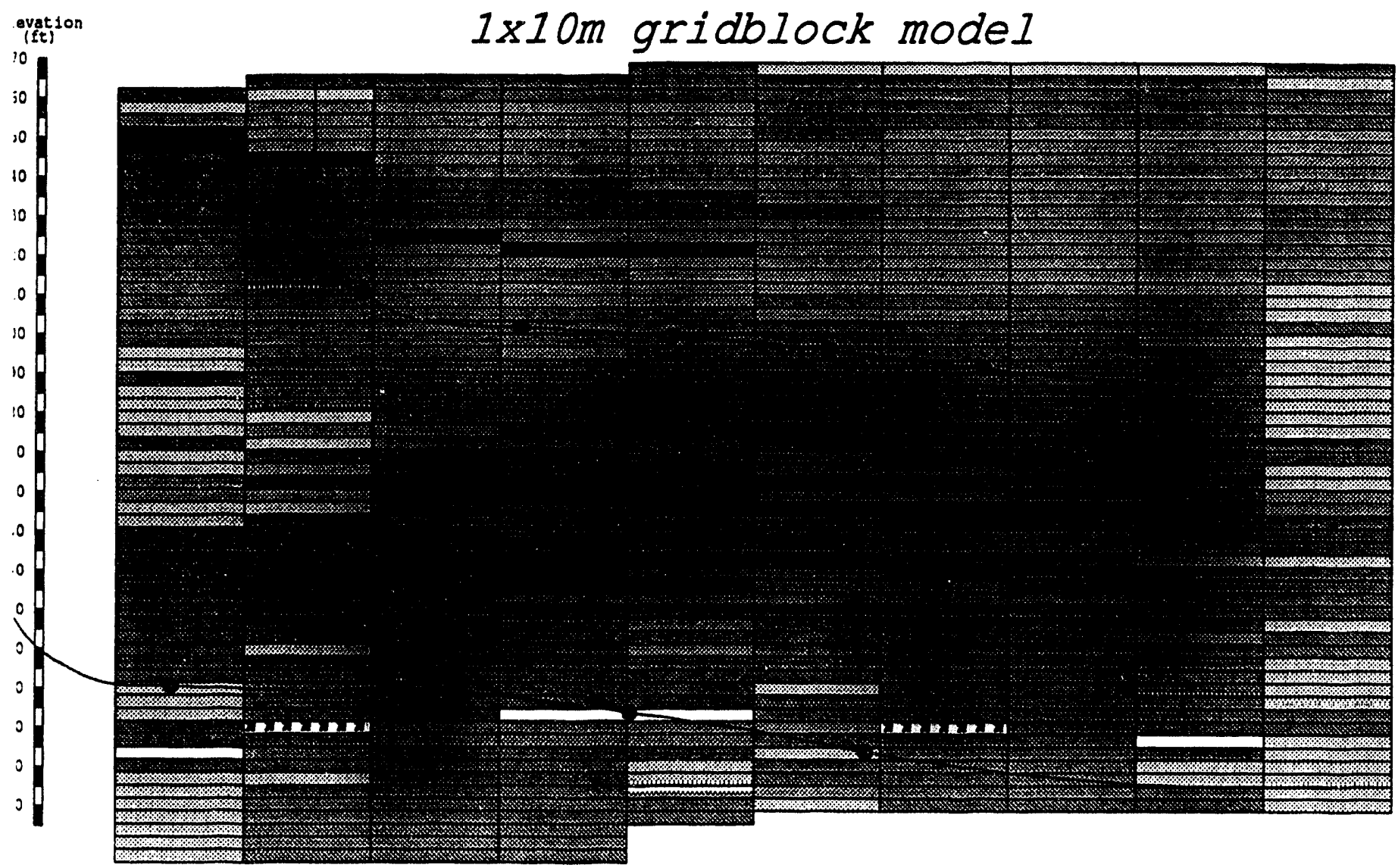

Figure VL17. Grid model of geologic cross section A-A'. This model is composed of $1 \mathrm{~m} \times 10 \mathrm{~m}$ rectangular grid blocks, in which the individual grid blocks have been assigned a specific lithology. This lithology represents the dominant lithology that is intersected in the cross section beneath the grid. permeability is also assigned to each gridlock, as discussed in the text of the report. 


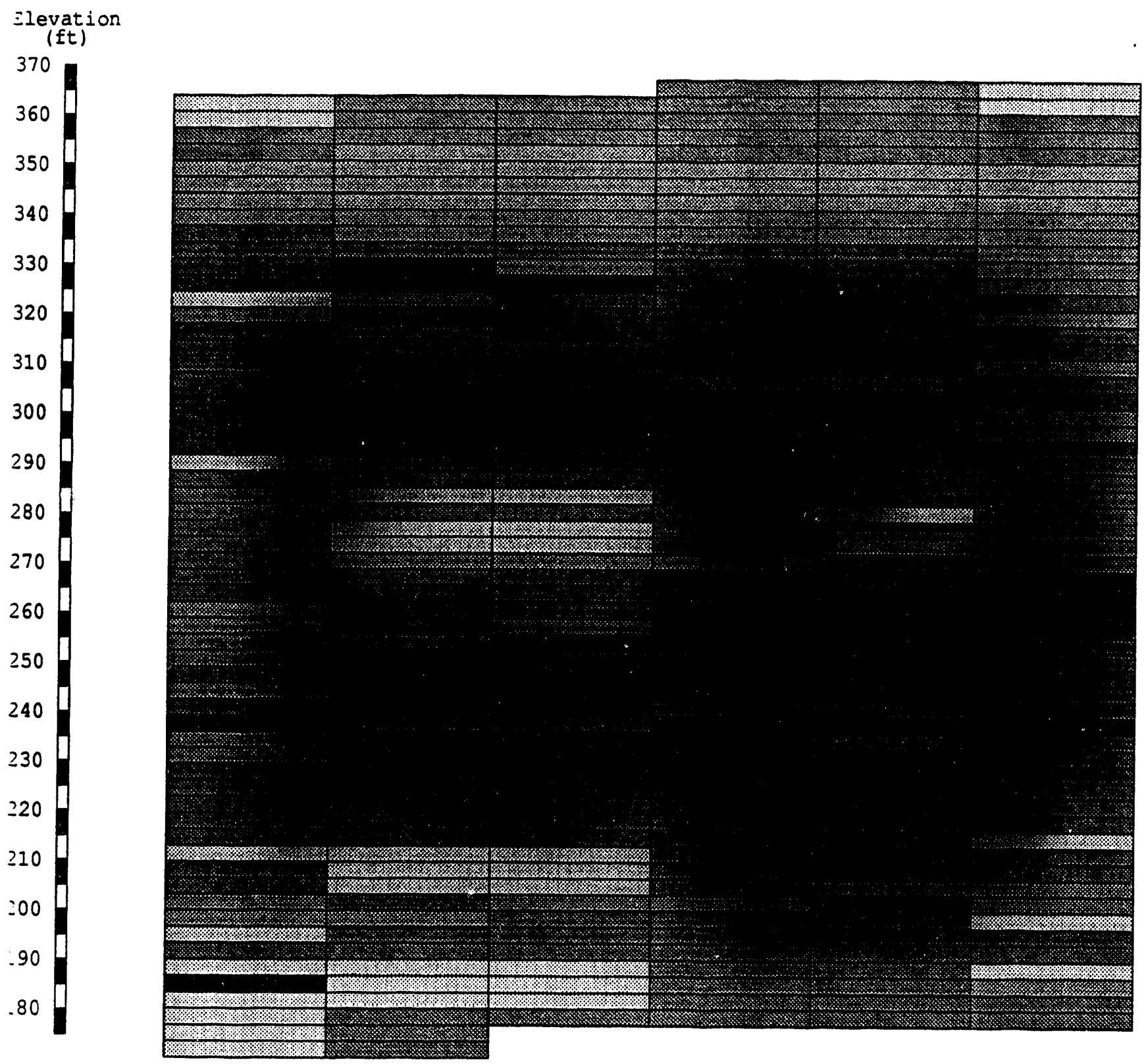

Figure VI.18. Grid model of geologic cross section B-B'. This model is composed of $1 \mathrm{~m} \times 10 \mathrm{~m}$ rectangular grid blocks, in which the individual grid blocks have been assigned a specific lithology. This lithology represents the dominant lithology that is intersected in the cross section beneath the grid. permeability is also assigned to each gridlock, as discussed in the text of the report. 


\section{GeOPHYSICAL ANALYSES}

In the previous part of this section, the geophysical logs furnished to us provided part of the information upon which we based our stratigraphic correlation for the ISASDS. They were not used quantitatively except to provide information about bed thicknesses. In the following, we describe briefly the use of a suite of geophysical logs to determine porosity through the section and water saturation in the vadose zone, two of the more important sediment parameters required for flow and transport modeling.

\section{Hole Information}

The hole was augured down to the water table using no drilling fluids. Below the fluid level, a standard mud-rotary drilling system was used to drill down to TD (total depth). Prior to logging, the hole was filled to the surface with water. No casing was set.

\section{Logging Program:}

The geophysical well logs were obtained by Graves Environmental and Geotechnical Services 1/24/90. Three services were run to acquire neutron porosity, density, and resistivity logs. These logs will now be discussed.

\section{DENSTYYLOGS}

The data were acquired using the Century Geophysical Corp. 9030A logging tool, a 2.2" diameter mandrel incorporating the following sensors: a gamma ray detector to measure the natural radioactivity of the formation; a $5^{n}$ focused guard resistivity electrode; caliper arm to measure the hole diameter, and a scintillation detector to measure the gamma rays originating from the $125 \mathrm{mCi}$ Cesium 137 source housed at the bottom of the tool.

The caliper $\log$ provides the hole diameter versus depth. The hole is roughly $7^{n}$ in diameter, highly nigose from surface to $\sim 118 \mathrm{ft}$. Below $118 \mathrm{ft}$, the hole size decreases to a diameter of $-4.25^{\prime \prime}$ and the rugosity disappears. A large washout to $9^{n}$ is indicated by the caliper $\log$ at a depth of $87 \mathrm{ft}$.

The density is determined using a single scintillation detector which measures the total energy of the gamma rays scattered from the source. The depth of investigation is from 3-8". One disadvantage of utilizing a single-detector tool is that the measurement is greatly affected by the borehole environment. More sophisticated density tools use two detectors, one positioned close to the source and another placed further away. A two-detector system allows the measurement to be corrected for borehole effects by using both count rates. The density measurement from a single-detector tool cannot be corrected for borehole effects. Thus, when the hole is large or rugose, the measurements are adversely affected; they are usually lower the true bulk density. For example, the average bulk density is $\sim 1.7 \mathrm{~g} / \mathrm{cc}$ which appears to be a low bulk density. It is quite probable that the tool may not be in sufficient contact with the borehole wall, resulting in a gap between the detector and the formation and lower density measurements. Century Geophysical Corp. does not have a method to correct the density log for borehole effects.

A density-derived porosity is based on the density values for an assumed sand matrix and water as the borehole fluid:

$$
\begin{gathered}
\rho_{m a}=2.65 \mathrm{~g} / \mathrm{cm}^{3} \\
\rho_{f}=1.0 \mathrm{~g} / \mathrm{cm}^{3}
\end{gathered}
$$

The equation for computing the density-derived porosity is

$$
\phi_{d}=\frac{\rho_{b}-\rho_{m a}}{\rho_{f}-\rho_{m a}}
$$


In Eq VI.4, $\rho_{b}$ is the bulk density obtained directly from the density $\log$ measurements, and $\phi_{d}$ is the porosity derived from them. As stated above, the density measurements are adversely affected by hole size and rugosity. When the detector face is pulled away from the borehole wall due to washouts and other factors, the density will be artificially low. When the density-derived porosity is used to determine the total porosity and water saturation, these calculations also reflect the artificially low density readings.

\section{NEUTRON LOGS}

The neutron porosity was determined using the Century Geophysical Corp. 9055A logging tool, a 1.8" diameter mandrel featuring a scintillation detector for measuring the natural radioactivity of the formation and one Helium 3 detector for sensing neutrons scattered from a $1 \mathrm{Ci}$ AmBe241 neutron source. The neutron counts are recorded in API units (normalized to the tool calibration). The initial neutron porosity values looked suspiciously low and erratic. For example, the data jumped by as much as 20 percent in a single measurement. The data are spiky, and in several zones the measurements were obviously erroneous (abnormally high neutron count rates resulting in negative porosity readings). It looked as though the tool may have been malfunctioning in these zones.

A borehole correction chart, dated 1978, was furnished by Century Geophysical Corp. to correct the porosity measurements for hole size effects. The chart data were renormalized depending on the tool calibration, re-plotted, and curves were fitted to represent the relationship between porosity and normalized count rates for varying hole sizes. On average, the hole size correction increased the porosity readings by 37 percent of the value before correction. 


\section{Porosity and Invaded Zone Water Saturation Calculations}

The total porosity and water saturation in the invaded zone (near the well-bore) are determined from both the density and neutron porosity values. When the formation is completely water-saturated, the porosity derived from the density log should be equal to the porosity derived from the neutron log. In the vadose zone, however, the apparent neutron porosity will be lower, since the neutron log "sees" the amount of hydrogen in the sediment. The difference between the apparent neutron porosity and the porosity derived from the density $\log$ is essentially equal to the gas-filled porosity in the sediment.

\section{TOTAL POROSITY CALCULATION}

The bulk density from the neutron $\log$ can be written

$$
\rho_{b}=\left(1-\phi_{1}\right) \cdot \rho_{m a}+\phi_{1} \cdot S_{20} \cdot \rho_{f}
$$

If Eq. (VI.๑) is solved for bulk density also and the result equated to the right hand side of Eq. (VI.5), one obtains

$$
\left(1-\phi_{d}\right) \cdot \rho_{m a}+\phi_{d} \cdot \rho_{f}=\left(1-\phi_{t}\right) \cdot \rho_{m a}+\phi_{t} \cdot \dot{S}_{x o} \cdot \rho_{f}
$$

Equation (VI.6) can then be solved for the total porosity $\phi_{i}$ :

$$
\begin{aligned}
\phi_{t} & =\phi_{d} \cdot\left(1-\frac{\rho_{f}}{\rho m a}\right)+\frac{\phi_{n}}{\rho_{m a}} \\
& =0.623 \phi_{d}+0.377 \phi_{n}
\end{aligned}
$$

The numerical values result upon substituting unity for the fluid density and 2.65 for the matrix density.

\section{INVADED ZONE WATER SATURATION}

The invaded zone water saturation, $S_{x 0}$, can be obtained after algebraic manipulation of the above equations, from

$$
S_{x o}=\frac{\rho_{m a}}{1-\frac{\phi_{d}}{\phi n}\left(1-\rho_{m a}\right)}
$$

\section{CLAY CORRECTIONS}

Because the invaded zone water saturations were reading above 100 percent, a clay correction was applied, resulting in lower values. The correction was calculated from the difference in gamma ray response from a "clean" (i.e., clay-free) zone and the gamma ray response in a clay zone. This was then used to calculate corrected neutron porosity values and, finally, total porosity values corrected for clay content. 


\section{WATER SATURATTON}

Since the water is very fresh and has a high resistivity, the electrical conductance of the sediments at the ISASDS is dominated by exchangeable cations. Under these conditions, the Waxman-Smits equation for the resistivity of shaly sands reduces to

$$
R=\frac{\left(\phi \cdot S_{w}\right)^{-1}}{B \cdot Q_{w}}
$$

In Eq. (VI.9), $R$ is the formation resistivity, $\phi$ is its fractional porosity, $S_{w}$ is the water saturation (fraction of pore volume), $B$ is the equivalent conductance of clay counterions, and $Q_{\nu b}$ is the cation exchange capacity of the sediment per unit bulk volume. We assume that this equation holds for both the invaded and uninvaded formation, and furthermore that the 16-inch normal log gives the resistivity of the invaded zone, while the 64-inch normal log records the resistivity of the uninvaded zone. Then the water saturation in the uninvaded zone is calculated from

$$
S_{w}=\frac{R_{16}}{R_{64}} \cdot S_{20} .
$$

Figures VI.19 - VI.22 summarize the results of analyzing the well logs for MHT1c. The analysis was not carried out for the other wells due to termination of funding resulting from an overall budget reduction for the integrated demonstration project. 
Hell Nome: WHTICNEU

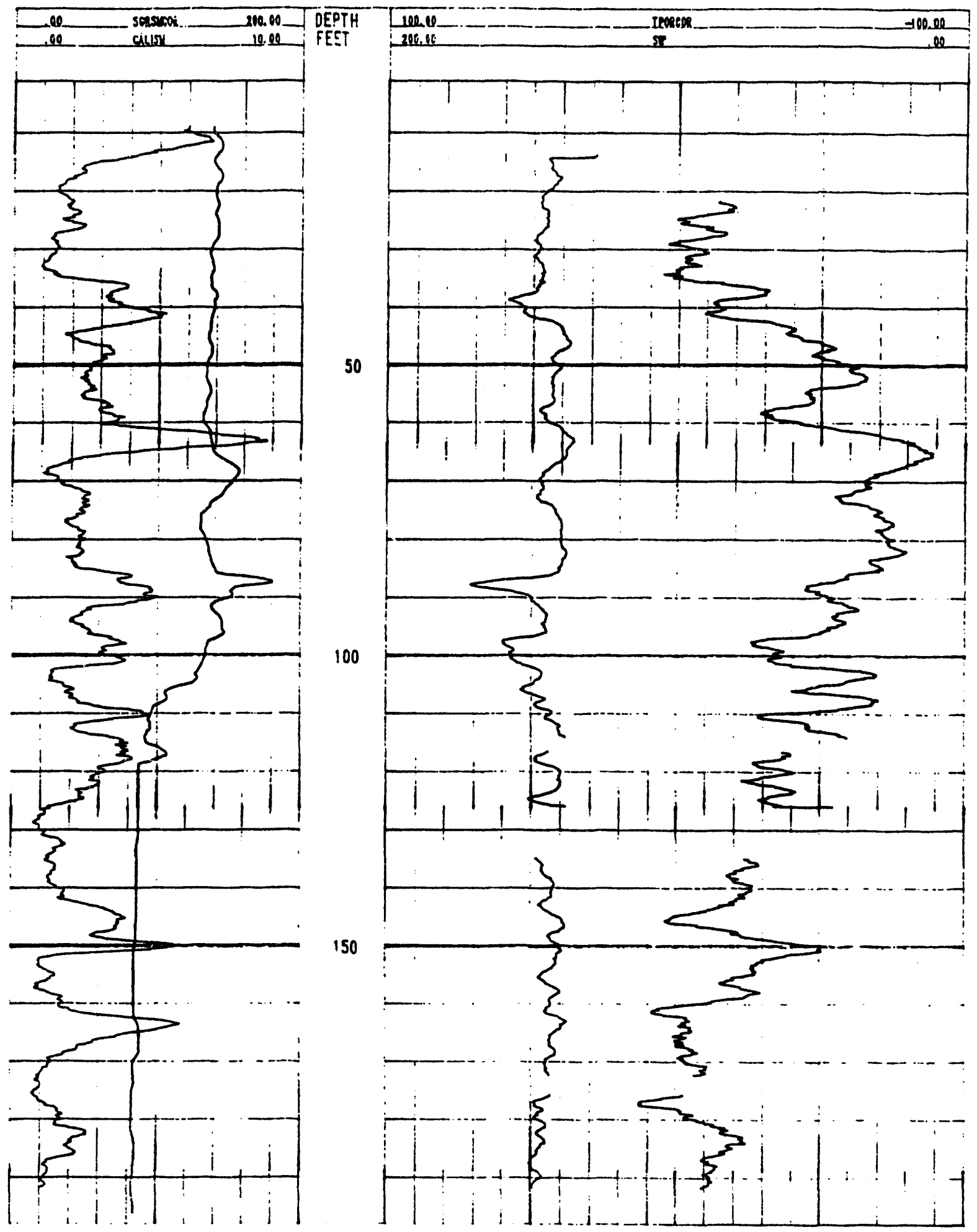

Figure VI.19. Log analysis for MHT1c. TPORCOR is the clay-corrected total porosity, $S_{w}$ ' is the shale-corrected porosity in the invaded zone, SCRSMCOR is the stripped, smoothed, borehole-corrected gamma ray response, and CALISM is the stripped, smoothed caliper log. 


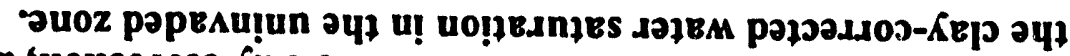

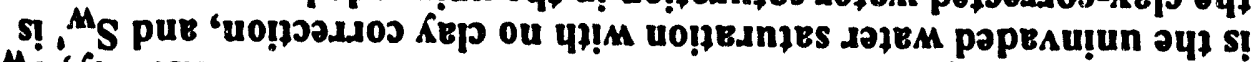

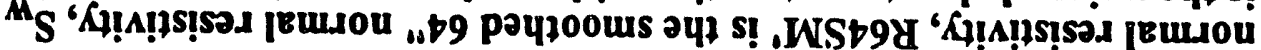

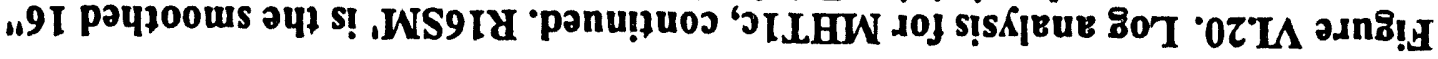

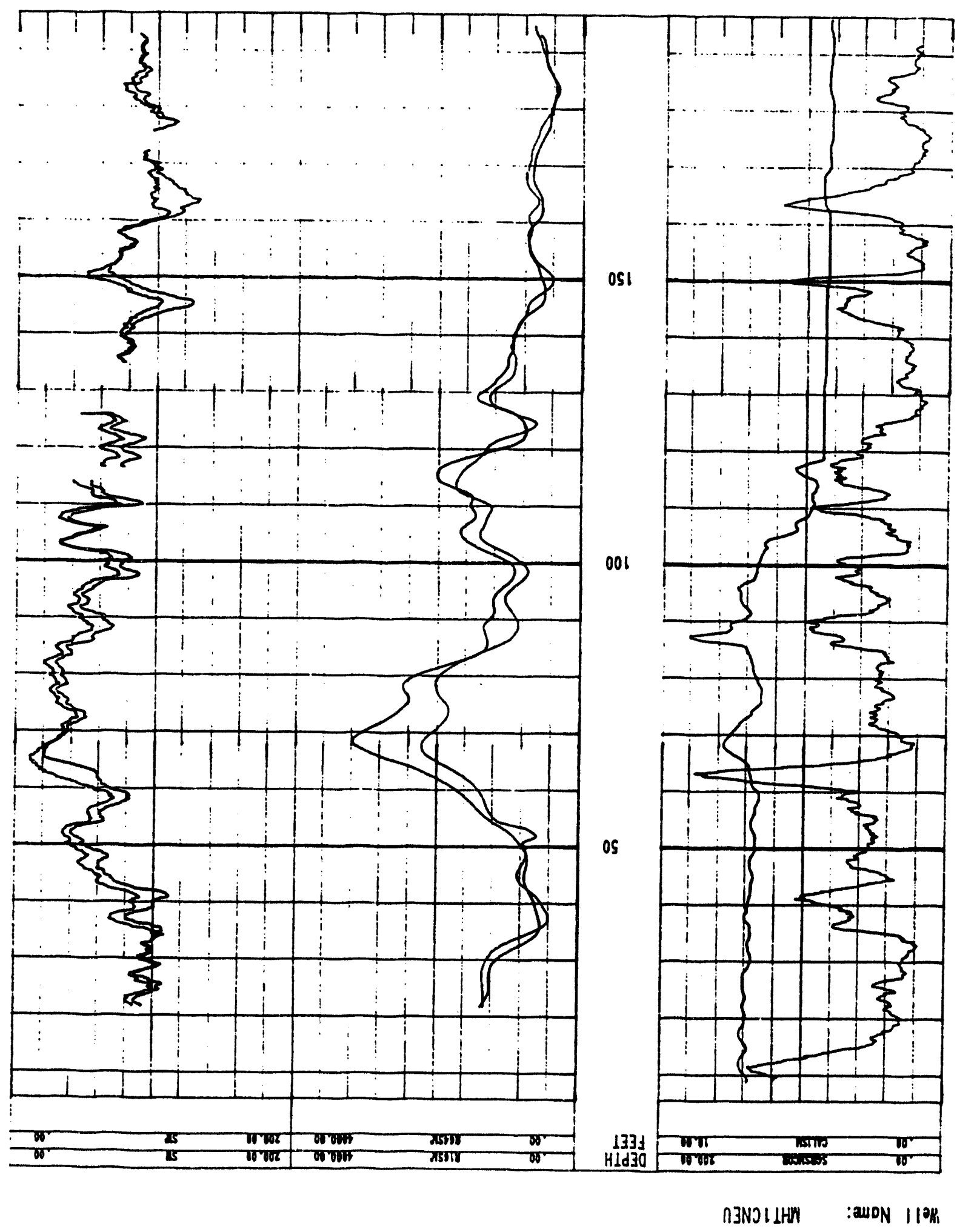


כuoz

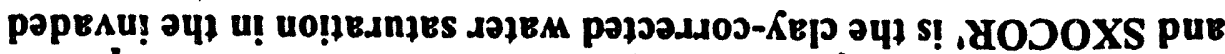

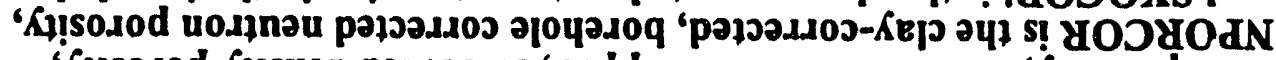

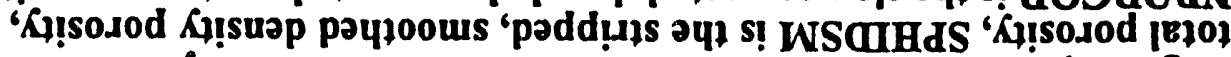

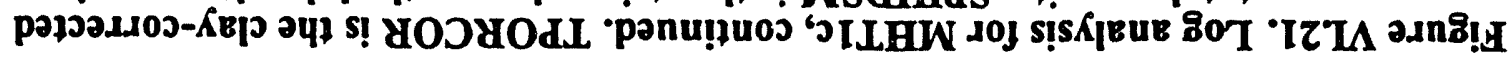

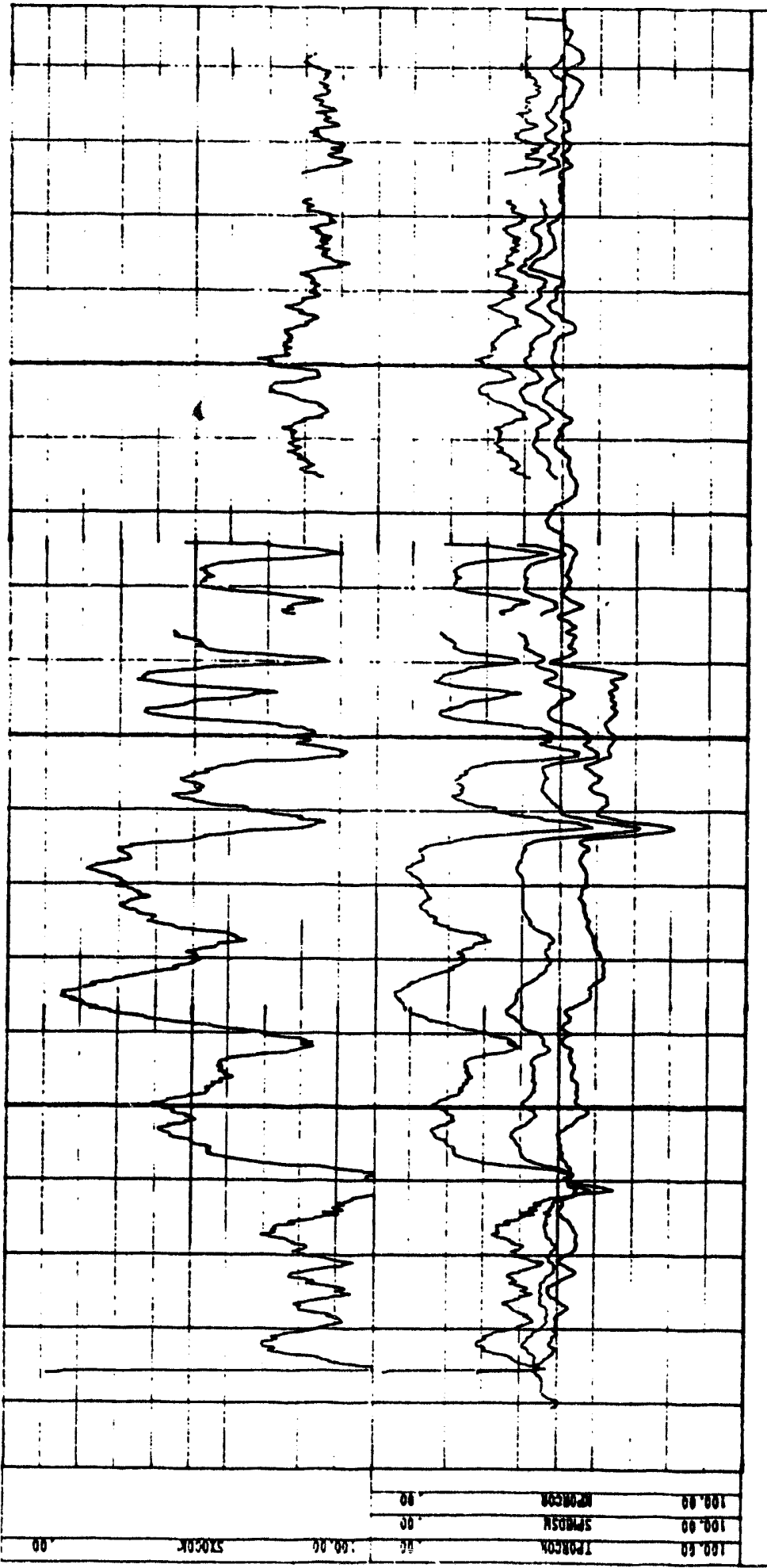

OSI

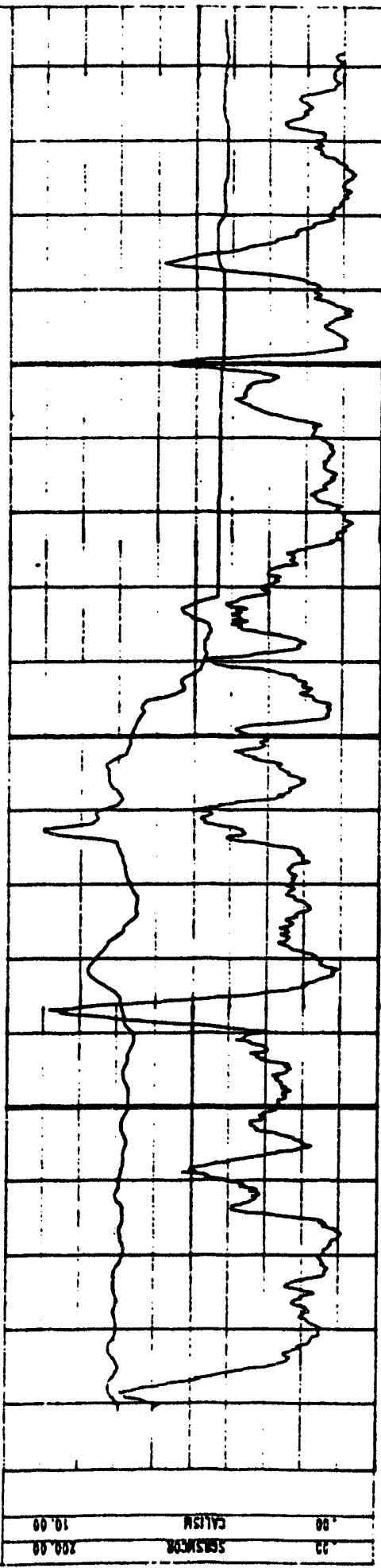


Hell Name: NHT ICNEU

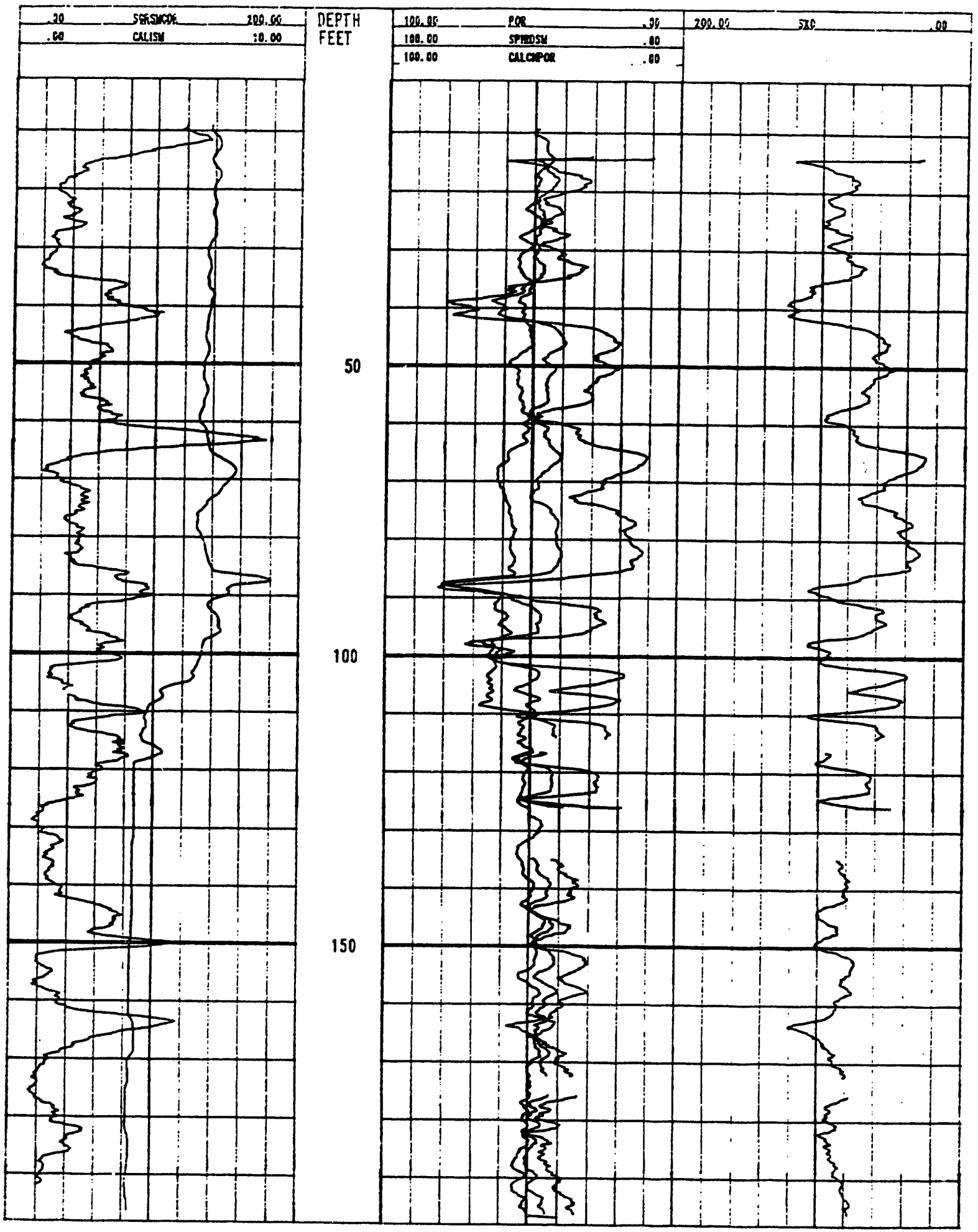

Figure VI.22. Log analysis for MHT1c, continued. POR is the total porosity with no clay correction, SPHIDSM is the stripped, smoothed density porosity, CALCNPOR is the borehole corrected neutron porosity, and $S_{x 0}$ is the water saturation in the invaded zone without the clay correction. 


\section{SIMULATION STUDIES}

Although a number of runs were made with the NUFT code to simulate the removal of VOCs and the breakthrough of helium in the helium tracer test conducted by injecting a pulse of helium into AMH-1 and measuring its concentration in AMH-2, no completely satisfactory results were achieved because we did not have the necessary geologic information until a few months from the end of the funding period. By the time we had developed the detailed description and investigated several methods for assigning permeabilities to different lithologic units to attempt generating a reasonable approximation to the heterogeneity of the system, there was not enough time to complete all the planned simulation runs and analyze the results.

As an example of the preliminary results, Fig. VII.1 was prepared, based on assigning permeabilities to each of the four lithologic types defined on the coarse-grid stratigraphic section A-A' given in Section VI. These were chosen to give a mean value of $\mathbf{k}$ equal to the value determined from the VE pressure test analysis given in Section $V$, with $\sigma$ assigned the values shown in the legend.

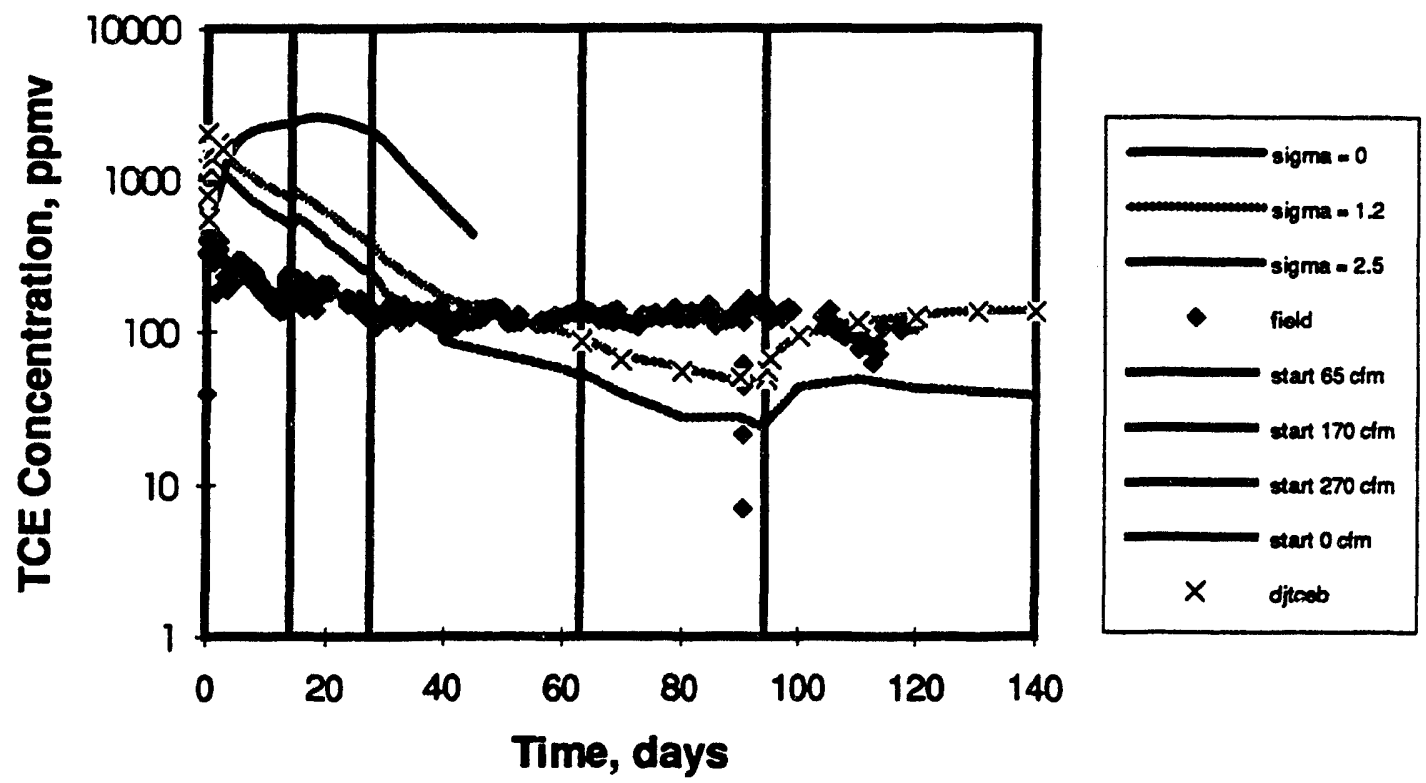

\section{Figure VII.1. TCE concentrations in AMH-2 from field data and from three simulations with the NUFT code for $\sigma$ equal to 0 (homogeneous), 1.2, and 2.5.}

Note that the performance improves as the degree of heterogeneity increases. This is an artifact of the use of a two-dimensional cross-sectional model. As $\sigma$ increases, the system becomes effectively isolated from the atmosphere because the sandy mud forms a continuous barrier near the top of the model cross-section. In reality this would not be continuous. A second problem is that we used the field contaminant concentration vs. depth data from one well to assign concentrations to all grid blocks, so that the initial contaminant distribution was not consistent with the lithology assigned. We had planned to simulate the percolation of contaminant through the system to generate a consistent set of initial conditions for each assigned permeability distribution, but this was not possible due to the termination of funding. This would be an interesting follow up study for someone to pursue. 


\section{CONCLUSIONS AND RECOMMENDATIONS}

In closing, we would first like to emphasize that this is a progress report on quantifying the effects of field-scale heterogeneities on remediation processes. It is not a finished product, and much work remains to be done even with Savannah River data before taking the results too seriously, especially on the use of the simple analytical heterogeneity parameter model to evaluate the performance of P\&T and ISAS processes at Savannah River.

However, the general approach has worked well when applied to waterflooding reservoirs with enough core data to determine accurate a priori estimates for the model parameters (pore volumes, saturations. and the variance of intrinsic permeability), and with a flood pattern and operating conditions held constant over most of the project life. In such cases, there is little difference between the parameter values determined from core data and the values obtained by least-squares fitting of the production response curves. Each waterflood response.curve requires only a single set of parameters.

Unfortunately, the Savannah River remediation projects considered in this report do not satisfy the first of these conditions. Core data are sparse and not systematically distributed, lab measurements on core samples did not include all parameters of interest for each sample, and the sampling and measurement errors in determining contaminant concentrations introduce a much larger percentage error in the contaminant mass inventory than would be typical for initial oil in place in a waterflood project.

For the P\&T project, the second condition is satisfied, and hence one major source of uncertainty in the applicability of the heterogeneity model is removed. The reasonably close agreement between the observed concentrations and model results extrapolated for six years with parameters determined from the first thirteen months of operation lends some credibility for this application.

In contrast, there were a number of significant changes in operating variables for the ISAS demonstration. In the curve-fitting exercise described in Section IV and Appendix C, it was necessary, conceptually, to associate two different pore volumes with each contaminant concentration vs. time curve in order to match the model to the field data. An analogous situation sometimes occurs in highly stratified petroleum reservoirs, when the system responds as two distinct layers connected only through the wellbores. It is then easy to justify the addition of two response curves to obtain the total production.

In the present case, this justification is not so easy. While the introduction of a mass source into the system by injecting air will certainly change the flow patterns, the treatment of the subsequent behavior as a continuation of the early time performance with a second response added is basically an ad hoc assumption and needs to be investigated further. Simulators will be very useful in this investigation. because it is then possible to specify exactly the distribution of the important physical variables. in contrast to a real system.

For now, it is interesting to speculate on the meaning of the results obtained. One of the difficult problems in analyzing the Savannah River experience is the separation of the effects of the horizontal well geometn. from the effects of air injection on the overall VOC recovery performance. Until we can develop a clear rationale for this separation. it is difficult to transfer the technology: Another problem arises in comparing in-situ air stripping with other remediation technologies which have been applied at the site. The pump and treat system has been in operation for more than seren years. but it encompasses a much larger area. For this reason. it would be expected to perform more poorly than a small-scale system located near a "hot spot" of contamination. The vertical-well vacuum extraction test conducted before the horizontal sistem was dereloped was also in the hot spot area, but the test was much too shor to allow a good comparison to be made with in-situ air stripping.

If the model results can be taken seriously, the short- and long-term performance with and without air injection can be determined directly from the two parts of the model calculation. The model result for total VOC extracted during the entire test is $1683+\mathrm{lb}$.. of which $5501 \mathrm{lb}$. is attributed to racuum extraction alone and $11333 \mathrm{lb}$. is attributed to the increase resulting from air injection. This represents a $200 \%$ 
increase in the amount of contaminant over the quantity that could have been removed in the same operating period by extraction alone from horizontal well AMH-2. From the model results, we can also correct approximately for the effects of air injection during the first 21 days and compare the results with the vertical-well SVE test. The amount of TCE attributed to extraction alone from AMH-2 is $1290 \mathrm{lb}$., and the amount of PCE is $1271 \mathrm{lb}$., giving a total of $2561 \mathrm{lb}$, or $12.5 \mathrm{lb}$. per foot of active screen. Recall from Table I.3 that the SVE test extracted $16.1 \mathrm{lb}$./ft, or approximately 1.29 times the normalized amount extracted from AMH-1. On this basis, vertical wells appear to be more efficient, but this is not really significantly different from the horizontal well results, considering the short operating time and highly variable operation of the SVE test.

It is worth noting that at least the qualitative effects of heterogeneity are captured by this simple model, in that very long time periods will be required to achieve regulatory compliance through continued extraction and air injection. Table VIII.1. shows this dramatically, with the predicted percentage of mass recovered (according to the model) increasing very slowly with continued operating time. Concentrations of contaminants in the extracted air are predicted to decrease very slowly, with more than 100 years required to reduce TCE to less than 10 parts per billion in the vapor. In Section IV, however, we noted that aqueous phase concentrations were predicted to remain above $100 \mathrm{ppb}$ even after 500 years of operation of the P\&T system.

Table VIII.1. Extrapolated remediation performance based on the heterogeneity parameter model.

\begin{tabular}{|ccccc|}
\hline $\begin{array}{c}\text { Years of } \\
\text { Operation }\end{array}$ & $\begin{array}{c}\text { Per Cent TCE } \\
\text { Removed }\end{array}$ & $\begin{array}{c}\text { Concentration } \\
(\mathbf{p p b})\end{array}$ & $\begin{array}{c}\text { Per Cent PCE } \\
\text { Removed }\end{array}$ & $\begin{array}{c}\text { Concentration } \\
(\mathrm{ppb})\end{array}$ \\
\hline 1 & 59.4 & 56610 & 51.6 & 142296 \\
10 & 90.7 & 988 & 97.6 & 1927 \\
100 & 98.1 & 30 & $>99.9$ & 2 \\
150 & $>99.0$ & 9 & $>99.9$ & $<1$ \\
\hline
\end{tabular}

Actually, the removal of contaminar : in the vapor phase may offer a considerable advantage over P\&T, whether accomplished by extraction alone, by extraction combined with injection, or either or both processes with horizontal or vertical wells. If we assume that the desired end result is a concentration of 5 $\mathrm{ppb}$ in the vadose zone aqueous phase for both TCE and PCE, then the required vapor phase concentrations (assuming equilibrium) would be 0.4 and $0.8 \mathrm{ppm}$, respectively ${ }^{1}$. We extrapolated the model results for the ISAS test to produce the plots on Fig. VIII.1, in order to estimate how long it might take to reduce the vapor concentrations to these limits. Based on these extrapolations. the vadose zone water contaminant concentrations should reach $5 \mathrm{ppb}$ after about 200,000 hours, or 23 years. of operation. This is a dramatic improvement over P\&T, but we hasten to point out that the underlying aquifer may not be cleaned up, and that the P\&T area is on the order of 100 times the size of the ISAS demonstration. The determination of the effective area (actually; sediment volume) that can be effectively treated zemains an unsolved problem.

Finally: it should be pointed out that the heterogeneity parameter approach. if validated by future work. very neatly separates the effects of heterogeneitr from the question of uncertaintr; and lends itself to rapid Monte Carlo or other probabilistic modeling techniques to determine the range of economic uncertainty arising from uncertainties in $\sigma$. total contaminant inventor; and other parameters. This in turn will ultimately allow intelligent decisions to be made concerning the allocation of resources to environmental

\footnotetext{
$1_{L}$ sing partition coefficients based on solubilities of $1100 \mathrm{mg} / \mathrm{L}$ and $150 \mathrm{mg}$ ' $\mathrm{L}$. and vapor pressures of $69.1 \mathrm{~mm} \mathrm{Hg}$ and $18.1 \mathrm{~mm} \mathrm{Hg}$. for TCE and PCE. respectivel:
} 
remediation projects. The long period required for remediation is a consequence of heterogeneity; not uncertaintr. The only uncertaintry is in how serious the problem will be.

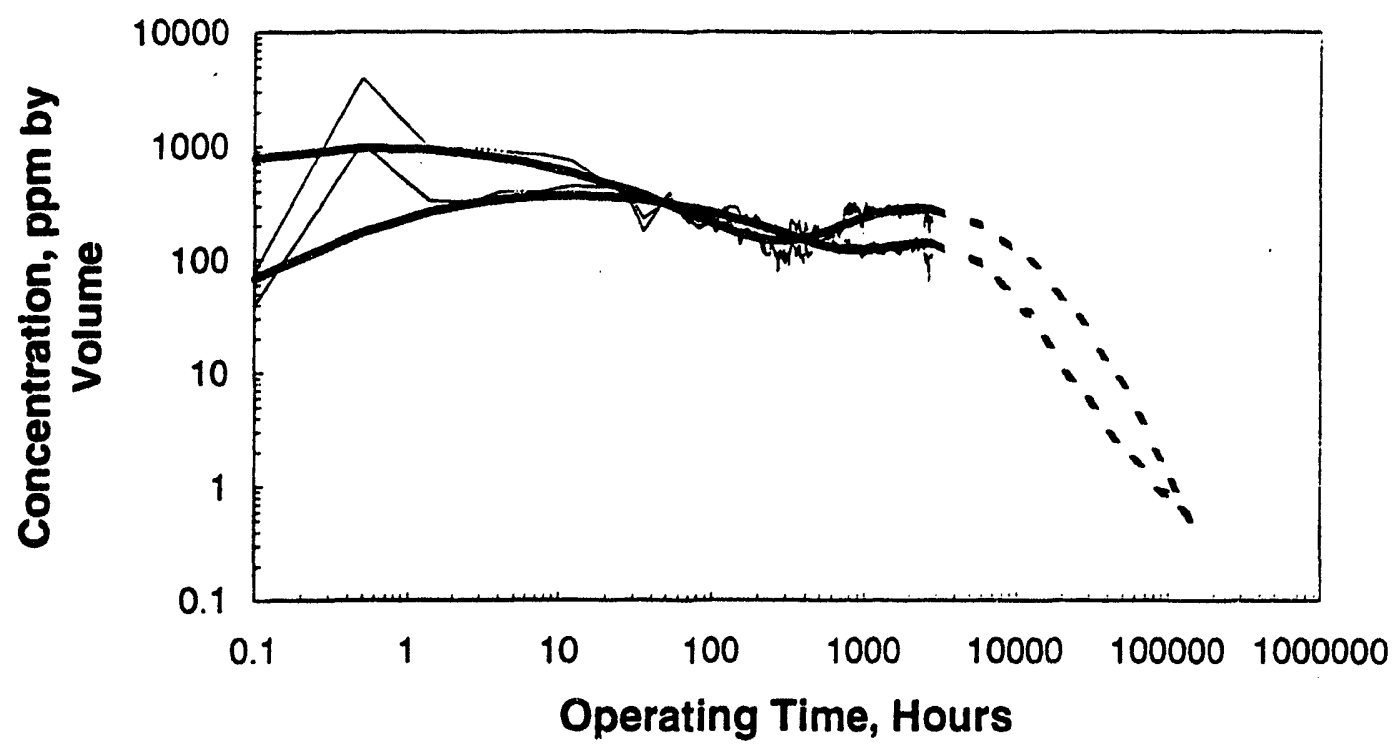

Figure VIII.1. Field data and heterogeneity model results for the ISAS test period, and extrapolated model results for the concentration of TCE and PCE versus operating time. PCE concentration is above TCE concentration for most of the time shown. The vapor phase concentrations in equilibrium with vadose zone water containing 5 ppb of each contaminant are between 0.1 and $1.0 \mathrm{ppm}$. 


\section{ACKNOWLEDGMENTS}

The authors gratefully acknowledge the support and interest of Jesse L. Yow. We wish to thank Dawn Kaback, Brian Looney, and others at the Savannah River Site for many helpful discussions and for supplying data on the Savannah River Integrated Demonstration Project, and are particularly indebted to Terry Walton and John Steele of Westinghouse Savannah River Corporation for their patience in adjusting to numerous delays.

Financial support from the U.S. Department of Energy Office of Technology Development is also gratefully acknowledged. This work was performed at Lawrence Livermore National Laboratory, Lawrence Berkeley Laboratory, and Clemson University under the auspices of the U.S. Department of Energy Office of Technology Development Technical Task Plan SF-211101, contract number W-7405ENG-48. 


\section{REFERENCES}

BISHOP, D., D. Rice, L Rogers, and P. Webster-Scholten, 1990. Comparison of Field-Based Distribution Coefficients $\left(K_{d} s\right)$ and Retardation Factors $(R s)$ to Laboratory and Other Determinations of $K_{d}$. UCRL-AR-105002, Lawrence Livermore National Laboratory, Livermore, CA.

BRAND, C.W., J.E. Heinemann, and K. Aziz, 1991. "The Grid Orientation Effect in Reservoir Simulation," SPE 21228, presented at Society of Petroleum Engineers Eleventh Symposium on Reservoir Simulation, February, Anaheim, CA.

BRIGHAM, William E., and Abbaszadeh-Dehghani, M., 1987. "Tracer Testing for Reservoir Description," Journal of Petroleum Technology, pp. 519 - 527 (May).

BIJSCHECK, Thomas A., and John J Nitao, 1992. Feasibility of In Situ Stripping of Volatile Organic Compounds at the Savannah River Site: Preliminary Modeling of a Pair of Horizontal Wells. URCRL-LR-104540, Lawrence Livermore National Laboratory, Livermore, CA.

CHESNUT, D.A., 1992. "Characterizing the Altered Zone at Yucca Mountain: the Beginning of a Testing Strategy." Proceedings, Third International High-Level Radioactive Waste Management Conference, Las Vegas, NV.

CHESNUT, D.A., D.O. Cox, and G. Lasaki, 1979. "A Practical Method for Waterflood Performance Prediction and Evaluation." Proceedings, Pan American Congress of Petroleum Engineering, Mexico City.

COATS, K.H., 1982. "Reservoir Simulation: State of the Art," Journal of Petroleum Technology, August, pp. $1633-42$.

COATS, K.H., W.D. George, C. Chu, and B.E. Marcum, 1974. "Three-Dimensional Simulation of Steamflooding," Society of Petroleum Engineers Journal, October, pp. 573-92.

COATS, K.H. and A.B. Ramesh, 1982. "Effects of Grid Type and Difference Scheme on Pattern Steamflood Simulation Results," SPE-11079, presented at 57th Annual Fall Technical Conference and Exhibition of the Society of Petroleum Engineers, September, New Orleans, LA.

COLVEN, W P., LF. Boone, J.G. Horvath, and R. Lorenz, 1987. Effectiveness of the M-Area GroundWater Remedial Action Program. DPSP-87-26, E.I. Du Pont de Nemours and Company, Savannah River Plant, Aiken SC 29808.

EARLOUGHER, R.C. JR. 1977. Advances in Well Test Analysis, Moncgraph Series 5, Society of Petroleum Engineers of AIME, Dallas

ECONOMIDES, M.J., Franz X. Deimbacher, Clemens W. Brand, and Zoltan E. Heineman, 1991. "Comprehensive Simulation of Horizontal-Well Performance," SPE Formation Evaluation 6, No. 4, Society of Petroleum Engineers.

EDDY, C.A., B.B. Looney, J.M. Dougherty, T.C. Hazen, and D.S. Kaback, 1991. Characterization of the Geology, Geochemistry, Hydrology and Microbiology of the In-Situ Air Stripping Demonstration Site at the Savannah River Site (U). WSRC-RD-91-21, Westinghouse Savannah River Company, Savannah River Site, Aiken, SC 29808.

FALLAW, W.C. and K.A. Sargent, 1986. Subsurface Geology of the $A$ and M Areas at the Savannah River Plant, Aiken, South Carolina. Contract AX 715063 for E.I. Du Pont de Nemours and Company, Savannah River Plant, Aiken SC 29808.

FALTA, R.W., I. Javandel, K. Pruess, and P.A. Witherspoon, 1989. "Density-Driven Flow of Gas in the Unsaturated Zone Due to the Evaporation of Volatile Organic Compounds," Water Resources Research 25, No. 10 , pp. 2159-2169. 
FALTA, R.W., K. Pruess, I. Javandel, and P.A. Witherspoon, 1992. "Numerical Modeling of Steam Injection for the Removal of Nonaqueous-Phase Liquids from the Subsurface. 1. Numerical Formulation," Water Resources Research 28, No. 2, pp. 433-449.

FALTA, R.W. and K. Pruess, 1991. STMVOC User's Guide, LBL-30758, Lawrence Berkeley Laboratory, Berkeley, CA.

FALTA, R.W., K. Pruess, I. Javandel, and P.A. Witherspoon, 1992. "Numerical Modeling of Steam Injection for the Removal of Nonaqueous-Phase Liquids from the Subsurface. 2. Code Validation and Application," Water Resources Research 28, No. 2, pp. 451-65.

FALTA, RONALD W., Karsten Pruess, and Dwayne A. Chesnut, 1992. "Analytical Modeling of Advective Contaminant Transport During Soil Vapor Extraction," Unpublished Presentation at AIChE Summer National Meeting, Advances in Soil Venting, Session 54, Minneapolis, MN.

FREEZE, R. ALLAN, Joel Massman, Leslie Smith, Tony Sperling, and Bruce James, 1990. "Hydrogeological Decision Analysis: 1. A Framework" Ground Water 28, No. 5, Sep. - Oct., pp. 738-766.

FREEZE, R. ALLAN, Bruce James, Joel Massman, Tony Sperling, and Leslie Smith, 1992. "Hydrogeological Decision Analysis: 4. Data Worth and the Development of Site Investigation Strategies." Ground Water 30.

KABACK, D.S., B.B. Looney, J.C. Corey, and L.M. Wright, II (1989a). Well Completion Report on Installation of Horizontal Wells for In Situ Remediation Tests, WSRC-RP-89-784, Westinghouse Savannah River Company, Savannah River Site, Aiken, SC 29808.

KABACK, D.S., B.B. Looney, J.C. Corey, L.M. Wright, III and J.L. Steele (1989b). Horizontal Wells for In-Situ Remediation of Groundwater and Soils, Westinghouse Savannah River Company, Savannah River Site, Aiken, SC 29808.

LOONEY, B.B., T.C. Hazen, D.S. Kaback, and C.A. Eddy, 1991b. Full Scale Field Test of the In-Situ Air Stripping Process at the Savannah River Integrated Demonstration Test Site (U). WSRC-RD-91-22, Westinghouse Savannah River Company, Savannah River Site, Aiken, SC 29808.

LOONEY, B.B., J.B. Pickett, and J.J. Malot, 1991a. Pilot Test of a Vacuum Extraction System for Environmental Remediation of Chlorinated Solvents at the Savannah River Site (U). WSRC-RD-91-19, Westinghouse Savannah River Company, Savannah River Laboratory, Aiken, SC 29808.

MASSMAN, JOEL, R. Allan Freeze, Leslie Smith, Tony Sperling, and Bruce James, 1991. "Hydrogeological Decision Analysis: 2. Applications to Ground-Water Contamination." Ground Water 29, No. 4, Jul. - Aug., pp. 536-548.

MATTHEWS, C.S., and D.G. Russell, 1967. Pressure Buildup and Flow Tests in Wells, Monograph Series 1, Society of Petroleum Engineers of AlME, Dallas.

PARKER, J.C., R.J. Lenhard, and T. Kuppusamy, 1987. "A Parametric Model for the Constitutive Properties Governing Multiphase Flow in Porous Media," Water Resources Research 23, No. 4, pp. 618624.

PRUESS, K., 1987. TOUGH User's Guide, NUREG/CR-4645, U.S. Nuclear Regulatory Commission, Washington, DC. (also available as LBL-20700, Lawrence Berkeley Laboratory, Berkeley, CA).

PRUESS, K., 1991. TOUGH2 - A General-Purpose Numerical Simulator for Multiphase Fluid and Heat Flow, LBL-29400, Lawrence Berkeley Laboratory, Berkeley, CA.

PRUESS, K., 1991. "Grid Orientation and Capillary Pressure Effects in the Simulation of Water Injection into Depleted Vapor Zones," Geothermics 20, No. 5/6, pp257-277.

PRUESS, K. and G.S. Bodvarsson, 1983. "A Seven-Point Finite Difference Method for Improved Grid Orientation Performance in Pattem Steam Floods," SPE-12252, Seventh Society of Petroleum Engineers Symposium on Reservoir Simulation, San Francisco, CA. 
SPERLING, TONY, R. Allan Freeze, Joel Massman, Leslie Smith, and Bruce James (1991). "Hydrogeological Decision Analysis: 3. Application to Design of a Groundwater Control System at an Open Pit Mine." Ground Water 29.

STONE, H.L., 1970. "Probability Model for Estimating Three-Phase Relative Permeability," Transactions SPE of AIME 249, pp. 214-18.

TIMUR, A. (1968). "An Investigation of Permeability, Porosity, and Residual Water Saturation Relationships." Proceedings of the SPWLA Ninth Annual Logging Symposium, June 23-26.

TODD, M.R., P.M. O'Dell, and G.J. Hirasaki, 1972. "Methods for Increased Accuracy in Numerical Reservoir Simulators," Society of Petroleum Engineers Journal, December, pp. 515-530.

TSANG, Y.W. and K. Pruess, 1989. Preliminary Studies of Gas Phase Flow Effects and Moisture Migration at Yucca Mountain, LBL-28819, Lawrence Berkeley Laboratory, Berkeley, CA.

WEBER, KJ. and L.C. van Geuns, 1990. "Framework for Constructing Clastic Reservoir Simulation Models," Journal of Petroleum Technology, October, Society of Petroleum Engineers.

YANOSIK, J.L. and T.A. McCracken, 1979. "A Nine-Point, Finite Difference Reservoir Simulator for Realistic Prediction of Adverse Mobility Ratio Displacements," Society of Petroleum Engineers Joumal, August, pp. 253-62. 


\section{APPENDIX A. STREAMLINE MOdels FOR Vertical VAPOR EXTRACTION WELLS AND HORIZONTAL INJECTION AND EXTRACTION WELLS}

This Appendix comprises two separate documents, reproduced here as completed in August 1992 except for changes in the cover pages and page numbering to improve consistency with the remainder of this report. Document 1, Analytical Modeling of Advective Contaminant Transport During Soil Vapor Extraction, was co-authored by Ronald W. Falta (Clemson University), Karsten Pruess (Lawrence Berkeley Laboratory), and Dwayne A. Chesnut (Lawrence Livermore National Laboratory), to document a portion of work performed for the Analysis and Evaluation Task on the Savannah River Integrated Demonstration Project, under LLNL Technical Task Plan SF-211101 for the Office of Technology Development, DOE. This report was submitted to the American Institute of Chemical Engineers as a preprint for the 1992 Summer National Meeting (Advances in Soil Venting, Session 54) in Minneapolis, $\mathrm{MN}$, and presented orally at the meeting by the senior author.

Document 2, Analytical Solutions for Gas Flow Due to Gas Injection and Extraction from Horizontal Wells, was written by Ronald W. Falta (Clemson University) in partial fulfillment of a subcontract to Lawrence Berkeley Laboratory under LLNL Technical Task Plan SF-211101. It is also reproduced here as received, except for changes in format of the first page. Page numbering starts with the first integer after the last page of document 1 . 


\title{
analytical modelng of advective Contammati $r$ Transport DURING SOIL VAPOR EXTRaction
}

\author{
Ronald W. Falta \\ Department of Earth Sciences, Clemson University, Clemson, SC \\ Karsten Pruess \\ Earth Sciences Division, Lawrence Berkeley Laboratory, Berkeley, CA \\ Dwayne A. Chesnut \\ Earth Sciences Division, Lawrence Livermore National Laboratory, Livermore, CA
}

August 12, 1992

\begin{abstract}
A general method for modeling the advective transport of a Volatile Organic Compound (VOC) to a soil vapor extraction well has been developed. This technique is applicable to cases in which the VOC is partitioned in the unsaturated zone between the gas, aqueous, and solid phases. The VOC may have a one, two, or three-dimensional initial distribution. The method may be applied to problems having a one or two-dimensional steady state gas flow field in which the gas streamfunction and gas travel time distributions are known. Examples are included to show the application of the method to radial and twodimensional cylindrical gas flow fields with different VOC distributions and characteristics.
\end{abstract}




\section{INTRODUCTION}

Soil vapor extraction is a new method for removing volatile organic compounds (VOCs) from the unsaturated zone. A soil vapor extraction system consists of one or more gas pumping wells screened in the unsaturated zone. The induced gas flow results in the evaporation of nonaqueous phase liquids (NAPLs), the volatilization of chemicals dissolved in residual pore water, and the desorption of chemicals from soil surfaces. Contaminant removal is achieved as the gas sweeps the contaminated zone, flushing contaminant vapors from the soil.

In the design and evaluation of vapor extraction systems, mathematical models of the gas flow field and contaminant transport are useful tools. Usually, these models are based on numerical solutions of the governing mass balance equations, and a number of numerical simulators have been developed for modeling gas flow and chemical transport in the unsaturated zone $[1.2,3,4,5,6,7,3]$.

For certain well geometries and system conditions, it is possible to develop analytical solutions for modeling the induced gas pressure field. Analytical pressure solutions have been presented for one-dimensional radial flow $[9,10,11,12,13]$ and for two-dimensional cylindrical flow $[14,15.16]$ under various conditions.

These gas pressure solutions may be used to calculate the gas travel time from any location to the extraction well (see, for example, $[11,14,16])$. Shan et al. [16] showed that the gas streamfunction distribution may be analytically derived from the pressure solution.

While several analytical solutions are available for modeling the gas pressure field under different conditions, the number of analytical solutions reported for modeling 
chemical transport during soil vapor extraction operations is more limited. For problems in which the contaminant is present in the form of a pool of NAPL resting on the water table or trapped in a low permeability soil layer, Johnson et al. [9], Johnson et al. [11,12], and Ho and Udell [17] have presented boundary layer type solutions for the chemical transport assuming one-dimensional gas flow. In these solutions, the gas is assumed to flow past but not through the contaminated zone, and the rate of contaminant removal is limited by gaseous diffusion from the contaminated zone into the flowing gas stream.

An alternate scenario is one in which the VOC is not present in the form of a NAPL, and in which the gas flows through the contaminated zone. In this case, the effect of diffusion is usually much smaller, and the rate of contaminant removal is mainly limited by the rate of gas flow through the contaminated zone. For situations in which the VOC is strongly partitioned into the aqueous and solid phases, the rate of local interphase mass transfer into the gas phase could also limit the rate of contaminant removal.

In this paper, we present a general method for modeling the advective transport of a VOC to a soil vapor extraction well. This technique is applicable to cases in which the VOC is partitioned in the unsaturated zone between the gas, aqueous, and solid phases, and in which the assumption of local phase equilibrium is valid. The VOC may have a one, two, or three-dimensional initial concentration distribution, and the method may be applied to problems having a one or two-dimensional steady state gas flow field in which the gas streamfunction and gas travel time distributions are known. Examples are included to show the application of the method to radial and two-dimensional 
cylindrical gas flow fields with different initial VOC characteristics and distributions.

This technique may be used to optimize the placement of a vapor extraction well screen for a given subsurface VOC distribution, and is useful for conducting initial feasibility studies of vapor extraction as a remediation method. This analytical method may also be used to verify the performance of more sophisticated numerical models used to simulate gas phase contaminant transport.

\section{METHODOLOGY AND ASSUMPTIONS}

The basis of the method is a mapping of the initial contaminant distribution from the $r-z$ or $x-z$ plane to a vapor travel time-normalized streamfunction plane. In constructing this mapping, the normalized streamfunction (fractional mass or molar flow) is usuaily determined analytically by applying the Cauchy-Riemann equations to an analytical steady state pressure distribution solution (see, for example, [16]). For simple flow systems, the vapor travel time may also be determined analytically from the pressure solution, and fc: more complicated systems, the travel time distribution may be computed numerically from the pressure solution.

From the transformed chemical distribution, the fraction of contaminated soil gas entering an extraction well at any time may be found directly. This fraction determines the composition of the gas pumped from the well, and by using this information with the known gas flowrate from the weil. the rate of contaminant removal may be calculated.

The technique described above is similar in many respects to approaches used to model advective transport in sarurated fluid flow. For example, Javandel et al. [18] 
developed a semi-analytical method for computing advective contaminant transport in two-dimensional horizontal fluid flow systems. They use complex potential theory to determine streamfunction distributions, and calculate travel times numerically from the complex velocity potential. This information is then used to compute contaminant breakthrough curves at pumping wells, and to determine advective front locations.

In the present work, we are primarily interested in finding the contaminant concentration produced from a vapor extraction well as a function of time. By formulating the advective modeling method as a mapping problem, this calculation is done in a general way.

To use steady state gas streamfunction and travel time solutions to model advective VOC transport, several simplifying assumptions are necessary. A primary requirement is that the movement of VOC from a given location must be proportional to the gas pore velocity at that location. This requirement is satisfied under conditions of linear equilibrium partioning of the contaminant between the gas, aqueous, and solid phases. In this case, the VOC advective velocity is related to the soil gas pore velocity by

$$
V_{c}=\frac{V_{g}}{O S_{g} R_{g}}
$$

where $v_{e}$ is the VOC advective velocity, $v_{g}$ is the soil gas darcy velocity, $\phi$ is the porosity, $S_{g}$ is the gas phase saturation. and $R_{g}$ is the gas phase retardation coefficient for the specific chemical. With linear equilibrium phase partioning, $R_{g}$ is defined as [19]

$$
R_{q}=\frac{S_{w}}{H S_{q}}+\frac{\rho_{b} K_{D}}{H \Phi S_{g}}+1
$$


where $S_{w}$ is the residual aqueous phase saturation, $p_{b}$ is the soil bulk density, $K_{D}$ is the chemical-soil distribution coefficient, and $H$ is the chemical's Henry's constant. The chemical-soil distribution coefficient is often estimated by

$$
K_{D}=K_{o c} f_{o c}
$$

where $K_{\alpha c}$ is the chemical-organic carbon partition coefficient, and $f_{\alpha c}$ is the fraction of organic carbon in the soil $[20.21]$. Methods for calculating $K_{\infty c}$ for different chemicals are given by Lyman et al. [22]. As used in equation (2), Henry's constant is dimensionless, and may be approximately calculated from

$$
H=\frac{\bar{C}_{q}^{c}}{\bar{C}_{w}^{c}}
$$

where $\bar{C}_{g}^{c}$ is the saturated vapor concentration of the chemical, and $\bar{C}_{w}^{c}$ is the aqueous solubility of the chemical. The use of equations (1) and (2) for determining the VOC transport require that the VOC is not present as a NAPL in the system. In the present work, we will assume that the $R_{\mathfrak{g}}$ is constant throughout the subsurface. This implies that $S_{w}, K_{D}, \phi$, and $\rho_{b}$ are constant and uniform throughout the system.

Because steady state pressure and streamfunction solutions are used to model the gas transport, changes in the How pattern due to changes in the gas composition are neglected. When the gas phase concentration of a VOC is large, significant gas flows may occur due to buovancy effects [5.6.23].

This effect would be paricularly noticeable during the evaporation of high vapor pressure NAPLs, a situation winich is not considered here. At lower gas concentration levels, this phenomenon are less important. To illustrate this point consider the organic 
solvent, trichloroethylene (TCE). At a temperature of $25^{\circ} \mathrm{C}, \mathrm{TCE}$ has a vapor pressure of $9.9 \mathrm{kPa}$. At a total pressure of $1 A T M$, this corresponds to a gas phase mole fraction of 0.098 (98,000 parts per million by volume), and a vapor concentration of 0.52 $\mathrm{kg} / \mathrm{m}^{3}$. The density of soil gas with this composition would be $1.58 \mathrm{~kg} / \mathrm{m}^{3}$ which is substantially larger than the ambient soil gas density of $1.17 \mathrm{~kg} / \mathrm{m}^{3}$ [5]. On the other hand, if the TCE gas phase mole fraction is smaller, say $0.01(10,000$ PPMV), the vapor concentration would be $0.054 \mathrm{~kg} / \mathrm{m}^{3}$, and the total gas density would be 1.21 $\mathrm{kg} / \mathrm{m}^{3}$ which is close to the ambient soil gas density. Although the TCE gas concentration in this second example is only about one tenth the saturated value, it would still represent a very significant level of subsurface contamination. The equilibrium aqueous concentration corresponding to a gas concentration of $0.054 \mathrm{~kg} / \mathrm{m}^{3}$ may be calculated using Henry's law with Henry's constant, $H$ calculated from equation (4). The equilibrium aqueous concentration in this case is about $0.113 \mathrm{~kg} / \mathrm{m}^{3}$ or $113 \mathrm{mg} / \mathrm{l}$. This would be considered a high level of subsurface contamination, and remediation would probably be required.

Changes in the gas composition may also affect the characteristics of gas removal from a well. For example. if an extraction well is pumped at a constant volumetric rate, as the concentration of VOC in the gas increases, the gas density increases (for compounds having molecular weights greater than air), and the total rate of gas mass removal increases.

An additional phenomena which is neglected in the present development is the release of contaminants from the aqueous and solid phases due to the depressurization of the gas phase. As gas expands during depressurization, the VOC partial pressure 
and gas phase mass concentration decline. In order to maintain local chemical phase equilibrium, this requires a reduction in the VOC concentration in the aqueous and solid phases. Although this effect may be important under conditions of large and widespread gas depressurization, during most soil vapor extraction operations, the zone of large gas pressure change is restricted to an area very close to the extraction well (see, for example, $[11,12,16])$.

\section{APPLICATION TO RADIAL GAS FLOW TO A VAPOR EXTRACTION WELL}

The streamfunction-travei rime mapping method for modeling advective gas transport may be illustrated by application to a simple flow field. In this :tion, we consider a fully confined radial gas flow system in which the gas extraction well is screened throughout a permeable formation having uniform and constant properties.

\section{Gas Flow Field and Travel Times}

For boundary conditions consisting of a constant pressure, $P_{w}$ at a well radius of $r_{w}$ and atmospheric pressure $\left(P_{a}\right)$ at a radial distance from the well of $r_{i}$, Johnson et al. [11] solved the goveming porous media gas flow equation to obtain the steady state radial pressure distribution:

$$
\left.P=P_{w}^{2}+\frac{\left(P_{i}^{i}-P_{w}^{i}\right)}{\ln \left(r_{i} / r_{w}\right)} \ln \left(r / r_{w}\right)\right]^{1 / 2}
$$

Lising Darcy's law. they obtained the radial gas darcy velocity from equation (5)

$$
V_{g}=-\frac{i}{-! \perp} \frac{\left(\frac{P_{w}}{\left.r \ln \left(r_{w} r_{i}\right)\right)}\left(1-\left(P_{a} / P_{w}\right)^{2}\right)\right.}{\left[1+\left(1-P_{a}\left(P_{w}\right)^{2}\right) \frac{\ln \left(r / r_{w}\right)}{\ln \left(r_{w} / r_{i}\right)}\right]^{1 / 2}}
$$


where $k$ is the effective gas phase permeability, and $\mu$ is the gas viscosity. The volumetric gas flowrate entering the well may be computed using equation (6):

$$
Q_{w}=\frac{h \pi k}{\mu} \frac{P_{w}\left(1-\left(P_{a} / P_{w}\right)^{2}\right)}{\ln \left(r_{w} / r_{i}\right)}
$$

where $h$ is the thickness of the pumped formation, and $Q_{w}$ is measured at a pressure of $P_{w}$

For a system composed of ideal gases, the total molar flowrate entering the well may be computed by

$$
\bar{M}=Q_{w} C_{w}
$$

where $C_{w}$ is the molar density of gas entering the well calculated by

$$
C_{w}=\frac{P_{w}}{R T}
$$

It is useful to note that the total molar flowrate entering the well, $\vec{M}$, is independent of the gas composition entering the well for this problem. This is due to the fact that the molar density is not a function of gas composition.

Given a constant total molar flowrate distributed uniformly over the screen length, the normalized streamfunction corresponds physically to a fractional molar flowrate. That is, the molar flowrate between any two streamlines is constant, and the sum of these streamtube flowrates is equal to the total molar flowrate. For one-dimensional radial flow to a fully penetrating well. the normalized streamfunction (fractional flow) is easily computed as a linear function of depth. Considering a pumped zone of thickness $h$, the normalized streamfunction distribution may be written as: 


$$
\psi_{d}=1-z / h
$$

where $z$ is the depth from the top of the formation. Using equation (10), $\Psi_{d}$ has a maximum value of 1 at the upper confining layer and a minimum value of zero at the lower confining layer.

For this problem, the unretarded gas travel time from any radial distance to the well may be calculated using equation (6) [11]

$$
\tau=\int_{r_{\infty}}^{r} \frac{\phi S_{g}}{V_{g}\left(r^{\prime}\right)} d r^{\prime}
$$

The advective VOC travel time considering equilibrium phase partitioning may be calculated by simply multiplying $\tau$ by the gas phase retardation coefficient, $R_{8}$ given by equation (2). The advective VOC travel time, $\tau_{R}$ is then

$$
\tau_{R}=\tau R_{\xi}
$$

In Figure 1, the normalized streamfunction (horizontal lines) and VOC gas travel time (vertical lines) distributions are plotted. These distributions were calculated for a problem in which the formation thickness is $10 \mathrm{~m}$, the effective gas permeability, $k$ is $1 \times 10^{-12} \mathrm{~m}^{2}$, the porosity is 0.4 . and the residual water saturation, $S_{w}$ is 0.25 . The VOC travel times were computed by numerically integrating equation (7), and the transport properties of trichloroethylene (TCE) at $20^{\circ} \mathrm{C}$ were used to calculate $R_{g}$ used in equation (12). A listing of the problem specirications for this example are given in Table 1.

The TCE Henry's constant in Table 1 was calculated using equation (4) as the ratio of the TCE saturated vapor concentration to the TCE aqueous solubility. The saturated vapor concentration was computed using the ideal gas law [5] with the 
temperature-dependent vapor pressure given by Reid et al. [24]. The TCE solubility is assumed to be relatively independent of temperature, and a value of $1.1 \mathrm{~g} /$ was used [25]. The soil-water distribution coefficient in Table 1 was calculated by equation (3) with an assumed value of $f_{o c}$ of 0.001 , and a TCE $K_{\propto c}$ value of $0.126 \mathrm{~m}^{3} / \mathrm{kg}$ [25].

The contour interval for the VOC travel times in Figure 1 is one day; in other words, TCE vapor located along the first contour will arrive at the extraction well in one day, vapor located at the second contour arrives at the well in two days, and so forth. For one-dimensional radial flow, the travel time contours are vertical, and are a nonlinear function of the radial distance from the well. The contour interval for the normalized streamfunction in Figure 1 is 0.1 , and $\psi_{d}$ varies from a value of 1.0 at $z=0$ to 0.0 at $z=10 \mathrm{~m}$. For radial flow, the streamlines in cross-sectional view are horizontal and are a linear function of the depth from the confining layer.

\section{Conical Initial Contaminant Distribution}

To show the application of the mapping method to a radial flow problem, we will consider a conically shaped initial TCE distribution as shown in Figure 2. In this figure, the contaminated zone is represented by the hatched area, and it is assumed to have a uniform gas phase TCE mole fraction of $\chi_{0}$. The mapping of this contaminated zone from a $r, z$ coordinate system to a $\tau_{R}, \psi_{d}$ coordinate system may be performed graphically using Figure 2.

This is done by finding the $\tau_{R}$ and $u r_{d}$ values corresponding to points along the outside edges of the contaminated zone. and plotting these values on a $\tau_{R}, \psi_{d}$ graph as shown in Figure 3. The thickness of the hatched area at any value of vapor travel time 
in this figure then represents the fraction of contaminated gas entering the well at that time. For example, initially (time=0), all of the gas entering the well is contaminated because the thickness of the transformed zone at time $=0$ is 1 . Referring back to Figure 2, it is clear that this is the case, because the entire well screen is initially in contact with contaminated gas. Later, after four days of vapor extraction, the thickness of the transformed section in Figure 3 is about 0.6 , indicating that about $60 \%$ of the gas entering the well is contaminated while the remaining $40 \%$ is clean (neglecting any diffusion). This may be verified by checking the initial distribution in Figure 2. At the $\tau_{R}$ contour corresponding to 4 days, $60 \%$ of the streamtubes contain contaminated gas.

For the purpose of convenience, the thickness of thi: :ansformed section as a function of travel time may be plotted separately, and a plot of this type is shown in Figure 4. For a contaminated zone containing a TCE mole fraction of $\chi_{0}$, this curve gives the relative TCE mole fraction leaving the well, $\chi / \chi_{0}$, versus time. The mass rate of VOC removal from the subsurface at any time is calculated by

$$
i i^{\epsilon}=\chi(t) \tilde{M} M_{m}
$$

where $M_{w t}$ is the contaminant molecular weight, and $\bar{M}$ is calculated from equations (7) and (8). The cumulative VOC mass removal from the system at some time, $t^{*}$, is obtained by integrating equation (13) with respect to time,

$$
W_{c w n}^{c}=\bar{M} M_{w t} \int_{0}^{t} \chi(t) d t
$$

Although the advective transport method described in this section was based on graphical techniques, the deveiopment of computer programs for implementing this 
method is straightforward, depending on the complexity of the initial contaminant distribution.

\section{APPLICATIONS TO TWO-DIMENSIONAL (R-Z) GAS FLOW TO A VAPOR EXTRACTION WELL}

In this section, the streamfunction-travel time mapping method for modeling advective gas transport is applied to a more complex two-dimensional cylindrical gas flow field. Examples are given for several initial contaminated zone geometries, and the results for selected problems are compared with those computed using a compositional multiphase flow simulator.

\section{Gas Flow Field and Travel Times}

Referring to Figure 5, we will consider a steady-state gas flow field resulting from gas extraction from a well screened from $z=b$ to $z=a$ in the unsaturated zone. The porous media is assumed to be homogeileous, but may be anisotropic, and the well is pumped at a constant volumetric rate of $Q_{a}$. The ground surface is assumed to be open to the atmosphere $\left(P=P_{a}\right)$, and the system is assumed to be bounded by a no-flow boundary at a depth of $h$. This lower boundary would correspond to either an impermeable stratum. or the water table (neglecting any movement of the water table due to gas pumping).

The analytical solutions for the gas pressure and streamfunction distributions under these conditions are given by Shan et al. [16]. As presented in [16], the solutions apply to a case where the gas well is pumped at a constant total mass rate, $\dot{M}$, and the 
solutions are written in terms of a dimensionless source strength defined as:

$$
\xi=\frac{\mu \dot{M}}{2 \pi k_{h} P_{a} P_{a} L}
$$

where $k_{h}$ is the effective horizontal gas permeability, $\rho_{a}$ is the ambient gas density, and $L$ is the thickness of the screened interval. It is more convenient to write equation (15) in terms of a volumetric flow rate, $Q_{a}$ defined as

$$
Q_{a}=\frac{\dot{M}}{\rho_{a}}
$$

because this allows the solutions to be applied to cases in which the gas has a variable composition. Using equation (16), $Q_{0}$ is the total volumetric 太owrate leaving the well measured at ambient pressure and temperature $\left(P_{a}, T\right)$. As in the previous section, the total molar flowrate leaving the well may be computed by multiplying the volumetric flowrate by the molar density,

$$
\tilde{H}=Q_{a} C_{a}
$$

where $C_{a}$ is the gas molar density at ambient conditions,

$$
C_{a}=\frac{P_{a}}{R T}
$$

With these modifications to the Shan et al. [16] solutions, the normalized streamfunction, $\psi_{d}$ corresponds to a fractional molar flowrate, and the sum of the molar flowrates through all of the streamiubes is equal to the total molar flowrate, $\vec{M}$.

In Figure 6, the normalized streamfunction $\left(\psi_{d}\right)$ and unretarded gas travel time $(\tau)$ distributions are shown for an isotropic case in which the formation thickness, $h$ is $40 \mathrm{~m}$, and the volumetric gas extraction rate. $Q_{0}$ is $0.155 \mathrm{~m}^{3} / \mathrm{s}(328 \mathrm{cfm})$. Additional 
parameter values for this case are given in Table 2 . The $\psi_{d}$ contour interval in this figure is 0.05 , corresponding to 20 streamtubes. Each of the streamlines originate at the ground surface, and terminate at the well screen, and the molar flowrate between each streamline is constant. By convention, the top streamline, extending from $r=0, z=0$ to the well screen has a $\psi_{d}$ value of one, while the bottom streamline, extending from $r=$ $\infty, z=h$ to $r=0, z=h$, and up to the well screen has a value of zero. We note that this convention is the reverse of that used by [16].

The gas travel time $(\tau)$ distribution in Figure 6 was calculated using a simple particle tracking path integration method [16], and the travel time contour interval is one day. As in the radial flow case (Figure 1), the first contour from the well corresponds to a gas travel time of 1 day, the second contour corresponds to a travel time of 2 days, and so on. It is important to note that for this flow geometry, the gas travel times become very large as the radial distance from the well increases. This is largely due to the fact that most of the gas pumped from the well originates at the ground surface a short radial distance away from the well. With the $\psi_{d}-\tau$ distribution given by Figure 6. it is now possible to construct advective gas transport solutions for various initial contaminant distributions.

\section{Cylindrical and Conical Initial Contaminant Distributions}

Consider the cylindrically shaped contaminant distribution shown as the shaded area in Figure 7. This shaded area corresponds to a region in which the VOC gas phase mole fraction is equal to $\chi_{0}$. The transtormation of this zone into the $\psi_{d}-\tau$ plane is shown by Figure 8 . As in the radial How case presented earlier, the transformed section 
geometry was computed by finding the $\tau$ and $\psi_{d}$ values corresponding to locations along the outside edges of the initial contaminant distribution shown in Figure 7. The thickness of the transformed section at any value of $\tau$ determines the fraction of contaminated gas entering the well screen at any time, and this curve is shown by Figure 9. In Figure 9, the vertical axis corresponds to the relative mole fraction of gas entering the well, $\chi / \chi_{0}$, and the horizontal axis corresponds to the unretanded gas travel time, $\tau$. Initially, the gas entering the well consists of $50 \%$ clean gas and $50 \%$ contaminated gas. This is due to the relative locations of the well screen and the contaminated zone. From Figure 7 , it can be seen that half of the screen is located in the contaminated region.

As the well is pumped, the fraction of contaminated gas entering the well decreases as more and more of the streamtubes contain clean gas. For example, after 4 days of pumping, only about $15 \%$ of the gas entering the well is contaminated (Figure 9). In other words, after 4 days, only about 3 of the 20 streamtubes in Figure 7 are delivering contaminated gas to the well. This may be verified by checking the number of streamtubes along the 4-day travel time contour which are within the contaminated zone in Figure 7.

For a given initial VOC mole fraction in the contaminated zone, and a gas phase retardation coefficient calculated by equation (2), Figure 9 may be used to compute the time-dependent composition of the gas entering the well. This is done by simply multiplying the vertical axis by $\chi_{0}$ and the horizontal axis by $R_{g}$. This results in a plot of VOC mole fraction, $\chi$ versus retarded travel time, $\tau_{R}$, and the VOC mass rate and cumulative amount of VOC removed from the system at any time are given by (13) and 
(14), respectively.

As mentioned earlier, once the $\psi_{d}-\tau$ distribution for a vapor extraction well geometry is developed, advective gas transport solutions may be constructed for various initial contaminant zone geometries. In Figure 10, a case is shown in which the VOC is distributed in a conical volume similar to the radial flow example. The transformation of this shape into the $\psi_{d}-\tau$ plane is given in Figure 11, and the resulting thickness of the transformed shape as a function of gas travel time is shown in Figure 12. Although the VOC distribution considered in this example is similar to that shown before for the radial flow case. Figure 2. the transformed shape shown in Figure 11 is quite different from that developed for the radial flow situation, Figure 3. This is due to the more complex two-dimensional nature of the streamfunction and travel time distributions used to develop the coordinate transformation.

While the examples discussed thus far have only considered contaminated zones having a uniform VOC mole iraction. more complicated distributions may be modeled by superimposing the solutions from several different initial distributions. Furthermore, it is possible to model the advective transport resulting from a three-dimensional contaminant distribution in which the distribution is a function of $r, z$, and $\theta$. This is done by multiplying the relative mole fraction in the $\chi / \chi_{0}$ vs $\tau$ breakthrough curve by the fractional flow through the contaminated zone in the $\theta$ dimension (see $[11,17])$. For example, if the contaminated zones shown in Figures 2, 7, and 10 were assumed to only subtend and angle of $\alpha$ in the $\theta$ dimension. the breakthrough curves shown in Figures 4 , 9. and 12 would be multiplied by $\alpha / 2 \pi$. This would account for the fact that the gas flowing through the remaining angle of $2 \pi-\alpha$ is not passing through a contaminated 
area.

\section{Uniform and Layered Initial Contaminant Distributions}

Because the advective transport model described in this paper is not subject to numerical discretization errors, the method may be used to verify the accuracy of numerical models for gas phase advective transport. In this section, two examples are given in which the results calculated by the present method are compared with those obtained using a comprehensive multiphase compositional numerical simulator known as STMVOC developed by Falta et al. [7].

For the first example, we consider the two-dimensional flow field resulting from gas extraction from a well screened between $3 \mathrm{~m}$ and $7 \mathrm{~m}$ below the ground surface in a $10 \mathrm{~m}$ thick formation. The system has an anisotropic permeability, and the ground surface is open to the atmosphere. For this problem, the entire subsurface is assumed to be uniformly contaminated with an initial chemical mole fraction of $x_{0}=0.01$. The air entering from the ground surtace is assumed to be clean, and phase partitioning of the chemical is neglected $\left(R_{g}=1\right)$. Additional problem specifications are listed in Table 3 .

This problem was modeled using STMVOC with a very fine mesh. A total of 1050 elements were used with 20 uniformly spaced elements in the vertical dimension and 50 logarithmically spaced elements in the radial elements. The remaining 50 elements were placed at the top of the mesh. and were used to maintain the upper boundary condition of zero contaminant mole fraction and constant atmospheric gas pressure. In order to match the assumptions used in the analytic model (ie. steady state flow), an initial computer run was conducted to generate the steady state pressure. This 
pressure field, and a gas phase chemical mole fraction of 0.01 were then used as initial conditions for the transport simulation.

A comparison of the numerical results with the solution obtained using the analytical method is shown in Figure 13. In this figure, the solid line represents the analytical solution and the solid circles represent the numerical solution result at every time-step. These results are in good agreement, and one could conclude that the numerical simulator is giving accurate results for this particular flow and contaminant geometry.

The final example is somewhat more realistic, consisting of a layered initial trichloroethylene distribution with equilibrium phase partitioning. In this case, gas is pumped from the bottom $3 \mathrm{~m}$ of a $10 \mathrm{~m}$ thick anisotropic formation which is open to the atmosphere at the ground surface. The initial TCE distribution consists of 6 horizontal layers with a uniform TCE mole fraction in each layer. Details of the initial TCE distribution, along with additional parameters used in this problem are listed in Table 4.

This case was modeled numerically with STMVOC using the same mesh as in the last problem. Because this problem includes some pore water, and evaporation of pore water is not considered in the analytic method, the STMVOC code was slightly modified to eliminate water evaporation in this simulation. In the numerical model, phase partitioning is included by assuming local chemical equilibrium in each element [7]. As in the previous exampie. an initial run was conducted to generate the steady state gas pressure field. This gas pressure field was then used with the initial TCE distribution specified in Table 4 to form initial conditions for the numerical transport simulation. 
To generate the analytical solution for this case, the transformed solution for each layer was computed individually. These were then superimposed to give the overall contaminant breakthrough curve. A comparison of this curve with the numerical simulation results is given in Figure 14. Initially, the TCE mole fraction entering the well is zero due to the fact that the well screen is located in an area which is initially free of TCE. After a short time, the TCE mole fraction begins to increase as gas from a highly contaminated zone just above the well screen enters the well. A maximum mole fraction is reached after about 60,000 s (about 0.7 days) of pumping, and following this peak, the mole fraction entering the well begins to decrease as gas from the less highly contaminated layers enters the well. The numerical results, shown as solid circles in Figure 14, are in good agreement with the analytical solution, although the value of the peak mole fraction entering the well is slightly underestimated by the code.

\section{SUMMARY}

A relatively simple analytical technique has been developed for simulating the advective transport of VOCs in the unsaturated zone in response to soil vapor extraction operations. This method is based on a coordinate transformation defined by the gas streamfunction and travel time distributions for a given vapor extraction well geometry. The method may be applied to one and two-dimensional flow fields with complex initial VOC distributions, and the effects of equilibrium phase partitioning of the VOC into residual pore water and the solid phase are accounted for.

This method may be used in soil vapor extraction optimization studies, and it provides a very efficient means for conducting initial feasibility studies at hazardous waste 
sites requiring remediation. These solutions may also be used to verify the performance of numerical simulators developed for gas phase contaminant transport modeling.

\section{NOTATION}

a depth to well screen bottom, $m$.

$b \quad$ depth to well screen top, $m$.

$C_{0} \quad$ gas molar density at ambient pressure and temperature, moie $/ \mathrm{m}^{3}$.

$C_{w} \quad$ gas molar density at weil screen pressure and temperature, mole $/ \mathrm{m}^{2}$.

$\bar{C}_{\xi}^{c} \quad$ saturated VOC vapor concentration, $\mathrm{kg} / \mathrm{m}^{3}$.

$\bar{C}_{w}^{*} \quad$ VOC aqueous solubility, $\mathrm{kg} / \mathrm{m}^{2}$.

$f_{o c} \quad$ fraction of organic carbon in soil.

h formation thickness. $m$.

H Henry's Constant detined by equation (4).

$i \quad$ effective gas phase permeability, $m^{2}$.

$k_{h} \quad$ effective horizontal gas phase permeability, $m^{2}$. 
$k_{v} \quad$ effective vertical gas phase permeability, $m^{2}$.

$K_{D} \quad$ soil-water distribution coefficient, $m^{3} / \mathrm{kg}$.

$K_{\infty} \quad$ organic carbon partition coefficient, $m^{3} / k_{g}$.

$L \quad$ length of screened interval, $m$.

$\dot{M} \quad$ total mass flowrate from well, $\mathrm{kg} / \mathrm{s}$.

$\dot{M}^{c} \quad$ total VOC mass flowrate from well, $\mathrm{kg} / \mathrm{s}$.

$\bar{M} \quad$ total molar flowrate from well, mole/s.

$M_{\text {cwn }}^{c} \quad$ cumulative VOC mass removed, $\mathrm{kg}$.

. $_{\mathrm{w}} \quad$ VOC molecular weight, g/mole.

$P \quad$ gas pressure, $P a$.

$P_{a} \quad$ ambient gas pressure. $P a$.

$P_{w} \quad \cdot$ well screen gas pressure. $P a$.

$Q_{a} \quad$ total volumetric flowrate at ambient pressure and temperature, $\mathrm{m}^{3} / \mathrm{s}$.

$Q_{w} \quad$ total volumetric flowrate at well screen pressure and temperature, $\mathrm{m}^{3} / \mathrm{s}$.

$r_{i} \quad$ radius of influence. $m$.

$r_{w} \quad$ well radius, $m$.

$R \quad$ universal gas constant. $m J /$ mole $K$.

$R_{g} \quad$ gas phase retardation coefficient.

$S_{q} \quad$ volumetric gas phase saturation.

Sw volumetric aqueous phase saturation. 
$T \quad$ temperature, ${ }^{\circ} \mathrm{C}$.

$V_{c} \quad$ VOC advective velocity, $\mathrm{m} / \mathrm{s}$.

$V_{8} \quad$ gas phase darcy velocity, $\mathrm{m} / \mathrm{s}$.

$\alpha \quad$ angle subtended by contaminated zone, radians.

$\chi \quad$ VOC gas phase mole fraction.

$\chi_{0} \quad$ initial VOC gas phase mole fraction.

porosity.

$\mu \quad$ gas phase viscosity, $\mathrm{kg} / \mathrm{m} \mathrm{s}$.

$\rho_{b} \quad$ soil dry bulk density, $\mathrm{kg} / \mathrm{m}^{3}$.

Pa ambient gas phase density, $\mathrm{kg} / \mathrm{m}^{3}$.

$\tau \quad$ gas travel time to well screen. $s$.

$i_{R} \quad$ retarded gas travel time to well screen, $s$.

$\Psi_{d} \quad$ normalized streamfunction.

$\xi \quad$ dimensionless source surength defined by equation (15). 


\section{REFERENCES}

1. Wilson, D.E., R.E. Montgomery, and M.R. Sheller, A Mathematical model for removing volatile subsurface hydrocarbons by miscible displacement, Water, Air, Soil Pollut., 33, 231-255, 1987.

2. Metcaif, D.E. and G.J. Farquhar, Modeling gas migration through unsaturated soils from waste disposal sites, Water, Air, Soil Pollut., 32, 247-257, 1987.

3. Pruess, K., TOUGH user's guide, Nucl. Regul. Comm. Rep. NUREG/CR-4645, Washington, D.C., June, 1987.

4. Baehr, A.L., G.E. Hoag, and M.C. Marley, Removing volatile contaminants from the unsaturated zone by inducing advective air-phase transport, J. Contam. Hydrol., 4, 1-26, 1989.

5. Falta. R.W., I. Javandel, K. Pruess, a.d P.A. Witherspoon, Density-driven flow of gas in the unsaturated zone due to the evaporation of volatile organic compounds, Water Resour. Res., 25(10), 2159-2169, 1989.

6. Sieep, B.E. and J.F. Sykes. Modeling the transport of volatile organics in variably saturated media, Water Resour. Res., 25(1), 81-92, 1989.

7. Falta. R.W., K. Pruess, I. Javandel. and P.A. Witherspoon, Numerical modeling of steam injection for the removal of nonaqueous phase liquids from the subsurface, 1. Numerical formulation. Water Resour. Res., 28(2), 433-449, 1992.

8. Mendoza. C.A. and E.O. Frind. Advective-dispersive transport of dense organic vapors in the unsaturated zone. 1. Model development, Water Resour. Res., 26(3), 379-387, 1990. 
9. Johnson, P.C, M.W. Kemblowski, and J.D. Colthart, Practical screening models for soil venting applications, Proceedings of Petroleum Hydrocarbons and Organic Chemicals in Ground Water, Natl. Water Well Assoc., Houston, TX, 521-546, 1988.

10. Massmann, J.W., Applying groundwater models in vapor extraction system design, J. Environ. Eng., 115(1), 129-149, 1989.

11. Johnson, P.C., M.W. Kemblowski, and J.D. Colthart, Quantitative analysis for the cleanup of hydrocarbon-contaminated soils by in-situ soil venting, Ground Water. 28(3), 413-429, 1990.

12. Johnson, P.C., C.C. Staniey, M.W. Kemblowski, D.L. Byers, and J.D. Colthart, A practical approach to the design, operation, and monitoring of in situ soil-venting systems, Ground Water Monit Rev., 10(2), 159-178, 1990.

13. McWhorter, D.B., Unsteady radial How of gas in the vadose zone, J. Contam. Hydrol., 5, 297-314, 1990.

14. Wilson, D.J., A.N. Clarke, and J.H. Clarke, Soil clean up by in-situ aeration, 1 , Mathematical modeling, Sep. Sci. and Tech., 23(10), 991-1037, 1988.

15. Baehr, A.L. and M.F. Hult. Evaluation of unsaturated zone air permeability through pneumatic tests, Water Resour. Res., 27(10), 2605-2617, 1991.

16. Shan, C., R.W. Falta, and I. Javandel. Analytical solutions for steady state gas flow to a soil vapor extraction well, Water Resour. Res., 28(4), 1105-1120, 1992.

17. Ho, C.K. and K.S. Udell. A mass transier model for the removal of a volatile organic compound from heterogeneous porous media during vacuum extraction, 
Heat Transfer in Geophysical Media, ASME, HTD-Vol. 172, 55-62, 1991.

18. Javandel, I., C. Doughty, and C.F. Tsang, Groundwater Transport: Handbook of Mathematical Models, American Geophysical Union, Washington, D.C., 1984.

19. Jury, W.A., W.F. Spencer, and W.J. Farmer, Behavior assessment model for trace organics in soil, 1, Model description, J. Environ. Qual., 12(4), 558-564, 1983.

20. Karickhoff, S.W., D.S. Brown, and T.A. Scott, Sorption of hydrophobic pollutants on natural sediments, Water Res., 13, 241-248, 1979.

21. Schwartzenbach, R.P. and J. Westfall, Transport of non-polar organic compounds from surface water to groundwater, Laboratory sorption studies, Environ. Sci. Technol., 15, 1360-1375, 1981.

22. Lyman, W.J., W.F. Reehl, and D.H. Rosenblath, Handbook of Chemical Propenty Estimation Methods: Environmental Behavior of Organic Compounds, McGrawHill, New York. 1982.

23. Mendoza. C.A. and E.O. Frind, Advective-dispersive transport of dense organic vapors in the unsaturated zone, 2. Sensitivity analysis, Water Resour. Res., 26(3), 388-398, 1990.

24. Reid, R.C.. J.M. Prausnitz, and B.E. Poling, The Properties of Gases and Liquids, McGraw-Hill. New York. 1987.

25. Mercer, J.W. and R.M. Cohen, A review of immiscible fluids in the subsurface: Properties, models, characterization and remediation, J. Contam. Hydrol., 6, 107. 163. 1990. 


\section{Table 1. Radial Flow Problem Specifications}

\section{Parameter}

Value

\begin{tabular}{|ll|}
\hline Thickness & $\mathrm{h}=10 \mathrm{~m}$ \\
Effective gas permeability & $\mathrm{k}=1 \times 10^{-12} \mathrm{~m}^{2}$ \\
Porosity & $\varphi=0.4$ \\
Residual water saturation & $\mathrm{S}_{\mathrm{w}}=0.025$ \\
Well radius & $\mathrm{r}_{\mathrm{w}}=0.051 \mathrm{~m}$ \\
Radius of influence & $\mathrm{r}_{\mathrm{r}}=30.0 \mathrm{~m}$ \\
Well pressure & $\mathrm{P}_{\mathrm{w}}=0.7 \mathrm{ATM}=70,927 \mathrm{~Pa}$ \\
Temperature & $\mathrm{T}=20^{\circ} \mathrm{C}$ \\
Gas viscosity & $\mu=1.81 \times 10^{-5} \mathrm{~kg} / \mathrm{m} \cdot \mathrm{s}$ \\
Soil bulk density & $\mathrm{P}_{\mathrm{b}}=1500 \mathrm{~kg} / \mathrm{m}^{3}$ \\
Contaminant & $\mathrm{Trichloroethylene}$ \\
Henry's Constant & $\mathrm{H}=0.353$ \\
Soil-wa:er partition coefficient & $\mathrm{K}_{\mathrm{D}}=0.000126 \mathrm{~m} 3 / \mathrm{kg}$ \\
Gas phase retardation coefficient & $\mathrm{R}_{\mathrm{g}}=3.73$ \\
\hline
\end{tabular}




\section{Table 2. Two-Dimensional Flow Problem Specifications for Cylindrical and Conical Initial Contaminant Distributions}

\section{Parameter}

\section{Value}

\begin{tabular}{|ll|}
\hline Depth to impermeable boundary & $\mathrm{h}=40 \mathrm{~m}$ \\
Depth to bottom of screen & $\mathrm{a}=30 \mathrm{~m}$ \\
Depth to top of screen & $\mathrm{b}=20 \mathrm{~m}$ \\
Total gas removal rate & $\mathrm{Q}_{\mathrm{a}}=0.155 \mathrm{~m} / \mathrm{s}$ \\
Effective horizontal gas permeability & $\mathrm{k}_{\mathrm{h}}=2 \times 10^{-11} \mathrm{~m}^{2}$ \\
Effective vertical gas permeability & $\mathrm{k}_{\mathrm{v}}=2 \times 10^{-11} \mathrm{~m}^{2}$ \\
Porosity & $\varphi=0.4$ \\
Residual water saturation & $\mathrm{S}_{\mathrm{w}}=0.25$ \\
Temperature & $\mathrm{T}=10^{\circ} \mathrm{C}$ \\
Gas viscosity & $\mu=1.76 \times 10^{-5} \mathrm{~kg} / \mathrm{m} \cdot \mathrm{s}$ \\
Gas phase retardation coefficient & $\mathrm{R}_{\mathrm{g}}=1.0$ \\
\hline
\end{tabular}


Table 3. Two-Dimensional Flow Problem Specifications for Uniform Initial Contaminant Distribution

\begin{tabular}{|ll|}
\multicolumn{1}{c|}{ Parameter } & \multicolumn{1}{c|}{ Value } \\
\hline Depth to impermeable boundary & $\mathrm{h}=10 \mathrm{~m}$ \\
Depth to bottom of screen & $\mathrm{a}=7 \mathrm{~m}$ \\
Depth to top of screen & $\mathrm{b}=3 \mathrm{~m}$ \\
Total gas removal rate & $\mathrm{Q}_{\mathrm{a}}=0.081 \mathrm{~m}^{3} / \mathrm{s}$ \\
Effective horizontal gas permeability & $\mathrm{k}_{\mathrm{h}}=1 \times 10^{-11} \mathrm{~m}^{2}$ \\
Effective vertical gas permeability & $\mathrm{k}_{\mathrm{v}}=1 \times 10^{-12} \mathrm{~m}^{2}$ \\
Porosity & $\varphi=0.4$ \\
Residual water saturation & $\mathrm{S}_{\mathrm{w}}=0.0$ \\
Temperature & $\mathrm{T}=10^{0} \mathrm{C}$ \\
Gas viscosity & $\mu=1.76 \times 10^{-5} \mathrm{~kg} / \mathrm{m} \cdot \mathrm{s}$ \\
Gas phase retardation coefficient & $\mathrm{R}_{\mathrm{g}}=1.0$ \\
Initial contaminant gas mole fraction & $\chi_{0}=0.01$ \\
\hline
\end{tabular}


Table 4. Two-Dimensional Flow Problem Specifications for Layered Initial Contaminant Distribution

Parameter

Value

\begin{tabular}{|c|c|}
\hline Depth to impermeable boundary & $\mathrm{h}=10 \mathrm{~m}$ \\
\hline Depth to bottom of screen & $\mathrm{a}=10 \mathrm{~m}$ \\
\hline Depth to top of screen & $\mathrm{b}=7 \mathrm{~m}$ \\
\hline Total gas removal rate & $\mathrm{Q}_{\mathrm{a}}=0.0605 \mathrm{~m} 3 / \mathrm{s}$ \\
\hline Effective horizontal gas permeability & $\mathrm{k}_{\mathrm{h}}=1 \times 10^{-11} \mathrm{~m}^{2}$ \\
\hline Effective vertical gas permeability & $\mathrm{k}_{\mathrm{v}}=1 \times 10^{-12} \mathrm{~m}^{2}$ \\
\hline Porosity & $\varphi=0.4$ \\
\hline Residual water saturation & $S_{w}=0.25$ \\
\hline Temperature & $\mathrm{T}=10^{\circ} \mathrm{C}$ \\
\hline Gas viscosity & $\mu=1.76 \times 10^{-5} \mathrm{~kg} / \mathrm{m} \cdot \mathrm{s}$ \\
\hline Soil bulk density & $\rho_{b}=1590 \mathrm{~kg} / \mathrm{m}^{3}$ \\
\hline Contaminant & Trichloroethylene \\
\hline Henry's Constant & $\mathrm{H}=0.214$ \\
\hline Soil-water partition coefficient & $\mathrm{K}_{\mathrm{D}}=0.000126 \mathrm{~m}^{3} / \mathrm{kg}$ \\
\hline Gas phase retardation coefficient & $\mathrm{R}_{\mathrm{g}}=5.68$ \\
\hline \multicolumn{2}{|l|}{ Initial contaminant gas mole fraction: } \\
\hline $0 \leq z \leq 3 m$ & $\chi_{0}=0.0$ \\
\hline $3 m \leq z \leq 4 m$ & $\chi_{0}=0.002$ \\
\hline $4 m \leq z \leq 5 m$ & $\chi_{0}=0.004$ \\
\hline $5 m \leq z \leq 6 m$ & $\chi_{0}=0.006$ \\
\hline $6 m \leq z \leq 7 m$ & $\chi_{0}=0.010$ \\
\hline $7 m \leq z \leq 10 m$ & $\chi_{0}=0.0$ \\
\hline
\end{tabular}




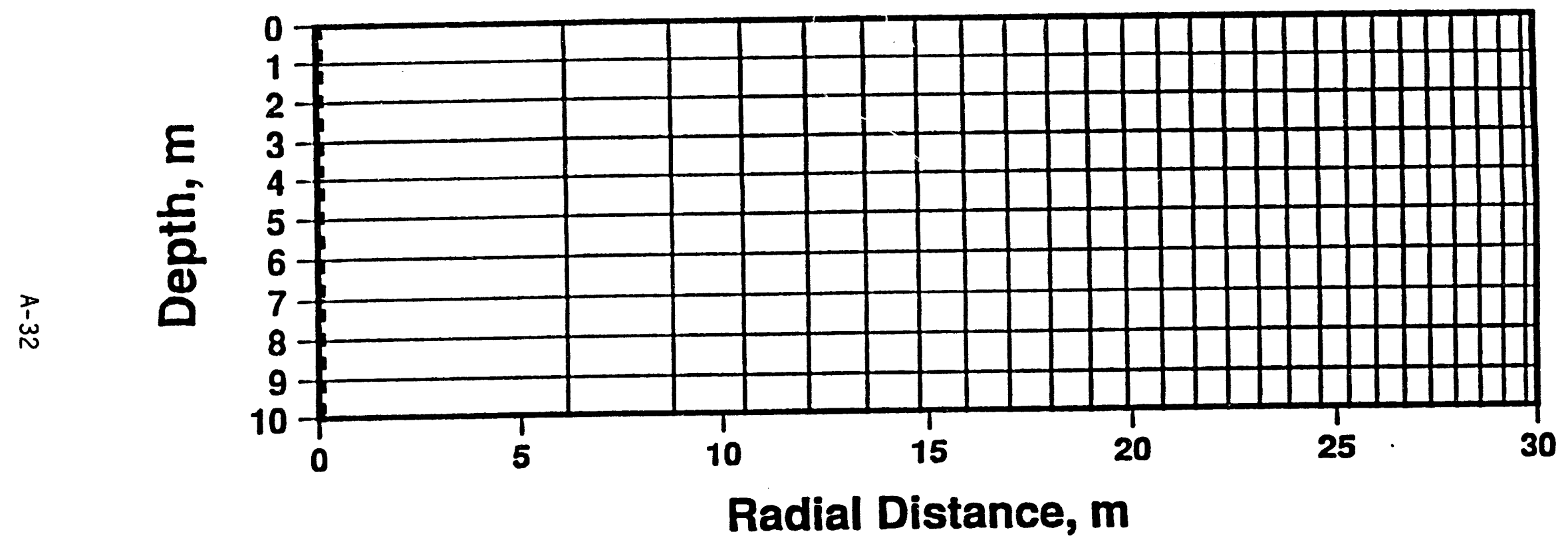

Figure 1. Normalized streamfunction and retarded travel time distributions for radial flow example. 


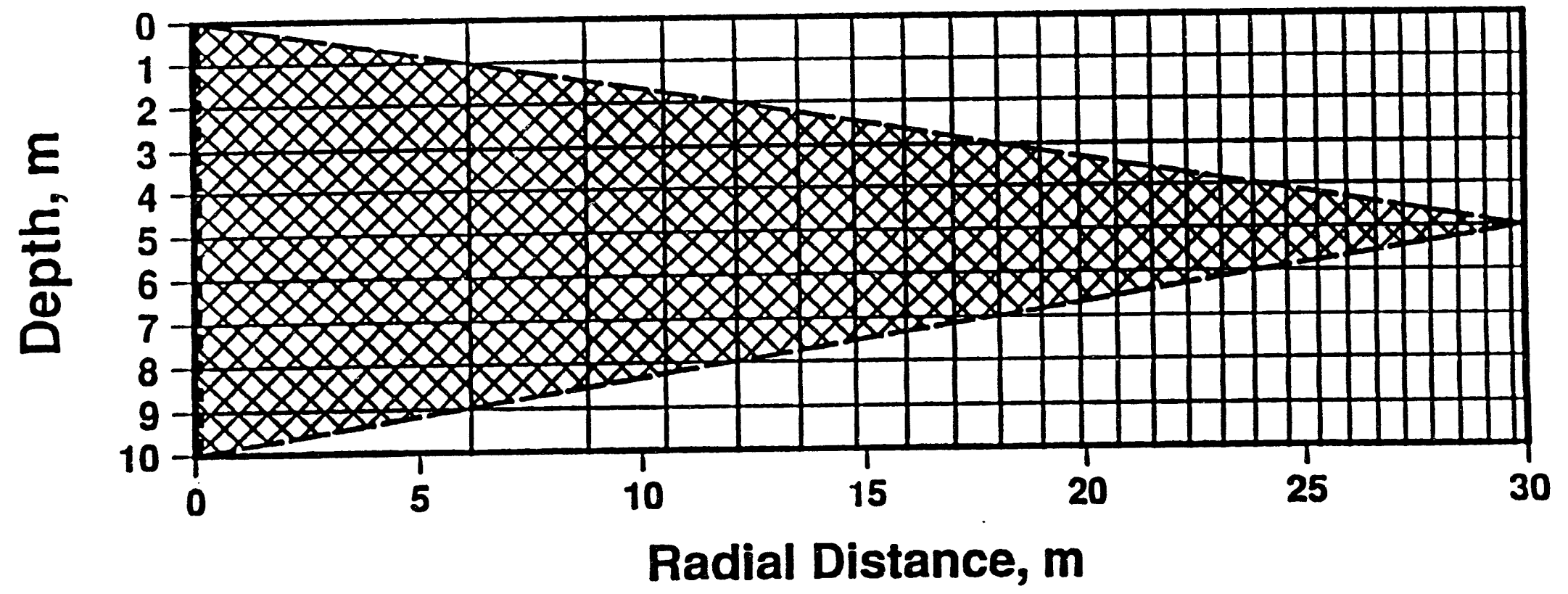

Figure 2. Initial TCE contaminated zone geometry. 


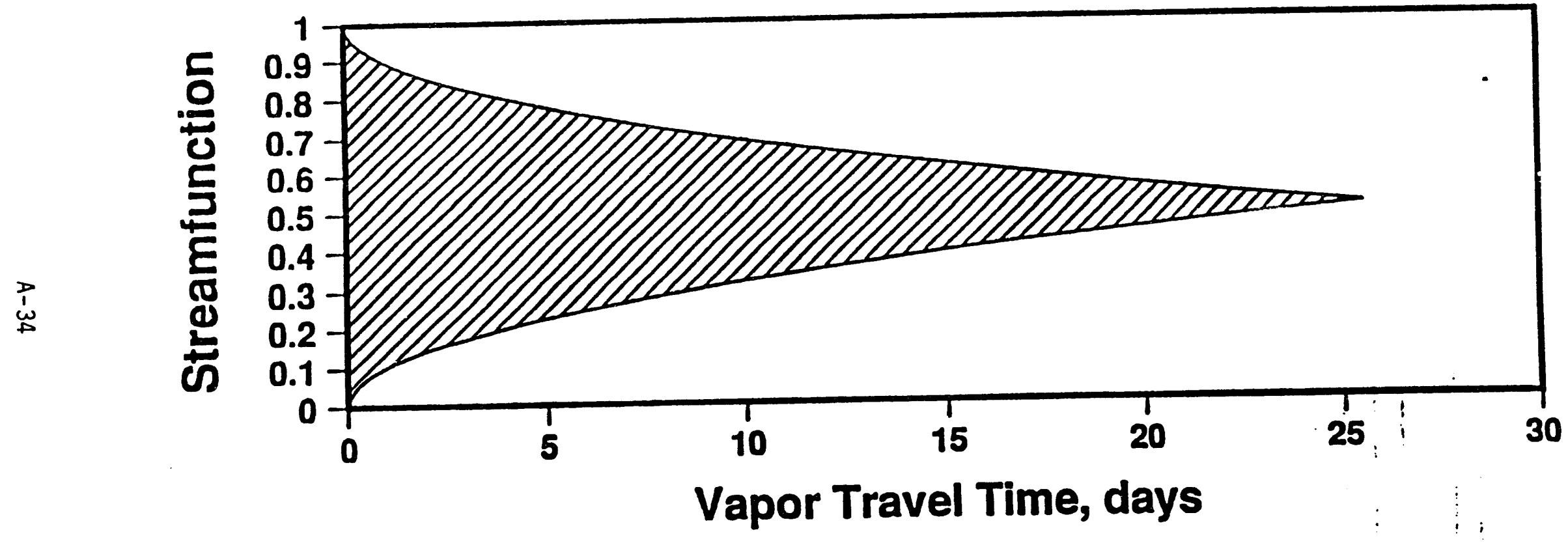

Figure 3. Transformed TCE contaminated zone. 


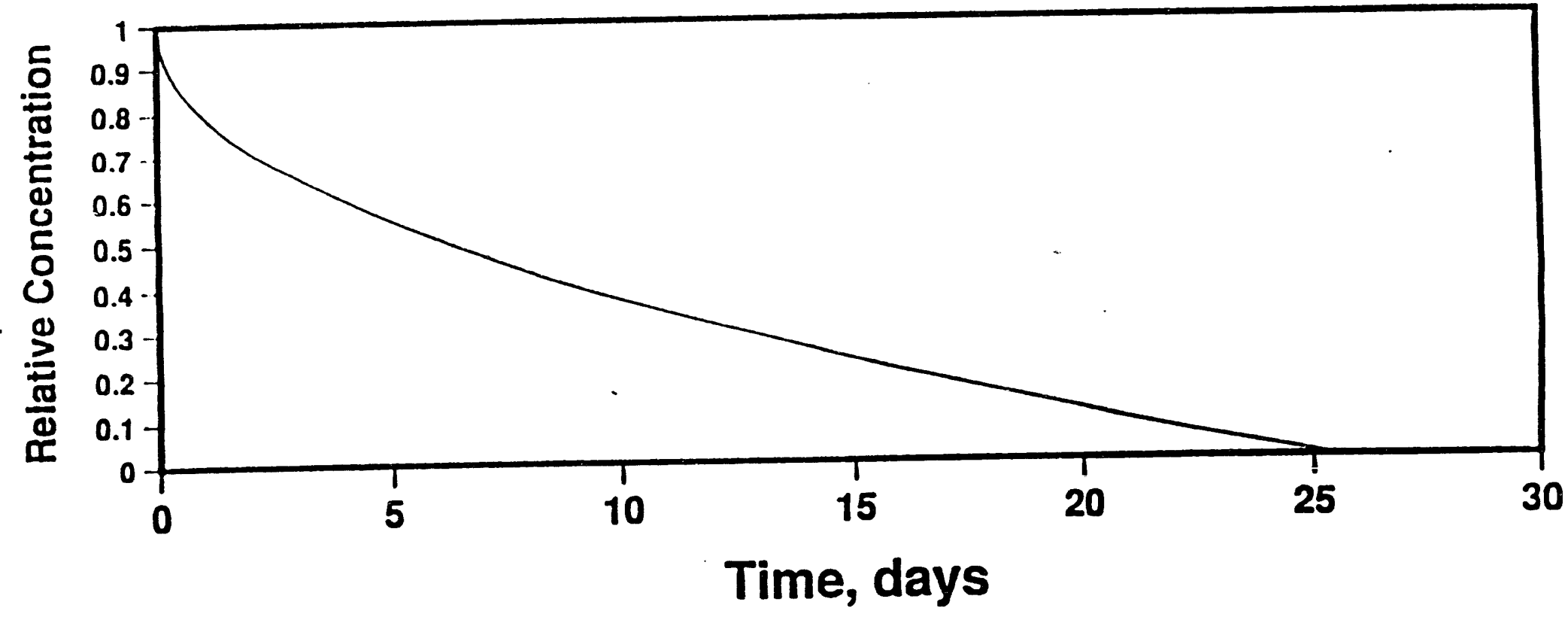

Figure 4. Relative mole fraction versus retarded travel time. 


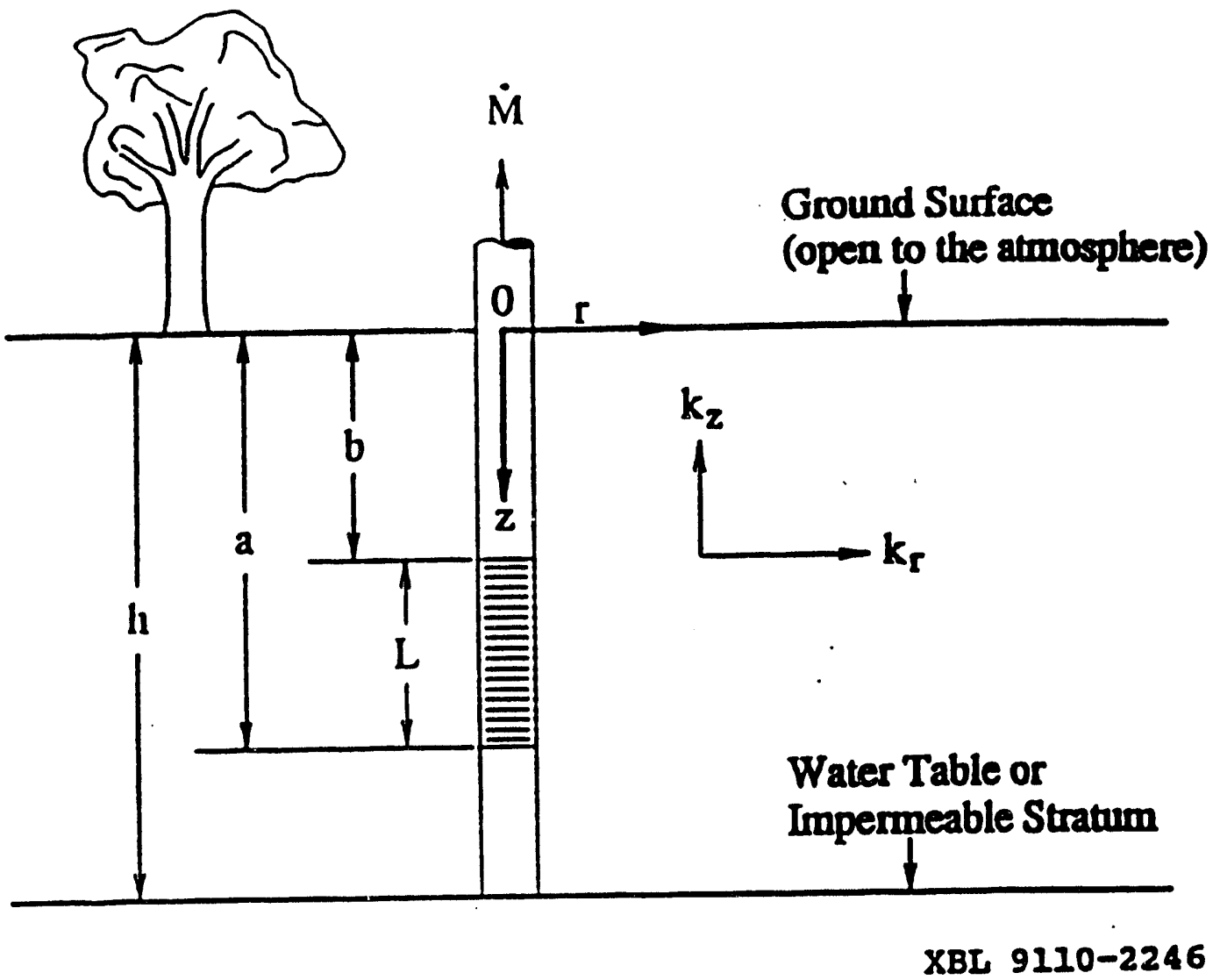

Figure 5. Well geonetry for two-dimensional flow examples. 


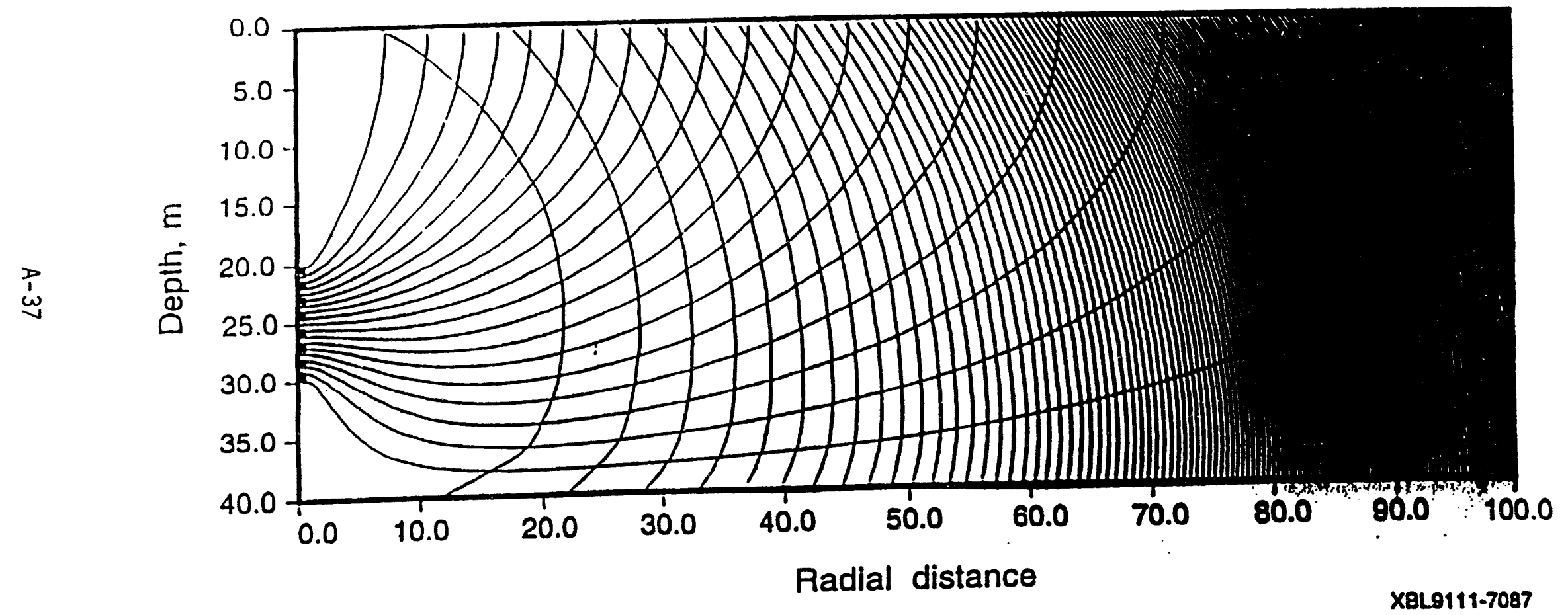

Figure 6. Normalized streamfunction and gas travel time distributions for twodimensional flow. 


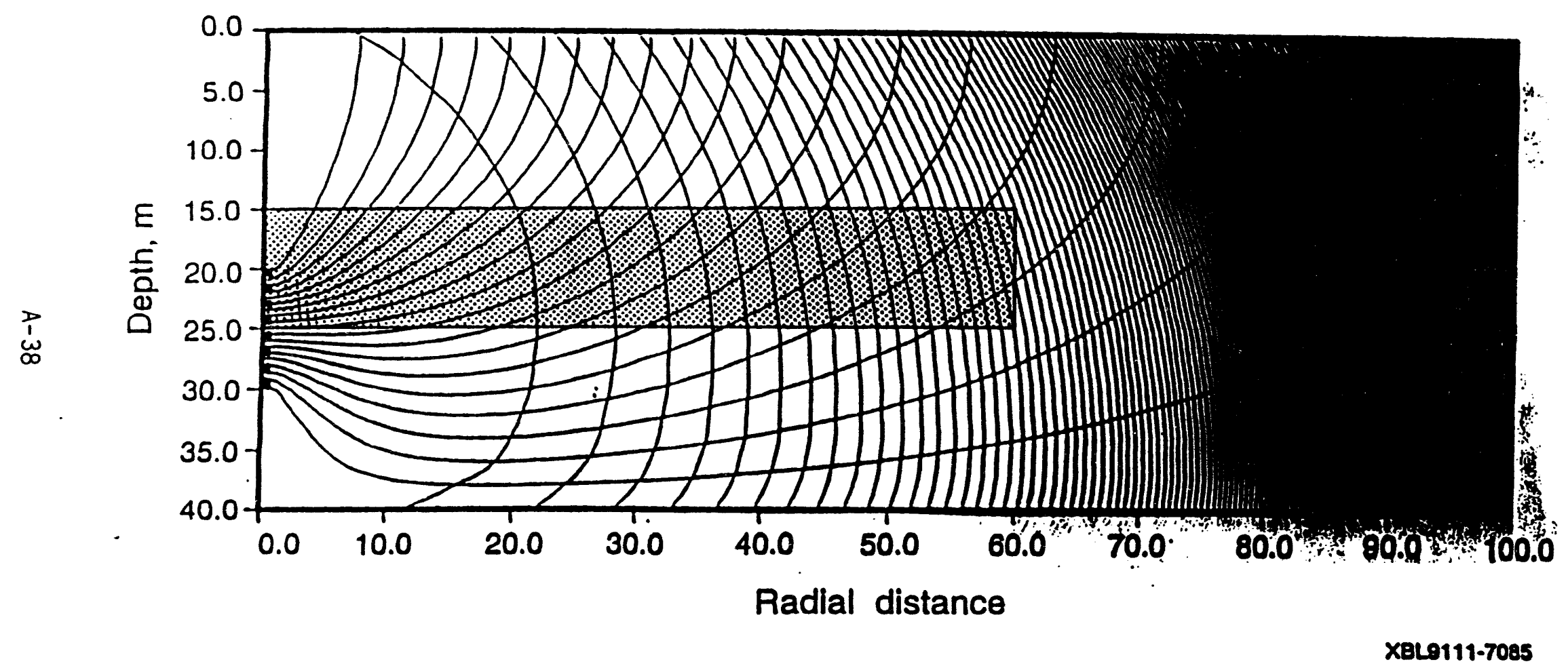

Figure 7. Initial VOC contaminated zone geometry. 


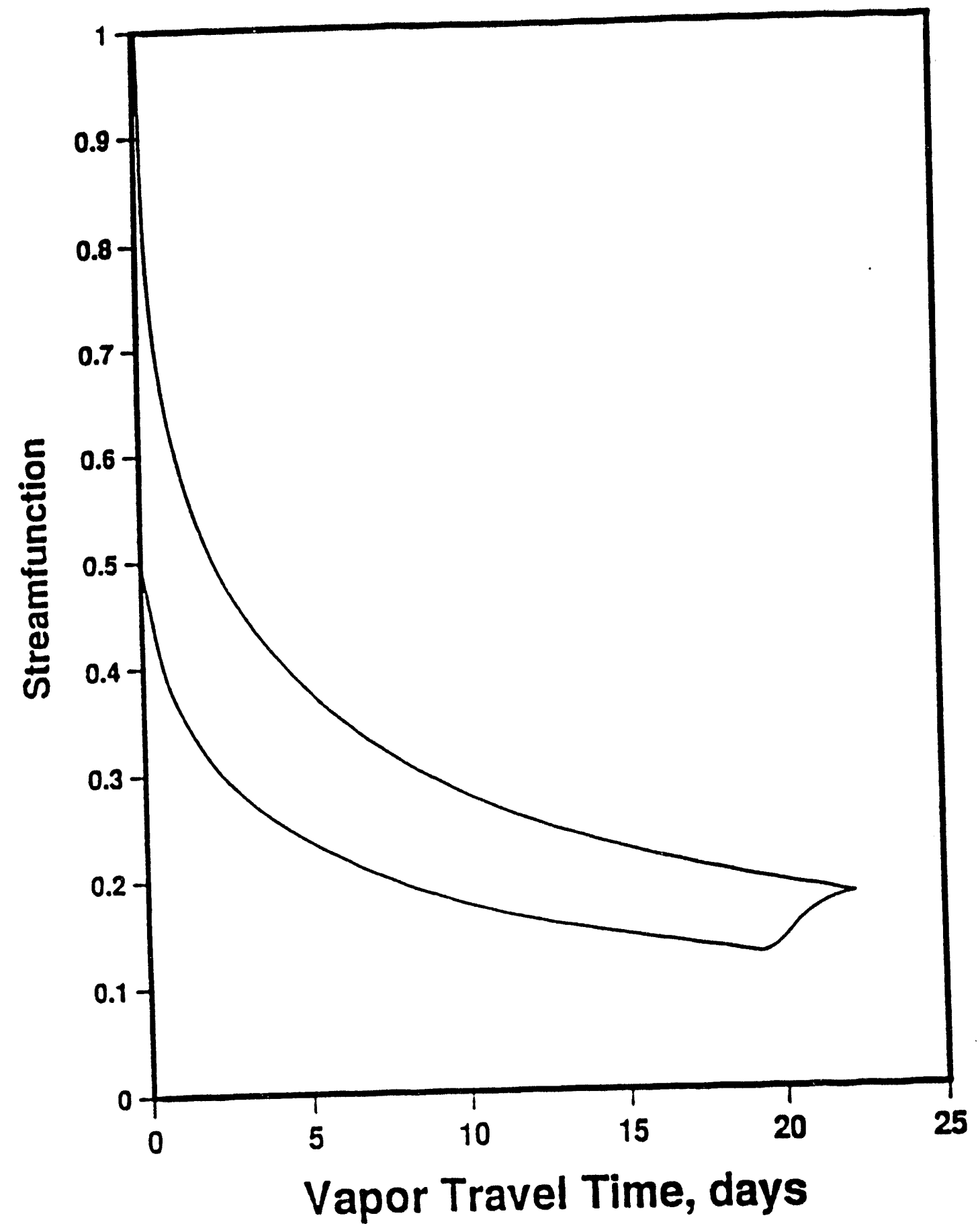

Figure 8. Transformed VOC contaminated zone. 


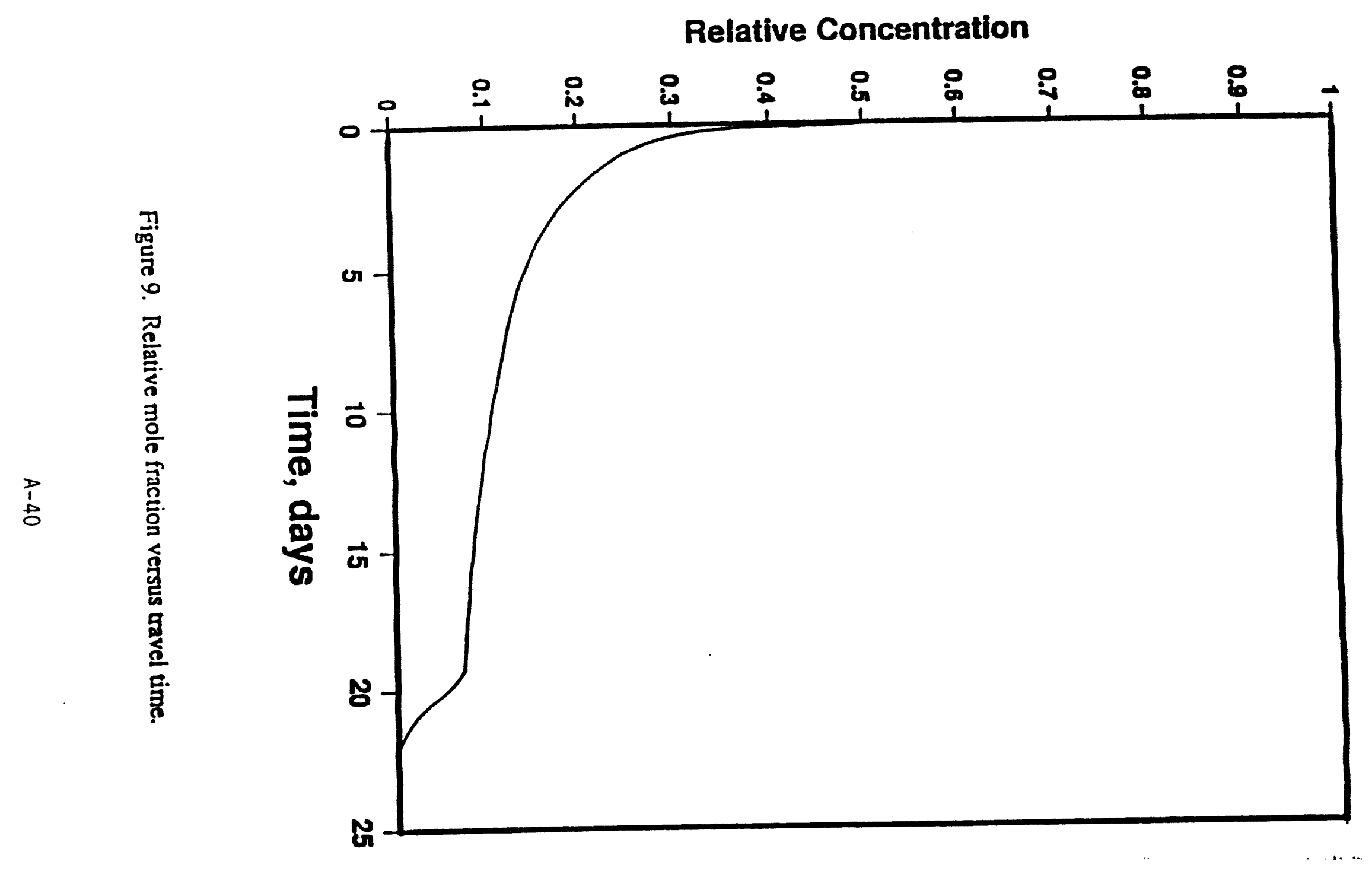




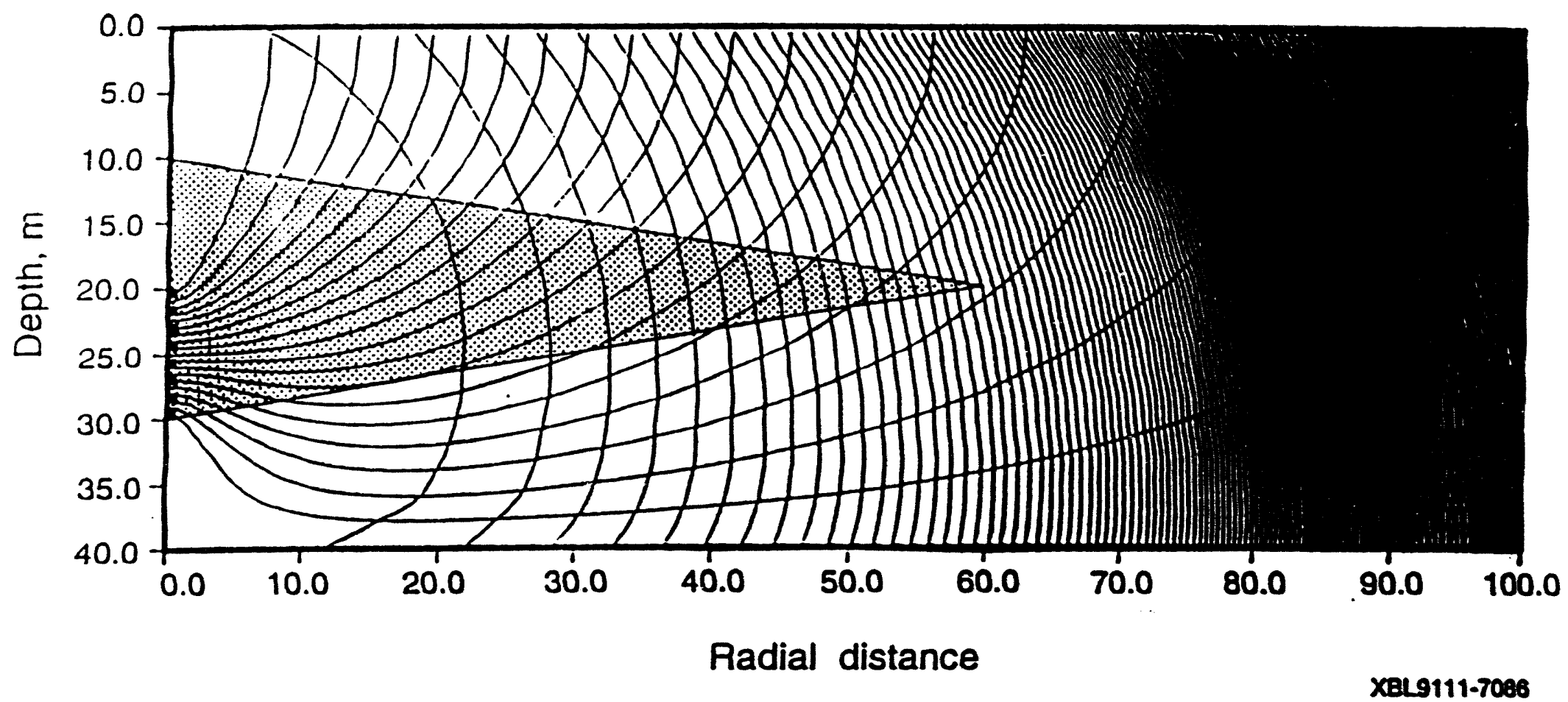

Figure 10. Initial VOC contaminated zone geometry. 


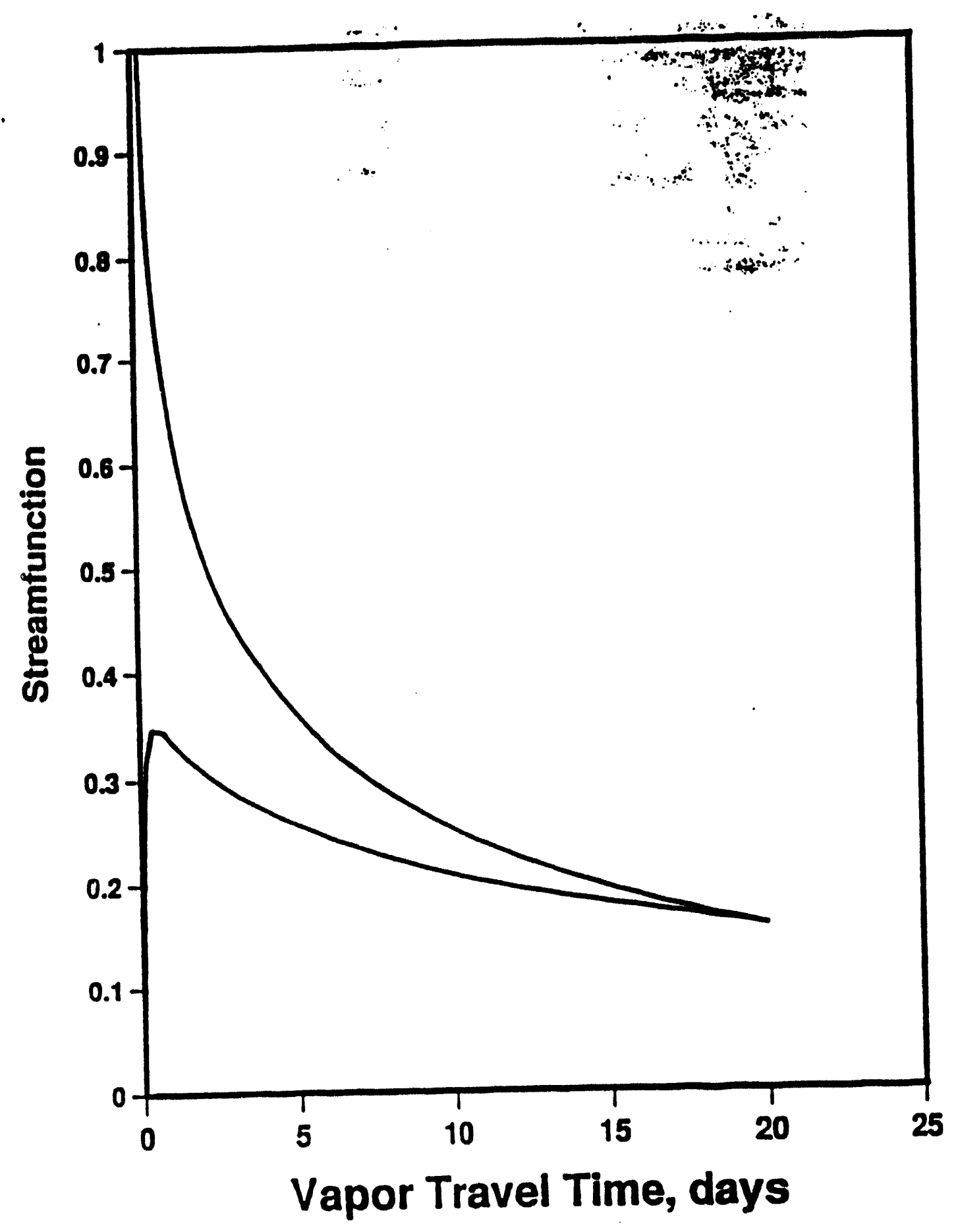

Figure 11. Transformed VOC contaminated zone. 


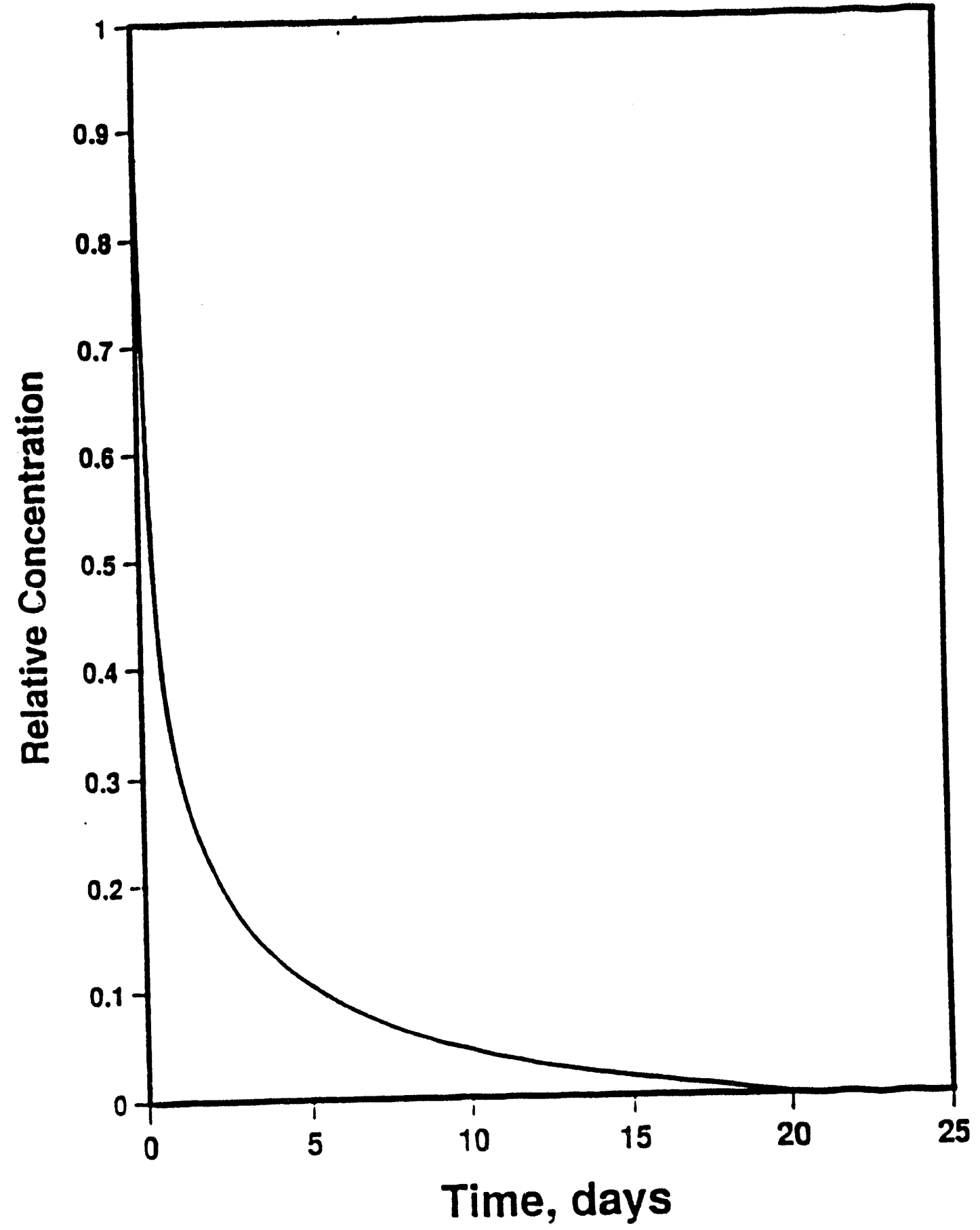

Figure 12. Relative mole fraction versus travel time. 


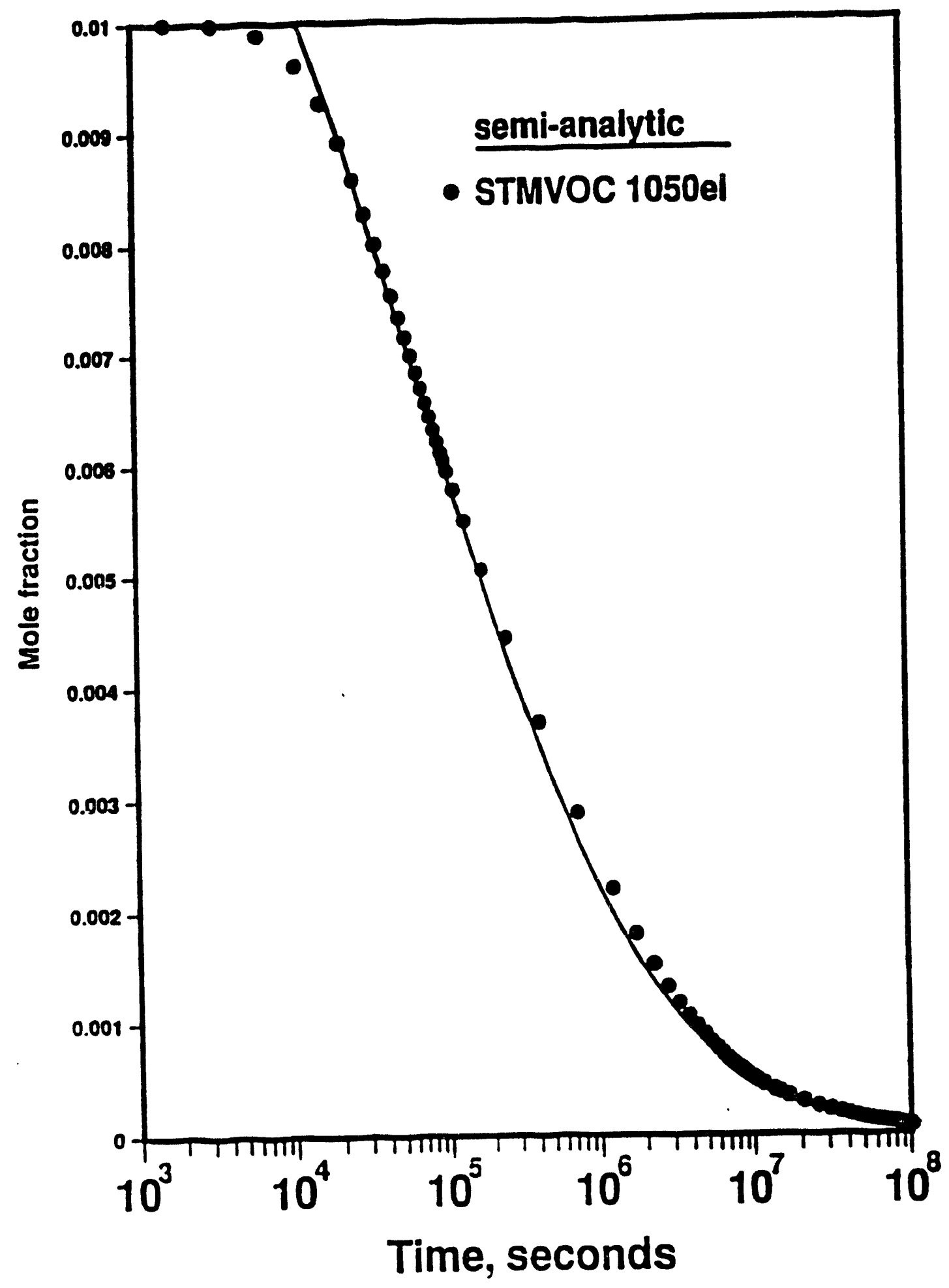

Figure 13. Comparison of numerical results with analytical solution for uniform initial VOC distribution. 


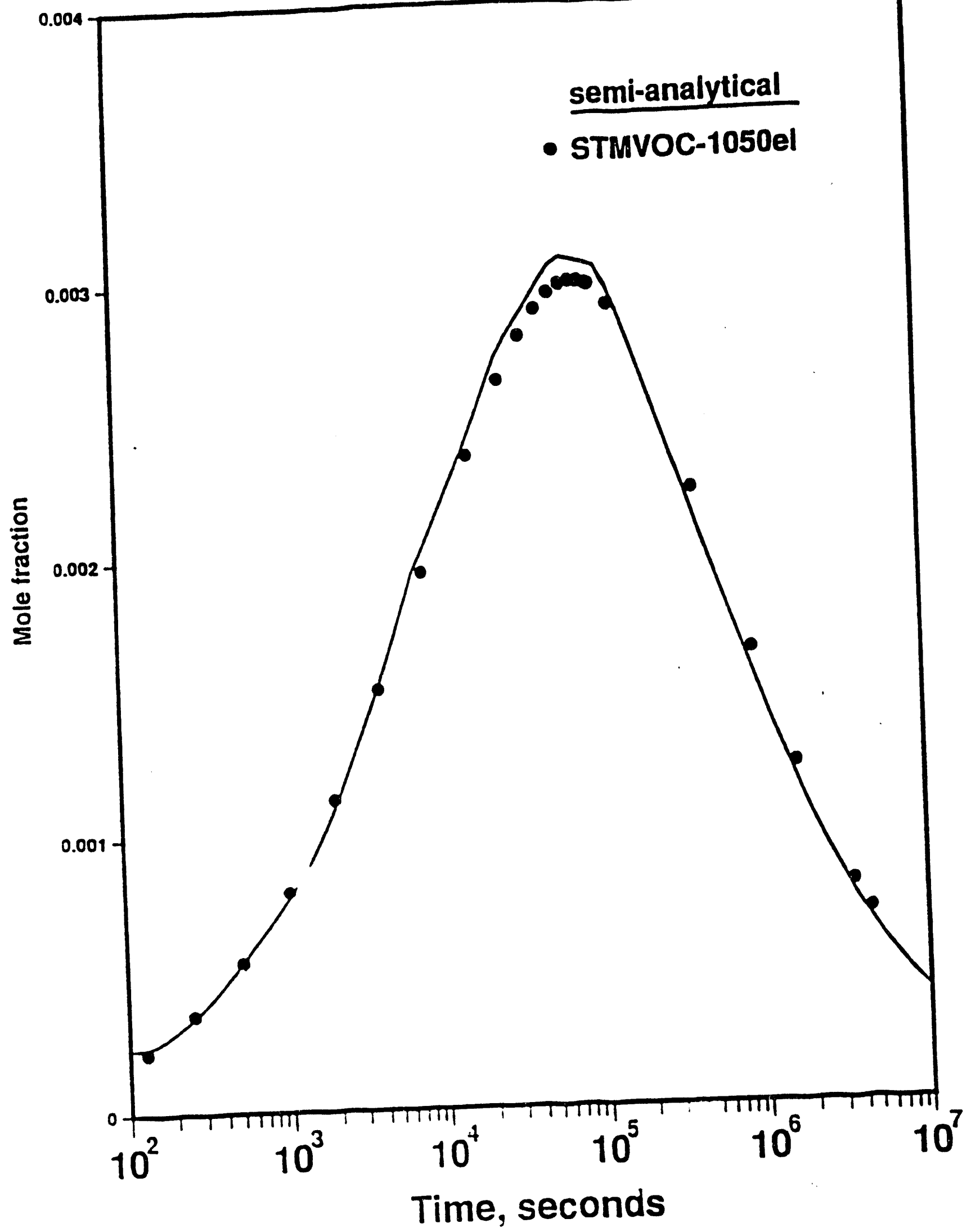

Figure 14. Comparison of numerical results with analytical solution for layered initial TCE distribution. 


\title{
ANalytical SOlutions for Gas Flow DUE to Gas INJECTION AND EXTRACTION FROM HORIZONTAL WELLS
}

Ronald W. Falta

Department of Earth Sciences, Clemson University, Clemson, SC

August 15, 1992

\begin{abstract}
Analytical solutions are developed for modeling the transient and steady state gas pressure and the steady state streamfunction fields resulting from gas injection and extraction from a pair of parallel horizontal wells. These solutions apply in cases in which the ground surface is open to the atmosphere, and in which the porous media is isotropic but homogeneous. By neglecting end effects due to the finite length of the wells, the threedimensional gas flow field is approximated as a two-dimensional cross-section perpendicular to the wells. These solutions may be used to develop estimates of the horizontal well system behavior, and are useful for model verification.
\end{abstract}




\section{INTRODUCTION}

Soil vapor extraction is a new method for removing volatile organic contaminants from the unsaturated zone. A soil vapor extraction system consists of one or more pumping wells screened in the unsaturated zone. These wells may have either a vertical or a horizontal configuration, and additional wells may be used for gas injection. Contaminant removal is achieved as the induced gas flow sweeps the contaminated zone, flushing contaminant vapors from the soil. Although the majority of soil vapor extraction operations have involved the use of vertical wells (see, for example, [Johnson et al., 1990b]), researchers have recently begun to evaluate the performance of horizontal well vapor extraction systems [Looney et al., 1991].

In the design and evaluation of vapor extraction systems, mathematical models of the gas flow field are essential tools. Usually, these models are based on numerical solutions of the governing partial differential equations, but for certain well geometries and conditions, it is possible to develop analytical solutions for modeling the gas flow. While analytical solutions have been developed for gas flow to a vertical well [Johnson et al., 1988; Massmann, 1989; Johnson et al., 1990a,b; McWhorter, 1990; Baehr and Hult, 1991; Shan et al., 1992], and to a point sink [Wilson et al., 1988], the author is not aware of any analytical solutions for gas flow to a system of horizontal wells.

In this paper, analytical solutions are developed for both transient and steady state gas flow to a pair of horizontal wells in the unsaturated zone. The steady state solutions include both the pressure distribution and the streamfunction distribution. A schematic diagram of the well geometry considered in these solutions is shown in Figure 1. In general, the two wells shown in this figure may be both extracting gas, both 
injecting gas, or one may be injecting gas while the other is producing gas. In field applications, it is likely that gas would be injected in the lower well and produced from the upper well. It is assumed that the system is bounded below by a no-flow boundary (either the water table or an impermeable stratum), and the upper surface is assumed to be open to the atmosphere (constant pressure). The two wells are assumed to be parallel, and the porous media is homogerieous, but may be anisotropic. The well system is modeled as a two-dimensional cross-section through the wells (the line marked A-A in Figure 1), and end effects due to the finite lengths of the wells are not considered.

\section{MATHEMATICAL MODEL}

For transient gas flow in an anisotropic porous medium neglecting gravity, the governing equation is given by [Bear, 1972]

$$
\frac{\phi \mu}{P} \frac{\partial P^{2}}{\partial t}=\nabla \cdot\left(\tilde{k} \nabla P^{2}\right)
$$

where $\phi$ is the volumetric gas content, $\mu$ is the dynamic gas viscosity, $P$ is the gas pressure, and $\bar{k}$ is the effective gas phase permeability tensor. In (1), the system is assumed to be isothermal, the gas is assumed to behave as an ideal gas with a constant viscosity and composition, and the movement of pore water is neglected. It is also assumed that Darcy's law is valid for the gas flow and that Klinkenberg effects [Klinkenberg, 1941] are negligible. This nonlinear equation may be approximated by a linear equation by replacing $P$ in the denominator of the left hand side of (1) by the ambient pressure, $P_{a}$,

$$
\frac{\phi \mu}{P_{a}} \frac{\partial P^{2}}{\partial t}=\nabla \cdot\left(\bar{k} \nabla P^{2}\right)
$$


For problems in which the maximum pressure drop is less than one half of the ambient pressure, the error resulting from this linearization is small [Kidder, 1957]. Analyses of the importance of the various assumptions used to derive (2) are given by Massmann [1989], McWhorter [1990], and Baehr and Hult [1991].

Referring to Figure 2, we wish to solve (2) in a horizontally infinite system subject to gas injection or removal in the two wells at mass rates of $\dot{M}_{1}$ and $\dot{M}_{2}$ per unit length of well screen. In the analytical solutions, the screened portion of the wells are approximated as line sinks (or sources) of uniform strength. While this approximation will lead to some errors in the pressure distribution in the near well screen [Bear, 1979], these errors are not expected to be large, and the use of uniform strength line sinks to approximate well screens is common in hydrogeology.

The boundary conditions for this problem consist of constant (atmospheric) pressure at the ground surface, and a no flow boundary at a depth of $h$,

$$
P=P_{a} \text { at } z=0
$$

and

$$
\frac{\partial P}{\partial z}=0 \quad \text { at } \quad z=h
$$

\section{Transient Pressure Solution}

To simplify the solution process, it is convenient to introduce a new variable, $u$, (see, for example, Collins [19611) defined as

$$
u=P_{a}^{2}-P^{2}
$$


Substituting (5) into (2) results in

$$
\frac{\phi \mu}{P_{a}} \frac{\partial u}{\partial t}=\nabla \cdot(\tilde{k} \nabla u)
$$

and (3) and (4) become

$$
u=0 \text { at } z=0
$$

and

$$
\frac{\partial u}{\partial z}=0 \text { at } z=h
$$

We will first consider the pressure solution due to gas injection or extraction in one of the wells, with a gas mass rate of $\dot{M}$. The solution for the two-well problem is then obtained by superposition. A solution to (6) for an instantaneous point source of strength $Q$ located at $x=x^{\prime}, y=y^{\prime}, z=z^{\prime}$ in an infinite, anisotropic porous media may be adapted from the heat conduction solution given by Carslaw and Jaeger [1959] to get

$$
u=\frac{Q(\phi \mu)^{3 / 2}}{8\left(\pi^{3} t^{3} P_{a}^{3} k_{x} k_{y} k_{z}\right)^{1 / 2}} \operatorname{cxp}\left\{-\frac{\phi \mu}{4 P_{a} t}\left[\frac{\left(x-x^{\prime}\right)^{2}}{k_{x}}+\frac{\left(y-y^{\prime}\right)^{2}}{k_{y}}+\frac{\left(z-z^{\prime}\right)^{2}}{k_{z}}\right]\right\}
$$

where $k_{x}, k_{y}$, and $k_{z}$ are the effective gas phase permeabilities in the $x, y$, and $z$ directions, respectively. To develop the solution for an instantaneous line source located at $x=x^{\prime}, z=z^{\prime},(9)$ is integrated with respect to $y^{\prime}$ :

$$
u=\frac{Q \phi \mu}{4 \pi i P_{a} \sqrt{k_{x} k_{z}}} \exp \left\{-\frac{\phi \mu}{4 P_{a} l}\left[\frac{\left(x-x^{\prime}\right)^{2}}{k_{x}}+\frac{\left(z-z^{\prime}\right)^{2}}{k_{z}}\right]\right\}
$$

To obtain the solution for a continuous line source/sink per unit screen length of strength $q,(10)$ is integrated with respect to time:

$$
u=\frac{q \phi \mu}{4 \pi P_{a} \sqrt{k_{x} k_{2}}} \int_{0}^{1} \frac{\exp \left\{\frac{-r^{2} \phi \mu}{4 P_{a}\left(t-t^{\prime}\right)}\right\}}{t-t^{\prime}} d t^{\prime}
$$


or

$$
u=\frac{q \phi \mu}{4 \pi P_{a} \sqrt{k_{x} k_{z}}} \int_{\frac{r_{\Delta \mu}}{4 p_{a}}}^{-} \frac{e^{-v}}{v} d v
$$

where

$$
q=\frac{2 P_{a} R T \dot{M}}{\phi M_{m}}
$$

and

$$
r^{2}=\frac{\left(x-x^{\prime}\right)^{2}}{k_{z}}+\frac{\left(z-z^{\prime}\right)^{2}}{k_{z}}
$$

In (13), $R$ is the universal gas constant, $T$ is the absolute temperature, $\dot{M}$ is the gas mass injection/production rate per unit screen length, and $M_{w}$ is the average molecular weight of the soil gas. For gas production, $\dot{M}$, and hence $q$ is positive, while for gas injection, $\dot{M}$ and $q$ are negative. Defining:

$$
\begin{gathered}
\alpha_{x 2}=\frac{\sqrt{k_{x} k_{2} P_{a}}}{\phi \mu} \\
\alpha_{2}=\frac{k_{2} P_{a}}{\phi \mu}
\end{gathered}
$$

and

$$
\hat{i}=x \sqrt{\frac{k_{z}}{k_{x}}}
$$

and considering a line source located at $x^{\prime}=0, z=z^{\prime},(12)$ may be written as

$$
u=\frac{q}{4 \pi \alpha_{x z}} E_{1}\left\{\frac{\hat{x}^{2}+\left(z-z^{\prime}\right)^{2}}{4 \alpha_{2} t}\right\}
$$


in which $E_{1}(v)$ is the exponential integral.

The boundary conditions, (7) and (8) are applied by using the method of images with the following sources and sinks (see Shan et al. [1992]; Wilson et al. [1988])

$$
\text { sinks at: } z_{n}^{+}=(-1)^{n} z^{\prime} \pm 2 n h, n=0,1,2,3, \cdots
$$

and

$$
\text { sources at: } z_{n}^{-}=(-1)^{n+1} z^{\prime} \pm 2 n h, n=0,1,2,3, \cdots
$$

Then the transient pressure solution for a single horizontal well neglecting end effects is

$$
u=\frac{q}{4 \pi \alpha_{x 2}}\left[E_{1}\left\{\frac{\hat{x}^{2}+\left(z-z_{n}^{+}\right)^{2}}{4 \alpha_{2} t}\right\}-E_{1}\left\{\frac{\hat{x}^{2}+\left(2-z_{n}^{-}\right)^{2}}{4 \alpha_{2} t}\right\}\right]
$$

For a pair of horizontal ivells, the pressure distribution may be calculated by superposition,

$$
u=u_{1}+u_{2}
$$

where $u_{1}$ is the response to well 1 and $u_{2}$ is the response to well 2 . For well 1 located at $x^{\prime}=0, z^{\prime}=a$ and ivell 2 located at $x^{\prime}=0, z^{\prime}=b$, the transient pressure solution is

$$
u=\frac{q_{1}}{4 \pi \alpha_{22}} S_{1}+\frac{q_{2}}{4 \pi \alpha_{22}} S_{2}
$$

in which $q_{1}$ and $q_{2}$ are given by (13) with $\dot{M}$ replaced by $\dot{M}_{1}$ and $\dot{M}_{2}$, and

$$
\begin{gathered}
S_{1}=E_{1}\left\{\frac{\hat{x}^{2}+(z-a)^{2}}{4 \alpha_{1} t}\right\}-E_{1}\left\{\frac{\hat{x}^{2}+(z+a)^{2}}{4 \alpha_{2} t}\right\} \\
+\sum_{n=1}^{-}\left[E_{1}\left\{\frac{\hat{x}^{2}+\left(z+(-1)^{n+1} a-2 n h\right)^{2}}{4 \alpha_{2} t}\right\}+E_{1}\left\{\frac{\hat{x}^{2}+\left(z+(-1)^{n+1} a+2 n h\right)^{2}}{4 \alpha_{2} t}\right\}\right.
\end{gathered}
$$




$$
\left.-E_{1}\left\{\frac{\hat{x}^{2}+\left(z+(-1)^{n} a-2 n h\right)^{2}}{4 \alpha_{2} t}\right\}-E_{1}\left\{\frac{\hat{x}^{2}+\left(z+(-1)^{n} a+2 n h\right)^{2}}{4 \alpha_{2} t}\right\}\right]
$$

and

$$
\begin{gathered}
S_{2}=E_{1}\left\{\frac{\dot{x}^{2}+(z-b)^{2}}{4 \alpha_{2} l}\right\}-E_{1}\left\{\frac{\hat{x}^{2}+(z+b)^{2}}{4 \alpha_{2} l}\right\} \\
+\sum_{n=1}^{\infty}\left[E_{1}\left\{\frac{\hat{x}^{2}+\left(z+(-1)^{n+1} b-2 n h\right)^{2}}{4 \alpha_{2} t}\right\}+E_{1}\left\{\frac{\hat{x}^{2}+\left(z+(-1)^{n+1} b+2 n h\right)^{2}}{4 \alpha_{2} t}\right\}\right. \\
\left.-E_{1}\left\{\frac{\hat{x}^{2}+\left(z+(-1)^{n} b-2 n h\right)^{2}}{4 \alpha_{2} t}\right\}-E_{1}\left\{\frac{\hat{x}^{2}+\left(z+(-1)^{n} b+2 n h\right)^{2}}{4 \alpha_{2} l}\right\}\right]
\end{gathered}
$$

From (23), the actual pressure is found using (5),

$$
P=\sqrt{P_{a}^{2}-u}
$$

\section{Steady State Pressure Solution}

For large times, the arguments of the exponential integrals in (21) become very small, and $E_{1}$ may be approximated as [Carslaw and Jaeger, 1959]

$$
E_{1}(v)=-\gamma-\ln (v)
$$

Then for large times, (21) becomes

$$
u=\frac{q}{4 \pi \alpha_{\pi z}}\left[\ln \left\{\frac{\hat{x}^{2}+\left(2-z_{n}^{-}\right)^{2}}{\hat{x}^{2}+\left(z-z_{n}^{+}\right)^{2}}\right\}\right]
$$

or, in expanded form.

$$
u=\frac{q_{1}}{4 \pi \alpha_{x z}} \bar{S}_{1}+\frac{q_{2}}{4 \pi \alpha_{x z}} \bar{S}_{2}
$$


where

$$
\bar{S}_{1}=\ln \left\{\frac{\hat{x}^{2}+(z+a)^{2}}{\hat{x}^{2}+(z-a)^{2}}\right\}+\sum_{n=1}^{\infty} \ln \left\{\frac{\left(\hat{x}^{2}+\left(z+(-1)^{n} a-2 n h\right)^{2}\right)\left(\hat{x}^{2}+\left(z+(-1)^{n} a+2 n h\right)^{2}\right)}{\left(\hat{x}^{2}+\left(z+(-1)^{n+1} a-2 n h\right)^{2}\right)\left(\hat{x}^{2}+\left(z+(-1)^{n+1} a+2 n h\right)^{2}\right)}\right\}
$$

and

$$
\bar{S}_{2}=\ln \left\{\frac{\hat{x}^{2}+(z+b)^{2}}{\dot{x}^{2}+(z-b)^{2}}\right\}+\sum_{n=1}^{\infty} \ln \left\{\frac{\left(\hat{x}^{2}+\left(z+(-1)^{n} b-2 n h\right)^{2}\right)\left(\hat{x}^{2}+\left(z+(-1)^{n} b+2 n h\right)^{2}\right)}{\left(\hat{x}^{2}+\left(z+(-1)^{n+1} b-2 n h\right)^{2}\right)\left(\dot{x}^{2}+\left(z+(-1)^{n+1} b+2 n h\right)^{2}\right)}\right\}
$$

The solution given by (29) may be written in dimensionless form by defining

$$
\theta=\frac{P^{2}}{P_{a}^{2}}=1-\frac{u}{P_{a}^{2}}
$$

Then

$$
\theta=1-\left(\xi_{1} \bar{s}_{1}+\xi_{2} \bar{S}_{2}\right)
$$

where

$$
\xi_{1}=\frac{\mu \dot{M}_{1}}{2 \pi \rho_{a} P_{a} \sqrt{k_{x} k_{2}}}
$$

and

$$
\xi_{2}=\frac{\mu \dot{M}_{2}}{2 \pi \rho_{a} P_{a} \sqrt{k_{x} k_{2}}}
$$

In (34) and (35), $\rho_{0}$ is a reference gas density calculated using the ideal gas law with a pressure of $P_{a}$ and an absolute temperature of $T$. In some instances, it is more convenient to write (34) and (35) in tenns of constant volumetric gas flowrates per unit length of screen. In this case, the quantities $\dot{M}_{1} / \rho_{a}$ and $\dot{M}_{2} / \rho_{a}$ in (34) and (35) are replaced by the volumetric gas tlowrates per unit screen length, $q_{a 1}$ and $q_{a 2}$ where both 
$q_{a 1}$ and $q_{a 2}$ are measured at a pressure of $P_{a}$. With this formulation, the pressure solution is independent of gas composition.

\section{Streamfunction Solution}

Using the steady state pressure distribution given by (33), the strearnfunction solution is obtained by applying the Cauchy-Reimann equations:

$$
\frac{\partial \psi}{\partial z}=\frac{\partial \theta}{\partial \dot{x}}
$$

and

$$
\frac{\partial \psi}{\partial \hat{x}}=-\frac{\partial \theta}{\partial z}
$$

Using (36),

$$
\dot{\psi}=\int \frac{\partial \theta}{\partial \dot{x}} d z
$$

Since

$$
\frac{\partial \theta}{\partial \dot{x}}=-\xi_{1} \frac{\partial \bar{S}_{1}}{\partial \hat{x}}-\xi_{2} \frac{\partial \bar{S}_{2}}{\partial \hat{x}}
$$

the streamfunction is determined by evaluating

$$
\begin{gathered}
\int \frac{\partial \theta}{\partial \hat{x}} d z=-\xi_{1}(2 \hat{x}) \int\left[\frac{1}{\hat{x}^{2}+(z+a)^{2}}-\frac{1}{\hat{x}^{2}+(z-a)^{2}}\right. \\
+\sum_{n=1}^{\infty}\left[\frac{1}{\hat{x}^{2}+\left(z+(-1)^{n} a-2 n h\right)^{2}}+\frac{1}{\hat{x}^{2}+\left(z+(-1)^{n} a+2 n h\right)^{2}}\right. \\
\left.\left.-\frac{1}{\hat{x}^{2}+\left(z+(-1)^{n+1} a-2 n h\right)^{2}}-\frac{1}{\hat{x}^{2}+\left(z+(-1)^{n+1} a+2 n h\right)^{2}}\right]\right] d z
\end{gathered}
$$




$$
\begin{gathered}
-\xi_{2}(2 \hat{x}) \int\left[\frac{1}{\hat{x}^{2}+(z+b)^{2}}-\frac{1}{\hat{x}^{2}+(z-b)^{2}}\right. \\
+\sum_{n=1}^{\infty}\left[\frac{1}{\hat{x}^{2}+\left(z+(-1)^{n} b-2 n h\right)^{2}}+\frac{1}{\hat{x}^{2}+\left(z+(-1)^{n} b+2 n h\right)^{2}}\right. \\
\left.\left.-\frac{1}{\hat{x}^{2}+\left(z+(-1)^{n+1} b-2 n h\right)^{2}}-\frac{1}{\hat{x}^{2}+\left(z+(-1)^{n+1} b+2 n h\right)^{2}}\right]\right] d z
\end{gathered}
$$

Each of the integrals in (40) may be written in the form of

$$
\int \frac{d z}{\hat{x}^{2}+\left(z+z_{n}\right)^{2}}=\int \frac{d z}{A+B z+C z^{2}}
$$

where $z_{n}=z_{n}^{ \pm}, A=z_{n}^{2}+\dot{x}^{2}, B=2 z_{n}$, and $C=1$. For

$$
p=4 A C-B^{2}=4 \hat{x}^{2}>0
$$

the integral is given by Beyer [1981],

$$
\int \frac{d z}{A+B z+C z^{2}}=\frac{2}{\sqrt{p}} \arctan \left\{\frac{2 C z+B}{\sqrt{p}}\right\}
$$

or

$$
\int \frac{d z}{\hat{x}^{2}+\left(z+z_{n}\right)^{2}}=\frac{1}{\hat{x}} \arctan \left\{\frac{z+z_{n}}{\hat{x}}\right\}
$$

Therefore, the streamfunction is

$$
\begin{gathered}
\psi=-2 E_{1}\left\{\arctan \left\{\frac{2+a}{\hat{x}}\right\}-\arctan \left\{\frac{2-a}{\hat{x}}\right\}\right. \\
+\sum_{n=1}^{-}\left[\operatorname{arctun}\left\{\frac{2+(-1)^{n} a-2 n h}{\hat{x}}\right\}+\arctan \left\{\frac{2+(-1)^{n} a+2 n h}{\hat{x}}\right\}\right. \\
\left.\left.-\arctan \left\{\frac{2+(-1)^{n+1} a-2 n h}{\hat{x}}\right\}-\arctan \left\{\frac{2+(-1)^{n+1} a+2 n h}{\hat{x}}\right\}\right]\right]
\end{gathered}
$$




$$
\begin{aligned}
& -2 \xi_{2}\left\{\arctan \left\{\frac{z+b}{\hat{x}}\right\}-\arctan \left\{\frac{z-b}{\hat{x}}\right\}\right. \\
& +\sum_{n=1}^{-}\left[\arctan \left\{\frac{2+(-1)^{n} b-2 n h}{\hat{x}}\right\}+\arctan \left\{\frac{z+(-1)^{n} b+2 n h}{\hat{x}}\right\}\right. \\
& \left.\left.-\arctan \left\{\frac{z+(-1)^{n+1} b-2 n h}{\hat{x}}\right\}-\arctan \left\{\frac{2+(-1)^{n+1} b+2 n h}{\hat{x}}\right\}\right]\right]
\end{aligned}
$$

In the streamfunction solution, (45), the integration constant resulting from the integration in (38) is found to be zero after substituting (45) into the second Cauchy-Reimann equation, (37). Complete listings of the FORTRAN programs used to evaluate the steady state pressure and streamfunction solutions are given in Listings 1 and 2 , respectively.

\section{APPLICATIONS}

In this section, several examples are given to show the effects of well flowrates and porous media anisotropy on the steady state gas pressure and streamfunction fields. The steady state pressure solution is also compared with results generated by a integral finite difference numerical sinulator. In each of these examples, the thickness of the unsaturated zone, $h$ is $20 \mathrm{~m}$, the depth to the upper well, $a$ is $9.5 \mathrm{~m}$, and the depth to the lower well, $b$ is $17.5 \mathrm{~m}$. For each case, the ambient pressure, $P_{a}$ is 101325 pascals, the temperature, $T$ is $10^{\circ} \mathrm{C}$, the ambient gas density, $\rho_{a}$ is $1.248 \mathrm{~kg} / \mathrm{m}^{3}$, and the gas viscosity, $\mu$ is $1.76 \times 10^{-5} \mathrm{~kg} / \mathrm{ms}$. 


\section{Equal Injection and Production Flowrates}

Figure 3 shows the steady state gas pressure field resulting from gas injection in the lower well at a mass rate per unit screen length of $0.02 \mathrm{~kg} / \mathrm{ms}$ with simultaneous gas production from the upper well at the same rate. For this example, the effective gas permeability is isotropic, and equal to $2 \times 10^{-11} \mathrm{~m}^{2}$. The contour interval is 0.01 atmospheres, and the contours surrounding the upper well represent gas pressure drawdown due to gas extraction, while those surrounding the lower well represent gas pressure buildup due to gas injection. The upper surface is held constant at a pressure of one atmosphere, and a contour of pressure equal to one atmosphere extends downward from the ground surface to a location between the two wells.

The corresponding streamfunction distribution is shown in Figure 4. Each of the streamtubes in this figure represent 5 percent of the gas flow (on a mass basis). Because the magnitude of the well flowrates is equal, there are 20 streamtubes entering the upper well, and 20 streamtubes leaving the lower well. From this streamfunction distribution, it is apparent that not all of the gas injected into the lower well is recovered by the upper extraction well. In fact, about 12-13 percent of the gas injected into the lower well escapes the capture zone of the upper well, and eventually exits the system through the ground surface. Similarly, about 12-13 percent of the gas entering the upper well originates at the ground surface, and not at the injection well. Between the two wells, the streamtubes are very narrow, indicating very high gas flows in this region.

For gas injection and production in an isotropic system. Figures 3 and 4 show that the zone of influence due to gas pumping is fairly small, and restricted to a horizontal 
distance of roughly 1-1.5 times the thickness of the unsaturated zone. In an anisotropic system, the induced gas pressure and streamfunction distributions may be quite different than those in an isotropic system. In Figure 5, the gas pressure distribution is shown for an anisotropic case which is identical to that considered above, except that the vertical gas permeability, $k_{2}$ has been reduced by a factor of 10 to a value of $2 \times 10^{-12} \mathrm{~m}^{2}$. As in Figure 3, the gas pressure contour interval is 0.01 atmospheres, and the upper surface is held at a constant pressure of one atmosphere. Clearly, the zone of gas pressure intluence in Figure 5 is much larger than that in the isotropic permeability case.

The corresponding streamfunction distribution for this anisotropic example is shown in Figure 6, and in comparison with the isotropic case (Figure 4), the gas flow field extends over a much larger horizontal distance. For this anisotropic example, the streamfunction distribution is "stretched" by a factor of $(10)^{1 / 2}$ relative to the isotropic case in accordance with the horizontal coordinate transformation given by (17). Although the shape of the streamlines in Figure 6 are different from those in Figure 4, the same amount of injected gas escapes the capture zone of the upper well, about 12-13 percent.

For the removal of contaminants in the unsaturated zone by a pair of horizontal wells, it is clear that gas should not be injected into the lower well at a rate equal to that in the upper extraction well. To do so may result in the migration of contaminants away from the lower well, out of the capture zone of the upper well. 


\section{Unequal Injection and Production Flowrates}

For operations in which the magnitude of injection and production flowrates are different, the gas flowfield is substantially different from the cases considered above. Figure 7 shows the steady state pressure field resulting from gas injection at a mass rate per unit screen length of $0.01 \mathrm{~kg} / \mathrm{ms}$ in the lower well with gas production at a rate of $0.02 \mathrm{~kg} / \mathrm{ms}$ in the upper well. For this example, the system is isotropic with a permeability of $2 \times 10^{-i l}$, and the contour interval is 0.01 atmosphere with a constant pressure of one atmosphere at the ground surface. Due to the larger rate of gas removal in the upper well relative to the rate of gas injection in the lower well, the pressure field is dominated by the influence of the extraction well, and only a small region around the lower ivell is subject to a relative pressure buildup.

The difference between this type of operation and that described in the previous section is more clearly illustrated by the streamfunction distribution for this case, shown in Figure 8. Each of the streamtubes in this contour plot account for five percent (on a mass basis) of the total flow to the upper (extraction) well. In this situation, all of the gas injected into the lower well is recovered by the extraction well, and the capture zone for the injected gas is represented by the streamline which originates at the lower boundary at $x / h$ equal to about 0.6 and extends to the upper well. As would be expected, 50 percent of the gas entering the extraction well originated at the injection well, while the remaining 50 percent originates at the ground surface. The region between the two wells is subject to a high rate of gas flow as evidenced by the large number of streamiubes in this area. 
While the gas flow field in this isotropic example is limited in horizontal extent to scaled distances $(x / h)$ of a little over one, in anisotropic media, the flow field extends over a much larger range. This is seen in Figure 9 which shows the pressure distribution (with a contour interval of 0.01 atmosphere) for a case identical to that shown in Figure 7, but with a reduction of the vertical gas permeability by a factor of 10 to a value of $2 \times 10^{-12} \mathrm{~m}^{2}$. Compared with the isotropic example, the region of influence, and the magnitude of pressure drawdown and buildup are much larger in this case.

The streamfunction distribution for this example is shown in Figure 10. As in the last isotropic example (Figure 8), all of the gas injected into the lower well is captured by the upper well. The location of the injected gas capture zone is represented in this figure by the streamline extending from the lower boundary at a scaled horizontal distance of about 1.9 to the extraction well. Again, 50 percent of the gas entering the upper well originated from the lower well, and the remaining $\mathbf{5 0}$ percent originates at the ground surface. The streanfunction distribution for this anisotropic case is identical to that obtained for the isotropic case except that the streamlines are "stretched" by a factor of $(10)^{1 / 2}$ in the horizontal dimension.

In Figure 10, (and the earlier streamfunction plots) it is apparent that the zone between the two wells is swept by large amounts of gas. This suggests that the two-well injection/extraction configuration may be particularly effective at removing contaminants located in deeper parts of the unsaturated zone. For comparison, Figure 11 shows the streamfunction distriburion due to gas extraction from the upper well with no gas injection in the lower well. In this case, the amount of gas flowing through the lower part of the unsaturated zone is much smaller than in the other examples. 


\section{Comparison with Numerical Solution}

As an independent check of the analytical steady state pressure solution, the anisotropic example shown in Figures 9 and 10 was modeled numerically using the multiphase integral finite difference simulator TOUGH2 [Pruess, 1991]. The simulation was performed using a fine mesh consisting of 20 elements in the vertical direction and 50 elements in the horizontal direction. Due to symmetry, only half of the system was modeled (see, for example, Figures 3 through 11), and the horizontal mesh spacing was very tine near the well, with an increasing spacing away from the well. Because TOUGH 2 models transient problems, the boundary and well conditions were applied to a uniform initial pressure field, and the simulator was allowed to run until the gas pressure field showed no change with respect to time.

A comparison of the analytically and numerically generated pressure distribution along a vertical line at $x=1.25 \mathrm{~m}(x / h=1) .0625)$ is shown in Figure 12. The results are in excellent agreement and show the strong pressure drawdown near the upper well (located at $z=9.5 \mathrm{~m}$ ) and the pressure buildup near the lower well (located at $z=17.5 \mathrm{~m}$ ).

The analytically and numerically determined pressure distributions along a horizontal line through the lower well are compared in Figure 13. Again, the results are in good agreement except very close to the well where the numerical simulator underpredicts the amount of pressure buildup. This is due to the fact that in the analytical solution, the injection well is modeled as a line source, while in the numerical model, gas is injected into a rectanguiar volume element. The reversal of the gas pressure gradient at a distance of about $35 \mathrm{~m}(x / \mathrm{h}=1.75)$ is indicative of the gas capture zone of the upper extraction well. 


\section{SUMMARY}

Analytical solutions are presented for transient and steady state gas flow to a pair of parallel horizontal wells in the unsaturated zone. The porous media is assumed to be homogeneous but may be anisotropic, and the well system is modeled as a twodimensional cross-section through the wells, neglecting end effects.

Based on the results of this study, several conclusions may be drawn. Firstly, in a horizontal two-well gas injection/extraction system, it is important to produce gas at a rate substantially larger than the rate of gas injection. This must be done to ensure that all of the injected gas is captured by the extraction well so that contaminants are not transported away from the site by the injected gas.

Secondly, it is apparent that horizontal well flow systems are very strongly affected by gas permeability anisotropy. Neglecting this effect could result in large errors in the gas pressure and streamfunction distributions. Finally, a comparison of the streamfunction distribution for two-well injection/extraction systems with the streamfunction distribution for single-well extraction systems indicates that the twowell system may be very effective for removing contaminants located in deeper parts of the unsaturated zone.

In this initial study, several limiting assumptions were made. Perhaps the most restrictive of these is the assuniption that the porous media is homogeneous. Real systerns are likely to be heterogeneous at least to some degree, and in many cases, this permeability heterogeneity will in large part determine the gas flowfield. Also, the assumption that the induced gas flowtield is two-dimensional may result in significant errors in problems where the length of the horizontal well screens are short compared 
to the thickness of the flow system. In these instances, the gas flowfield will be fully three-dimensional, and the present solutions are not applicable. 
program horizp

c

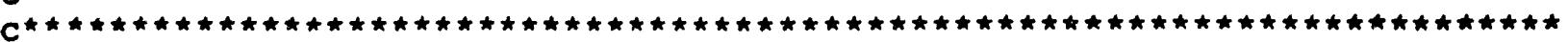

C C

c.

This program evaluates the analytical pressure field solution for flow due to the steady state pumping of two horizontal gas wells. The two wells may be pumped at different rates, and may be injecting or producing gas. Porous media may be anisotropic. Solution and program developed by Ron Falta 7/24/92.

-Input variables

$h=$ depth to impermeable boundary

$a=$ scaled $z$ coordinate of upper well = depth/h

$b=$ scaled $z$ coordinate of lower well $=$ depth $/ h$

c1 = dimensionless source strength of upper well

$=m u \star M I /\left(2 .{ }^{\star} p i{ }^{\star} r h o g \star P a \star \operatorname{sqrt}(K x \star K z)\right)$

where

mu $=$ gas viscosity, Pascal*s

$M 1$ = gas mass injection/production rate per unit length of screen (positive for production), $\mathrm{kg} / \mathrm{s}$

rhog $=$ ambient gas density, $\mathrm{kg} / \mathrm{m} \star \star 3$

$\mathrm{Pa}=$ ambient gas pressure, pascals

$K x=$ effective horizontal gas permeability, $m \star \star 2$

$k z=$ effective vertical gas permeability, $m \star \star 2$

c2 = dimensionless source strength of lower well

$=m u{ }^{\star}\left[12 /\left(2 .{ }^{*} p i{ }^{\star} r h o g{ }^{*} P a{ }^{\star} \operatorname{sqrt}\left(K x{ }^{\star} K z\right)\right)\right.$

where

mu $=$ gas viscosity, Fascal*s

$H 2$ = gas mass injection/production rate per unit length of screen (positive for production), $\mathrm{kg} / \mathrm{sm}$

rhog = ambient gas density, $\mathrm{kg} / \mathrm{m} \star \star 3$

$\mathrm{Pa}=$ ambient gas pressure, pascals

$K x=$ effective horizontal gas permeabil1ty, $m * \star 2$

$K z=$ effective vertical gas permeability, $m * \star 2$

note that $M /$ rhog in $c 1$ and $c 2$ may be replaced by the volumetric flowrates at a pressure of $\mathrm{Pa}, \mathrm{Q} 1$ and $\mathrm{Q} 2$.

aratio = ratio of vertical to horizontal gas permeability $=K z / K x$

$k=$ number of points to be calculated in $x$ direction:

$l=$ number of points to be calculated in $z$ direction.

$x(i)=$ scaled $x$-coordinate $=x / h$ 


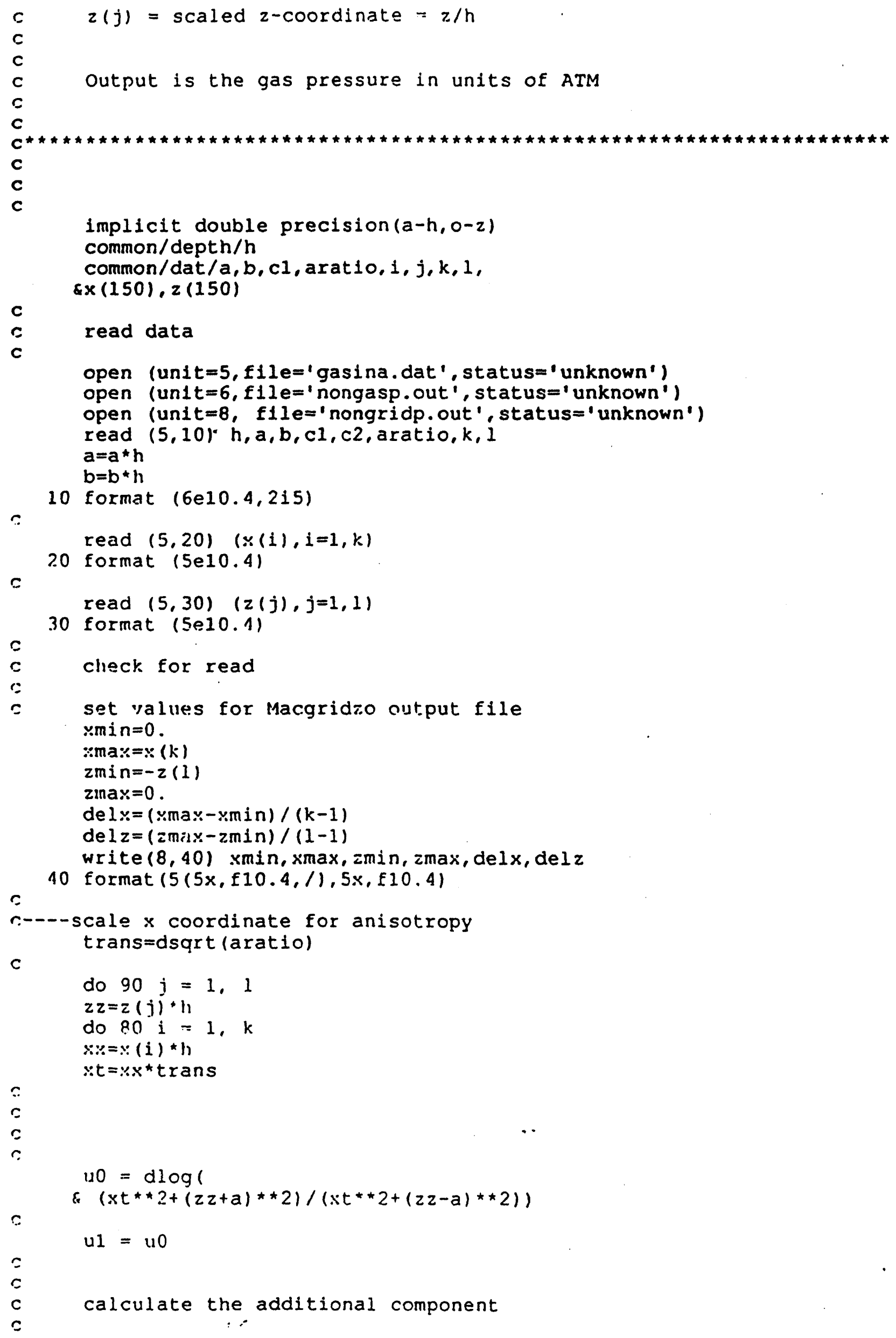


$n=0$

70 continue

$n=n+1$

C

un $=d \log ($

s $(x t \star \star 2+(z z+(-1) \star \star n \star a-2 \star n \star h) \star \star 2) \star$

c $(x t * \star 2+(z z+(-1) \star * n \star a+2 * n \star h) \star \star 2) /$

c $((x t * * 2+(z z+(-1) * \star(n+1) \star a-2 * n * h) * \star 2) *$

$c$

* $(x t \star \star 2+(z z+(-1) \star \star(n+1) \star a+2 * n \star h) \star \star 2)))$

$u 1=u 1+u n$

$c$

if (dabs (un) .gt.0.00001) then

goto 70

end $1 \mathrm{f}$

$c$

$u 0=d \log ($
$\varepsilon(x t \star 2+(z z+b) * \star 2) /(x t * \star 2+(z z-b) \star \star 2))$

C

$u_{2}=u_{0}$

c

$c$

C

c

calculate the additional component

$n=0$

72 continue

$n=n+1$

$c$

$u n=d \log ($

\& $(x t * \star 2+(z z+(-1) * * n * b-2 * n * h) * \star 2) *$

\& $(x t * 2+(z z+(-1) * * n * b+2 * n * h) * \star 2) /$

*. $((x t * 2+(z z+(-1) * *(n+1) * b-2 * n * h) * * 2) *$

* $(x t * \star 2+(z z+(-1) * *(n+1) * n+2 * n * n) * \star 2)))$

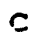

$112=u 2+u n$

$=$

if (dabs (un).gt.0.00001) then

goto 7 ?

endif

c

thet $a=1 .-(c 1 * u 1+c 2 \star u 2)$

$c$

$c$

c

c-----covert from dimensionless $p^{\star \star 2}$ to atm

C

if (theta.lt.0.) theta $=0$.

pu=dsqrt $($ theta)

$\mathrm{zz} 1=-\mathrm{zz} / \mathrm{h}$

$\times \times 1=\times \times / h$

if (i.eq. 1$) \times \times 1=0.0$

c-1-- The 3 column output nongasp. out is disabled for now

$c$

write $(6,120) \quad x: x 1, z 21, p u$

120 format $(3(5 x$, F 12.6$))$

write $(8,121)$ pu

121 format $(5 x, F 12.6)$

c

80 continue

90 continue

$c$

return

end 
LISTING 2

program horizs

c C

This program evaluates the analytical streamfunction solution for flow due to the steady state pumping of two horizontal gas wells. The two wells may be pumped at different rates, and may be infecting or producing gas. The media may be anisotropic. Solution and program developed by Ron Falta 7/24/92.

$h=$ depth to impermeable boundary. $m$

$a=$ scaled $z$ coordinate of upper well = depth/h

$b=$ scaled $z$ coordinate of lower well = depth/h

$r_{1}=$ dimensionless source strength of upper well

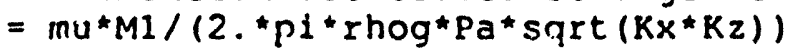

where

$m u=g a s$ viscosity

$M 1$ = gas inass injection/production rate per unit length of screen (positive for production), $\mathrm{kg} / \mathrm{sm}$

rlog $=$ ambient gas density, $\mathrm{kg} / \mathrm{m} \star 3$

$\mathrm{Pa}=$ ambient gas pressure, $\mathrm{Pa}$

$K_{:}:=$effective horizontal gas permeability, $m * \star 2$

$K z=$ effective vertical gas permeability, $m * \star 2$

$c 2$ = dimensionless source strength of lower well

$=m u{ }^{\star} M 2 /\left(2 .{ }^{\star} p i{ }^{\star} \mathrm{rhog}^{\star} \mathrm{Pa}{ }^{\star} \operatorname{sqrt}\left(K{ }^{\star}{ }^{*} \mathrm{Kz}\right)\right)$

where

mu $=$ gas viscosity

$M 2$ = gas mass injection/production rate per unit length of screen (positive for production), $\mathrm{kg} / \mathrm{s}$

rhog $=$ ambient gas density, $\mathrm{kg} / \mathrm{m} \star \star 3$

$\mathrm{Pa}=$ ambient gas pressure, Pascals

$\mathrm{Kx}=$ effective horizontal gas permeability, $m * * 2$

$K z=$ effective vertical gas permeability, $m \star \star 2$

note that $M /$ rhog in $c 1$ and $c 2$ may be replaced by the volumetric flowrates t a pressure of $\mathrm{Pa}, \mathrm{Q} 1$ and $\mathrm{Q} 2$. $\begin{aligned} \text { aratio } & =\text { ratio of vertical permibility to horizontal permeabllity } \\ & =k z / K x\end{aligned}$

$k$ = number of points to be calculated in $\mathrm{x}$ direction;

1 = number of points to be calculated in $z$ direction.

$\therefore(i)=$ scaled $x$-coordinate $=x / h$ 


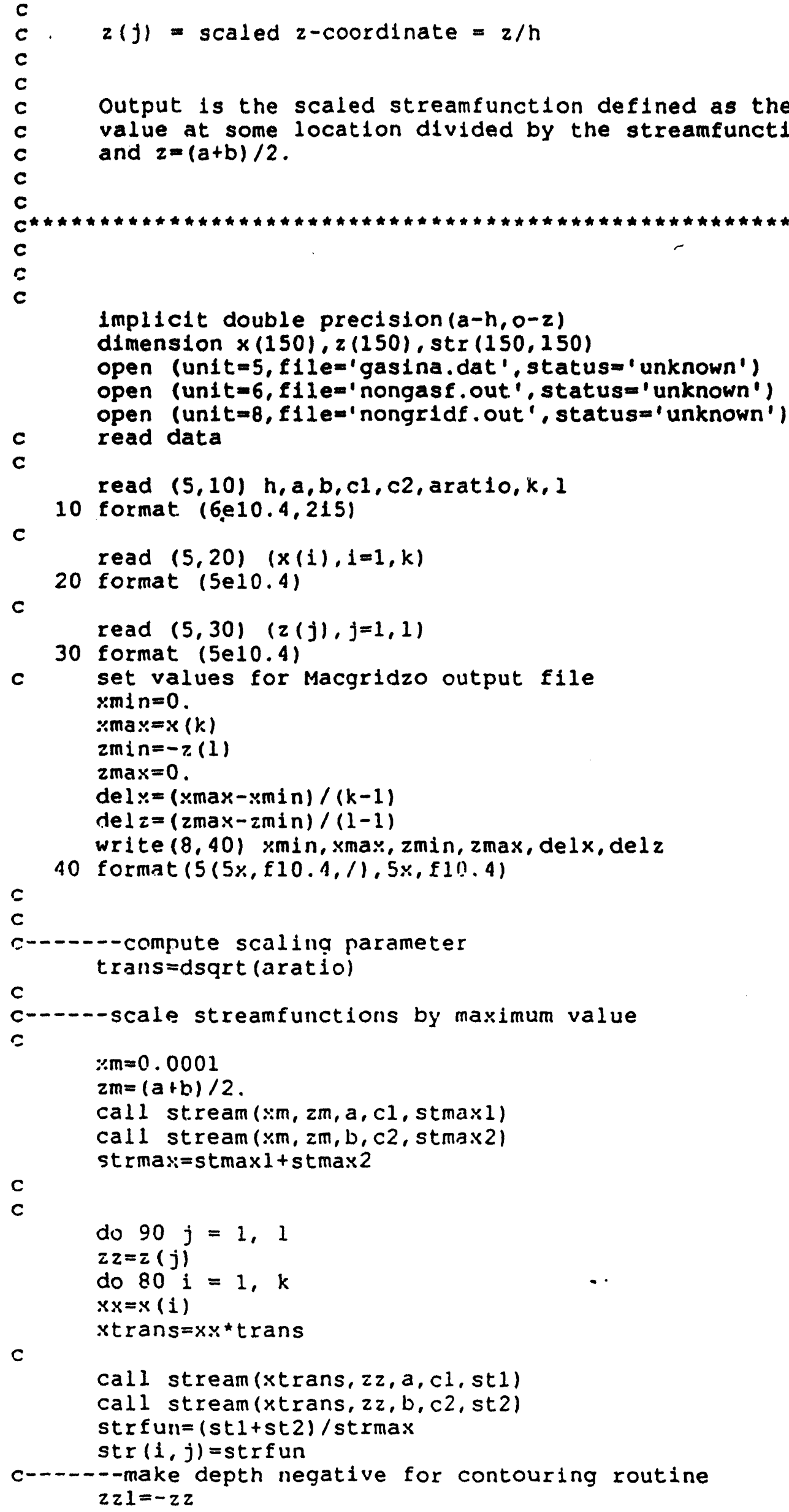

c

do $90 j=1,1$

$z z=\Sigma(j)$

do $80 i=1, k$

$x \mathrm{x}=\mathrm{x}(1)$

$x$ trans $=x * \star$ trans

C

call stream (xtrans, zz, a, cl, st 1 )

call stream (xtrans, zz, b, c2, st 2 )

strfun= (st $1+$ st 2$) /$ strmax

$\operatorname{str}(i, j)=$ strfun

c-- - - make depth negative for contouring routine $z z I=-z z$ 


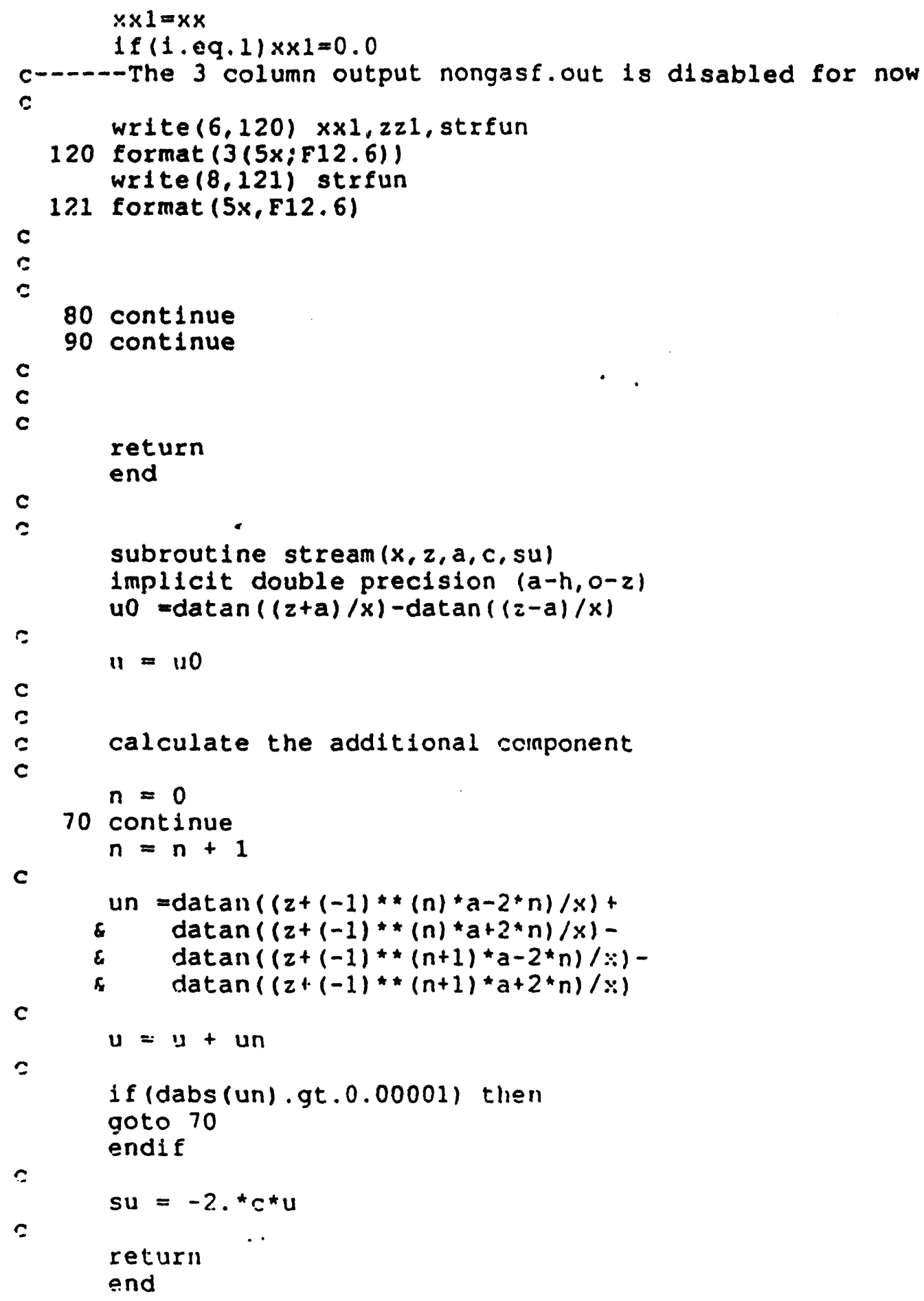




\section{ACKNOWLEDGMENTS}

The author appreciates the collaboration of managers and technical staff of the Westinghouse Savannah River Company in this effort. This work was funded by the Office of Technology Development, U.S. Department of Energy under contracts DEAC03-76SF00098 and W-7405-ENG-48. The author would like to acknowledge B.D. Hestir for preparing most of the figures in this paper, and K. Pruess and D.A. Chesnut for reviewing this manuscript. 


\section{NOTATION}

a depth to upper well, $m$.

b depth to lower well, $m$.

$h$ depth to water table or impermeable stratum, $m$.

$\ddot{k} \quad$ gas phase permeability tensor, $m^{2}$.

$k_{x} \quad$ horizontal gas phase permeability, $m^{2}$.

$k_{y} \quad$ horizontal gas phase permeability, $m^{2}$.

$k_{2} \quad$ vertical gas phase permeability, $m^{2}$.

$\dot{M} \quad$ gas mass flow rate per unit well screen length (positive for extraction), $\mathrm{kg} / \mathrm{ms}$.

$\dot{M}_{1} \quad$ upper well gas mass fiow rate per unit well screen length (positive for extrac- ' tion), $\mathrm{kg} / \mathrm{ms}$.

$\dot{M}_{2} \quad$ lower well gas mass flow rate per unit well screen length (positive for extraction), $\mathrm{kg} / \mathrm{ms}$.

$M_{m} \quad$ gas average molecular weight, g /mole.

$n \quad$ summation index.

P gas pressure, Parcals.

$P_{a} \quad$ ambient gas pressure. Pascals.

$q$ continuous gas source strength per length of well screen, $\mathrm{kg}^{2} / \mathrm{s}^{5}$.

$q_{1} \quad$ upper well continuous gas source strength per length of well screen, $\mathrm{kg}^{2} / \mathrm{s}^{5}$.

$q_{2}$ lower well continuous gas source strength per length of well screen, $\mathrm{kg}^{2} / \mathrm{s}^{5}$. 
$q_{a 1} \quad$ upper well volumetric gas flowrate per unit screen length at a pressure of $P_{a}$, $m^{3} / m s$.

Qa2 lower well volumetric gas flowrate per unit screen length at a pressure of $P_{a}$, $m^{3} / m s$.

$Q \quad$ instantaneous point or line source strength; for point source, $\mathrm{kg}^{2} \mathrm{~m} / \mathrm{s}^{4}$; for line source, $\mathrm{kg}^{2} / \mathrm{s}^{4}$.

$Q_{\text {in }} \quad$ total volumetric gas injection flowrate at a pressure of $P_{a}, m^{3} / s$.

Qous total volumetric gas extraction flowrate at a pressure of $P_{a}, \mathrm{~m}^{3} / \mathrm{s}$.

$r \quad$ defined by (14).

$R \quad$ universal gas constant, $m J / m o l e K$.

$S_{1} \quad$ defined by $(24)$.

$S_{2} \quad$ detined by (25).

$\bar{S}_{1} \quad$ defined by (30).

$\bar{S}_{2} \quad$ defined by $(31)$.

t time, s.

$T \quad$ temperature, $K$.

$1 \quad$ pressure difference variable defined by (5), $\mathrm{kg}^{2} / \mathrm{m}^{2} \mathrm{~s}^{4}$.

$x$ horizontal distance from wells, $m$.

$\hat{x} \quad$ transformed horizontal distance from wells defined by (17), $m$.

$z \quad$ depth from ground surface, $m$. 
$z_{n}^{-} \quad$ depth from ground surface to line source, $m$.

$z_{n}^{+} \quad$ depth from ground surface to line sink, $m$.

$\alpha_{z} \quad$ effective gas phase diffusivity defined by (15), $\mathrm{m}^{2} / \mathrm{s}$.

$\alpha_{2} \quad$ vertical gas phase diffusivity defined by (16), $\mathrm{m}^{2} / \mathrm{s}$.

$\mu \quad$ gas phase viscosity, Pascal s.

@ gas filled porosity (volumetric gas content).

$\psi \quad$ streamifunction.

P. ambient gas phase density, $\mathrm{kg} / \mathrm{m}^{3}$.

$\theta$ dimensionless pressure squared, defined by (32).

$\xi_{1}$ dimensionless upper well gas line sink strength defined by (34).

$\zeta_{2}$ dimensionless lower well gas line sink strength defined by (35). 


\section{REFERENCES}

Baehr, A. L. and M. F. Hult, Evaluation of unsaturated zone air permeability through pneumatic tests, Water Resources Research, 27(10), 2605-2617, 1991.

Bear, J., Dynamics of Fluids in Porous Media, American Elsevier, New York, NY, 764 pp., 1972.

Bear, J., Hydraulics of Groundwater, McGraw-Hill, New York, NY, 569pp., 1979.

Carslaw, H. S. and J. C. Jaeger, Heat Conduction in Solids, Oxford University Press, New York, 1959.

Collins, R. E., Flow of Fluids Through Porous Materials, Reinhold Publishing Corporation, New York, NY, 270 pp., 1961.

Johnson, P. C., M. W. Kemblowski, and J. D. Colthart, Practical screening models for soil venting applications, Proceedings of Petroleum Hydrocarbons and Organic Chemicals in Ground Water, Natl. Water Well Assoc., Houston, TX, 521-546, 1988.

Johnson, P. C., M. W. Kemblowski, and J. D. Colthart, Quantitative analysis for the cleanup of hydrocarbon-contaminated soils by in-situ soil venting, Ground Water, 28(3), 413-429, 1990)a.

Johnson, P. C., C. C. Stanley, M. W. Kemblowski, D. L. Byers, and J. D. Colthart, A practical approach to the design, operation, and monitoring of in situ soil-venting systems, Ground Water Monit. Rev., 10(2), 159-178, $1990 \mathrm{~b}$.

Kidder, R. E., Unsteady flow of gas through a semi-infinite porous medium, J. of Appl. Mech. Trans., ASME, 24(3), 329-332, 1957. 
Klinkenberg, L. J., The permeability of porous media to liquids and gases, Drilling and Production Practice, API, 200-213, 1941.

Looney, B. B., T. C. Hazen, D. S. Kaback, and C. A. Eddy, Full scale field test of the in situ air stripping process at the Savannah River Integrated Test Site, Draft Report WSRC-RD-91-22, Westinghouse Savannah River Company, June, 1991.

Massmann, J. W., Applying groundwater flow models in vapor extraction system design, J. Environ. Eng., 115(1), 129-149, 1989.

McWhorter, D. B., Unsteady radial flow of gas in the vadose zone, J. Contam. Hydrol., $5,297-314,1990$.

Pruess, K., TOUGH2-a general-purpose numerical simulator for multiphase fluid and heat flow, Lawrence Berkeley Laboratory Report LBL-29400 UC-251, May, 1991.

Shan, C., R. W. Falta, and I. Javandel, Analytical solutions for steady state gas flow to a soil vapor extraction well, Water Resources Research, 28(4), 1105-1120, 1992.

Wilson, D. J., A. N. Clarke, and J. H. Clarke, Soil clean up by in-situ aeration. 1. Mathematical modeling, Sep. Sci. and Tech., 23(10), 991-1037, 1988. 


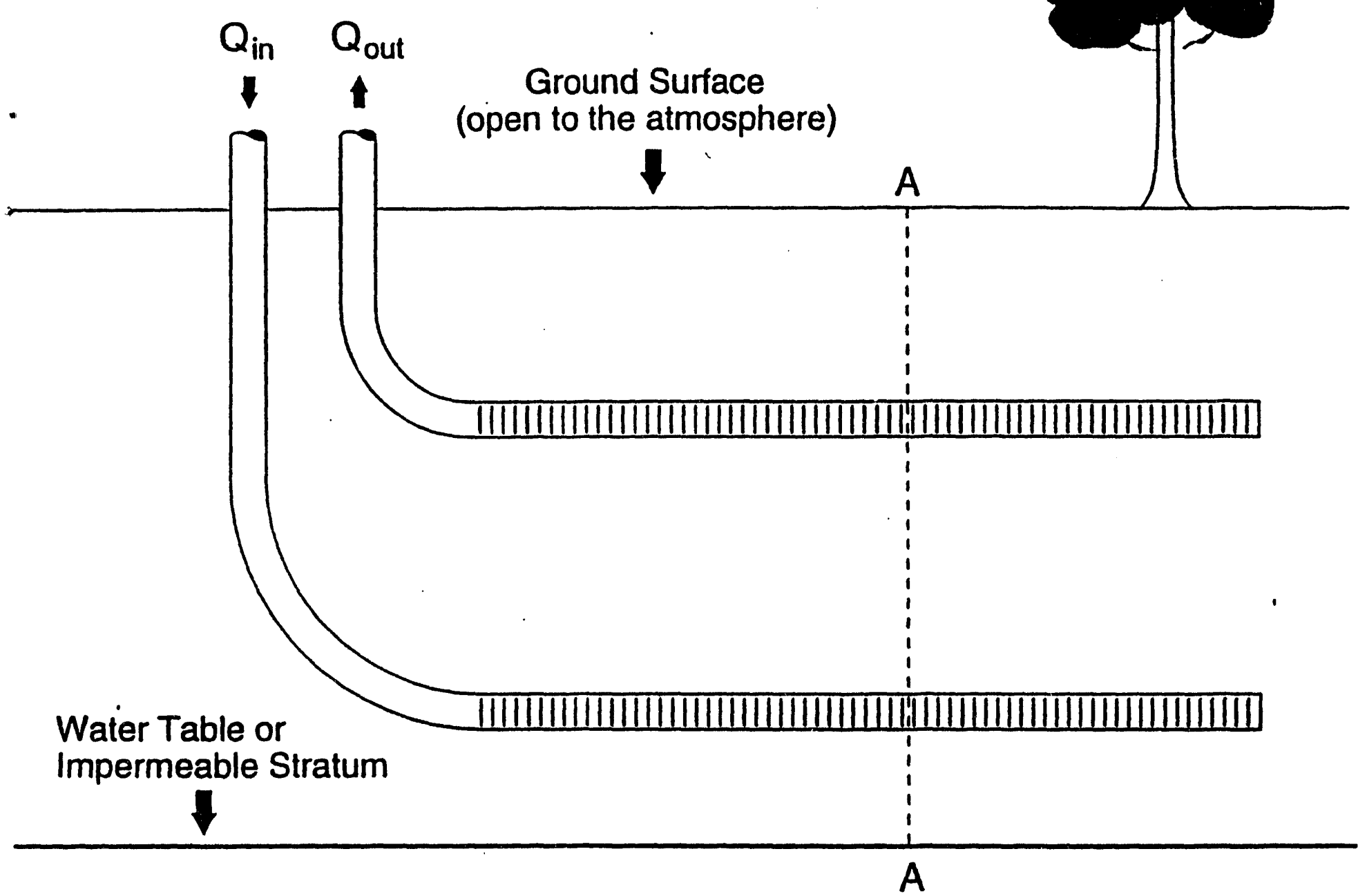

Figure 1. Side view of a horizontal well system in the unsaturated zone. 


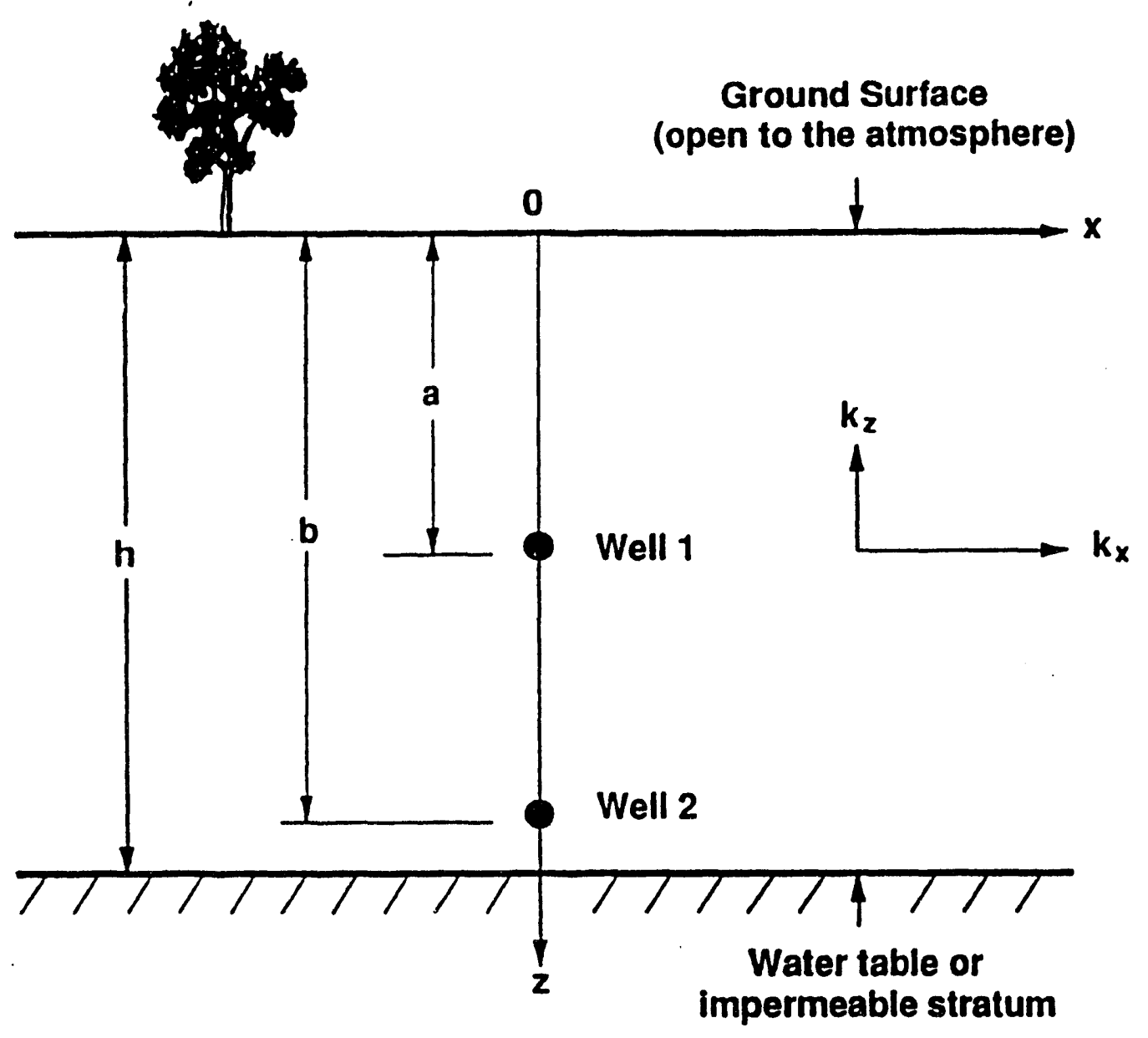

Figure 2. Cross-sectional view of a horizontal well system. 


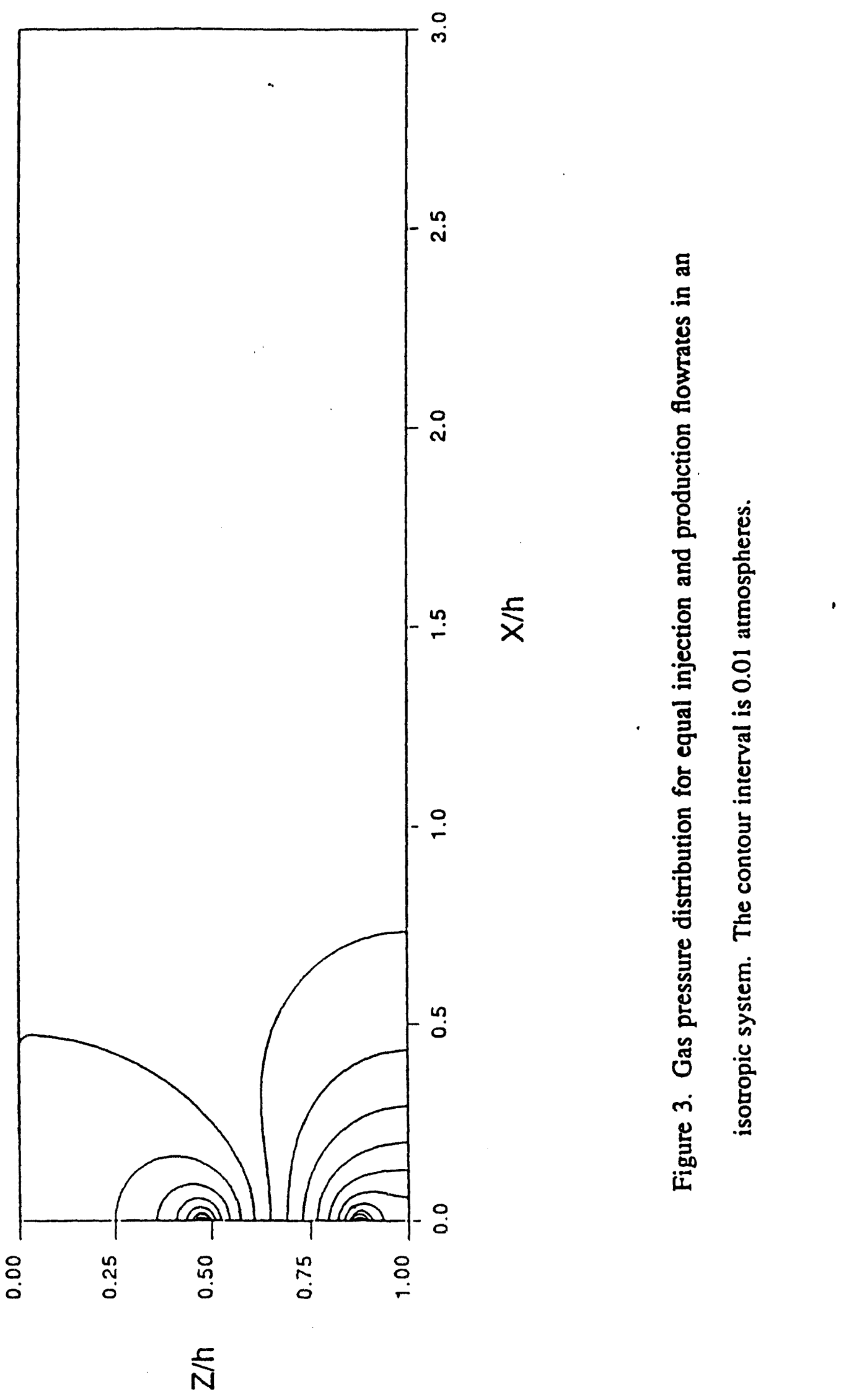




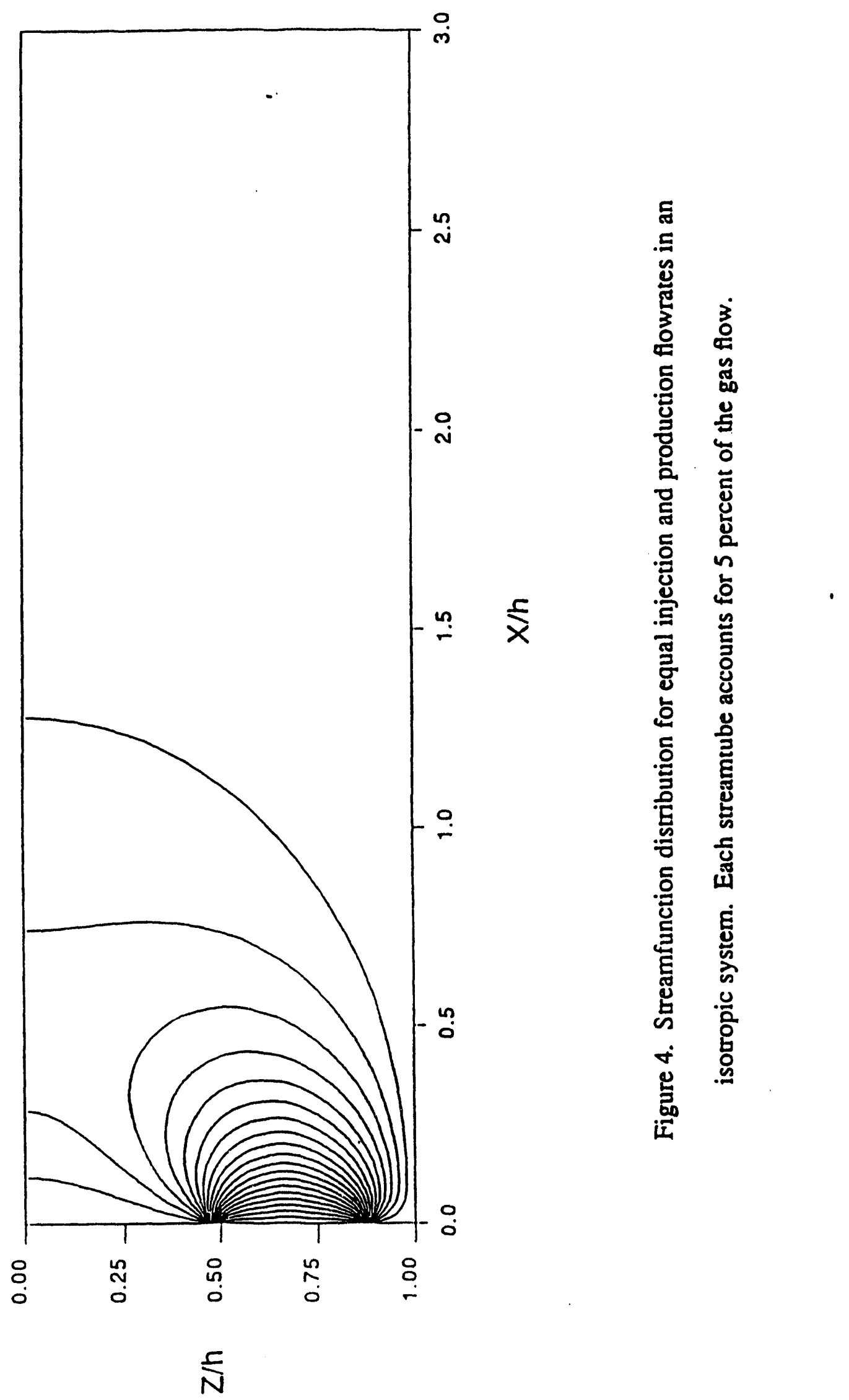




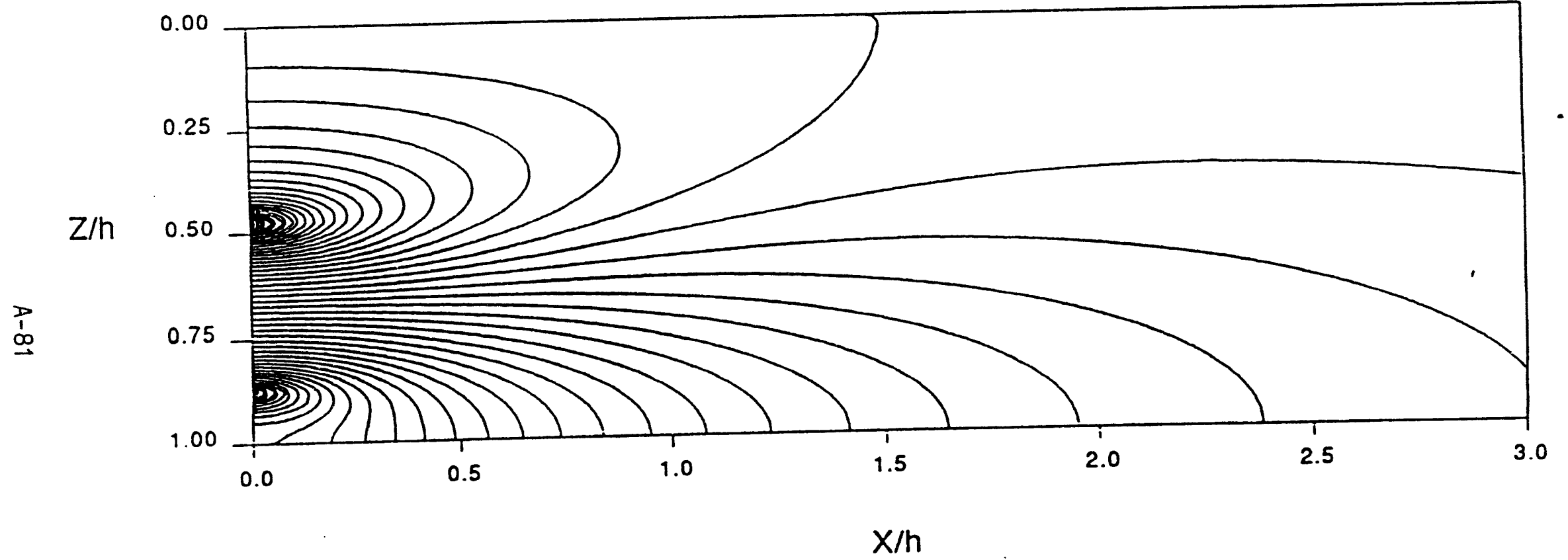

Figure 5. Gas pressure distribution for equal injection and production flowrates in an anisotropic system. The contour interval is 0.01 atmospheres. 


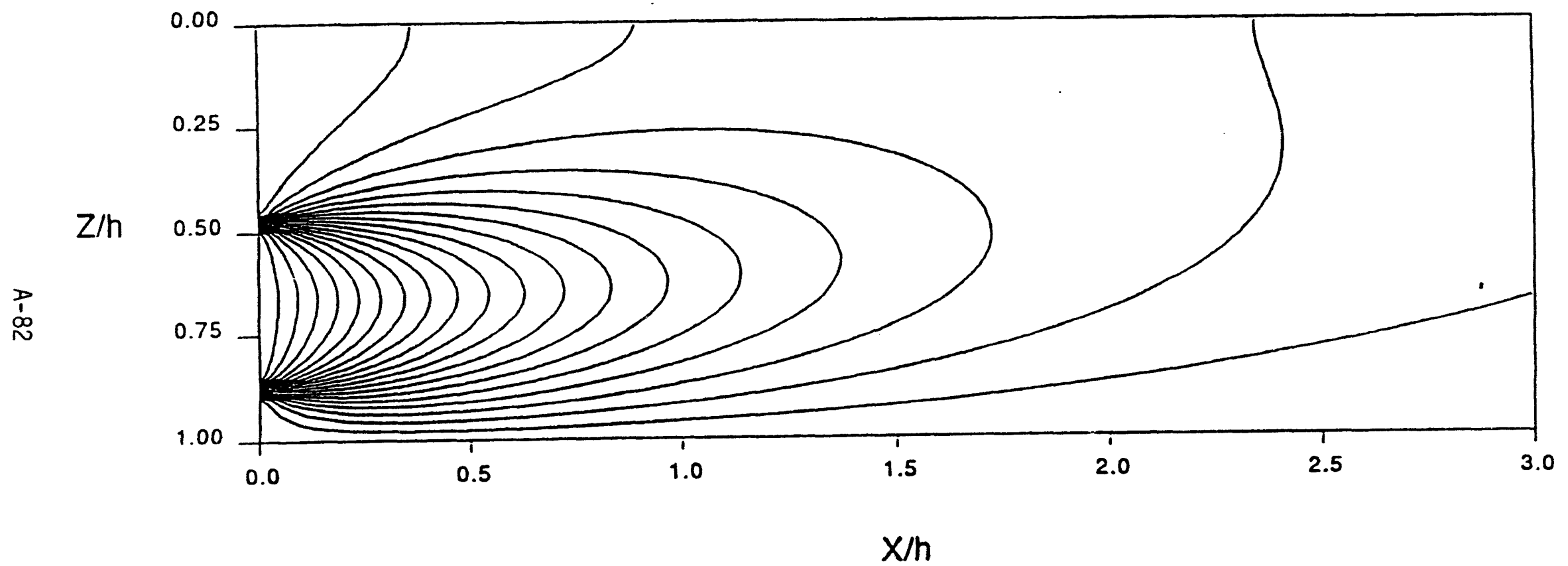

Figure 6. Streamfunction distribution for equal injection and production flowrates in an anisotropic system. Each streamtube accounts for 5 percent of the gas flow. 


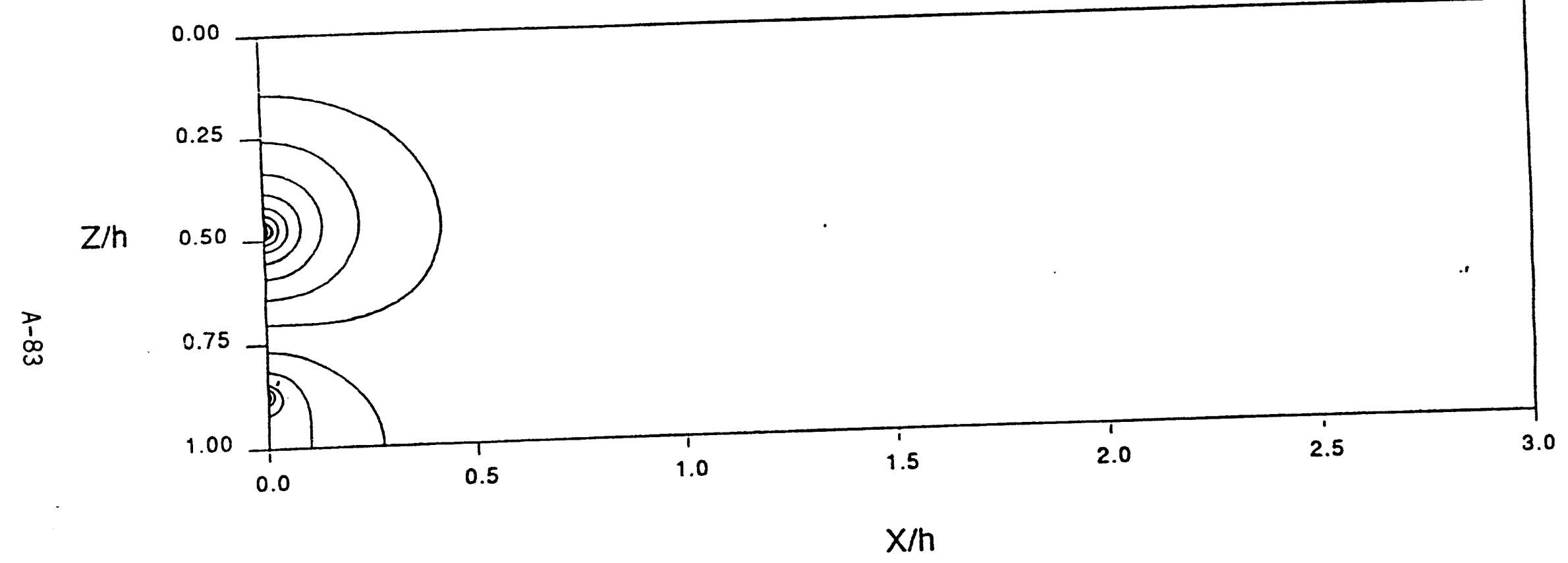

Figure 7. Gas pressure distribution for unequal injection and production flowrates in an isotropic system. The contour interval is 0.01 atmospheres. 


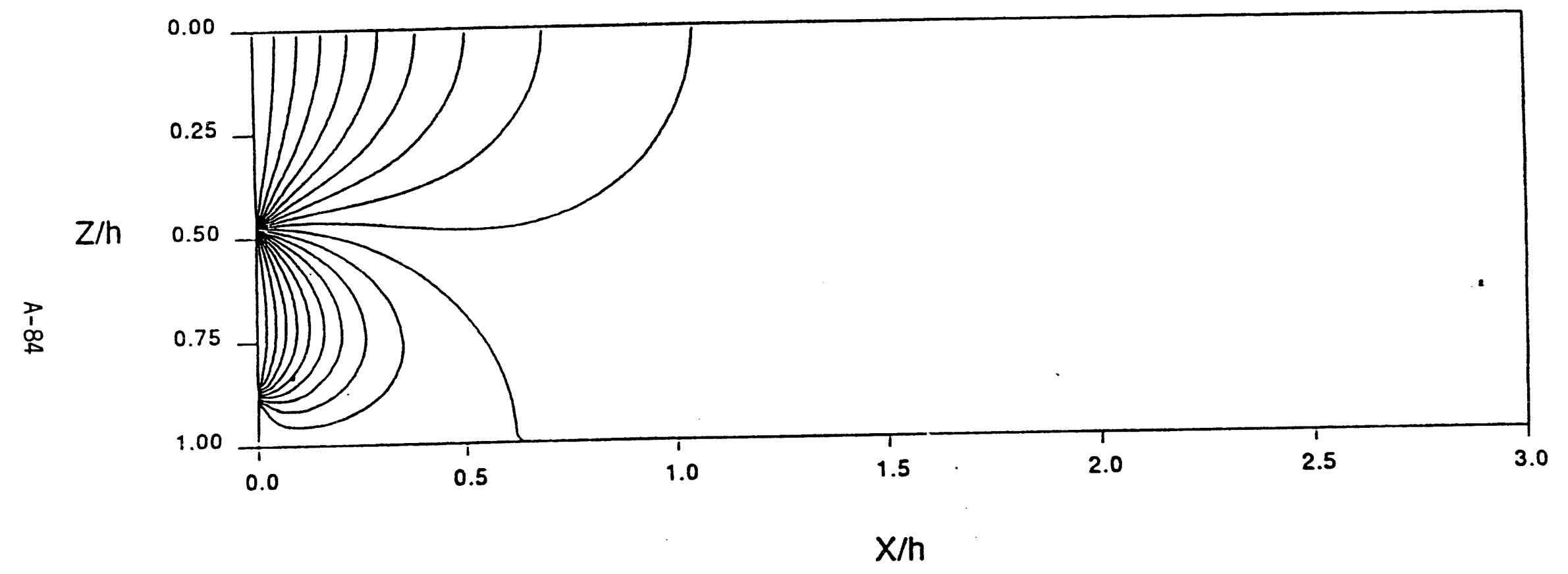

Figure 8. Streamfunction distribution for unequal injection and production flowrates in an isotropic system. Each streamtube accounts for 5 percent of the gas flow based on the upper well flowrate. 


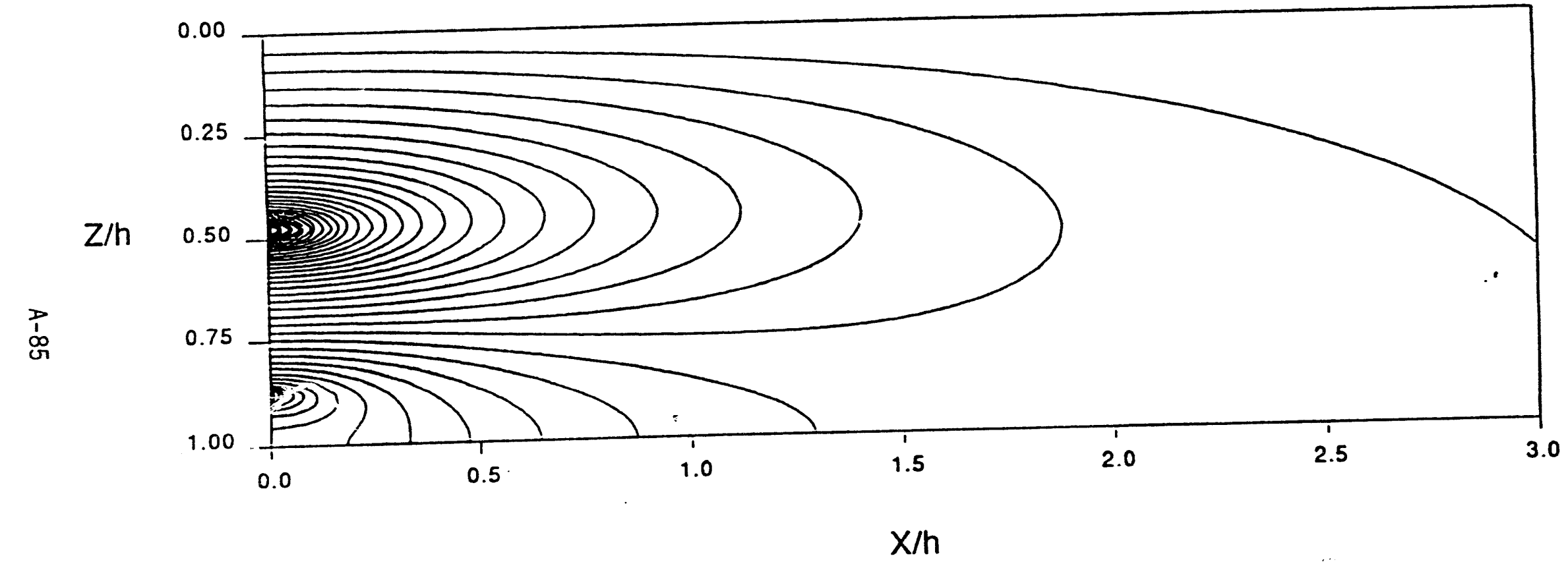

Figure 9. Gas pressure distribution for unequal injection and production flowrates in an anisotropic system. The contour interval is 0.01 armospheres. 


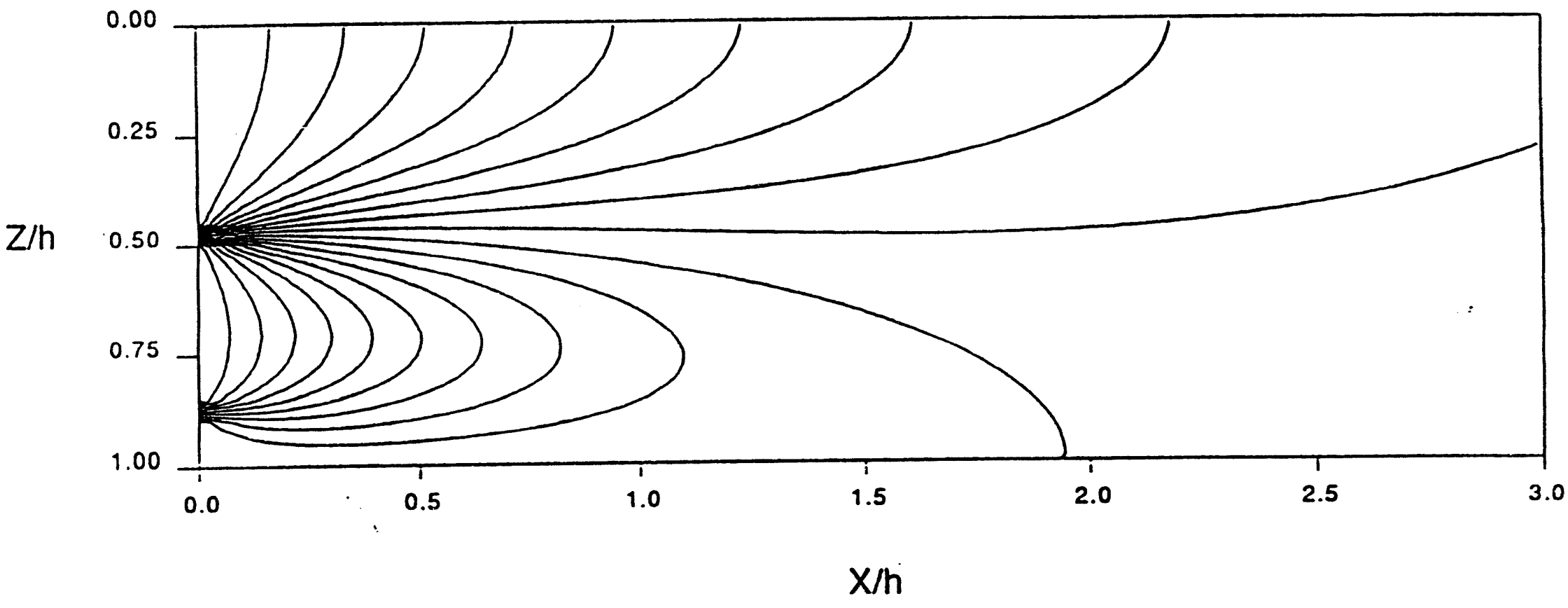

Figure 10. Streamfunction distribution for unequal injection and production flowrates in an anisotropic system. Each streamtube accounts for 5 percent of the gas flow based on the upper well flowrate. 


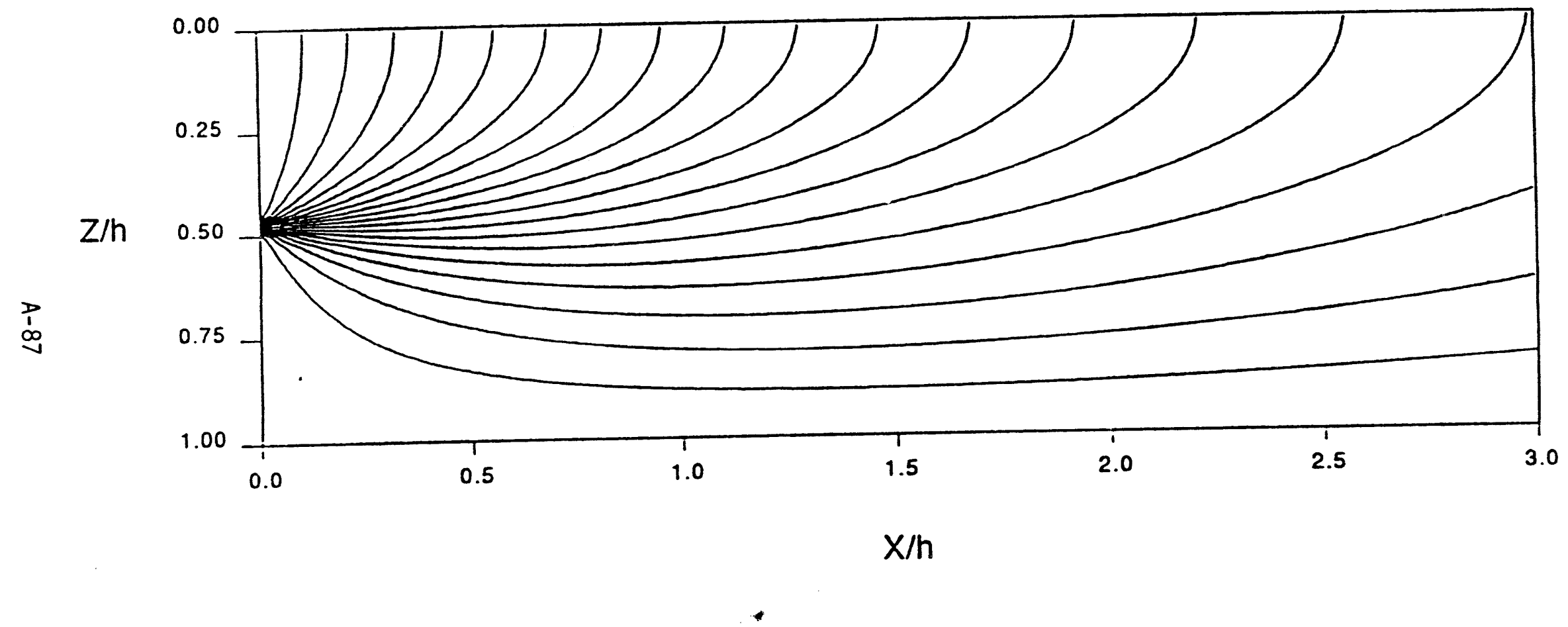

Figure 11. Streamfunction distribution for gas production from the upper well in in anisotropic system. Each streamtube accounts for 5 percent of the gas flow. 


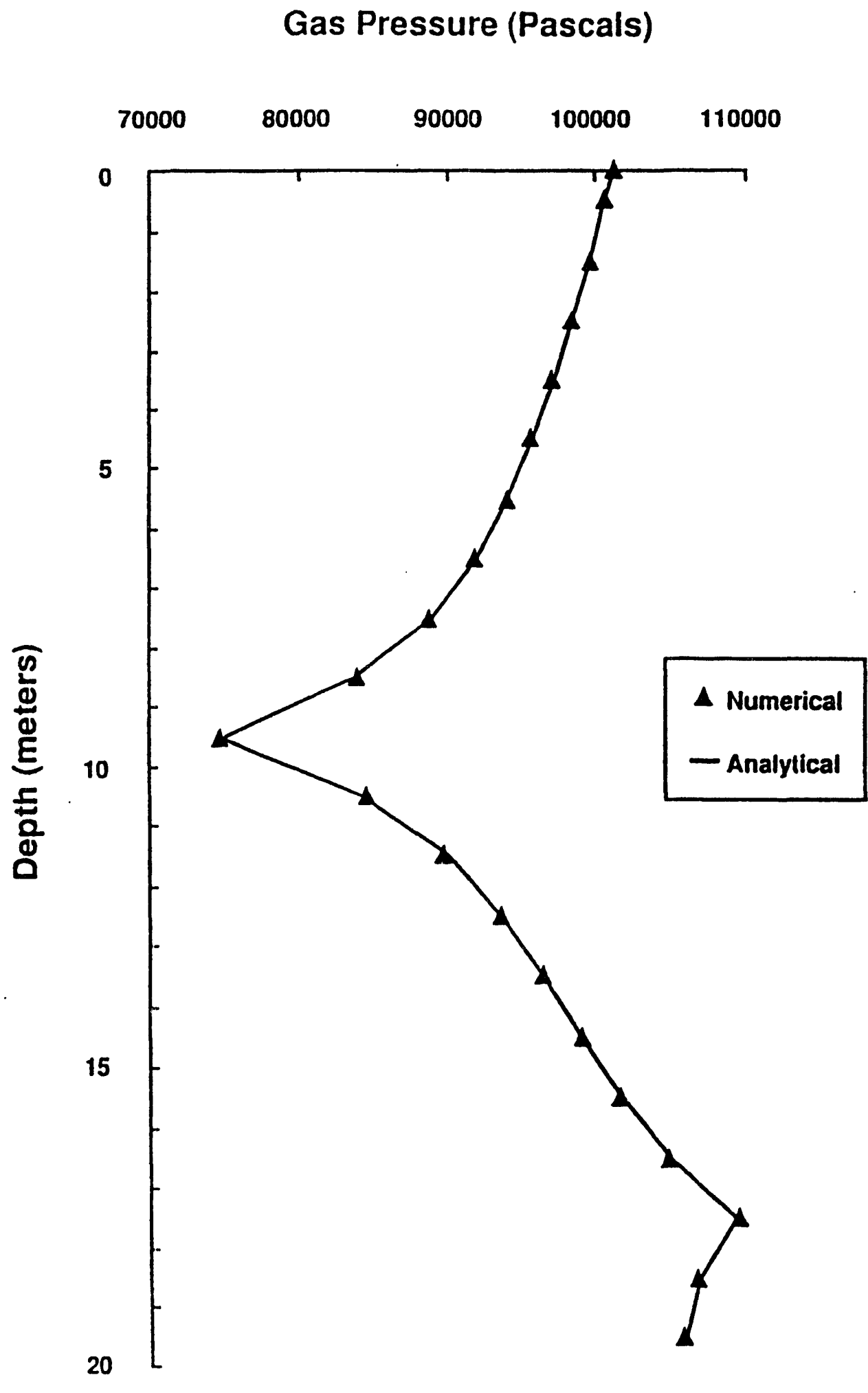

Figure 12. Comparison of analytically and numerically generated gas pressure distribution along a vertical line at $x=1.25 m$ for the case shown in Figures 9 and 10 . 


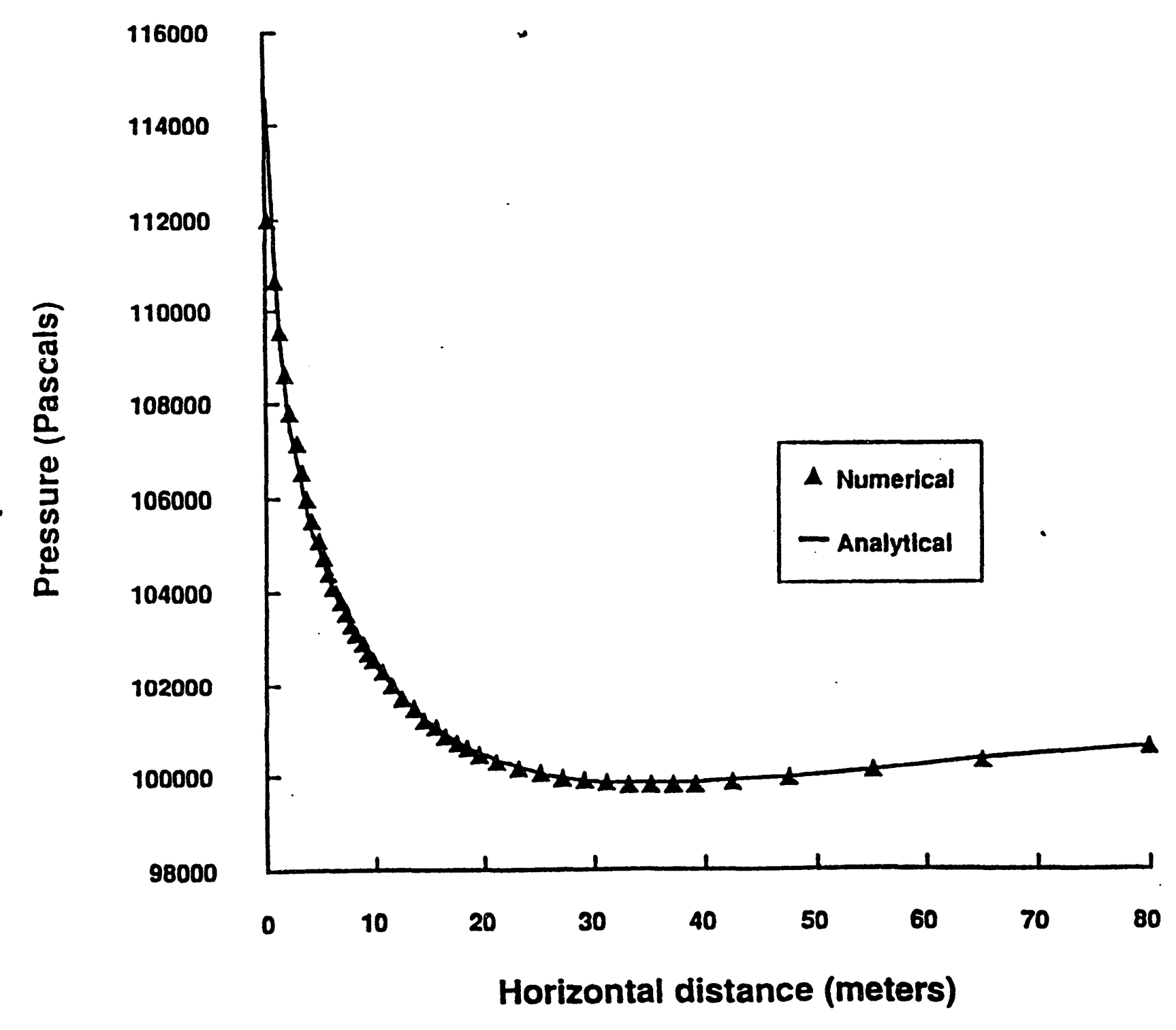

Figure 13. Comparison of analytically and numerically generated gas pressure distribution along a horizontal linc at $z=17.5 \mathrm{~m}$ for the case shown in Figures 9 and 10. 


\section{APPENDiX B: HeTEROgeneity PARAMETER MODELS}

In the first section of this Appendix, we present the derivation of a heterogeneity parameter model for waterflooding in considerable detail, since it seems conceptually simpler than starting immediately with the transport of volatile organic compounds. The extension to contaminant transport in a heterogeneous system can then be accomplished essentially by inspection after writing the equations for contaminant transport in an ideal system.

\section{WATERFLOODING}

In petroleum production, the primary recovery stage comprises the removal of oil, gas, and water from one or more wells (or producers) by reducing the bottom-hole pressure at the well-bore to a value below the pressure in the surrounding porous and permeable medium (the petroleum reservoir). In some reservoirs, the initial pressure, producing depth, and fluid density are such that the produced fluids will flow to the surface, but generally some form of artificial lift (e.g., a pump) is required before the end of primary production.

Many reservoirs have essentially closed outer boundaries, with little or no mass entering the system to replace fluids as they are produced. Hence, the reservoir pressure gradually declines in proportion to the cumulative fluid withdrawn (i.e., with increasing reservoir voidage), and they are known as depletion reservoirs. Economic primary production terminates when the pressure has declined to the point that oil and gas can no longer flow into the producers at rates sufficiently high to provide net revenues greater than the operating costs of the wells.

After a depletion reservoir has reached its economic limit on primary production, a significant percentage of the oil originally in place remains in the rock, along with gas, which exsolves as the reservoir pressure is reduced, and along with much of the water originally present. A portion of the remaining oil, kmown as the waterflood movable oil, can be recovered by water displacement; the remainder is the waterflood residual oil, trapped by capillary forces within the rock pores.

In the laboratory, when water is injected continuously into a rock core containing oil at a samuration higher than the waterflood residual value, some of the oil will be displaced to the outflow end of the core. The residual oil saturation value depends upon the initial oil saturation, the flow velocity, and various intrinsic properties of the rock/fluid system. The difference between the initial oil saturation, $S_{o i}$, and the waterflood residual oil saturation, $S_{\ldots m}$, is defined as the movable oil saturation. The residual oil after waterflooding represents the target for tertiary recovery processes, such as surfactant flooding.

In field applications of waterflooding, water is injected into the reservoir, either by converting some of the original producers to injectors, or by drilling new injectors, while continuing to withdraw fluids (usually by pumping) from the remaining producers. The injection of water not only restores the reservoir pressure, it also immiscibly displaces (or sweeps) oil from the injectors to the producers. Usually, the array of injectors and producers is designed to form a repeated pattern of basic symmetry elements, with the "fivespot" perhaps being the most common. Except near the edges of the reservoir, each injector in a five-spot pattern flood is surrounded by four producers, located at the comers of a square centered on the injector. A schematic illustration of a single element in a five-spot pattern is shown in Figure B.1.

The efficiency of a waterflood is most conveniently expressed as the fractional recovery of the total movable oil remaining in the reservoir after primary depletion. This allows the direct comparison of actual flood performance with an ideal system in which injected water volumetrically displaces, first, the gas left behind at the end of primary production, and then, the movable oil. No oil is produced until enough water has been injected to displace all the gas (the fill-up volume). Subsequently, oil alone is produced until water breaks through to the producing well, at which point the cumulative water injected is called the flood-out volume. 


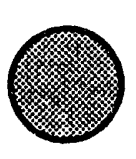

\section{$\not$}

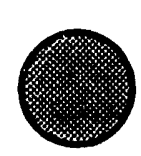

Figure B.1 Plan view of a single symmetry element of a five-spot waterflood pattern. The solid circles represent the producing wells, and the circle with the arrow through it represents the injection well. This basic element is repeated as needed to span the productive limits of the reservoir. A typical distance between producing wells in the US is about $400 \mathrm{~m}$.

\section{IDEAL Waterflood Performance}

This ideal, "piston-like" displacement process is shown graphically in Fiy. B.1 and can be described quantitatively through application of the following assumptions:

1. Before injection begins, the system is completely depleted by primary production, and there is no flow from the outflow end.

2. Fractional porosity $(\phi)$, along with initial oil, gas, and water saturations (expressed as fractions of the pore volume) $S_{o i}, S_{g i}$, and $S_{w i}$, respectively, are uniform, as is the intrinsic permeability, $k$.

3. Beginning at time $t=0$, water is injected at constant rate $i_{w}$ into one end of the system (the inflow boundary), successively displacing gas, oil, and water out the other end (the outflow boundary).

4. Flow between the inflow and outflow boundaries is perfectly linear. 
5. All displacements of one fluid by another are absolutely stable (i.e., piston-like), unaffected by gravity, capillarity, viscous fingering, dispersion, or any other process causing mixing or simultaneous flow of displacing and displaced fluids.

6. Injected water builds up an oil bank ahead of the injection front. Within the oil bank, only oil flows, at constant saturation (equal to $1-S_{w i}$ ); ahead of the bank, only gas flows (at saturation $S_{g i}$ ), and behind it, only water does (at saturation $1-S_{\text {orw }}$ ).

7. A residual oil saturation, $S_{\text {orw, }}$ is left behind after all the gas and movable oil have been displaced, and the remaining pore space is occupied by water at saturation $1-S_{\text {onw }}$.

Hence, at the outflow boundary (the producer), gas will be produced, with no liquid, until all the free gas has been displaced and the leading edge of the oil bank reaches the producer. Then, oil only will be produced until all the movable oil has been displaced (i.e., the trailing edge of the oil bank reaches the producer), and, finally, water only is produced until injection stops.

Consider a system of length $L$ and cross-sectional area $A$ perpendicular to the direction of flow, and let $V_{p}$, $V_{f u}$, and $V_{f o}$ represent, respectively, its total pore volume, fill-up volume, and flood-out volume:

$$
\begin{aligned}
& V_{p}=\phi \cdot A \cdot L \\
& V_{f i}=S_{g i} \cdot V_{p} \\
& V_{f o}=\left(S_{g i}+S_{o i}-S_{\text {onw }}\right) \cdot V_{p}
\end{aligned}
$$

Let $i_{w}$ be the rate of water injection, $q_{0}$ and $q_{w}$ be the oil and water volumetric production rates, respectively, and $W, Q_{0}$, and $Q_{w}$ be the cumulative water injected, oil produced, and water produced, respectively. Then, after injecting at constant rate for time $t$,

$$
\begin{aligned}
W & =i_{w} t & & \\
r_{0} & =0, & & \text { for } W<V_{f u} ; \\
& =W-V_{f u}, & & \text { for } V_{f u} \leq W<V_{f 0} ; \\
& =V_{f 0}-V_{f u}, & & \text { for } V_{f 0} \leq W \\
Q_{w} & =0, & & \text { for } W<V_{f 0} ; \\
& =W-V_{f 0}, & & \text { for } V_{f o} \leq W .
\end{aligned}
$$

Note that the limiting value of cumulative oil production, $Q_{0}$, is equal to the difference between the floodout volume and the fill-up volume, $V_{f o}-V_{f u}$, which is just the total movable oil in place at the start of waterflooding, $V_{\text {mo }}$.

The production rates, $q_{o}$ and $q_{w}$ can be obtained simply by differentiation of the equations for $Q_{o}$ and $Q_{w}$

$$
\begin{aligned}
q_{0} & =0, & & \text { for } W<V_{f u} ; \\
& =i_{w}, & & \text { for } V_{f u} \leq W<V_{f o} ; \\
& =0, & & \text { for } V_{f o} \leq W
\end{aligned}
$$




$$
\begin{aligned}
q_{w} & =0, & & \text { for } W<V_{f o} ; \\
& =i_{w}, & & \text { for } V_{f o} \leq W .
\end{aligned}
$$

It is convenient to normalize Eqs. (B.S) and (B.6), and the associated inequalities, by dividing both sides by $V_{m o}$, letting $\gamma=W N_{m o}$ be the normalized cumulative injection - i.e., the volume of water injected per unit volume of movable oil in place at the start of waterflooding:

$$
\begin{aligned}
Q_{o}^{*} & =0, & & \text { for } \gamma<\lambda ; \\
& =\gamma-\lambda, & & \text { for } \lambda \leq \gamma<1+\lambda, \\
& =1, & & \text { for } 1+\lambda \leq \gamma . \\
Q_{w}^{*} & =0, & & \text { for } \gamma<1+\lambda, \\
& =\gamma-1-\lambda, & & \text { for } 1+\lambda \leq \gamma .
\end{aligned}
$$

Here, $Q^{*}$ and $Q^{*}{ }_{w}$ are the normalized cumulative oil and water production, respectively. Note that the former quantity is just the oil recovery efficiency, expressed as a fraction of the movable oil in place at the start of waterflooding. The parameter $\lambda$ is the ratio of fill-up volume to movable oil volume. No oil is produced until the normalized cumulative injection is equal to $\lambda$. The recovery efficiency then increases linearly in direct proportion to normalized injection until all the oil has been produced (or injection stops). This was shown graphically in Fig. IV.2 and will not be repeated here.

Equations (B.T) and (B.8) are conveniently normalized by dividing the oil and water production rates by the water injection rate; the inequalities are normalized as before by dividing them through by the movable oil volume:

$$
\begin{array}{rlrl}
q_{0}^{*} & =0, & & \text { for } \gamma<\lambda ; \\
& =1, & & \text { for } \lambda \leq \gamma<1+\lambda, \\
& =0, & & \text { for } 1+\lambda \leq \gamma . \\
q_{w}^{*}=0, & & \text { for } \gamma<1+\lambda, \\
& =1, & & \text { for } 1+\lambda \leq \gamma .
\end{array}
$$

Figure B.2 (essentially the same as Fig. IV.1) shows a plot of the normalized oil production rate vs. normalized cumulative injection as given by Eq. (B.11). Plots for the other normalized variables defined in Eqs. (B.9), (B.10), and (B.12) are not shown, because they can easily be sketched by the reader.

\section{WATERFLOOd EFFICIENCY IN A HeTEROGENEOUS SYSTEM}

With the ideal linear system as a starting point, we can now consider the effect of the spatial variation in permeability. In order to simplify the analysis, the heterogeneous system is represented conceptually as a collection of independent linear elements connecting the inflow boundary (or boundaries) with the outflow 
boundary (or boundaries). The $j^{\text {th }}$ element has intrinsic permeability $k_{j}$ and area $a_{j}$ perpendicular to the direction of flow, and all elements are assumed to have the same length, porosity, and fluid saturations. The total area perpendicular to the direction of flow is $A$.

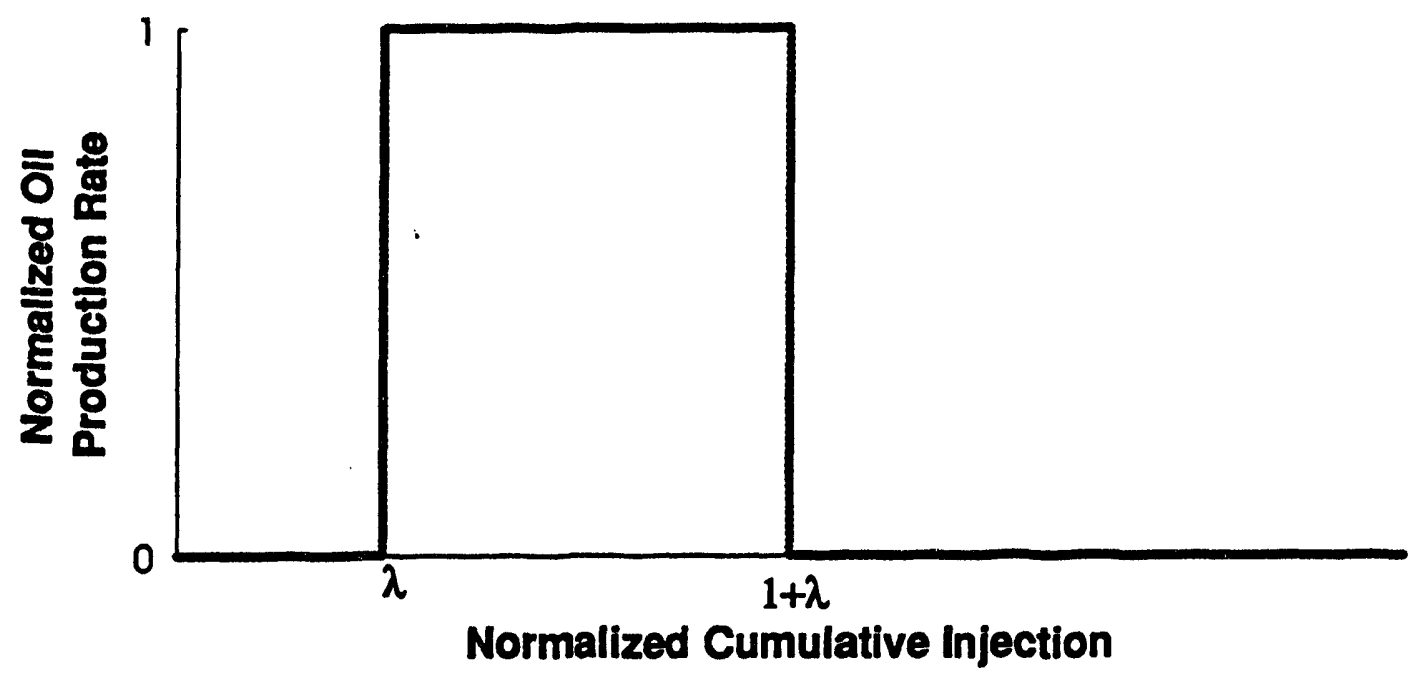

Figure B.2. Normalized oil production rate vs. normalized cumulative injection for an ideal homogeneous waterflood. The production rate is zero until all the gas remaining in the formation after primary depletion has been produced, then remains equal to the water injection rate until all the movable oil has been produced.

By ignoring changes in flow resistance (i.e., neglecting the saturation dependence of relative permeabilities) as the displacement fronts propagate through each element, and recognizing that the pressure drop between inflow and outflow must be the same for each element, we can write the cumulative injection into the $j^{\text {th }}$ layer, $W_{j}$, as:

$$
\begin{aligned}
W_{j} & =\frac{k_{j} \cdot a_{j}}{\sum_{j=1}^{N} k_{j} \cdot a_{j}} \cdot W \\
& =\frac{k_{j} \cdot a_{j}}{\bar{k} \cdot A} \cdot W
\end{aligned}
$$

Upon multiplying the numerator and denominator by $\phi \cdot L \cdot\left(S_{o i}-S_{o r w}\right)$ and rearranging the result, the normalized injection variable for element $j$ is found to be:

$$
\gamma_{j}=\gamma \cdot k_{j} / \bar{k}
$$


when the normalized cumulative injection into the entire system is equal to $\gamma$. Each element will obey Eqs. (B.7) and (B.8), with $\gamma_{j}$ in place of $\gamma$.

$$
\begin{array}{rlrl}
Q_{0}^{*}\left(k_{j}, \gamma\right) & =0, & & \text { for } k_{j}<\frac{\lambda \cdot \bar{k}}{\gamma} \equiv k_{1} ; \\
& =\frac{k_{j} \cdot \gamma}{\bar{k}}-\lambda, & & \text { for } k_{1} \leq k_{j}<\frac{(1+\lambda) \cdot \bar{k}}{\gamma} \equiv k_{2}, \\
& =1, & & \text { for } k_{2} \leq k_{j} . \\
Q_{w}^{*}\left(k_{j}, \gamma\right) & =0, & & \text { for } k_{j}<k_{2}, \\
& =\frac{k_{j} \cdot \gamma}{\bar{k}}-1-\lambda, & \text { for } k_{2} \leq k_{j} .
\end{array}
$$

Note that in Eqs. (B.15) and (B.16), the cumulative normalized oil and water production are written as

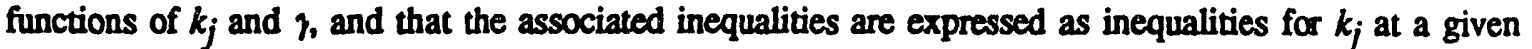
value of total dimensionless injection, $\gamma$. At a given value of $\eta$, all elements with $k$ less than $k_{1}$ will have produced no oil, those with $k$ between $k_{1}$ and $k_{2}$ will have produced only a fraction of their oil, and those with $k$ greater than $k_{2}$ will have produced all of their oil. Similarly, elements with $k$ less than $k_{2}$ will have produced no water, while those with $k$ greater than $k_{2}$ concinue to produce water so long as it is injected.

To obtain the total normalized production of oil and water, we must sum the contributions of all the elements, weighted by their volume fractions in the total system:

$$
\begin{aligned}
& Q_{0}^{*}(\gamma)=\sum_{j 3 k_{j}<k_{1}} 0 \cdot \frac{a_{j}}{A}+\sum_{j 3 k_{1} \leq k_{j}<k_{2}}\left[\frac{k_{j} \cdot \gamma}{\bar{k}}-\lambda\right] \cdot \frac{a_{j}}{A}+\sum_{j 3 k_{2} \leq k_{j}} 1 \cdot \frac{a_{j}}{A} \\
& Q_{w}^{*}(\gamma)=\sum_{j 3 k_{j}<k_{2}} 0 \cdot \frac{a_{j}}{A}+\sum_{j 3 k_{2} \leq k_{j}}\left[\frac{k_{j} \cdot \gamma}{\bar{k}}-1-\lambda\right] \cdot \frac{a_{j}}{A}
\end{aligned}
$$

With one additional assumption, the volumetric averages in Eqs. (B.17) and (B.18) can be replaced by averages over the permeability distribution of the system. We assume that this distribution is described by a probability density function $f(k)$, and that $f(k) d k$ represents the volume fraction with permeability between $k$ and $k+d k$, in accordance with the usual frequency interpretation of probability density functions. Hence, we can replace the fraction $a_{j} / A$ in the above equations by $f(k) d k$, and, in the limit as $d k$ approaches zero, the sums become integrals:

$$
Q_{o}^{*}(\gamma)=\frac{\gamma}{\bar{k}} \int_{k_{1}}^{k_{2}} k f(k) d k-\lambda \int_{k_{2}}^{k_{2}} f(k) d k+\int_{k_{2}}^{-} f(k) d k
$$




$$
Q_{w}^{*}(\gamma)=\frac{\gamma}{\bar{k}} \int_{k_{2}}^{\overline{0}} k f(k) d k-(1+\lambda) \int_{k_{2}}^{*} f(k) d k
$$

For many aquifers and petroleum reservoirs, the distribution of permeability values measured on cores is closely approximated by a log-normal distribution, with density function

$$
f(k)=\frac{\exp \left[-\frac{1}{2}\left(\frac{\ln k-\mu}{\sigma}\right)^{2}\right]}{k \sigma \sqrt{2 \pi}}
$$

The parameters $\mu$ and $\sigma$ are, respectively, the mean of the natural logarithm of $k$ and the square root of the variance of the natural logarithm of $k$. If we assume that the distribution of permeability is log-normal in Eqs (B.19) and (B.20), the integrations can be performed explicitly.

Note that there are only two types of integrals over the log-normal density function which are needed: one involving a partial average of $k$, and one involving an incomplete integral of the density function. By some straightforward but tedious manipulation involving changing the variable of integration and integrating by parts, it can be shown that, for arbitrary values of $a$ and $b$ :

$$
\begin{gathered}
\int_{\alpha \bar{k}}^{\Delta \bar{E}} f(k) d k=\Phi\left[\frac{\ln (b)+\frac{\sigma^{2}}{2}}{\sigma}\right]-\Phi\left[\frac{\ln (a)+\frac{\sigma^{2}}{2}}{\sigma}\right] \\
\int_{a \bar{k}}^{b \bar{E}} k f(k) d k=\bar{k}\left\{\Phi\left[\frac{\ln (b)-\frac{\sigma^{2}}{2}}{\sigma}\right]-\Phi\left[\frac{\ln (a)-\frac{\sigma^{2}}{2}}{\sigma}\right]\right\} \\
\Phi(z)=\frac{1}{\sqrt{2 \pi}} \int_{-}^{z} e^{-x^{2} / 2} \text { is the normal probability integral. }
\end{gathered}
$$


Dimensionless cumulative oil and water production, respectively, are then given by:

$$
\begin{aligned}
Q_{0}^{*} & =\gamma\left\{\Phi\left[\frac{\left.\ln \left(\frac{\gamma}{\lambda}\right)+\frac{\sigma^{2}}{2}\right]}{\sigma}\right]-\Phi\left[\frac{\ln \left(\frac{\gamma}{1+\lambda}\right)+\frac{\sigma^{2}}{2}}{\sigma}\right]\right\} \\
& +(1+\lambda) \Phi\left[\frac{\ln \left(\frac{\gamma}{1+\lambda}\right)-\frac{\sigma^{2}}{2}}{\sigma}\right]-\lambda \Phi\left[\frac{\ln \left(\frac{\gamma}{\lambda}\right)-\frac{\sigma^{2}}{2}}{\sigma}\right]
\end{aligned}
$$

$$
Q_{w}^{*}=\gamma \Phi\left[\frac{\ln \left(\frac{\gamma}{1+\lambda}\right)+\frac{\sigma^{2}}{2}}{\sigma}\right]-(1+\lambda) \Phi\left[\frac{\ln \left(\frac{\gamma}{1+\lambda}\right)-\frac{\sigma^{2}}{2}}{\sigma}\right]
$$

Normalized rates of production can be obtained by differentiating Eqs. (B.25) and (B.20) with respect to $\gamma$, and noting that

$$
\frac{q_{0}}{i_{w}}=\frac{d Q_{0}^{*}}{d \gamma}
$$

and

$$
\frac{q_{w}}{i_{w}}=\frac{d Q_{w}^{*}}{d \gamma}
$$

Again, the algebra is tedious but straightforward, and the resulting expressions are:

$$
\frac{q_{0}}{i_{w}}=\Phi\left[\frac{\ln \left(\frac{\gamma}{\lambda}\right)+\frac{\sigma^{2}}{2}}{\sigma}\right]-\Phi\left[\frac{\ln \left(\frac{\gamma}{1+\lambda}\right)+\frac{\sigma^{2}}{2}}{\sigma}\right]
$$




$$
\frac{q_{w}}{i_{w}}=\Phi\left[\frac{\ln \left(\frac{\gamma}{1+\lambda}\right)+\frac{\sigma^{2}}{2}}{\sigma}\right]
$$

In obtaining the final forms of Eqs. (B.25), (B.26), (B.29), and (B.30), the fact that $\Phi(-z)=1-\Phi(z)$ has been used.

Note that these expressions for dimensionless cumulative oil and water production and oil and water production rates reduce to those given previously for the ideal homogeneous case when $\sigma$ approaches 0 .

For $\sigma=0$, the normalized oil rate is a rectangular pulse, equal to zero until $\gamma=\lambda$, when it jumps to 1.0 , and remains there until $\gamma=1+\lambda$, when it returns to zero; this is precisely the behavior shown in Fig. B.2. The normalized water rate is a unit step function at $1+\lambda$ In fact, the oil pulse for the homogeneous case is just the difference between a step function for the leading edge of the oil bank and a step function for the trailing edge of the oil bank.

The median for each log-normal function in the heterogeneous system is the corresponding step-function argument multiplied by exp $\left(-\sigma^{2} / 2\right)$. Hence, as the heterogeneity parameter increases, the median breakthrough of each displacement front occurs at smaller and smaller values of cumulative injection.

The derivation of the waterflood model has been presented in some detail since it provides a comprehensible physical basis for the introduction of heterogeneity as a log-normal transformation on a step function representing the propagation of a displacement front in an ideal homogeneous system. Note that the heterogeneous system results are obtained by first developing equations describing the arrival of different fluid displacement fronts at the outflow boundary of an ideal linear system. The step-function arrival time (or, equivalently, the cumulative injection at which the arrival occurs) is then transformed into a cumulative log-normal distribution of arrival times by the derivation given above.

\section{VAPOR EXTRACTION}

\section{IDEAL SYSTEM}

To develop a model for vapor extraction, we again consider a linear element with area $A$ perpendicular to the direction of flow, and let $S_{v}$ be the total vapor content (air plus water vapor plus contaminant plus soil gas) per unit pore volume. The total bulk volume of sediment from which gas can flow to the outflow boundary (e.8., an extraction well) is assumed to be divided into two regions: an uncontaminated volume, $V_{u}$, nearest the outflow, and a contaminated volume, $V_{c}$, further from the outflow. This division is included to allow for a delay between the start of gas extraction and the beginning of contaminant removal - i.e., to allow for the case in which the extraction well is not located within the contaminant plume.

Within the contaminated volume, a volatile contaminant is assumed to be present in the vapor phase at concentration $C_{v o}$ (mass per unit volume of vapor phase), in equilibrium with contaminant at concentration $C_{l o}$ (mass per unit volume of aqueous phase) dissolved in a stationary aqueous phase held by capillary forces in the porous medium.

Water saturation is assumed equal to $1-S_{v}$ i in other words, there is no free non-aqueous phase liquid (NAPL) present. Solid-phase paritioning is also ignored (although it could be included, as in Falta et al., 1992). Vapor is extracted at a constant volumetric rate $q_{v}$ at the outflow well, and is supplied at some effective outer boundary either by injection, natural influx, or a combination of the two. Gas-phase compressibility is ignored. 
Consider a location $x$ at time $t$ between the inflow boundary at $x=0$ and the outflow boundary at $x=L$. At distances less than $x$, the medium is contaminated, and at greater distances the medium is uncontaminated. In other words, $\mathrm{x}$ is the position of the contaminant front (assumed sharp) at time $\mathrm{t}$. In time $\Delta t$, the carrier fluid (air) will move from $x$ to $x+\Delta x$, where

$$
\Delta x=\frac{q_{v}}{\phi S_{v} A} \Delta t
$$

Contaminant movement is retarded by partitioning into the stationary liquid phase. Let $f$ be the fraction of the length $\Delta x$ which becomes contaminated in time $\Delta t$ - i.e., the contaminant front will advance a distance $f \Delta x$.

A mass balance on the contaminant gives

$$
\begin{aligned}
C_{w} q_{v} \Delta t & =f \Delta x A \phi S_{v} C_{w}+f \Delta x A \phi\left(1-S_{v}\right) C_{v_{0}} \\
& =f\left(\frac{q_{v}}{\phi S_{v} A} \Delta t\right) A \phi S_{v} C_{w}+f\left(\frac{q_{v}}{\phi S_{v} A} \Delta t\right) A \phi\left(1-S_{v}\right) C_{b},
\end{aligned}
$$

where $\mathrm{Eq}$ (B.31) has been used to substitute for $\Delta x$.

After canceling common terms, the resulting expression can be solved for $f$ :

$$
f=\left(1+\left[\frac{1-S_{v}}{S v}\right] \frac{C_{b}}{C_{w}}\right)^{-1}
$$

The velocity of the contaminant front is

$$
v_{d}=f \frac{\Delta x}{\Delta t}=\frac{q_{v}}{\phi S_{v} A}\left(1+\left[\frac{1-S_{v}}{S v}\right] \frac{C_{b}}{C_{v 0}}\right)^{-1},
$$

where Eq. (B.31) was used to obtain the carrier fluid velocity. A similar analysis shows that the trailing edge of the contaminant moves with the same velocity. Hence, the contaminant moves as a rectangular pulse in this idealized model, exactly analogous to the movement of the oil bank in a waterflood. To complete the analogy, we need to determine the cumulative carrier fluid (air) throughput at the time the leading edge of the contaminant pulse reaches the outflow boundary, and the cumulative throughput when the trailing edge reaches the boundary.

Let the region from $x=0$ to $x=L_{c}$ be the originally contaminated portion, and the region from $L_{c}$ to $L$ be the uncontaminated portion of the system. Let $t_{f}$ be the time required for the front of the contaminant pulse to reach the outflow, and $t_{b}$ be the time for the back to reach the outflow. These are readily obtained by using Eq. (B.34) for the contaminant pulse velocity, and noting that the distances traveled are $L-L_{c}$ and $L$, respectively:

$$
t_{f}=\frac{L-L_{c}}{v_{c f}}=\frac{L_{c}}{v_{c f}}\left(L / L_{c}-1\right) \equiv \frac{\lambda L_{c}}{v_{c f}}
$$




$$
t_{b}=\frac{L}{v_{d}}=\frac{L_{c}}{v_{d}}\left(L / L_{c}\right) \equiv \frac{(1+\lambda) L_{c}}{v_{d}}
$$

where the parameter $\lambda$ has been introduced to maintain the analogy with the waterflood model. Note that it has the same effect in the ideal contaminant transport model that it does in the ideal waterflood model: viz., it introduces a delay in contaminant breakthrough at the outflow boundary of the system.

The form of Eqs. (B.35) and (B.36) suggests that an appropriate normalized cumulative throughput variable for vapor extraction can be defined as:

$$
\gamma_{v}=\frac{v_{q} t}{L_{c}}
$$

since contaminant extraction starts when $\gamma_{\psi}$ is equal to $\lambda$ (i.e., $t=t_{f}$ ) and ends when it is equal to $1+\lambda$ (i.e., $\left.t=t_{b}\right)$.

A more physically transparent definition for $\gamma_{v}$ is obtained by substituting the right hand side of Eq. (B.34) for $v_{c f}$

$$
\begin{aligned}
\frac{v_{\phi}}{L_{c}} & =\frac{q_{v}}{L_{c} \phi S_{v} A\left(1+\left[\frac{1-S_{v}}{S_{v}}\right] \frac{C_{b}}{C_{v}}\right)} \\
& =\frac{C_{v} q_{v}}{M_{\infty}}
\end{aligned}
$$

Note that the total initial mass of contaminant in the system, $M_{c o}$ is given by:

$$
\begin{aligned}
M_{\infty} & =L_{c} A\left[C_{w} \phi S_{v}+C_{b} \phi\left(1-S_{v}\right)\right] \\
& =L_{c} A \frac{C_{v o} \phi S_{v}}{f}
\end{aligned}
$$

Finally, the normalized throughput can be written as:

$$
\begin{aligned}
\gamma_{v} & =\left(\frac{C_{w_{0}}}{M_{c o}}\right)\left(q_{v} t\right) \\
& \equiv \frac{W_{v}}{\left(M_{c o} / C_{w}\right)}
\end{aligned}
$$


where the numerator is the cumulative volume of vapor (air, soil gas, water and contaminant vapor) removed at the outflow boundary, and the denominator is the amount that would have to be removed to extract all of the initial contaminant mass if $\lambda$ were equal to zero and the entire contaminant inventory could be extracted at constant concentration $C_{v o}$. This definition for normalized throughput hence has an analogous physical significance to the corresponding normalized quantity for a waterflood.

In complete analogy with Eqs. (B.9) and (B.11) for the normalized cumulative oil production and the normalized oil production rate for an ideal waterflood, we can write the following equations for normalized cumulative contaminant removal and normalized contaminant concentration in the produced gas for an ideal vacuum extraction process:

$$
\begin{array}{rlrl}
M / M_{\infty} & =0, & & \text { for } \gamma_{\nu}<\lambda ; \\
& =\gamma_{,}-\lambda, & & \text { for } \lambda \leq \gamma_{\nu}<1+\lambda, \\
& =1, & & \text { for } 1+\lambda \leq \gamma_{\nu} . \\
C_{v} / C_{w}=0, & & \text { for } \gamma_{\nu}<\lambda ; \\
& =1, & & \text { for } \lambda \leq \gamma_{\nu}<1+\lambda, \\
& =0, & & \text { for } 1+\lambda \leq \gamma_{\nu} .
\end{array}
$$

Here, $M$ is the cumulative total contaminant mass removed as a function of the normalized cumulative total volume of gas extracted, and $C_{v}$ is the corresponding contaminant concentration in the extracted gas. 


\section{HETEROGENEOUS SySTEM}

We can immediately write down the equations for a heterogeneous system comprised of infinitesimal linear elements with a log-normal distribution of permeability. The detailed steps are exactly the same as those used in deriving the waterflood equations. For the cumulative normalized contaminant mass extracted, we have

$$
\begin{aligned}
M / M_{\infty} & =\gamma_{\nu}\left\{\Phi\left[\frac{\ln \left(\frac{\gamma_{\nu}}{\lambda}\right)+\frac{\sigma^{2}}{2}}{\sigma}\right]-\Phi\left[\frac{\ln \left(\frac{\gamma_{\nu}}{1+\lambda}\right)+\frac{\sigma^{2}}{2}}{\sigma}\right]\right\} \\
& +(1+\lambda) \Phi\left[\frac{\ln \left(\frac{\gamma_{\nu}}{1+\lambda}\right)-\frac{\sigma^{2}}{2}}{\sigma}\right]-\lambda \Phi\left[\frac{\ln \left(\frac{\gamma_{\nu}}{\lambda}\right)-\frac{\sigma^{2}}{2}}{\sigma}\right]
\end{aligned}
$$

and for the normalized contaminant concentration in the extracted gas, we have

$$
C_{\nu} / C_{\infty}=\Phi\left[\frac{\ln \left(\frac{\gamma_{\nu}}{\lambda}\right)+\frac{\sigma^{2}}{2}}{\sigma}\right]-\Phi\left[\frac{\ln \left(\frac{\gamma_{\nu}}{1+\lambda}\right)+\frac{\sigma^{2}}{2}}{\sigma}\right] .
$$

Equations (B.43) and (B.44) reduce to Eqs. (B.41) and (B.42), respectively, as $\sigma$ approaches zero.

To illustrate the effect of the heterogeneity parameter, $\sigma$, on the cumulative contaminant extraction and the concentration of contaminant in the extracted gas, Fig. B.3 was prepared, assuming no delay between the start of vapor extraction and the arrival of contaminant at the extraction well (i.e., $\lambda=0$ ). Note that a $\log$-log scale is used in Fig. B.3. As pointed out in the discussion of the analogous waterflood model, the shapes of these curves should be invariant with respect to changes in scale factors when plotted with log$\log$ axes. Hence the parameters $\lambda$ and $\sigma$ can be determined by plotting field data on log-log paper, and comparing the resulting plots with a family of type curves for the normalized responses with systematically varying values of $\lambda$ and $\sigma$. The translations required along the horizontal and vertical axes to achieve a match between a type curve and the field plot can then be used to determine the parameters $C_{v o}$ and $M_{c o}$ by selecting a match point and reading off the corresponding values of the dimensionless quantities and field variables.

It is perhaps noteworthy that the general effects shown by these curves are consistent with observed performance in vapor extraction projects. In the ideal homogeneous linear system, contaminant is removed at a constant rate until it is all extracted, as shown in Fig. B. 3 for $\sigma=0$. Of course, this ideal behavior is never obtained in the field; in reality, the contaminant concentration in the extracted vapor 
decreases rapidly at first, but then continues to be produced at lower concentrations for a very long time. These lower concentrations remain significantly higher than regulatory standards until many times the ideal volume of gas has been extracted. This behavior is shown by the curves in Fig. B.3 for $\sigma \neq 0$.

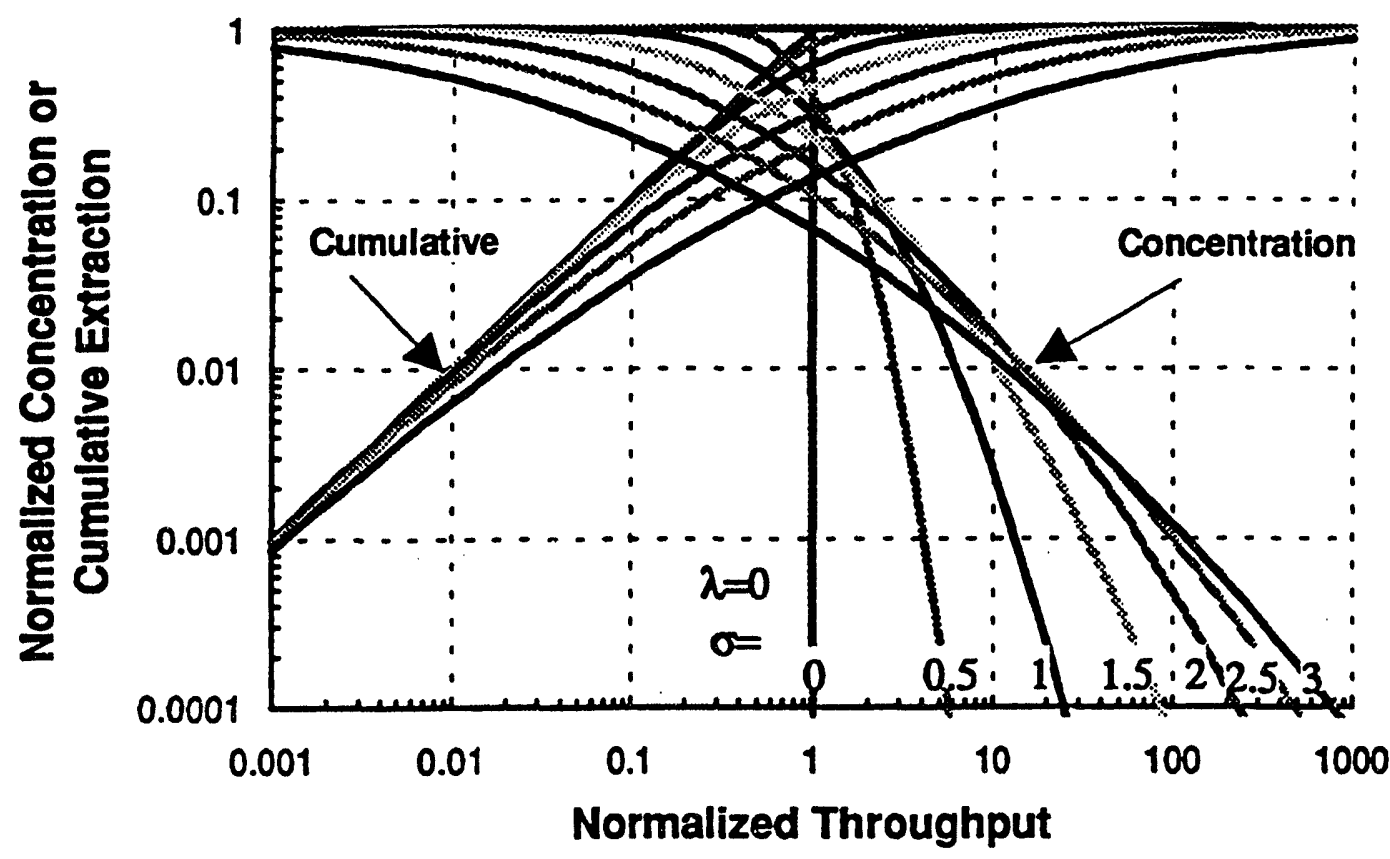

Figure B.1. Normalized contaminant concentration and normalized cumulative contaminant mass removed vs. normalized cumulative total vapor extracted (throughput) for $\lambda=0$, for values of $\sigma$ ranging from 0 to 3 . Note that there is no theoretical upper limit to the value of $\sigma$.

For the examples shown in Fig. B.3, note that attaining the three - to - four order of magnitude reduction in contaminant concentration typically needed to meet regulatory standards requires on the order of 10 times the ideal throughput volume for a system as heterogeneous as the Benton waterflood (i.e., $\sigma=0.8$ ). For even more heterogeneous systems, 100, 1000, or even larger multiples of the ideal throughput volume would be required to achieve regulatory compliance, depending upon the effective value of $\sigma$.

\section{PuMP AND TREAT}

\section{IDEAL SYSTEM}

For the vapor extraction process in an ideal system, we considered partitioning between a stationary aqueous phase and a mobile vapor phase, which serves as the carrier fluid. For a pump and treat process, the aqueous phase is the mobile carrier, and the solid grains in the sediment represent the stationary phase. We also noted that, since the dimensionless performance equations do not explicitly contain the partition coefficient, adsorption of contaminant on the solid could easily be accommodated in vadose zone vapor extraction processes. 
Provided that there is only a single mobile phase and that the concentrations in the stationary phases are proportional to the mobile phase concentration, the velocity of a contaminant front can generally be expressed as

$$
v_{\sigma^{\prime}}=\frac{v_{m}}{(1+K)}, \text { where } v_{m} \text { is the mobile phase particle velocity. }
$$

Here, $K$ is just the dimensionless ratio of total contaminant mass in the stationary phase(s) per unit bulk volume of sediment to the contaminant mass in the mobile phase per unit bulk volume of sediment. Note that, if all the contaminant is in the mobile phase, the contaminant front moves with the same velocity as the carrier fluid.

\section{HETEROGENEOUS SYSTEM}

As noted previously, dimensionless equations of the form of Eq. (B.43) for the cumulative contaminant mass extracted as a function of dimensionless carrier fluid throughput and Eq. (B.44) for contaminant concentration apply equally well to oil recovery, vapor extraction, and pump and treat with suitable definitions of dimensionless throughput variables. For contaminant extraction, the obvious choice of a dimensionless concentration variable is just the mass of contaminant per unit volume of carrier fluid in the extracted fluid, divided by an initial "average" concentration in the mobile phase within the contaminated zone. However, any consistent set of units can be used to fit the field data, with final conversion as required to calculate the contaminant mass inventory.

For a dimensionless throughput variable, we can use operating time divided by a characteristic time, $t_{0}$, or cumulative carrier fluid volume extracted divided by a characteristic volume, $Q_{0}$. That is, we can express concentration as a function of throughput as either

$$
c / c_{0}=\Phi\left[\frac{\ln \left(\frac{t / t_{0}}{\lambda}\right)+\frac{\sigma^{2}}{2}}{\sigma}\right]-\Phi\left[\frac{\ln \left(\frac{t / t_{0}}{1+\lambda}\right)+\frac{\sigma^{2}}{2}}{\sigma}\right] .
$$

or as

$$
c / c_{0}=\Phi\left[\frac{\ln \left(\frac{Q / Q_{0}}{\lambda}\right)+\frac{\sigma^{2}}{2}}{\sigma}\right]-\Phi\left[\frac{\ln \left(\frac{Q / Q_{0}}{1+\lambda}\right)+\frac{\sigma^{2}}{2}}{\sigma}\right] .
$$

In applications, Eq. (B.46) is the easiest to use if the carrier fluid extraction rate is reasonably constant, while Eq. (B.47) is better if the rate varies. 
In the former case, the initial contaminant mass inventory is calculated from

$$
M_{0}=c_{0} t_{0} q_{m} \text {. }
$$

where $q_{m}$ is : : a average carrier fluid extraction rate over the time period included in the analysis.

In the latter case, it is obtained from

$$
M_{0}=c_{0} Q_{0} \text {. }
$$

Note that only the product of the two scaling parameters is required to calculate the starting inventory of contaminant. Appendix C contains example calculations in fitting the Savannah River data. 


\section{Appendix C: Fitting Heterogeneity Parameter MOdels to Field Data.}

\section{BENTON WATERFLOOD}

There are several consequences of this simplified treatment that have practical significance. One is that for displacements in heterogeneous systems, the relevant operating variable is the logarithm of cumulative injection (or time, for more-or-less constant injection rates), which very vividly illustrates the rapidly diminishing retum for any recovery (or remediation) process involving fluid displacement. For the specific case of a waterflood, a plot of oil production rate vs. time on a logarithmic scale should result in a symmetric Gaussian curve shape, as shown in Fig. C.1 for an Illinois Basin waterflood (Benton Field) from 1947 until 1972.

\section{Benton Waterflood}

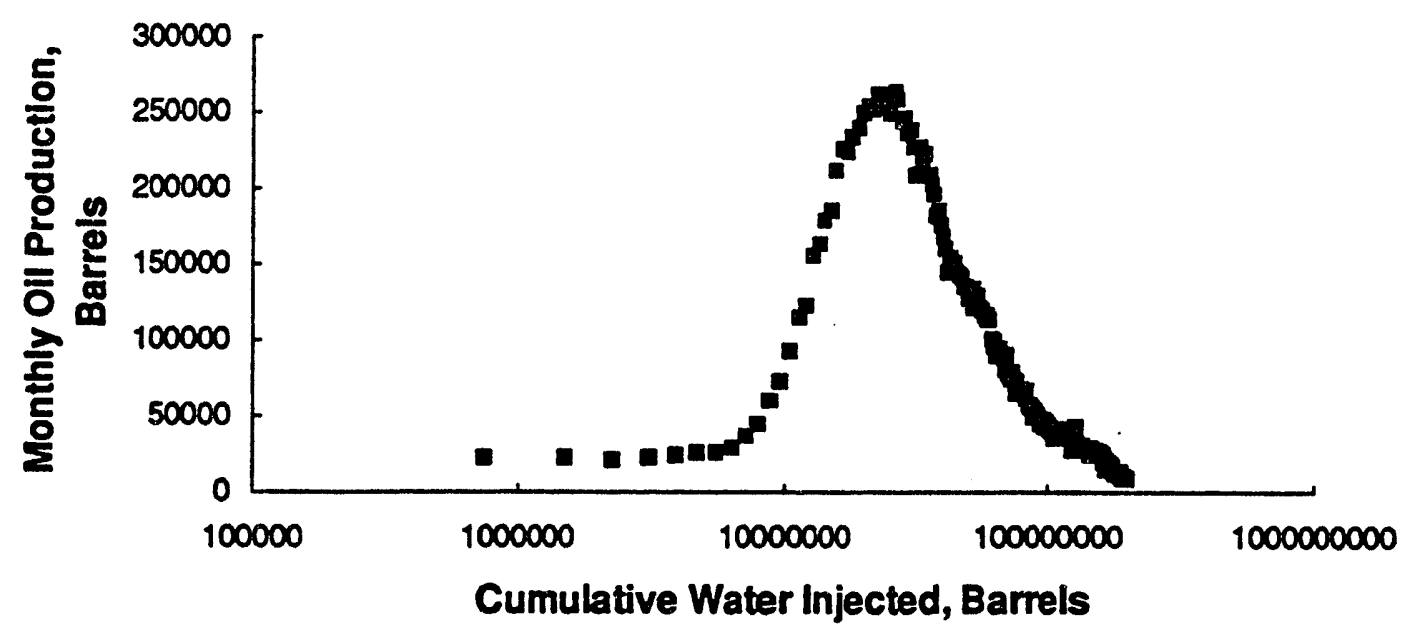

\section{Figure C.1. Monthly Oil Production versus Cumulative Water Injected for the Benton Waterflood, Benton, Illinois.}

Perhaps even more significant is that the size of the system does not appear explicitly in the normalized response equations. Changing the total movable oil in place merely translates the model production response curve along the horizontal (logarithmic) axis. If the vertical axis is made logarithmic as well, changing scales translates the curve horizontally and vertically without changing its shape. This implies that, provided there is some statistical regularity within a single reservoir being waterflooded, the shape of production curves for individual wells or groups of wells should be similar to the curve for the entire reservoir when plotted on $\log -\log$ axes. This conclusion was shown to be correct, for the Benton waterflood, in Chesnut et al. (1978).

Finally, there is a self-contained test of the theory. Note that the normalized water production rate is given by a single log-normal probability integral (Eq. B.30) and that the sum of oil and water (i.e., total liquid) production rates is given by a different log-normal integral with the same $\sigma$ but a different median. Plots of normalized water and total liquid production rates vs. $\log$ of time on probability paper should result in parallel straight lines. 
Figures C. 2 and C. 3 show, respectively, probability plots of total liquid production rate vs. $\log \mathrm{W}$ and water production rate vs. $\log \mathrm{W}$. In addition to the field data, these plots also show straight lines obtained from a least-squares fit to the data. The slopes of these lines give directly the values of $\sigma$ from the total liquid response $(0.813)$ and from the water production response $(0.804)$. If the model is a good approximation to the displacement of fluids in heterogeneous media, these $\sigma$ values should be identical. The agreement is quite good, especially in view of the gross simplifying assumptions made in deriving the model equations, and suggests that the shape of breakthrough curves for field-scale transport processes may be dominated by spatial heterogeneities rather than by classical dispersion theory, which predicts a Gaussian breakthrough curve.

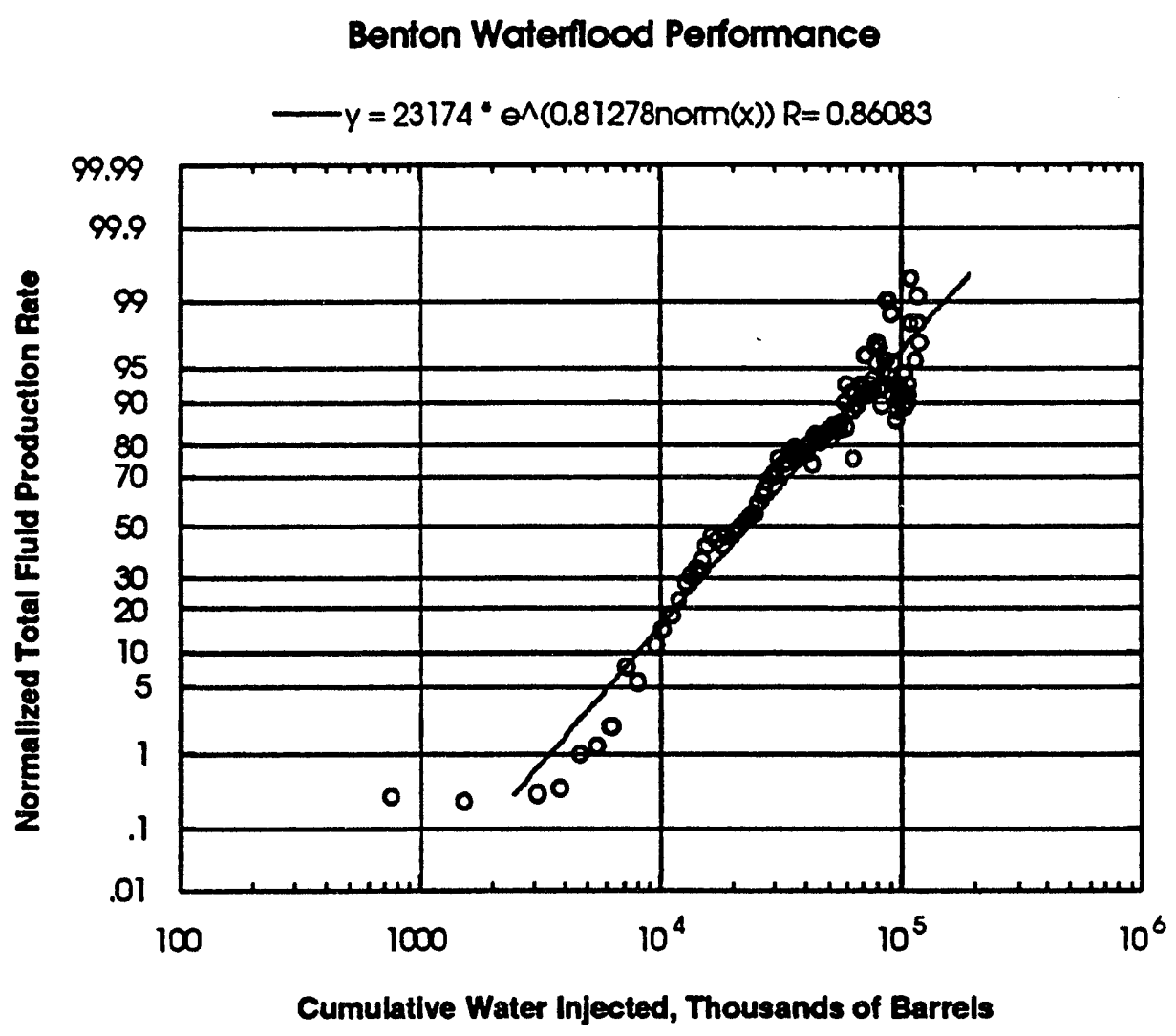

Figure C.2. Log-normal probability plot of normalized total fluid production rate vs. cumulative water injected, for field data (circles) and a linear leastsquares fit to the data (dashed line). 


\section{- Benton Waterflood Performance}

$-y=35072 \cdot \theta \wedge(0.80422 n o m(x)) R=0.96233$

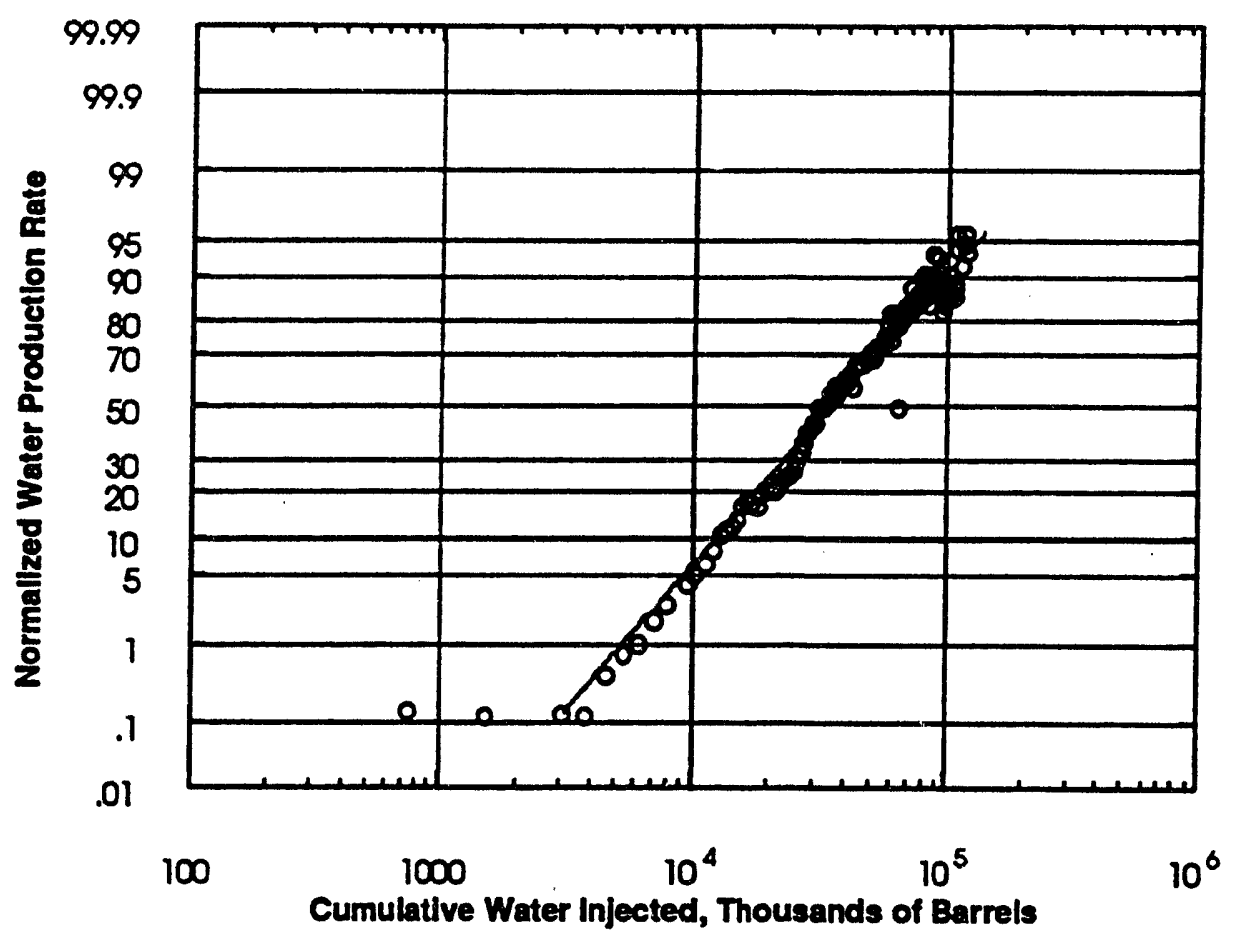

Figure C.3. Log-normal probability plot of normalized water production rate vs. cumulative water injected, for field data (circles) and a linear leastsquares fit to the data (dashed line)

\section{SAVANNAH RIVER IN-SitU AIR STRIPPING DATA}

\section{Air Injection History and Operating Time}

The air injection history was obtained from copies of field log book pages reproduced in Looney et. al. (1991b - draft copy):

Table C.1. Summary of Air Injection History

\begin{tabular}{|l|l|}
\hline Time interval, hr. & Nominal Injection Rate, SCFM \\
\hline $0-342.5$ & 0 \\
$342.5-655$ & 65 \\
$655-1511.6$ & 170 \\
$1511.6-2261.6$ & 270 \\
$>2261.6$ & 0 \\
\hline
\end{tabular}


No $\log$ book data were available to us after $11 / 8 / 90$. We used the first clock reading of the unit hours clock on $11 / 16 / 90,2611.5$ hours, from the FTESTVEXIS spreadsheet sent to us by B. B. Looney on 5/11/92, as the time that air injection stopped. This was used to calculate a value of 2261.6 hours for the operating time at the termination of air injection. Anomalously low readings for both TCE and PCE from 2172.5 to 2173.2 operating hours were not used in fitting the data. These are the concentrations resulting in the sharp downward spikes shown in Fig. C.4.

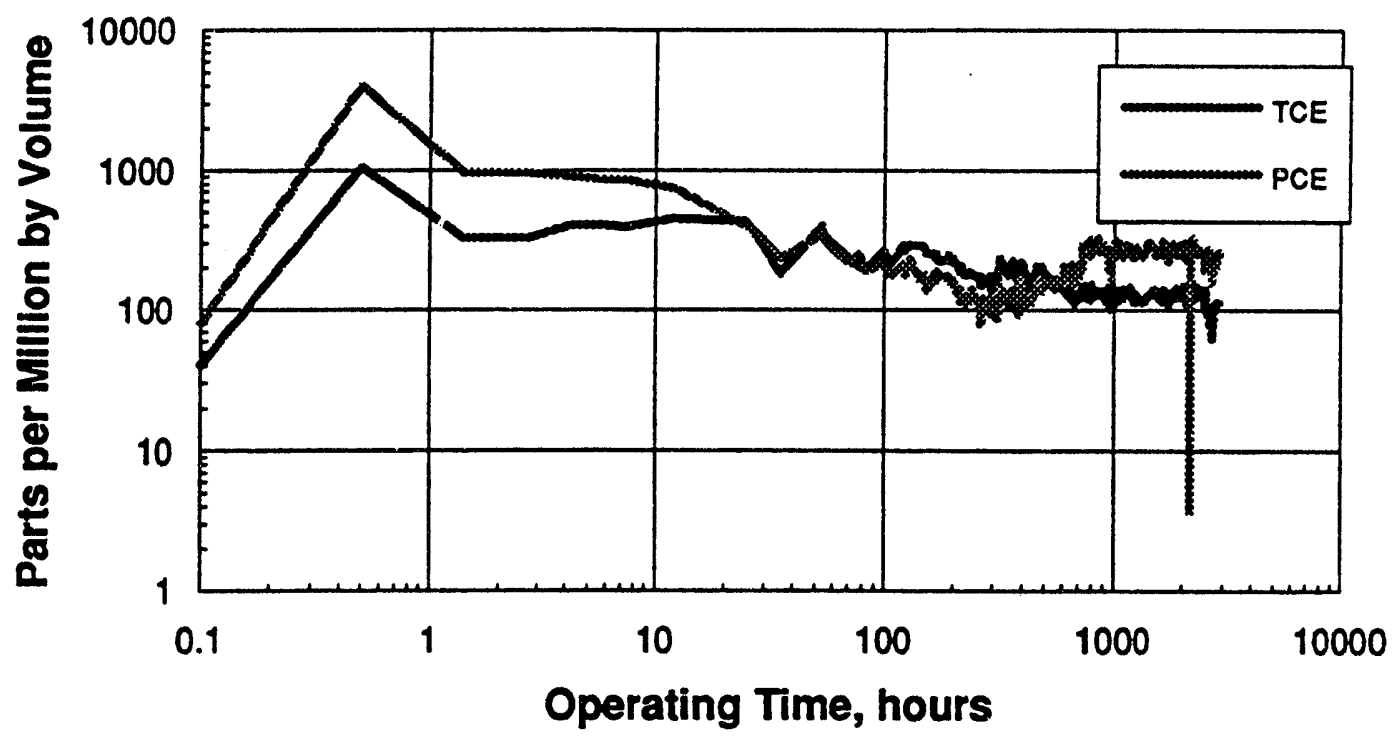

Figure C.4. Contaminant concentrations (ppm by volume) measured in gases extracted from horizontal well AMH-2 during the Savannah River Integrated Demonstration of In-Situ Air Stripping.

A portion of the PCE_TCEXIS spreadsheet is shown in Fig. C.5. The first, third, and fourth columns were extracted from FTESTVE.XIS received from B. Looney on 5/11/92. Column 2 gives the hours of

\begin{tabular}{|c|c|c|c|c|c|c|c|c|c|c|c|c|c|}
\hline \multicolumn{4}{|c|}{ 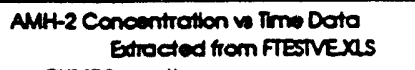 } & & & & $\boldsymbol{\lambda}$ & $\begin{array}{r}\text { TCE } \\
1.00 \leqslant 01\end{array}$ & $\begin{array}{r}\text { PCE } \\
1.00 E-\infty\end{array}$ & & & $\begin{array}{r}14 \\
\text { TCE } \\
8\end{array}$ & $\begin{array}{r}13 \\
\text { PCE } \\
\text { OS }\end{array}$ \\
\hline \multicolumn{4}{|c|}{$\begin{array}{l}\text { PUMPPDerdith } \\
\text { nMEE time TCE PCE }\end{array}$} & & & & $\hat{\sigma}$ & 25 & 25 & & & as & 1.1 \\
\hline \multirow{4}{*}{ ARS) (ors) } & & PPM) & (PPM) & $x$ & 500 & & $\infty$ & 1000.0 & 15000 & & & 1700 & \\
\hline & & & & & & & Cl & 8000 & 1500 & & & & 5000 \\
\hline & & & & & & & $\mathbf{M W}$ & 1314 & 1658 & & & 1314 & 1058 \\
\hline & & & & & & & $M \infty$ & 101863 & 36150 & & & 10020 & 562464 \\
\hline 1 & 2 & $\mathbf{s}$ & 4 & 5 & 6 & 7 & 8 & 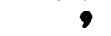 & 10 & 11 & 12 & & \\
\hline 16042 & & & & Hown 1 & CELOBS & PCECos & rioction 1 & reEcNe R & CECNC D & a_ree 0 & DAPCE & & \\
\hline 10043 & 0.1 & 39.6 & 78.7 & 0.1 & 398 & 78.7 & 0 xcm & 60.7 & 79.5 & -28.0 & -7008 & & \\
\hline 10047 & 0.5 & 10020 & 3969.5 & 0.5 & 10520 & 3969.5 & & 176.7 & 91.5 & 875.3 & 3000.0 & & \\
\hline 16056 & 14 & 328.7 & 9566 & IA & 328.7 & 9566 & & 2642 & 9342 & 64.5 & 225 & & \\
\hline 16069 & 27 & 327.7 & 955.1 & 27 & 327.7 & 955.1 & & 3145 & 858.3 & 132 & 969 & & \\
\hline 16004 & 42 & $\$ 01.0$ & 901.4 & 42 & $\$ 01.0$ & 9014 & & 341.1 & 7872 & 59.9 & 1142 & & \\
\hline 1610.1 & 5.9 & $\Delta 023$ & 8525 & 5.9 & 4023 & 8525 & & 35600 & 724.7 & 46.3 & 127.8 & & \\
\hline 16116 & 74 & 3902 & 8255 & 74 & 3902 & 852.5 & & 3629 & 6004 & 274 & 1721 & & \\
\hline 16161 & 11.9 & 446.1 & 7460 & 11.9 & 446.1 & 746.0 & & 360.5 & 503.9 & 77.7 & 1620 & & \\
\hline 1628.7 & 24.5 & 431.8 & $\Delta 025$ & 245 & 431.8 & $\triangle 005$ & & 353.7 & 4362 & 78.1 & 35.7 & & \\
\hline 16340 & 29.8 & 208.0 & 3260 & 29.8 & 284.0 & 325.0 & & 3452 & 400.7 & 57.2 & .74 .8 & & \\
\hline 1639.4 & 35.2 & 178.4 & 229.7 & 352 & 170.4 & 229.7 & & 3366 & 370.0 & -158.3 & -140.3 & & \\
\hline 1654.3 & 50.1 & 352.0 & 3282 & 50.1 & 3520 & 328.2 & & 3148 & 300.6 & 372 & 19.6 & & \\
\hline 1657.8 & 536 & 3952 & 391.6 & 53.6 & 395.2 & 391.6 & & 310.1 & 297.5 & 85.1 & 940 & & \\
\hline 1650.3 & 54.1 & 3458 & 329.4 & 54.1 & 345.8 & 329.4 & & 309.5 & 296.0 & 36.4 & 334 & & \\
\hline 10528 & 58.6 & 3026 & 2821 & 58.6 & 3026 & 282.1 & & 303.8 & 283.2 & -12 & -12 & & \\
\hline 1676.1 & 71.9 & 230.5 & 211.8 & 71.9 & 230.5 & 211.8 & & 2884 & 252.0 & 57.9 & 40.2 & & \\
\hline 1676.6 & 724 & 230.5 & 219.5 & 724 & 230.5 & 219.5 & $\therefore$ & 287.8 & 251.0 & 57.3 & -31.5 & & \\
\hline 1681.5 & 77.3 & 205.4 & 248.9 & 77.3 & 205.4 & 248.9 & & 282.7 & 241.5 & -772 & 7.4 & & \\
\hline 1687.7 & 83.5 & 180.8 & 190.3 & 83.5 & 188.8 & 190.3 & & 276.5 & 2306 & -87.7 & -40.2 & & \\
\hline 1099.9 & 95.7 & 248.3 & 211.9 & 95.7 & 248.3 & 211.9 & & 265.3 & 212.0 & -17.0 & -02 & & \\
\hline 1700.3 & 96.1 & 2412 & 211.9 & 9.1 & 2412 & 211.9 & & 265.0 & 211.5 & -23.8 & 0.4 & & \\
\hline Fimen & & $\begin{array}{l}\mathbf{P a} \\
\text { IS }\end{array}$ & dr & $\begin{array}{c}\mathrm{T} \\
\mathrm{e} \text { he }\end{array}$ &.$X I$ & eit & ads & $\begin{array}{l}\text { eet } \mathbf{u} \\
\text { neter }\end{array}$ & $\begin{array}{l}\mathrm{d} \text { to fi } \\
\text { nodel. }\end{array}$ & $\mathbf{t} \mathbf{S a}$ & anna & River & \\
\hline
\end{tabular}


operating time calculated from the clock readings in Col. 1. Columns 5, 6, and 7 were copied from Cols. 2, 3. and 4 (values only) with gaps due to down time removed. Values from 2172.2 to 2173.2 operating hours were deleted, as discussed above.

In fitting the data, the first step was to match the initial operating period, before air injection started. This was done with a Mathcad worksheet, FITCLEAN.MCD, by trial and error. Columns 5,6, and 7 were exported from PCE_TCE.XIS as a text file to be read by FITCLEAN.MCD as a 3-row by N-Column matrix, where $\mathrm{N}$ is the number of time points included. An example trial for fitting PCE concentration vs. time data is shown in Fig. C.6.

MCAD worksheet FITCLEAN modified from LOGCLEAN to visually fit field data for contaminant concentration vs. time. $\Gamma$ is the dimensionless cumulative volume of carrier fluid (air $\alpha$ water) removed from the system at an outflow boundary (one or more wells), $\lambda$ is a delay parameter to account for delayed breakthrough of contaminam, and $\sigma$ is a macroscopic heterogeneity parametes.

DA. Chesnut, May 20, 1992

Functions:

$g(\Gamma, \lambda, \sigma):=\cos \left[\frac{\left.\ln \left(\frac{\Gamma}{\lambda}\right)+\frac{\sigma^{2}}{2}\right]}{\sigma}\right]-\cos \left[\frac{\ln \left(\frac{\Gamma}{\lambda+1}\right)+\frac{\sigma^{2}}{2}}{\sigma}\right]$
$h(\Gamma, \lambda, \sigma):=-\lambda \cdot \cos \sigma\left[\frac{\ln \left(\frac{\Gamma}{\lambda}\right)-\frac{\sigma^{2}}{2}}{\sigma}\right]+(1+\lambda) \cdot \cos \sigma m\left[\frac{\ln \left(\frac{\Gamma}{\lambda+1}\right)-\frac{\sigma^{2}}{2}}{\sigma}\right]$

Sot up array of argument values

$\Gamma_{\max }:=1000 \quad \Gamma_{1}:=0.001 \quad \delta:=0.05 \quad \varepsilon:=10^{-15}$

i : $=2 . .121$

Trial values of shape parameters: $\quad \lambda:=.01 \quad \sigma:=2.5$

$r_{i}:=\Gamma_{i-1} \cdot 10^{8}$

Calculate dimensionless concentration and cumulative mass removed

$i:=1 . .121 \quad c_{i}:=g\left(\Gamma_{i}, \lambda, \sigma\right)+\varepsilon \quad m_{i}:=\Gamma_{i} \cdot c_{i}+h\left(\Gamma_{i}, \lambda, \sigma\right)$

Read matrix of field data: con :=READPRN (tce_pce )

$j:=3$.. rows (con) Set range of index for plotting

$\infty:=1500 \quad$ cl $:=150 \quad$ Trial values for scale parameters

PCE concentration

versus time

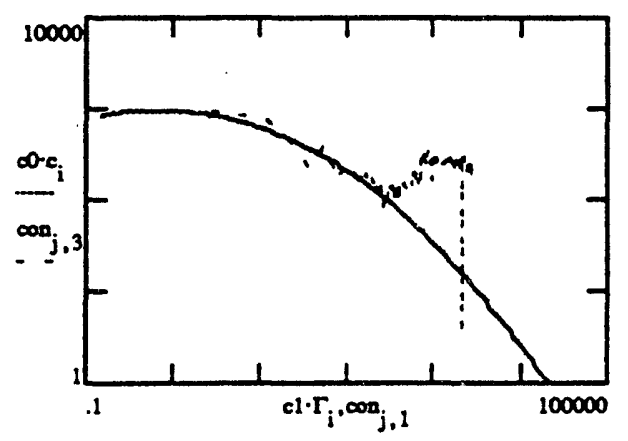

Figure C.6. Mathcad worksheet for fitting early-time data (extraction only). 
The final values for the parameters $\lambda, \sigma, c_{0}$ and $t_{0}$ ( $t_{0}$ is labeled as $c_{1}$ in PCE_TCE.XIS and FITCLEAN.MCD) are given above Cols. 9 and 10 of PCE_TCE.XIS for TCE and PCE, respectively, and were shown previously in Table IV.1 in the columns for "VE." Conversions from field units to mass per unit volume, and the calculation of total contaminant inventories, were explained in Section IV.

Columns 9 and 10 in PCE_TCEXIS are calculated values of TCE and PCE concentrations in ppm by volume, using the parameters determined by the trial and error fit with FITCLEAN.MCD. For this calculation, the macro function g.fn was used:

g.fn
$=A R G U M E N T\left(g^{\prime \prime}\right)$
$=A R G U M E N T\left(I^{*}\right)$
$=A R G U M E N T\left(s^{*}\right)$
$=\left(L N\left(g / D+0.5^{*} s \wedge 2\right) / s\right.$
$=\left(L N(g /(I+1))+0.5^{*} s^{\wedge} 2\right) / s$
$=N O R M S D I S T(A 5)-N O R M S D I S T(A 6)$
$=$ RETURN(AT)

The calculated concentrations were subtracted from the field measured values to generate the residuals in columns 11 and 12. A second set of parameters, summarized in columns 14 and 13, were determined by trial and error to fit the residuals. The calculated values were plotted in embedded charts (not shown) in the spreadsheet, and the fit was judged visually.

In order to perform the final calculations for cumulative mass extracted and reduce the size of the spreadsheet, the operating time, observed TCE and PCE concentrations in ppm by volume, and early-time parameter values were exported to MASSFTT.XIS, a portion of which is shown in Fig. C.7.

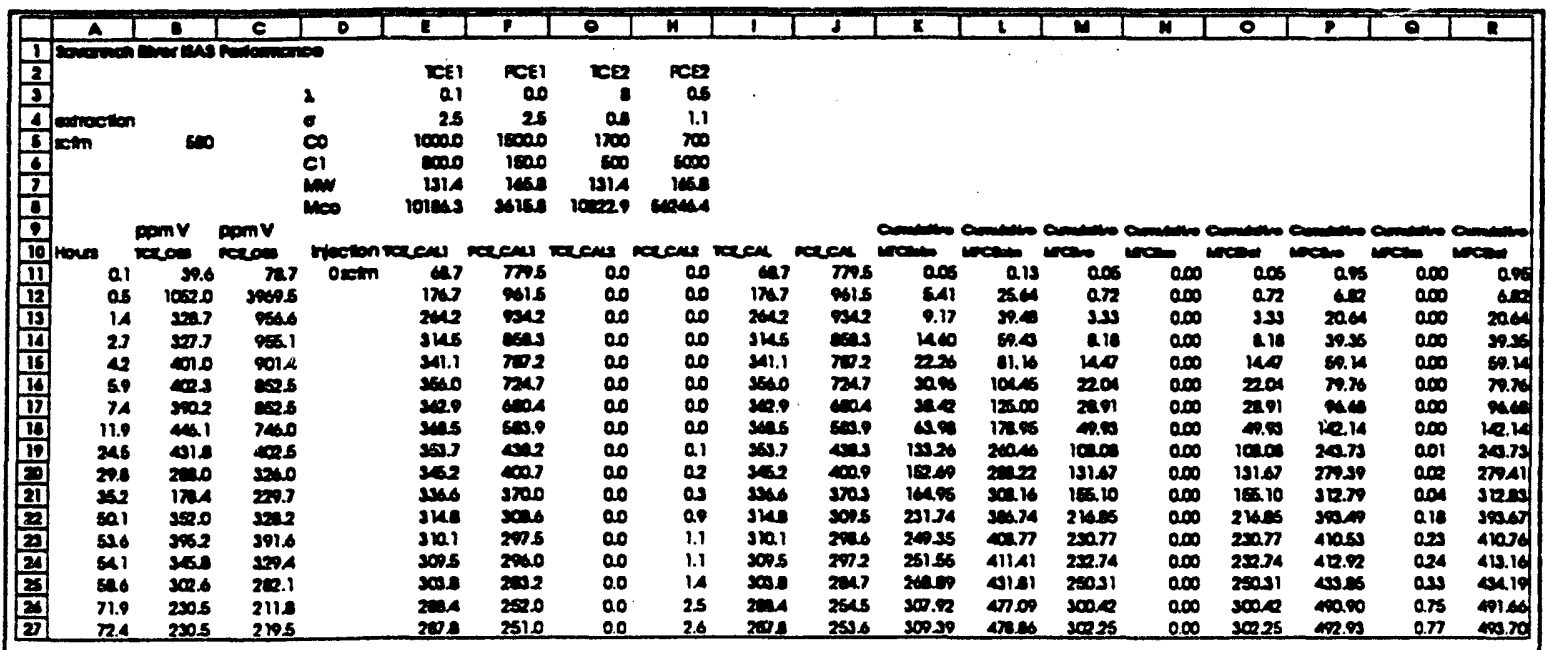

Figure C.7 Part of MASSFTT.XLS for final determination of heterogeneity model parameters for the Savannah River ISAS project.

Columns $\mathrm{A}, \mathrm{B}$, and $\mathrm{C}$ contain the field data. Columns $\mathrm{E}$ and $\mathrm{F}$ contain the calculated concentrat ons based on parameter set 1 (determined from the pre-injection data). Calculated results for parameter set 2 are given in Cols. $G$ and $H$. Columns $E$ and $G$ were added together to obtain the total calculated TCE concentrations given in Col. I, and F and $G$ were summed to obtain the total calculated PCE concentrations listed in Col. J. The macro g.fn was used again for calculating concentrations for parameter set 2 . The calculated and observed concentrations were shown previously in Fig. IV.15.

Field values of cumulative extraction were calculated separately for each contaminant from the reported volumetric concentrations and the nominal gas extraction rate of 580 SCFM, assuming that standard conditions refers to a pressure of $1 \mathrm{~atm}$. and a temperature of $32^{\circ} \mathrm{F}$. An alternative would be to use the reported volumetric rates for each time interval, but the average rate should suffice for the present 
no visually obvious difference in the quality of itt between these two examples, in spite of the very large differences in inventories and time scales.

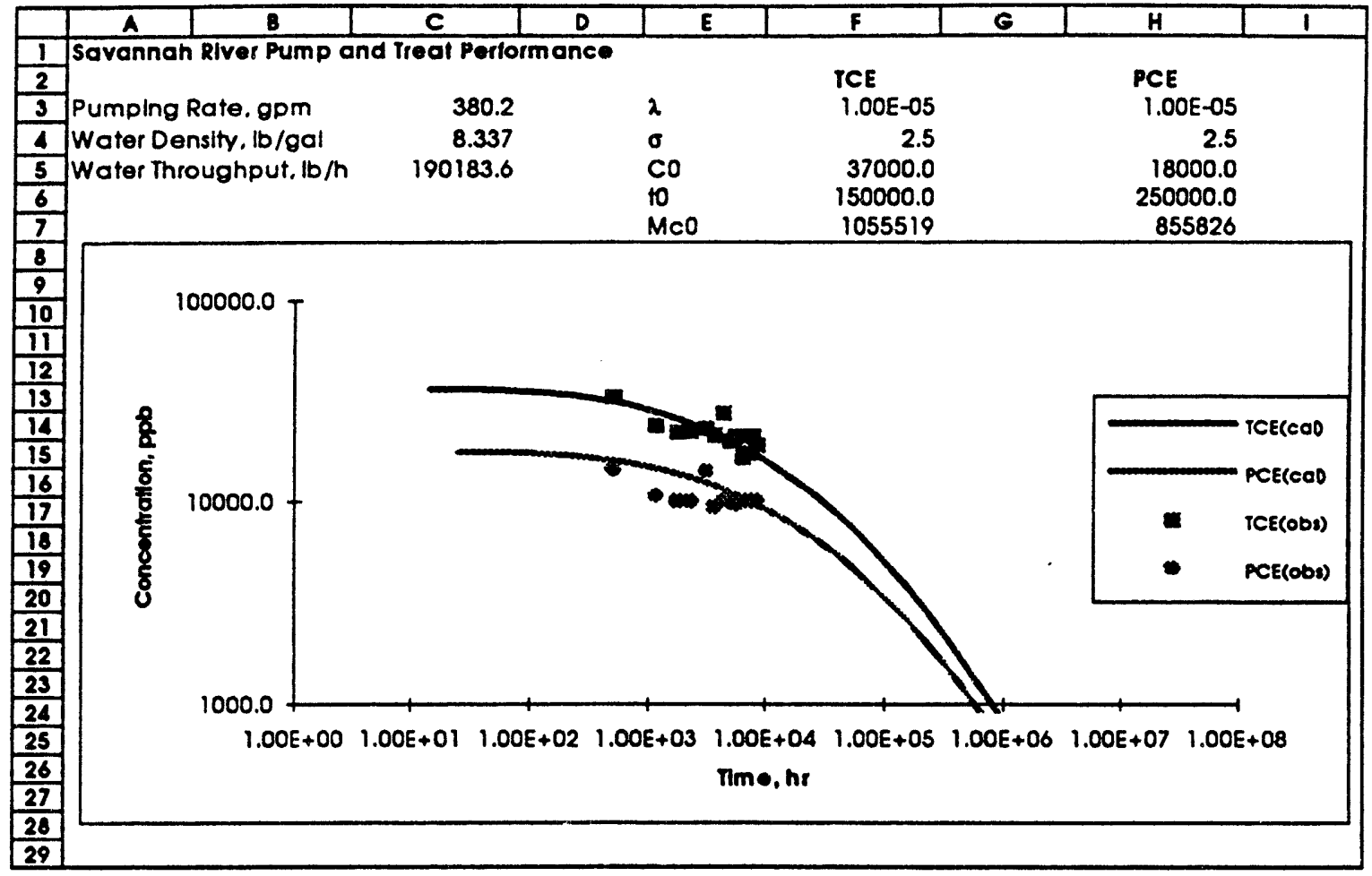

Figure C.8. First example of attempting to fit Savannah River Pump and Treat data with the heterogeneity parameter model.

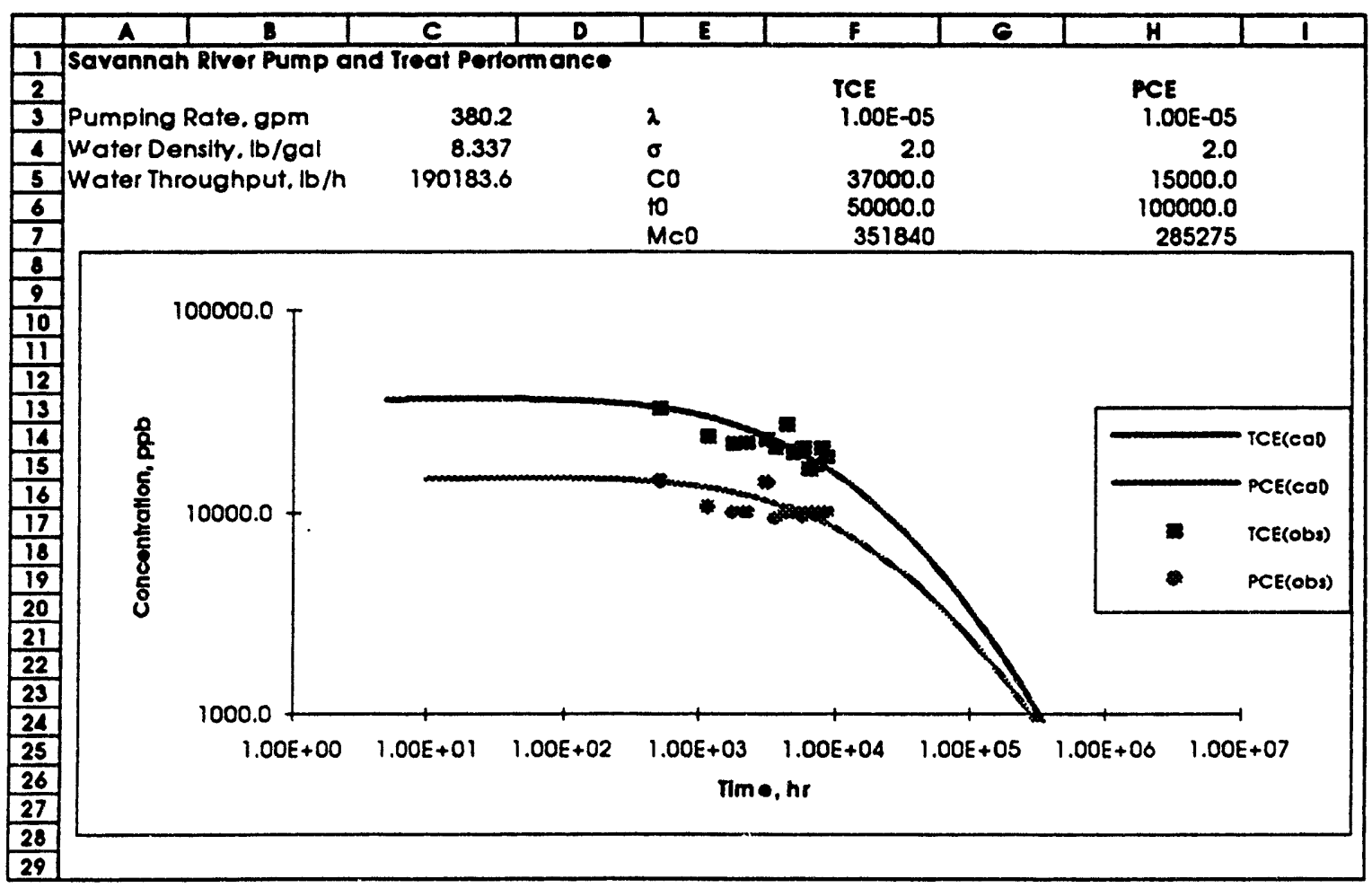

Figure C.9. Second example of attempting to fit Savannah River Pump and Treat data with the heterogeneity parameter model. 
In order to provide a more reproducible method of fitting the data, a second spreadsheet, PTFIT2.XIS, was developed. The same equation was used to calculate contaminant concentrations from assumed values of the parameters, and the RMS errors were calculated as well. For TCE, the RMS error increased from 795 for Example 1 to 930 for Example 2, while the error for PCE decreased from 588 to 437.

The parameters $c_{0}$ and $t_{0}$ were then systematically varied, while keeping $\lambda$ and $\sigma$ fixed for both contaminants, to produce the "best fit" values tabulated in Section IV. The RMS errors for TCE and PCE were reduced to 784 and 411 , respectively. Although these values represent at least local minima in the error surfaces, they may not be the lowest values which could be attained by more extensive trial-and-error calculations.

At some future date, it might prove worthwhile to try using a more sophisticated approach to minimizing the RMS error than simple trial and error. However, the error surface is highly non-linear, and one author's (Chesnut) previous experience in attempting to fit waterflood data by using steepest descent methods often led to physically unrealistic combinations of parameters. As a practical tool, it may well be better to provide a rapid means of calculating values and visually inspecting the agreement between calculated and observed results, while seeking a minimum in the error surface, than to attempt the development of a completely automated curve-fitting process.

Finally, a third spreadsheet, PT4CAST.XIS, was developed, using the parameter values obtained from fitting the observed data, to forecast concentration versus time and cumulative contaminant removal versus time, assuming that the operating conditions do not change. These results were shown in Fig. IV.20. 


\section{APPENDIX D. ANAlysis Of Flow Processes DURING TCE INFILTRATION IN HETEROGENEOUS SOILS AT THE SAVANNAH RIVER SITE, AIKEN, SOUTH CAROLINA ${ }^{1}$}

\section{INTRODUCTION}

Contamination of soils and groundwater from volatile organic compounds(VOCs), such as organic solvents and hydrocarbon fuels, is a problem at many industrial facilities. Key to successfully characterizing, containing, and eventually remediating the contamination is a thorough understanding, based on sound scientific principles, of the complex interplay of physical, chemical, and biological processes in geologic media, which affect the migration and distribution of the contaminants, and their response to remediation operations. A qualitative and quantitative understanding of contaminant behavior under natural conditions can provide valuable guidance for site characterization and monitoring. It can aid in evaluating the contamination that is likely to occur if no intervention is made ("no action" scenario), and it provides useful information for the design and implementation of remedial actions. Sound engineering design of containment and remediation of contaminants must be based on mechanistic models that adequately describe the important contaminant migration processes; such models must be calibrated and validated through appropriate site data.

This report focuses on physical mechanisms that affect contaminant behavior under the conditions encountered at the Savannah River site(SRS). Although other contaminants are present at the site, for the purpose of this discussion we will restrict ourselves to the processes following a spill and infiltration of trichloroethylene (TCE), which is the main contaminant present at the location of the Integrated Demonstration Project. We begin by briefly describing the main physical processes following release of TCE into the subsurface. Subsequently we will present simple engineering models that can help to evaluate contaminant migration processes in a semi-quantitative way. Finally, we will discuss results of detailed numerical simulations of TCE infiltration into a

\footnotetext{
1This Appendix was written by Karsten Pruess, Earth Sciences Division, Lawrence Berkeley Laboratory, as Lawrence Berkeley Laboratory report LBL-32418. It is reproduced here as received, except for the first page, which has been re-formatted to attain more consistency with the rest of this report. Pages have been re-numbered $D-1, D-2, D-3, \ldots$ in place of $1,2, \ldots$ in the original manuscript.
} 
heterogeneous medium consisting of sands and clays. These simulations attempt to shed light on the initial distribution of contaminants at the site prior to the start of remediation operations. We also point out limitations of present numerical modeling capabilities, and identify issues that require further research in order that a realistic description of contaminant behavior in the subsurface may be achieved.

\section{Contaminant Behavior under Natural Conditions}

The infiltration of TCE into the subsurface gives rise to a number of coupled multiphase partitioning and flow processes in a three-phase system consisting of an aqueous phase, a gas phase, and a non-aqueous phase liquid (NAPL). Table 1 lists some of the important thermophysical properties that determine contaminant behavior.

Table 1. Selected thermophysical properties of water, air, and TCE at ambient conditions of $20^{\circ} \mathrm{C}$ temperature, 1 bar pressure (as used in STMVOC code)

\begin{tabular}{|lccc|}
\hline \multicolumn{1}{|c}{ Component } & Water & Air & TCE \\
\hline \hline density $\left(\mathrm{kg} / \mathrm{m}^{3}\right)$ & 998.2 & 1.19 & 1462 \\
viscosity $(\mathrm{Pa} . \mathrm{s})$ & $1.00 \times 10^{-3}$ & $1.66 \times 10^{-5}$ & $.59 \times 10^{-3}$ \\
vapor pressure $(\mathrm{kPa})$ & 2.337 & - & 7.196 \\
\hline \multicolumn{4}{|c}{ effective density in phases $\left(\mathrm{kg} / \mathrm{m}^{3}\right)$} \\
\hline aqueous $\left(^{*}\right)$ & 997.2 & .0145 & 1.099 \\
gas $\left(^{*}\right)$ & .017 & 1.07 & .388 \\
NAPL & - & .003 & 1462 \\
\hline
\end{tabular}

$\left(^{*}\right)$ assuming that all three components are present

The density of TCE being approximately $1462 \mathrm{~kg} / \mathrm{m}^{3}$ at ambient conditions, as compared to $1.2 \mathrm{~kg} / \mathrm{m}^{3}$ for soil gas (humid air) and $998 \mathrm{~kg} / \mathrm{m}^{3}$ for water, a plume of NAPL will migrate downwards under the combined action of gravity, viscous, and capillary forces. Because of heterogeneities on different scales, such as clay lenses and depositional layers that differ in permeability, porosity, and capillary behavior, the NAPL will not flow straight downward in a piston-like displacement, but will instead be dispersed to varying degree both vertically and horizontally. Partial ponding may occur when the plume encounters regions of low permeability or adverse wettability, such as clays. The 
presence of aqueous and gas phases in the pore space will interfere with the NAPL migration (relative permeability effects). It will also impact on the NAPL by way of phase partitioning processes, namely, dissolution in the aqueous phase, and evaporation into the gas phase. At ambient conditions of $\mathrm{T}=20^{\circ} \mathrm{C}, \mathrm{P}=1 \mathrm{bar}$, the partial density of $T C E$ in a saturated aqueous solution is $1.10 \mathrm{~kg} / \mathrm{m}^{3}$, while the density of saturated TCE vapor is $.39 \mathrm{~kg} / \mathrm{m}^{3}$. Thus, the amount of TCE in $1 \mathrm{~m}^{3}$ of free product, $1462 \mathrm{~kg} / \mathrm{m}^{3}$, is equal to the TCE content in $1330 \mathrm{~m}^{3}$ of saturated aqueous solution, and $3768 \mathrm{~m}^{3}$ of vapor-saturated gas. The TCE can also sorb on solid phases, such as organic carbon and soil minerals. This sorption may be partially or completely reversible, and it may occur instantaneously (equilibrium sorption) or be controlled by kinetic rates. Portions of the NAPL plume may also be immobilized in the pore space by capillary force.

The downward advancement of a TCE plume is an intrinsically unstable process, because higher-density fluid (TCE) is present above fluids of lower density (water, soil gas). Interacting with ever present soil heterogeneities on different scales, the gravitational instability may give rise to a highly dispersed displacement front (fingering). Because TCE is denser than water, a plume of TCE will continue to sink downward below the water table, and will tend to migrate to deep and poorly accessible regions.

The presence of TCE vapors considerably increases the density of soil gas. At ambient conditions of $\mathrm{T}=20^{\circ} \mathrm{C}, \mathrm{P}=1 \mathrm{bar}$, the density of (humid) air is $1.18 \mathrm{~kg} / \mathrm{m}^{3}$, while the density of air saturated with TCE vapor is $1.48 \mathrm{~kg} / \mathrm{m}^{3}$. This density contrast provides a negative (downward) buoyancy force which can induce large-scale gas phase convection with associated spreading of the contaminant (Falta et al., 1989). Additional mechanisms for contaminant spreading through gas phase processes include molecular diffusion, and barometric pumping.

\section{Gravity-driven Flow of a NAPL Plume}

Neglecting effects from capillary and pressure forces, the flux in a NAPL plume that is falling freely under gravity in the vadose zone can be estimated from a multiphase version of Darcy's law as

$$
F_{n}=k \frac{k_{1 n}}{\mu_{n}} \rho_{n}^{2} g
$$

Here $F_{n}$ is mass flux in $\mathrm{kg} / \mathrm{s} \mathrm{m}^{2}, \mathrm{k}$ and $\mathrm{k}_{\mathrm{r} n}$ are absolute and relative permeability, respectively, $\rho_{n}$ is NAPL density, $\mu_{n}$ is NAPL viscosity, and $g$ is the acceleration of gravity. In writing Equation (1) we have neglected the small density of soil gas relative to liquid NAPL. Inserting values of $\rho_{n}=1462 \mathrm{~kg} / \mathrm{m}^{3}$ and $\mu_{n}=0.59 \times 10^{-3}$ Pa.s appropriate for 
TCE, Equation (1) gives

$$
\mathrm{F}_{\mathrm{n}}=\mathrm{k} \cdot \mathrm{k}_{\mathrm{rn}} \times 3.554 \times 10^{10} \quad(\mathrm{~kg} / \mathrm{s})
$$

If NAPL is spilled at a mass rate $Q(\mathrm{~kg} / \mathrm{s})$, the horizontal cross sectional area $A_{Q}$ to which the plume will spread can be estimated from

$$
Q=A_{Q} \cdot F_{n}
$$

Substituting from Equation (1) we have

$$
A_{Q}=\frac{\mu_{n} Q}{k k_{n n} \rho_{n}^{2} g}
$$

Inserting parameters appropriate for TCE, this gives

$$
A_{Q}=\frac{1.81 \times 10^{-11}}{k_{\text {rn }}} Q \quad\left(\mathrm{~m}^{2}\right)
$$

A similar discussion can be made for gravity-driven migration of NAPL below the water table. In this case the effective body force on the NAPL is $\left(p_{n}-\rho_{w}\right) * g$, so that the flux can be estimated as

$$
F_{n}=k \frac{k_{n n}}{\mu_{n}} \rho_{n}\left(\rho_{n}-\rho_{w}\right) g
$$

which is a factor $\left(p_{n}-\rho_{w}\right) p_{n}=(1462-998) / 1462=.32$ smaller than the corresponding expression Equation (1) for the vadose zone.

Suppose that a TCE plume advances downward by building to a saturation $S_{\mathrm{TCE}}=1$ $-S_{w r}$ The rate at which the TCE plume advances can then be estimated from

$$
\phi\left(1-S_{w x}\right) p_{n} u_{n}=F_{n}
$$

Here $u_{n}$ is the actual (pore) velocity of TCE advancement. For plume propagation in the vadose zone we have, from Equations (1) and (7)

$$
u_{n}=k k_{1 n} \frac{\rho_{n} g}{\phi\left(1-S_{w r}\right) \mu_{n}}
$$

\section{NAPL Mobilization by Buoyant Gas Flow}

Buoyant gas flow due to the presence of a volatile organic compound (VOC) such as TCE was discussed by Falta et al. (1989). The Darcy velocity (volumetric flux) of downward gas flow can be estimated in analogy to Equations (1) and (6) as 


$$
v_{g a s}=k \frac{k_{g g}}{\mu_{g}}\left(\rho_{g}-\rho_{\text {air }}\right) g
$$

Here $\rho_{g}$ is the density of gas containing a mixture of (moist) air and VOC vapor, while $\rho_{\text {air }}$ is the density of ambient soil gas. The VOC mass flux associated with the volumetric flux of Equation (9) is obtained by multiplying with the partial density $\rho_{\mathrm{gn}}$ of contaminant vapors in the gas phase. In the presence of free NAPL phase the appropriate density is the saturated vapor density $\rho_{\mathrm{gn}}^{0}$ which from the ideal gas law can be estimated as

$$
\rho_{\mathrm{gn}}^{0}=\frac{\mathrm{P}^{0} \mathrm{M}}{\mathrm{RT}}
$$

where $\mathrm{P}^{0}$ is the saturated vapor pressure of the VOC, $M$ is the molecular weight, $R$ the universal gas constant, and $\mathrm{T}$ the absolute temperature. Adopting ideal-gas approximations also for the densities of air and air/vapor mixtures, Equations (9) and (10) combine to yield a VOC vapor flux

$$
E=k k_{\tau g} \frac{g}{\mu_{g}}\left[\frac{P^{0}}{R T}\right]^{2} M\left(M-M_{\text {air }}\right)
$$

We have denoted the buoyant downward mass flux of VOC vapors by $\mathrm{E}$, because, under presumed conditions of local phase equilibrium, it must equal the evaporation rate of free NAPL phase per unit horizontal cross sectional area. At $\mathrm{T}=20^{\circ} \mathrm{C}$, saturated vapor pressure of TCE is $7.2 \mathrm{kPa}$, and air viscosity is $1.66 \times 10^{-5} \mathrm{~Pa}$.s, so that from Equation (11) we have

$$
E=k k_{\mathrm{rg}} \times 6.94 \times 10^{4}\left(\mathrm{~kg} / \mathrm{m}^{2} \cdot \mathrm{s}\right)
$$

Assuming that NAPL is present at irreducible saturation $S_{n \text { r }}$, the thickness of NAPL layer removed per unit time from buoyant gas flow, $\dot{\mathrm{d}}_{\mathrm{g}}$, can be estimated from

$$
E=\phi S_{n x} \rho_{n} \dot{d}_{g}
$$

Inserting typical parameters of $\mathrm{k}_{\mathrm{rg}}=1 . \times 10^{-11} \mathrm{~m}^{2}, \phi=.4, S_{\mathrm{nr}}=.05, \rho_{\mathrm{n}}=1462 \mathrm{~kg} / \mathrm{m}^{3}$, we have $\dot{d}_{g}=2.38 \times 10^{-8} \mathrm{~m} / \mathrm{s}=.75 \mathrm{~m} / \mathrm{yr}$, which represents a significant amount of contaminant removal.

\section{NAPL Leaching by Water}

An analysis similar to the above can be made for removal of free NAPL phase by means of leaching from infiltrating water. An infiltrating volumetric water flux $v_{w}$ will dissolve NAPL at a rate of 


$$
D=v_{w} \rho_{w n}
$$

where $\rho_{w n}$ is the partial density of NAPL in saturated aqueous solution. Again assuming that NAPL is present at irreducible saturation $S_{n r}$ the thickness of NAPL layer removed per unit time from dissolution in infiltrating groundwater, $\dot{\mathrm{d}}_{w}$, can be estimated from

$$
D=\phi S_{u} \rho_{n} \dot{d}_{w}-S-z-u b-n r
$$

It is of interest to compare the rates of NAPL removal by buoyant gas flow and dissolution in infiltrating ground water. For parameters applicable to TCE we have, from Equations (12) and (14)

$$
\frac{E}{D}=\frac{k k_{r g}}{v_{w}} \times \frac{6.94 \times 10^{4}}{1.1}=63.09 \times 10^{3} \frac{k k_{r g}}{v_{w}}
$$

For a hypothetical water infiltration rate of $1 \mathrm{~m} / \mathrm{yr}$, this ratio becomes

$$
\left[\frac{E}{D}\right]_{v_{-}=1 \mathrm{~m} / \mathrm{yr}}=1.991 \times 10^{12} \mathrm{k} \mathrm{k}_{\mathrm{rg}}
$$

so that evaporation of NAPL into gas phase flowing under buoyancy would equal dissolution into infiltrating groundwater at an effective gas permeability of $1 / 1.991 \times 10^{12} \mathrm{~m}^{2}$ $=.55 \times 10^{-12} \mathrm{~m}^{2}$, or .55 darcy. For higher permeability evaporation would dominate, while for lower permeability dissolution would be a more important mechanism for NAPL removal.

\section{Gas Diffusion}

The spreading of VOC vapors in the gas phase by molecular diffusion has been discussed by Falta et al. (1989). It is described by a diffusion equation

$$
\frac{\partial X_{g}^{c}}{\partial t}=D_{g, e f f} \Delta X_{B}^{c}
$$

where $\mathrm{X}_{\mathrm{g}}^{c}$ is the mass fraction of VOC vapor in the gas phase, and the effective gas diffusivity is given by

$$
\mathrm{D}_{\mathrm{g}, \mathrm{eff}}=\frac{\tau}{\mathrm{R}_{\mathrm{g}}} \mathrm{D}_{\mathrm{g}}
$$

Here, $\tau$ is a tortuosity factor, typically of order 0.5 , and $R_{g}$ is the gas phase retardation factor, which accounts for phase partitioning of VOC vapors into liquid and solid phases through dissolution and sorption, respectively. The retardation factor is

$$
R_{g}=1+\frac{S_{w}}{S_{g} H}+\frac{\rho_{g} K_{D}}{\phi S_{g} H}
$$


Here

$$
\mathrm{H}=\frac{\mathrm{C}_{\mathrm{g}}}{\mathrm{C}_{\mathrm{w}}}
$$

is Henry's constant, which is the ratio of effective densities (concentrations) $C_{g}=\rho_{g}$ * $X_{g}^{c}$ and $C_{w}=\rho_{w} * X_{w}^{c}$ of the contaninant in gas and water phases, respectively.

$$
K_{D}=\frac{X_{s}^{c}}{C_{w}}=K_{o c} f_{o c}
$$

is the distribution coefficient for the VOC chemical between solid and aqueous phases, given by the ratio of mass fraction of adsorbed VOC in the solid phase, $\mathrm{X}_{\mathrm{s}}^{\mathrm{c}}$, to effective density of VOC dissolved in the aqueous phase. This is usually assumed to be proportional to fraction of organic carbon, $\mathrm{f}_{o c}$ in the soil, the proportionality factor $\mathrm{K}_{o c}$ being referred to as the organic carbon partition coefficient.

For TCE, we have $\mathrm{H}=.388 / 1.099=.353$. Assuming that adsorption is insignificant at Savannah River (Eddy et al., 1991), the gas phase retardation factor for a typical irreducible water saturation of $S_{w r}=.15$ is approximately 1.5. For a typical "free" gas diffusivity of $10^{-5} \mathrm{~m}^{2} / \mathrm{s}$, the effective gas diffusivity from Equation (19) then becomes $3.3 \times 10^{-6} \mathrm{~m}^{2} / \mathrm{s}$. To put this number in perspective it may be compared with a typical thermal diffusivity for heat conduction in soils, which is $D_{\text {th }}=K / \rho * c=1.5 / 2500 * 1000$ $=0.6 \times 10^{-6} \mathrm{~m}^{2} / \mathrm{s}$. The distance to which diffusive spreading reaches can be estimated as

$$
x=\sqrt{D t}
$$

Thus, diffusive spreading of TCE in the gas phase is somewhat more rapid (by a factor $\sqrt{3.3 / 0.6}=2.3$ ) than conductive heat transfer in soils, but it is a relatively slow process. Based on the effective gas phase diffusivity of $3.3 \times 10^{-6} \mathrm{~m}^{2} / \mathrm{s}$ for TCE estimated above, diffusive penetration distances are $.53 \mathrm{~m}$ in 1 day, $10.2 \mathrm{~m}$ in 1 year, and $32.3 \mathrm{~m}$ in 10 years.

\section{Barometric Pumping}

VOC vapors may be removed from the subsurface as a result of atmospheric pressure variations, a process that we shall refer to as "barometric pumping." Atmospheric pressure is subject to fluctuations with a dominant cycle period of 24 hours. As atmospheric pressure rises, the column of soil gas above the water table is compressed, and "clean" atmospheric air enters the subsurface, where it may acquire some VOC contamination through processes such as mixing with already contaminated soil gas, evaporation 
of resident NAPL, or diffusion of VOC vapors down concentration gradients. When atmospheric pressure subsequently decreases, the soil gas column expands, discharging a certain amount of contarninant to the atmosphere.

The quantitative aspects of this process may be estimated in analogy to the problem of moisture removal from the vadose zone by barometric pumping (Tsang and Pruess, 1989). From the ideal gas law, decrease of atmospheric pressure from $P \rightarrow P-\Delta P$ will result in an increase in the height of the soil gas column from $H \rightarrow H+\Delta H$ such that

$$
\frac{\Delta \mathrm{P}}{\mathrm{P}}=\frac{\Delta \mathrm{H}}{\mathrm{H}}
$$

Per unit surface area, the amount of VOC vapor removed in one full pressure cycle will be

$$
M_{V O C}=\Delta H \phi S_{g} \rho_{g n}=H\left[\frac{\Delta P}{P}\right] \phi S_{g} \rho_{g n}
$$

For an order-of-magnitude estimate, we use $\triangle P / P=.005$ (Tsang and Pruess, 1989), and $H$ $=40 \mathrm{~m}, \phi=.4, S_{g}=.8$, to obtain a removal of VOC vapors of $.064 * \rho_{\mathrm{gn}} \mathrm{kg} / \mathrm{m}^{2}$ per pressure cycle (one day). Making the extreme assumption that VOC vapor in soil gas beneath the land surface is at saturated density of $.39 \mathrm{~kg} / \mathrm{m}^{3}$, this translates into a removal of 788 $\mathrm{kg} / \mathrm{m}^{2}$ per year, a very large amount. Realistically, however, VOC vapor concentration will likely be much less than saturated near the ground surface, so that contaminant removal rates from atmospheric pumping will be considerably less. For the parameters assumed above, the thickness of soil layer beneath the ground surface that is directly affected by barometric pumping is only $\Delta \mathrm{H}=.005 * \mathrm{H}=0.2 \mathrm{~m}$. After removal of free NAPL phase from this zone immediately beneath the ground surface, VOC vapors can be supplied to the atmospheric pumping process only by diffusion from below (as convective transport is downward). The long-term impact of atmospheric pumping thus seems to be to effectively shift the atmosperic "zero VOC concentration" boundary downward by a small distance of order $\Delta \mathrm{H}=0.2 \mathrm{~m}$, which is not expected to have a significant effect on contaminant migration.

\section{Numerical Simulation of TCE Infiltration}

Some of the important processes affecting contaminant migration and dispersal may operate on relatively small spatial scales $(<1 \mathrm{~m})$, which may not be resolvable with the kind of spatial discretization that would normally be employed in engineering models of a site. For example, observations at the Savannah River site indicate that TCE contamination is highly spatially variable, and tends to be localized near the top of clay-rich 
zones (Eddy et al., 1991). The strong heterogeneity of contaminant distribution has important implications for the feasibility and success of remediation operations. We need to understand why the contaminant is distributed in the observed manner, and what the driving forces and conditions are that have generated this kind of distribution. Only if we identify the processes leading to the observed contaminant distribution can we hope to be able to perform a reliable evaluation of remediation schemes, and to develop an understanding of what the processes can and cannot accomplish in a given time frame. Such process understanding is also essential for a transfer of the experience gained at Savannah River to other sites.

In this section we report on the current status of our efforts to use multiphase numerical simulation as a tool for developing a better understanding of the role of formation heterogeneity in contaminant transport. In order to be able to identify the manner in which the different multiphase flow and partitioning processes interact with formation heterogeneity we have simulated idealized "generic" models, which were designed to capture important features of the Savannah River site, such as layering of highly permeable sands with clays of variable horizontal extent. Rather than modeling the full complement of multiphase processes in a complex heterogeneous setting, we introduce process and formation complexity in a step-wise fashion, to be able to discern the important controls on contaminant behavior. For example, we examine in considerable detail the interaction of a descending TCE plume with clay lenses and clay layers of different spatial extent, both in the vadose zone and beneath the water table. It is hoped that the insight gained from such detailed studies will eventually support a scale-up to "effective" process description on a larger scale.

Numerical modeling of TCE infiltration is subject to space discretization effects (numerical dispersion), which can be particularly severe because of the gravitationally unstable nature of the process (Pruess, 1991). These effects can give rise to spurious (unphysical) flows, which may depend strongly on size and orientation of the numerical grid used. We have performed a grid orientation study to specifically address these isssues (below).

\section{TOUGH2 and STMVOC Codes}

The simulations reported here were carried out with the TOUGH2 and STMVOC simulators, which are closely related members of the TOUGH/MULKOM family of multiphase simulation codes, developed at Lawrence Berkeley Laboratory. TOUGH2, a successor to TOUGH, is a general-purpose simulator for nonisothermal flows of multiphase, 
multicomponent fluids (Pruess, 1987, 1991). STMVOC, a descendent of TOUGH, features the same general architecture and solution methodology (Falta and Pruess, 1991; Falta et al., 1992a). It is a specialized code for nonisothermal flow of three-phase threecomponent mixtures of water, air (a pseudo-component) and a volatile organic compound (VOC). STMVOC describes all of the multiphase processes discussed above, including full phase partitioning of the VOC between NAPL, gas, aqueous, and solid phases, VOC migration by convection in all of the three phases, and VOC diffusion in the gas phase. It has full capabilities to describe strongly heat-driven processes, such as steam drive of volatile contaminants. However, in the present study STMVOC has been used only for processes at ambient temperature of $20^{\circ} \mathrm{C}$. The code has been validated by comparison with laboratory experiments (Falta et al., 1992b).

\section{Model System}

The model system is loosely patterned after the conditions at the site of the SRS Integrated Demonstration Project (see Figures 1 and 2). The main contaminant source being a process sewer line of considerable horizontal length, we model a 2-D vertical $(\mathrm{X}-\mathrm{Z})$ section perpendicular to the sewer line. Constant gas pressure conditions are maintained at the ground surface, and constant gas and aqueous phase pressures are maintained at the distant lateral boundaries. The water table is placed at a depth of approximately $130 \mathrm{ft}$ (Eddy et al., 1991). Total vertical extent of the system modeled is $160 \mathrm{ft}$, at which depth boundary conditions of "no flow" or "constant pressure" are imposed.

For the simulations reported below STMVOC was run in isothermal mode, with the entire flow system held at a temperature of $20^{\circ} \mathrm{C}$ by means of assignment of a very large heat capacity to the porous medium.

Some of the parameters that have an important impact on the behavior of the threephase water-air-NAPL system are poorly known. This is especially true for the "characteristic curves" (relative permeability and capillary pressure curves). For relative permeabilities we have adopted a functional relationship developed by Stone (1970) that is widely used in petroleum reservoir engineering. This relationship had to be slightly modified to deal with subtle issues of phase behavior near irreducible saturations. The equations for water, gas, and NAPL phase relative permeability used here are:

$$
\begin{aligned}
& k_{r w}=\left[\frac{S_{w}-S_{w r}}{1-S_{w r}}\right]^{n} \\
& k_{r g}=\left[\frac{S_{g}-S_{g r}}{1-S_{w r}}\right]^{n}
\end{aligned}
$$




$$
k_{r n}=\left[\frac{1-S_{g}-S_{w}-S_{n r}}{1-S_{g}-S_{w r}-S_{n r}}\right]\left[\frac{1-S_{w r}-S_{n t}}{1-S_{w}-S_{n r}}\right]\left\{\frac{\left(1-S_{g}-S_{w r}-S_{n r}\right)\left(1-S_{w}\right)}{\left(1-S_{n r}\right)}\right\}^{n}
$$

The modification made concerns the term in the curly brackets for $k_{n} n$, where we have replaced Stone's expression $\left(1-S_{g}-S_{w r}\right)$ by $\left(1-S_{g}-S_{w r}-S_{n x}\right)$, to avoid the unphysical possibility of obtaining large $k_{f n}$ near $S_{n x}$ when $S_{g}$ is near $1-S_{w r}-S_{n r}$.

For capillary pressures we used the three-phase relationships given by Parker et al. (1987); however, capillary pressure between gas and NAPL phases was neglected. The capillary pressure between the aqueous phase and the non-wetting gas and NAPL phases is

$$
P_{c g w}=-\frac{\rho_{w g}}{\alpha_{n w}}\left[\left(\frac{1-S_{m}}{S_{w}-S_{m}}\right)^{\frac{n}{n-1}}-1\right]^{1 / n}
$$

A summary of problem specifications intended to be representative of conditions at the site of the Savannah River integrated demonstration project is given in Table 2. Capillary pressures for different hydrogeologic units were scaled inversely to the square root of absolute permeability.

\begin{tabular}{|c|c|c|c|c|}
\hline & & Sands & Clayey Sands & Clays \\
\hline $\begin{array}{l}\text { permeability }\left(\mathrm{m}^{2}\right) \\
\text { porosity }\end{array}$ & $\begin{array}{l}\mathbf{k} \\
\phi\end{array}$ & $\begin{array}{c}10^{-11} \\
.35\end{array}$ & $\begin{array}{c}10^{-12} \\
.35\end{array}$ & $\begin{array}{c}10^{-15} \\
.50\end{array}$ \\
\hline \multicolumn{5}{|c|}{ relative permeabilities (Equations 26-28) } \\
\hline $\begin{array}{l}\text { irreducible water saturation } \\
\text { irreducible gas saturation } \\
\text { irreducible NAPL saturation } \\
\text { exponent }\end{array}$ & $\begin{array}{l}S_{w r} \\
S_{g r} \\
S_{\mathrm{mr}} \\
n\end{array}$ & $\begin{array}{l}.15 \\
.001 \\
.05 \\
3\end{array}$ & $\begin{array}{l}.60 \\
.001 \\
.05 \\
3\end{array}$ & $\begin{array}{c}.60 \\
.001 \\
.05 \\
3\end{array}$ \\
\hline \multicolumn{5}{|c|}{ capillary pressures (Equation 29) } \\
\hline $\begin{array}{l}\text { strength paral veter } \\
\text { limiting saturation } \\
\text { exponent }\end{array}$ & $\begin{array}{c}\alpha_{n w} \\
S_{m} \\
n\end{array}$ & $\begin{array}{l}5.0 \\
0.0 \\
1.84\end{array}$ & $\begin{array}{l}1.58 \\
0.0 \\
1.84\end{array}$ & $\begin{array}{l}.05 \\
0.0 \\
1.84\end{array}$ \\
\hline
\end{tabular}

Table 2. Formation Properties for 2-D Vertical Section Problem

The flow domain is a $1 \mathrm{~m}$ thick vertical section, with dimensions of $160 \mathrm{ft}$ in the vertical, 
$1000 \mathrm{ft}$ horizontally. For numerical simulation it is discretized into 24 rows and 15 columns of varying spacing (Figure 3). Finer vertical discretization was employed at the elevations at which clays are encountered at the site, to be able to better resolve flow processes associated with those clays. Horizontal discretization is finest near the assumed symmetry line (left hand side) where TCE release is taking place. An expanded view of a part of the calculational mesh is shown in Figure 4, which also indicates the assignment of domains with different hydrologic properties. The domains labeled "325CL" and "300CL," respectively, correspond to the $325 \mathrm{ft}$ and $300 \mathrm{ft}$ clays encountered at the site (Figure 1), and are assigned clay properties as given in Table 2. The domain "TANCL" represents a clayey sand corresponding to the "tan clay" at the site. It should be emphasized that in its present form the assignment of these units and their hydrologic properties is rather schematic. They are meant to represent the hydrogeologic features of the site in a generic way, so that important process aspects may be explored. The description of hydrogeologic units and the specification of their properties needs to be refined in the future, so that natural conditions and remediation response may be modeled in more realistic detail.

Prior to startup of TCE infiltration, the system was run to gravity-capillary equilibrium. Water saturation in the vadose zone was assumed to be at irreducible levels. For the initial set of runs no water recharge from the surface was taken into account. The contaminant is assumed to be pure TCE with thermophysical properties as given for problem 3 in the STMVOC User's Guide (Falta and Pruess, 1991). Release of contaminant is modeled by injecting TCE into the uppermost grid block at the left hand side of the model, at a rate of $89.68 \times 10^{-6} \mathrm{~kg} / \mathrm{s}$, which is equivalent to 1 barrel per 30 -day month. Because of symmetry we only model half of the domain, so that the spill rate amounts to 2 barrels of TCE per month per meter of sewer line.

\section{Infiltration into Clayey Sands}

We have modeled TCE infiltration over a time period of 30 years. For the following discussion of the behavior of the NAPL plume refer to Figures 5 and 6, which show NAPL saturation distributions after 3 years of infiltration in a large-scale and a close-up view, respectively.

In response to TCE release, NAPL saturation in the injection grid block builds up until the residual NAPL saturation of $5 \%$ is exceeded, at which point the NAPL becomes mobile and begins to flow downward under gravity. Eventually the descending NAPL plume encounters a clay lense (the " $325 \mathrm{CL}$ " domain, Figure 4), which because of its low absolute and relative permeability acts as an obstacle to downflow of NAPL. 
Ponding with buildup of NAPL saturation takes place, which is accompanied by modest pressure increase. This causes most of the NAPL to flow laterally, but a small amount of NAPL is slowly invading the clay zone itself, and ultimately passes right through it. The NAPL flowing laterally to circumvent the clay lense resumes its predominant downward flow after it reaches a break in the clay lense, and subsequently ponds again as it encounters the more extensive clay zone at $60 \mathrm{ft}$ depth (the $300 \mathrm{ft}$ clay). The growth of the ponded zone slows with time, as an increasing proportion of the infiltrating TCE penetrates into the clay. The extent of the ponded zone has almost stabilized after 10 years (compare Figures 7-9), eventually reaching an area of $38.1 \mathrm{~m}^{2}$. From Equation (5) the ponded area may be estimated as $A_{Q}=2.52 / \mathrm{k}_{\mathrm{nn}} \mathrm{m}^{2}$, which is consistent with the simulation results, but also indicates that because of the strong dependence of $A_{Q}$ on the a priori unknown $k_{r n}$, Equation (5) is of limited utility in that it can only provide a very conservative lower bound. It is evident that a considerably broadened plume descends below the clay zone. After 30 years the plume has penetrated all the way into the water table, and NAPL saturation is beginning to build up at the bottom of the flow system, which was assumed impermeable in this simulation (see Figure 9).

In addition to a free-phase NAPL plume, a plume of TCE vapors develops in the gas phase (Figures 10-14). This plume spreads much more quickly to a large volume, because of gas phase convection driven by the negative buoyancy of vapor-ladden soil gas. From the shape of the concentration contours one can clearly see how the downward convection is diverted sideways as the lower permeability of the "TANCL" domain is encountered at a depth of $90 \mathrm{ft}$. The regions enclosed by the $0.3 \mathrm{~kg} / \mathrm{m}^{3}$ concentration contour correspond closely to the extent of the NAPL plume, as concentrations approaching the saturated value of $0.388 \mathrm{~kg} / \mathrm{m}^{3}$ can only be maintained in immediate proximity to free NAPL. Note also that, wherever TCE vapor is present, a TCE concentration in equilibrium with the gas phase concentrations is maintained in the aqueous phase. The amount of TCE dissolved per unit volume of aqueous phase is a factor 2.83 larger than what is present in the gas phase.

Diffusive transport of TCE vapors in the gas phase was neglected in the present calculation. As was discussed here diffusion will yield a migration distance of order $100 \mathrm{ft}$ over 10 years, adding further spreading of the TCE vapor plume which is not insignificant, but is considerably smaller than the distance covered by advective transport. The advective migration of TCE vapors is probably overestimated in the simulation presented here, because of the rather small extent of clay zones in the model. The presence of more extensive clay layers with only limited breaks at Savannah River would tend to reduce gas convection effects. 
Another simulation was run in which TCE infiltration was stopped after 10 years, and the system was left alone to examine the redistribution of contaminant that would occur under natural "no action" conditions. The NAPL plume after 10 years of infiltration followed by 20 years of no infiltration is shown in Figure 15, which should be compared to Figure 7 for the plume at 10 years. It is seen that the NAPL plume has continued to disperse outward and downward, while highest NAPL saturations have been reduced. There is a rather limited region above the clay lense where the NAPL has been removed by persistent evaporation into the descending gas stream. This simulation was continued to 100 years total time (results not shown), after which time NAPL was completely swept out of the region above the clay lense, with very significant NAPL saturations remaining in the clay lense itself (approximately $S_{n}=12 \%$ ). A similar pattern of behavior was seen at the more extensive clay layer. This can be understood by noting that gas convection is confined to the highly permeable sands. Thus, although penetration of NAPL into the clays is inhibited by low absolute and relative permeability, NAPL that will get into the clays is little affected by gas phase redistribution processes.

A brief exploration of flow system behavior in the presence of water infiltration from precipitation was also made. Applying a recharge rate of 15 inch/year (Colven et al., 1987), the system was first run to steady state. This resulted in a modest increase in water saturations beyond irreducible levels in the highly permeable sands (from 15\% to approximately $19 \%)$, while the clays became almost completely water-saturated $\left(S_{w}\right\rangle$ 98\%). Steady water saturations for the case with recharge are shown in Figure 16. The attainment of nearly full water saturation by the clays can be understood by noting that the permeability of the clay units of $10^{-15} \mathrm{~m}^{2}$ corresponds to a hydraulic conductivity of approximately $10^{-8} \mathrm{~m} / \mathrm{s}$, or $.32 \mathrm{~m} / \mathrm{yr}$, which is slightly less than the applied recharge rate. Figure 16 shows that there is some increase of water saturation above the clays; part of the infiltrating flux is being diverted around the clay zones. After a steady state with 15 inch/ year of recharge was reached, TCE was injected at the same rate as in the previous set of simulations without water infiltration. The NAPL plume after 10 years is shown in Figure 17; it looks very different from the previous case of no water infiltration (Figure 7). The NAPL again ponds atop the clays, but due to their nearly complete water saturation the clays now act as nearly impermeable barriers to the NAPL, and very little NAPL penetration into the clays themselves takes place. Virtually all of the injected TCE is being diverted laterally around the clays. However, in the aqueous phase in the clay regions, TCE is present at close to saturated concentrations. 


\section{Grid Orientation Effects}

In simulations of oil recovery operations involving two- or three-phase flow it has been noticed for a long time that predicted results can be sensitive to the orientation of the computational grid used. Mobilization of highly viscous oil by less viscous water or steam is an "unfavorable mobility ratio" displacement, which is subject to a hydrodynamic instability (viscous fingering). The finite space discretization used in finite difference or finite element numerical simulations generates a purely numerical (as opposed to physical) dispersion with associated spurious flows. Numerical dispersion in general will be anisotropic and tends to be strongest in the directions of the lines of the numerical grid, and weakest in the directions running diagonally to the grid. The "grid orientation effect" (spurious dependence of simulated results on the orientation of the numerical grid) arises from an interplay between hydrodynamic instability and anisotropic numerical diffusion (Brand et al., 1991).

In the context of oil recovery simulations, grid orientation effects have been discussed for horizontal flows, with particular focus on steam flooding (Todd et al., 1972; Coats et al, 1974; Coats, 1982; Coats and Ramesh, 1984). The customary approach has been to consider a five-spot production-injection arrangement, and to compare simulations with "parallel" and "diagonal" grids (Figure 18). It has been shown that grid orientation effects can be substantially reduced or eliminated by means of higher-order differencing schemes, which maintain a higher degree of rotational invariance. This results in a more nearly isotropic numerical dispersion, so that hydrodynamic instabilities will not be amplified from grid effects. Figure 19 depicts the standard "5-point" approximation, in which a grid block $P$ interacts with the four neighbors with which it shares a common interface (solid flow lines). The "9-point" approximation additionally incorporates flow in the diagonal directions (dashed flow lines), which essentially eliminates grid orientation effects in the simulation of steam floods (Yanosik and McCracken, 1979; Coats and Ramesh, 1982; Pruess and Bodvarsson, 1983).

At a temperature of $20^{\circ} \mathrm{C}$, the viscosity of TCE $\left(0.59 \times 10^{-3} \mathrm{Pas}\right)$ is larger than the viscosity of air $\left(1.66 \times 10^{-5} \mathrm{~Pa} . \mathrm{s}\right)$, but smaller than the viscosity of water $\left(1.00 \times 10^{-3}\right.$ Pa.s). Thus displacement of air by TCE has a favorable mobility ratio, while displacement of water has an unfavorable mobility ratio, so that invasion of TCE into an aquifer would be subject to a viscous instability which, however, would not be very strong as the viscosities of TCE and water differ by less than a factor 2 .

There is another kind of hydrodynamic instability which is of much more significance and concern with TCE, namely, the gravitational instability of a denser fiuid (TCE) invading regions with less dense fluids (air, water) from above. This kind of 
instability may lead to strong grid orientation effects in vertical section models. Such effects have been recently studied for the problem of cold water injection into depleted vapor zones in vapor-dominated geothermal reservoirs (Pruess, 1991). Issues of gravitational instability and the associated potential for grid orientation effects in the release of TCE into the vadose zone are entirely analogous to the geothermal injection problem. In order to evaluate these effects we have performed a simulation study that closely parallels the geothermal injection study.

Using the "EOS8" three-phase module of TOUGH2, we have simulated the migration of TCE plumes in vertical section models that employ parallel and diagonal grids, respectively (Figure 20). The grids shown in Figure 20 are meant to illustrate the concepr; in the actual simulations we use a $12 \times 12$ parallel grid of $5 \mathrm{~m} * 5 \mathrm{~m}$ blocks, and a diagonal grid with $7.5 \mathrm{~m}$ long diagonal (5.303 m side length). The flow system is $1 \mathrm{~m}$ thick and the vertical and horizontal dimensions are $60 \mathrm{~m}$ each. The water table is at a depth of $37.5 \mathrm{~m}$; constant pressure conditions are maintained at the right boundary, with all other boundaries being "no flow." TCE is injected in the left uppermost grid block at a rate of $89.68 \times 10^{-6} \mathrm{~kg} / \mathrm{s}$, corresponding to 1 barrel per 30-day month. Simulations were performed for a ten-year period, using parallel and diagonal grids, and 5-point and 9-point differencing schemes. Results for TCE plumes after 10 years are shown in Figure 21 , where shading indicates the presence of NAPL saturations of $1 \%$ or larger. Comparison of the 5-point results for parallel and diagonal grids shows that grid orientation effects are very large. The parallel grid produces a narrow TCE plume that slumps downward, and then spreads along the impermeable bottom of the flow system. In contrast, the diagonal grid gives considerable lateral spreading of the plume. A comparison of the 9point results for both grids shows that grid orientation effects have been essentially completely eliminated.

It is to be emphasized that absence of grid orientation effects does not necessarily mean that physically realistic results have been obtained. It simply means that the anisotropy of numerical dispersion has been reduced in such a way that it will not amplify a hydrodynamic instability which is present in the flow system. Physically realistic modeling of the migration of a NAPL plume under conditions of a gravitationally induced hydrodynamic instability requires proper description of all physical mechanisms that will affect plume dispersion in a heterogeneous medium. In the absence of heterogeneity, the downward slumping predicted by the parallel 5-point grid is actually correct, because this differencing scheme produces numerical dispersion only in the vertical direction (Pruess, 1991). A lateral (transverse) spreading of the plume can arise from formation heterogeneity, such as presence of clay lenses and layers, as was demonstrated in the numerical 
simulations discussed above. Although diagonal grids and higher-order differencing methods produce more neardy isotropic dispersion effects, there is no reason to expect the lateral dispersion in these differencing schemes to adequately approximate true physical dispersion effects in a heterogeneous medium. We believe that the 5-point parallel differencing scheme is acceptable when heterogeneities are modeled in explicit detail. The 5-point diagonal as well as parallel and diagonal 9-point schemes introduce a lateral numerical dispersion that in detailed molels with explicit representation of heterogeneity would arise from flow diversion due to the heterogeneities. A physically realistic representation of the dispersive effects of heterogeneity in continuum-based numerical models of multiphase flow has yet to be developed.

\section{Concluding Remarks}

We have presented engineering estimates as well as numerical simulations for the multiphase processes that arise from TCE release in the vadose zone. Although the basic physico-chemical processes of flow and phase partitioning are simple and well understood, their analysis is complicated by numerous coupled effects and by the ever-present and always imperfectly known heterogeneity of geologic media. Our simulations help to explain the preferential association of TCE contamination with clays that was observed at the Savannah River site (Eddy et al., 1991). Buoyancy flow in the gas phase was found to have a potential for spreading contamination from localized sources over large areas.

Although a large amount of data is available for the site, some of the most important parameters affecting TCE behavior in the vadose zone, such as three-phase relative permeabilities and capillary pressures, are poorly known. Presently available simulation techniques can cope with the highly nonlinear multiphase processes affecting TCE migration in the subsurface. Further research is needed to develop a better understanding of the behavior of multiphase fluid mixtures in heterogeneous systems.

In future projects dealing with subsurface contamination, it would seem beneficial to integrate simulation-based performance assessment modeling as early and closely as possible with the site characterization, monitoring, and remediation activities.

\section{Acknowledgement}

This work was supported through the Office of Technology Development, U.S. Department of Energy, under contract DE-AC03-76SF00098. The author appreciates the collaboration of managers and technical staff of the Westinghouse Savannah River 
Company, and acknowledges helpful technical discussions with Dwayne Chesnut, Lawrence Livermore National Laboratory. Thanks are due to $\mathrm{Y}$. Tsang for a critical review of the manuscript.

\section{References}

Brand, C. W., Heinemann, J. E. and Aziz, K., The Grid Orientation Effect in Reservoir Simulation, paper SPE-21228, presented at Society of Petroleum Engineers Eleventh Symposium on Reservoir Simulation, Anaheim, CA, February 1991.

Coats, K. H., Reservoir Simulation: State of the Art, J. Pet. Tech., 1633-1642, August 1982.

Coats, K. H., George, W. D., Chu, C. and Marcum, B. E., Three-Dimensional Simulation of Steamflooding, Soc. Pet. Eng. J., 573-592, December 1974.

Coats, K. H. and Ramesh, A. B., Effects of Grid Type and Difference Scheme on Pattern Steamflood Simulation Results, paper SPE-11079, presented at the 57th Annual Fall Technical Conference and Exhibition of the Society of Petroleum Engineers, New Orleans, La., September 1982.

Colven, W. P., Boone, L. F., Horvath, J. G. and Lorenz, R., Effectiveness of the M-Area Ground Water Remedial Action Program, E. I. DuPont de Nemours and Company, Report DPSP-87-26, Aiken, South Carolina, February 1987.

Eddy, C. A., Looney, B. B., Dougherty, J. M., Hazen, T. C. and Kaback, D. S., Characterization of the Geology, Geochemistry, Hydrology, and Microbiology of the InSitu Air Stripping Demonstration Site at The Savannah River Site (U), Westinghouse Savannah River Company, Report WSRC-RD-91-21, Aiken, South Carolina, May 1991.

Falta, R. W., Javandel, I., Pruess, K. and Witherspoon, P. A., Density-Driven Flow of Gas in the Unsaturated Zone due to the Evaporation of Volatile Organic Compounds, Water Resources Research, 25, (10), 2159-2169, 1989.

Falta, R. W., Pruess, K., Javandel, I. and Witherspoon, P. A., Numerical Modeling of Steam Injection for the Removal of Nonaqueous Phase Liquids from the Subsurface. 1. Numerical Formulation, Water Resources Research, 28, (2), 433-449, $1992 \mathrm{a}$.

Falta, R. W., Pruess, K., Javandel, I. and Witherspoon, P. A., Numerical Modeling of Steam Injection for the Removal of Nonaqueous Phase Liquids from the Subsurface. 2. Code Validation and Application, Water Resources Research, 28, (2), $451-465,1992 b$.

Falta, R. W. and Pruess, K., STMVOC User's Guide, Lawrence Berkeley Laboratory Report LBL-30758, June 1991. 
Parker, J. C., Lenhard, R. J. and Kuppusamy, T. A Parametric Model for Constitutive Properties Governing Multiphase Flow in Porous Media, Water Resources Research, 23, (4), 618-624, 1987.

Pruess, K., TOUGH User's Guide, Nuclear Regulatory Commission, Report NUREG/CR-4645, June 1987 (also Lawrence Berkeley Laboratory Report LBL20700, Berkeley, CA., June 1987).

Pruess, K, TOUGH2 - A General-Purpose Numerical Simulator for Multiphase Fluid and Heat Flow, Lawrence Berkeley Laboratory Report LBL-29400, May 1991.

Pruess, K., Grid Orientation and Capillary Pressure Effects in the Simulation of Water Injection into Depleted Vapor Zones, Geothermics, 20, (5/6), 257-277, 1991.

Pruess, K. and Bodvarsson, G. S., A Seven-Point Finite Difference Method for Improved Grid Orientation Performance in Pattern Steam Floods, paper SPE-12252, presented at the Seventh Society of Petroleum Engineers Symposium on Reservoir Simulation, San Francisco, 1983.

Stone, H. L., Probability Model for Estimating Three-Phase Relative Permeability, Trans. SPE of AIME, 249, 214-218, 1972.

Todd, M. R., O'Dell, P. M. and Hirasaki, G. J., Methods for Increased Accuracy in Numerical Reservoir Simulators, Soc. Pet. Eng. J., 515-530, December 1972.

Tsang, Y. W. and Pruess, K., Preliminary Studies of Gas Phase Flow Effects and Moisture Migration at Yucca Mountain, Lawrence Berkeley Laboratory Report LBL 28819, Berkeley, CA, 1989.

Yanosik, J. L. and McCracken, T. A., A Nine-Point, Finite Difference Reservoir Simulator for Realistic Prediction of Adverse Mobility Ratio Displacements, Soc. Pet. Eng. J., 253-262, August 1979. 

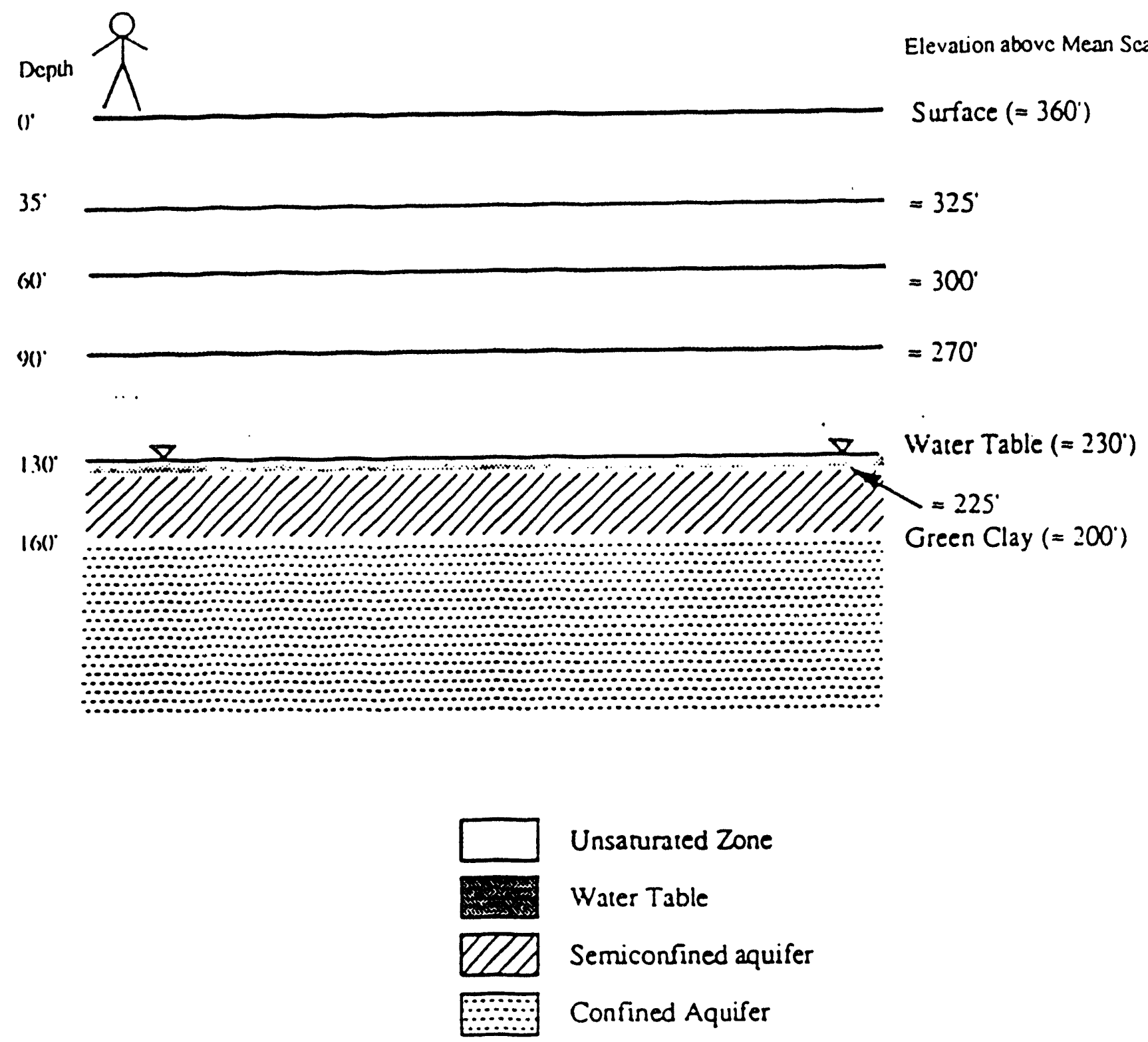

Figure 1 Schematic diagram showing relationship ixetwien clay layers and hydrologic featurcs. (Aficr Figure 3.2. $|4|$.) 


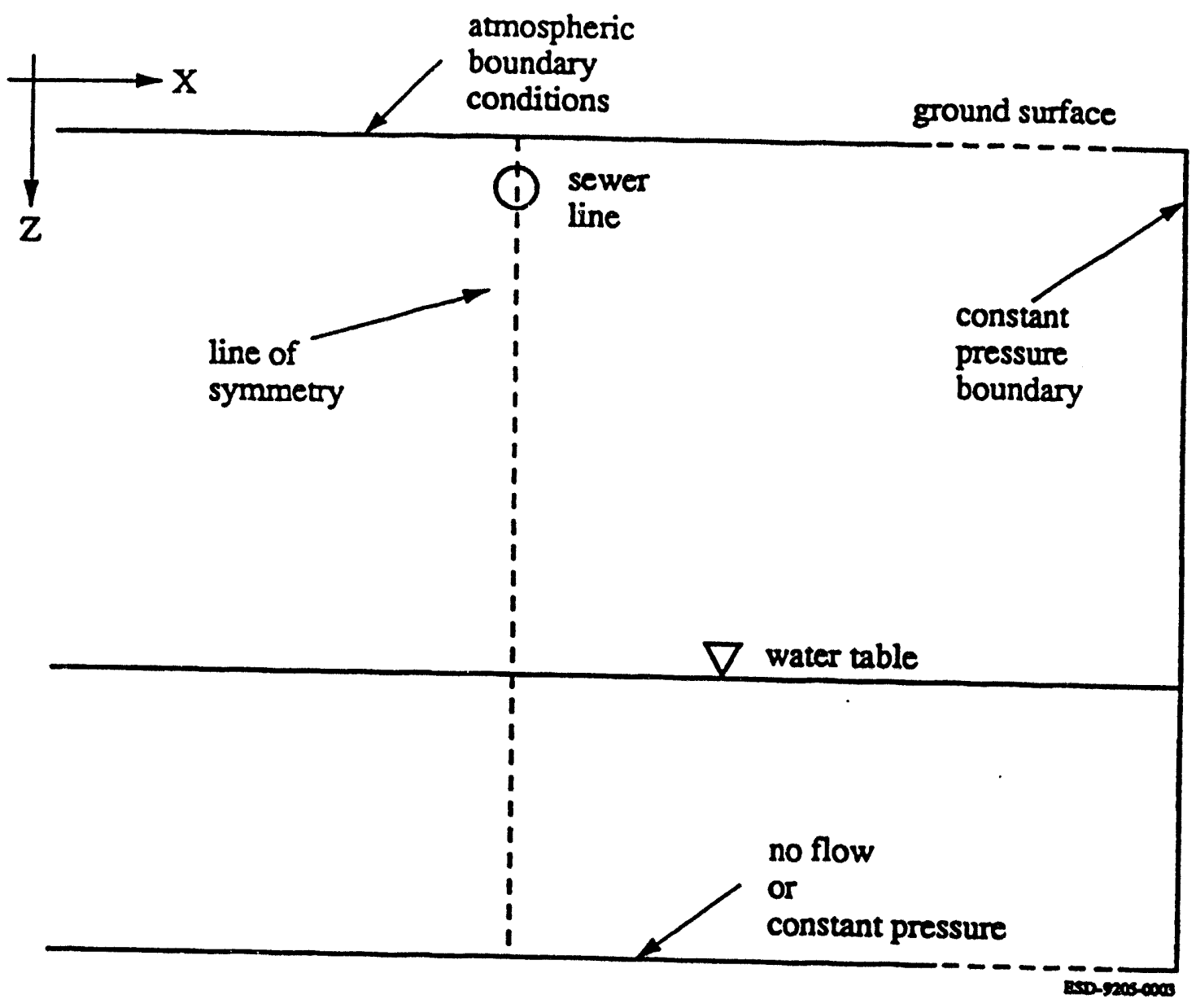

Figure 2. Schematic of Model System 


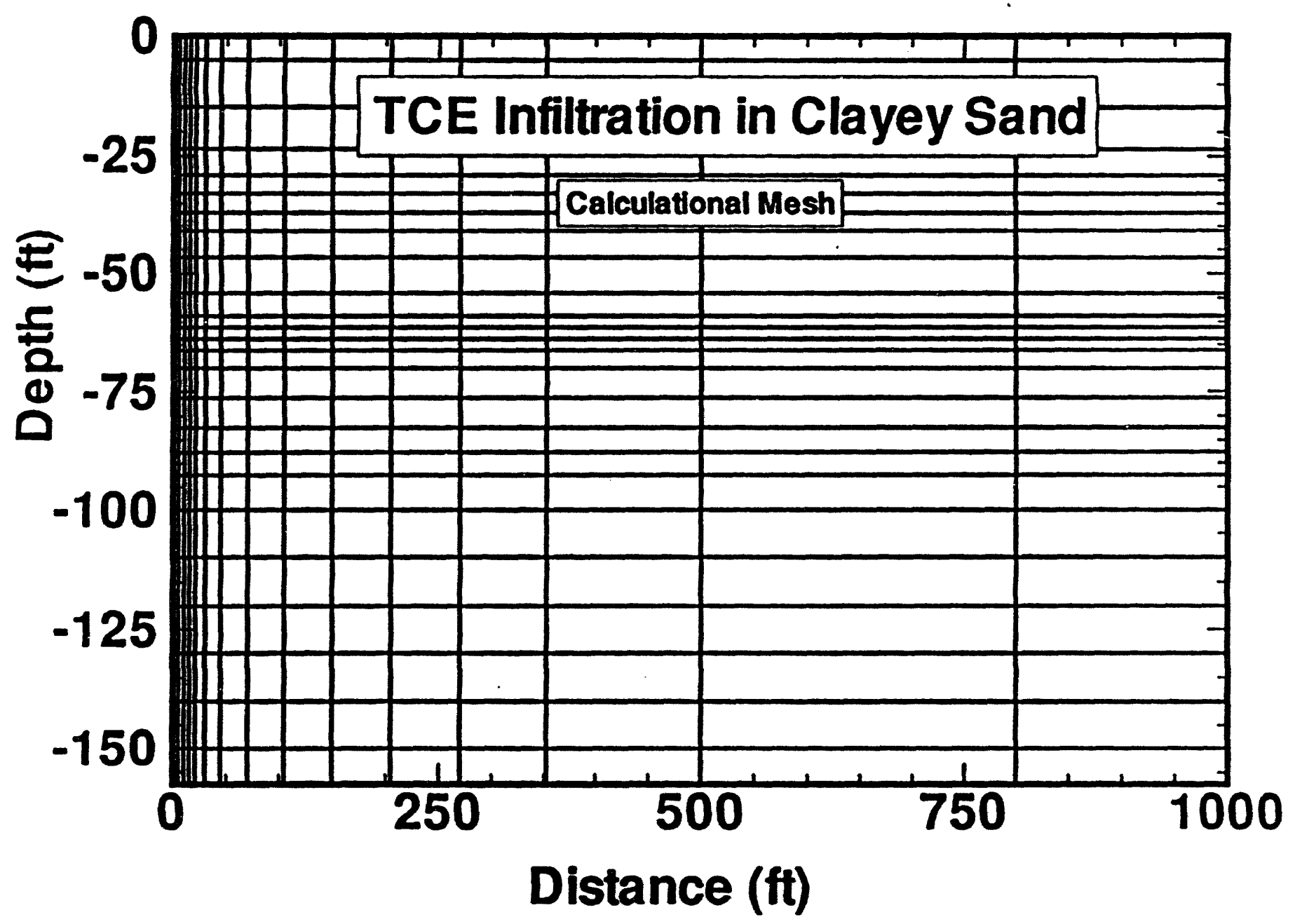




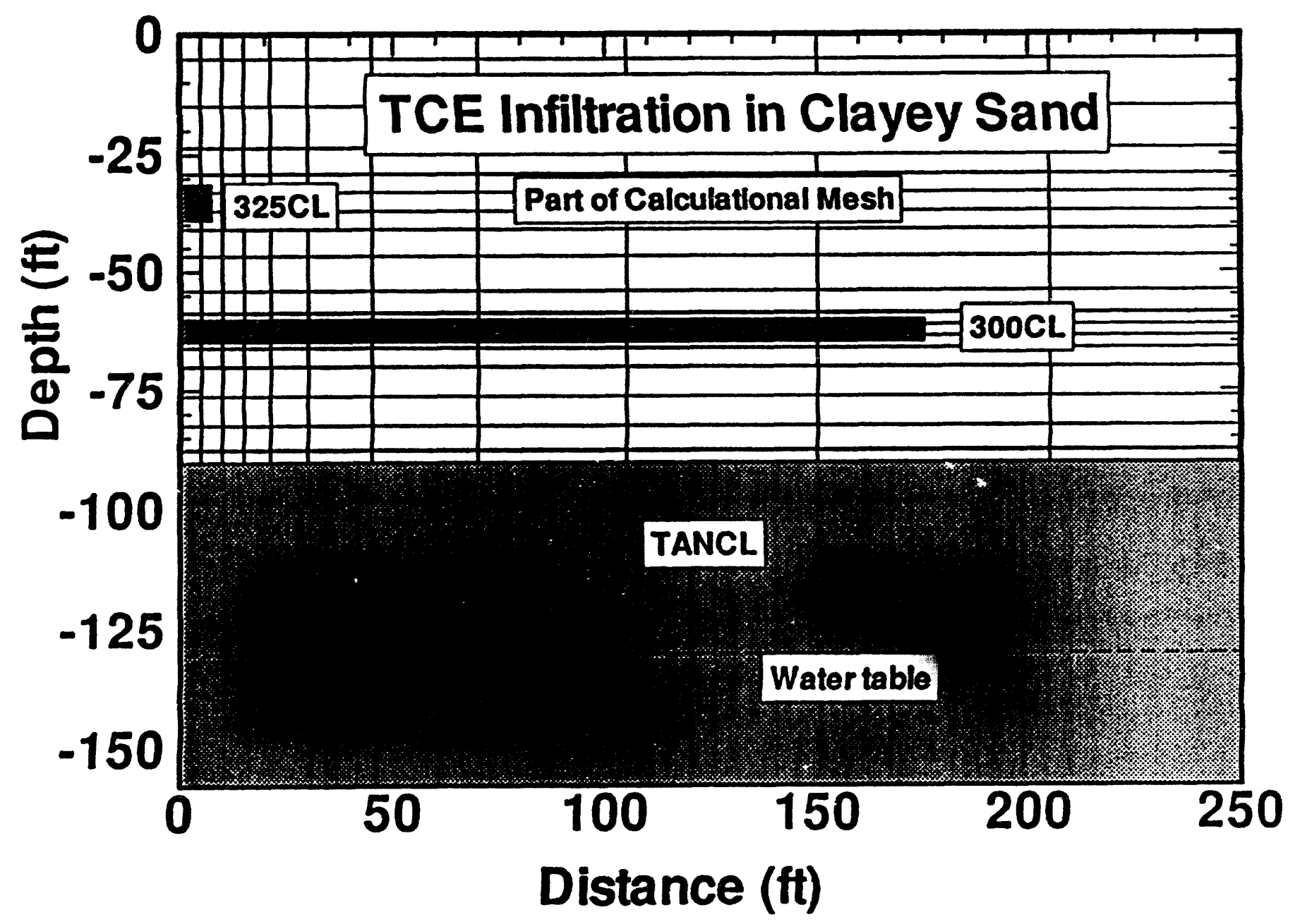




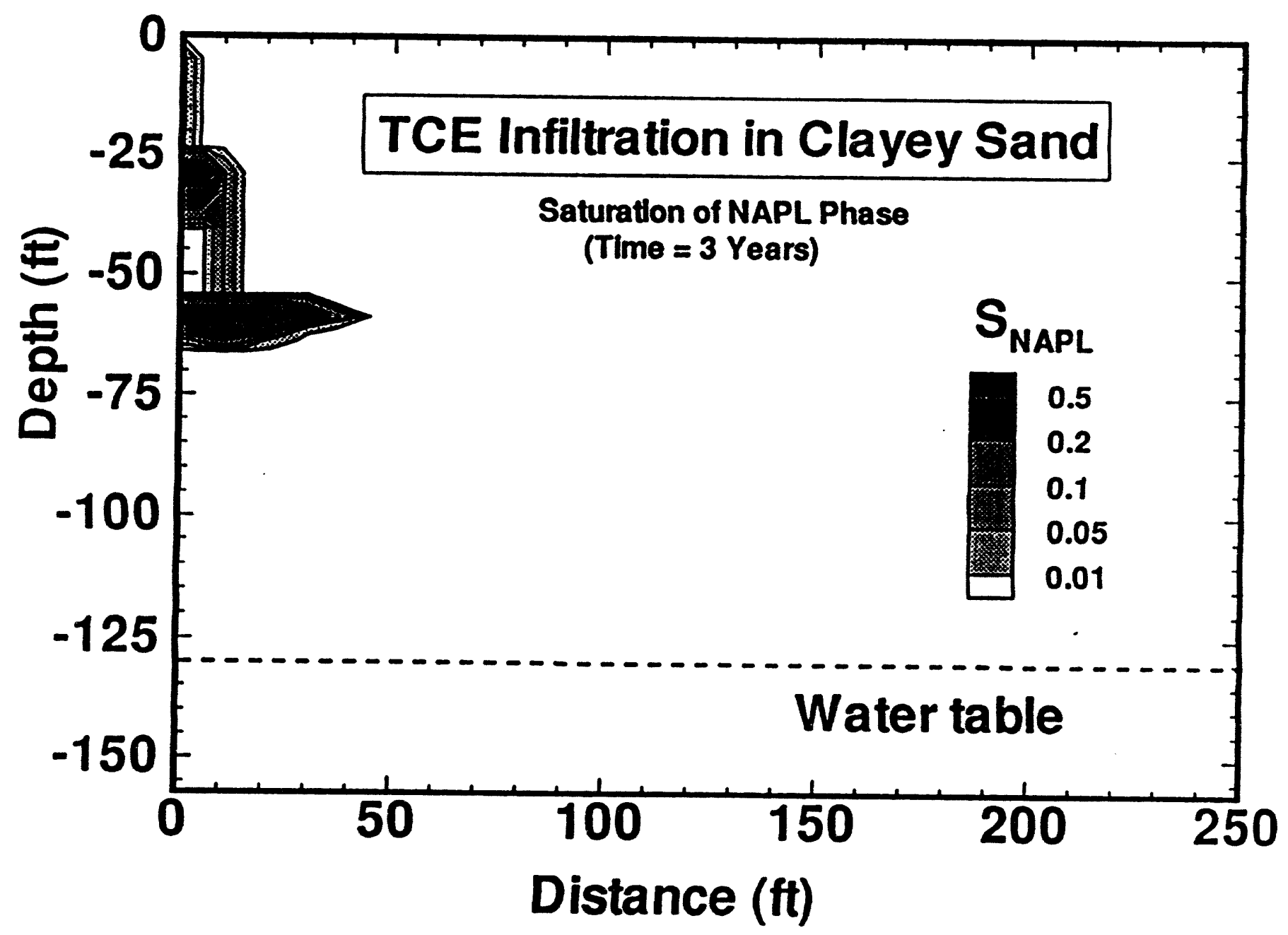




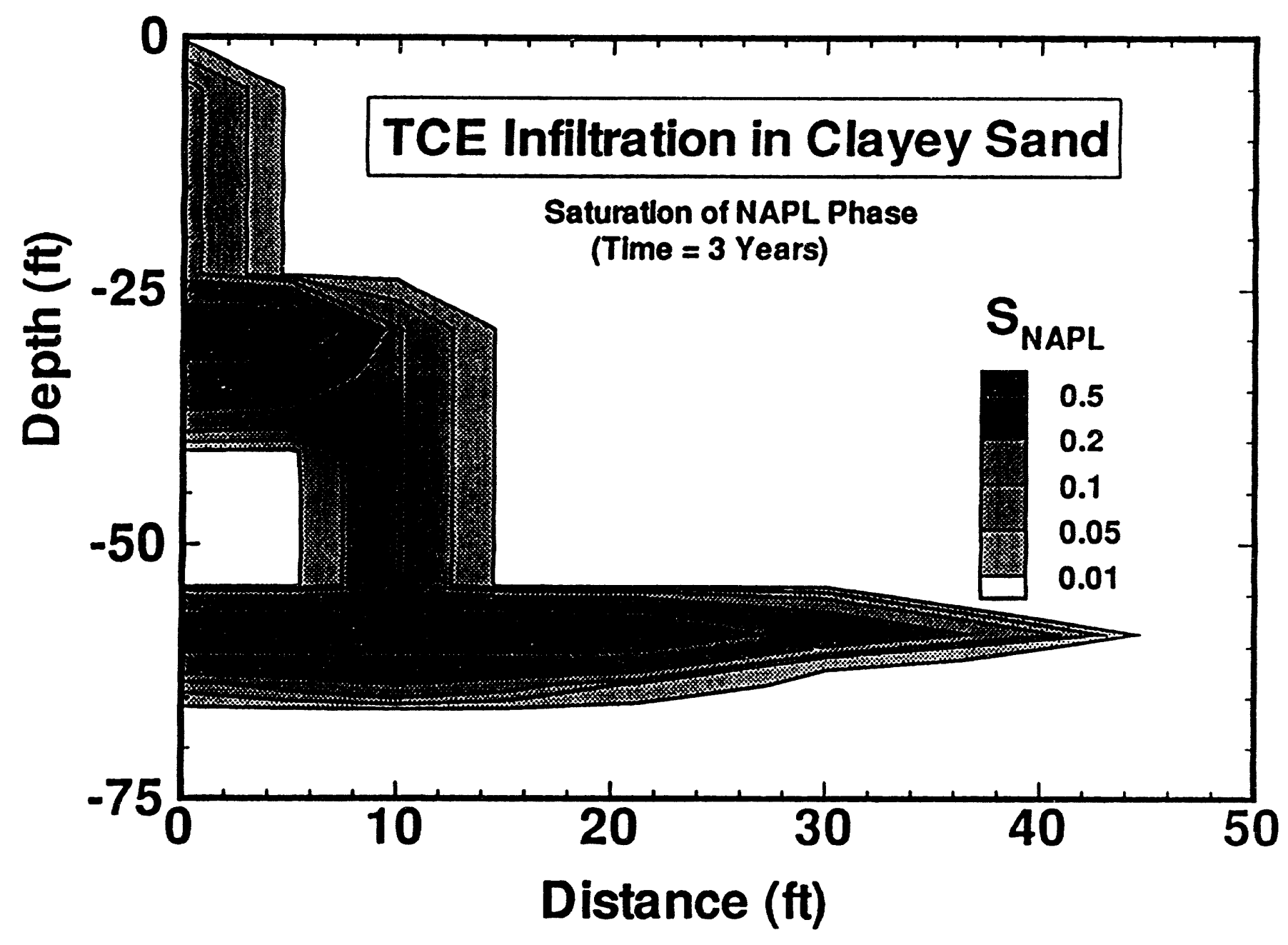




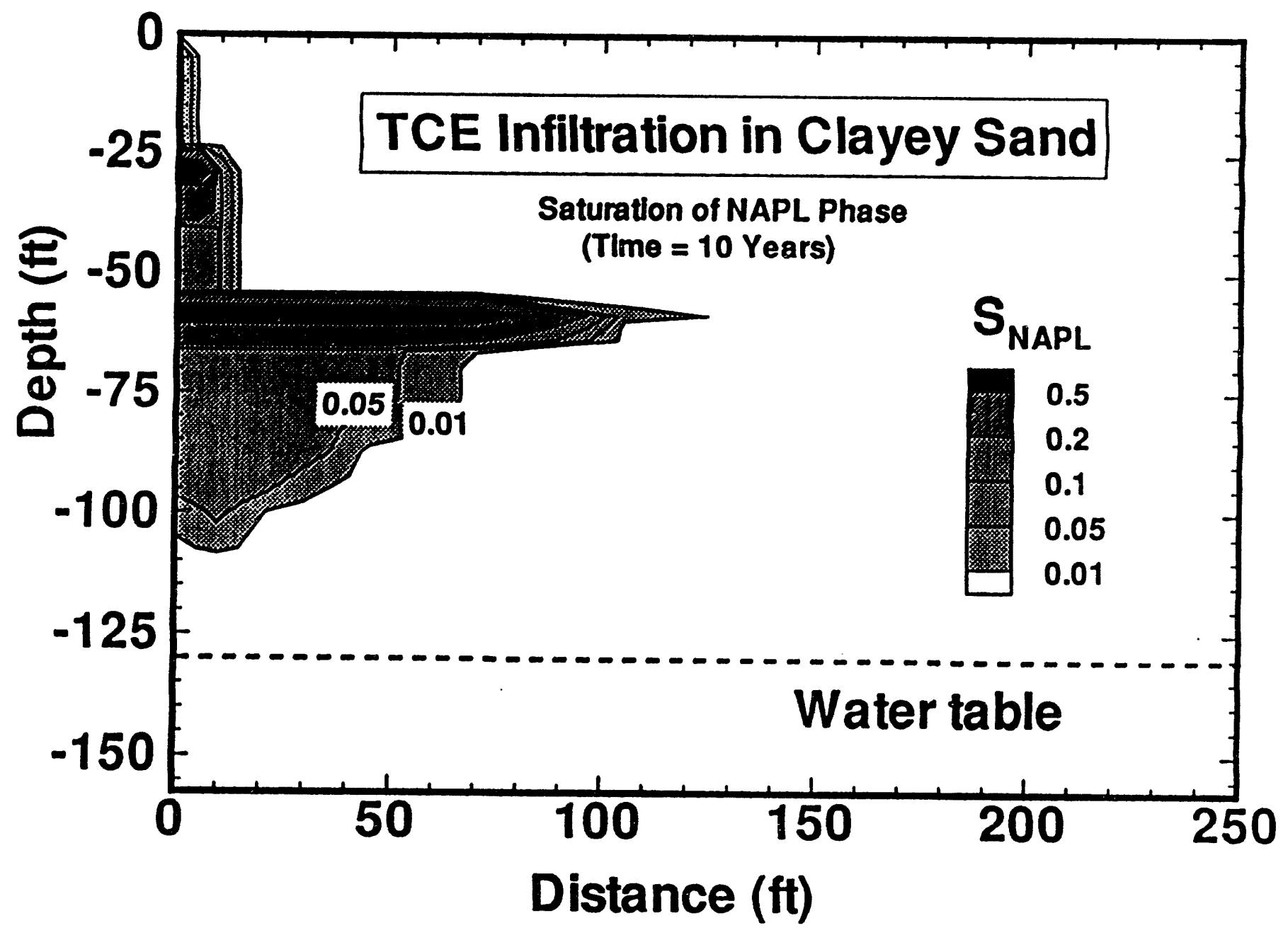




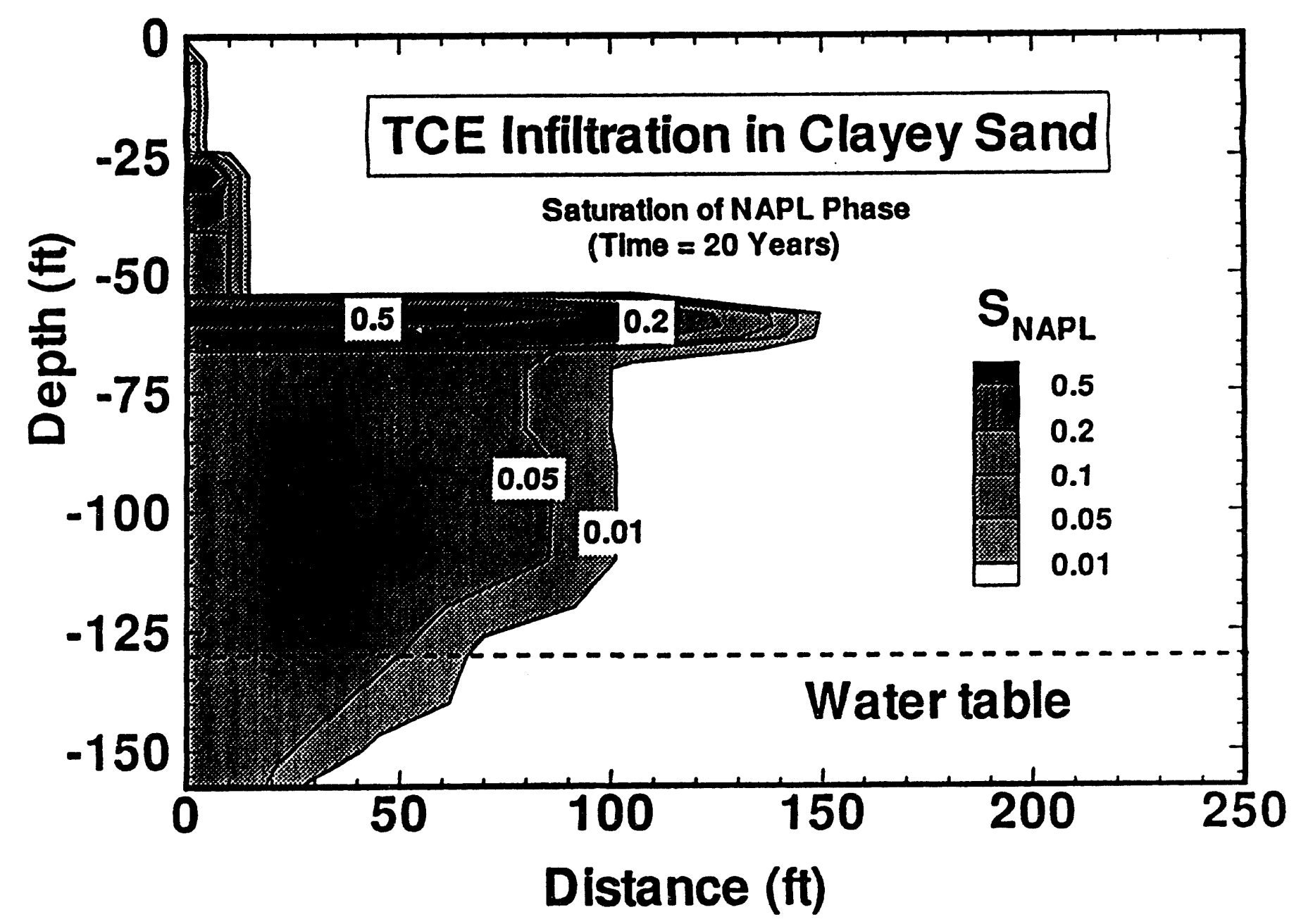




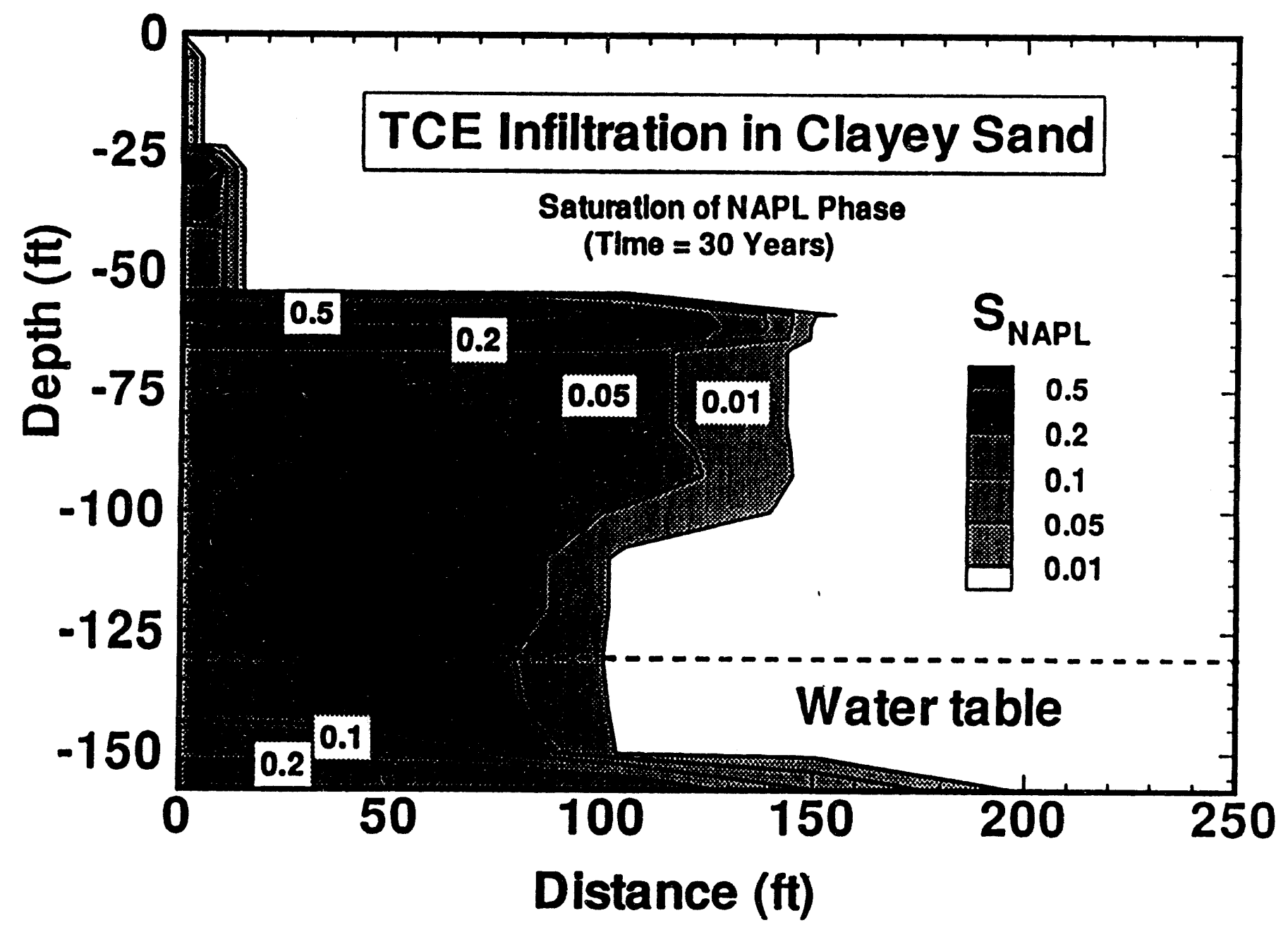




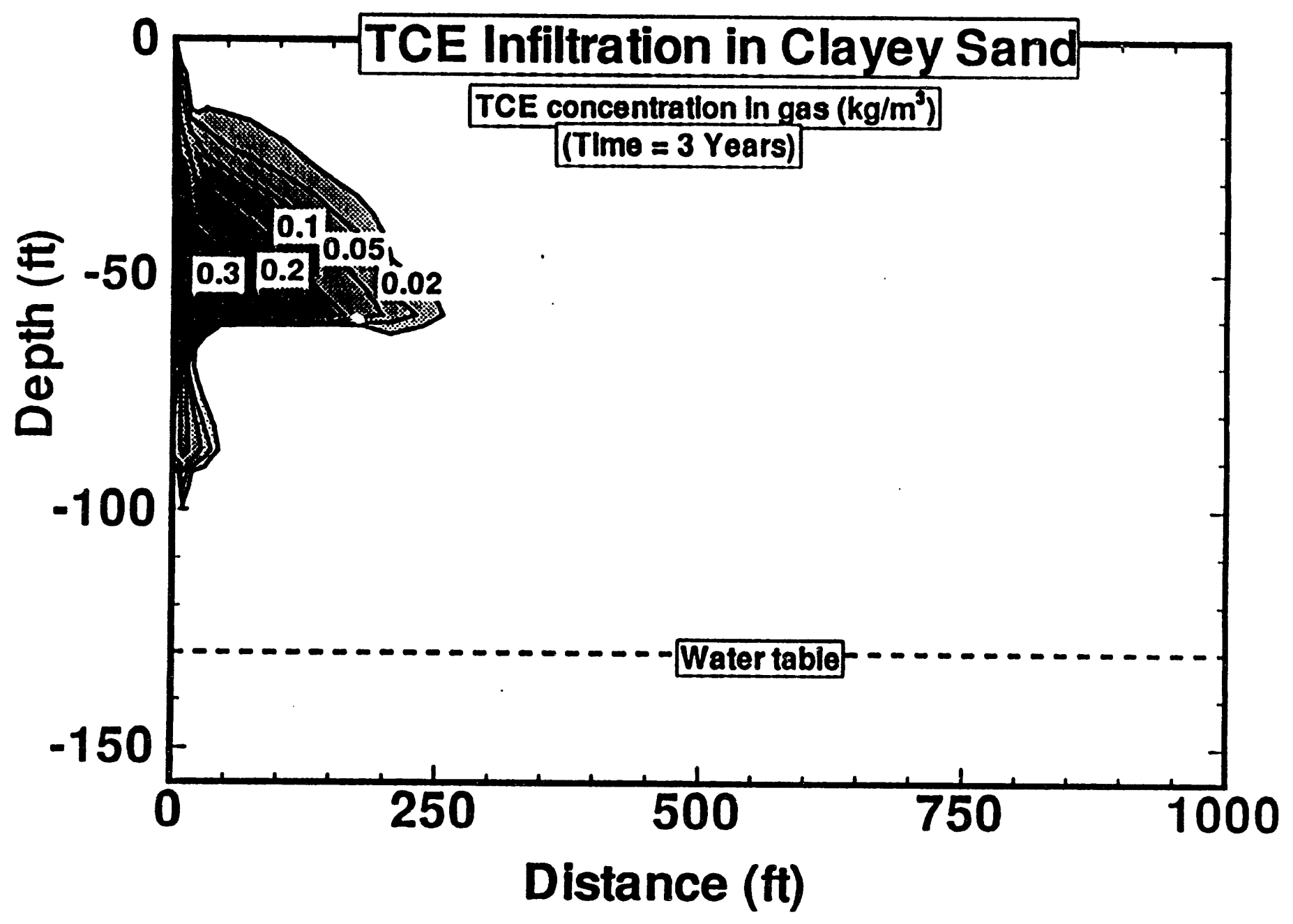




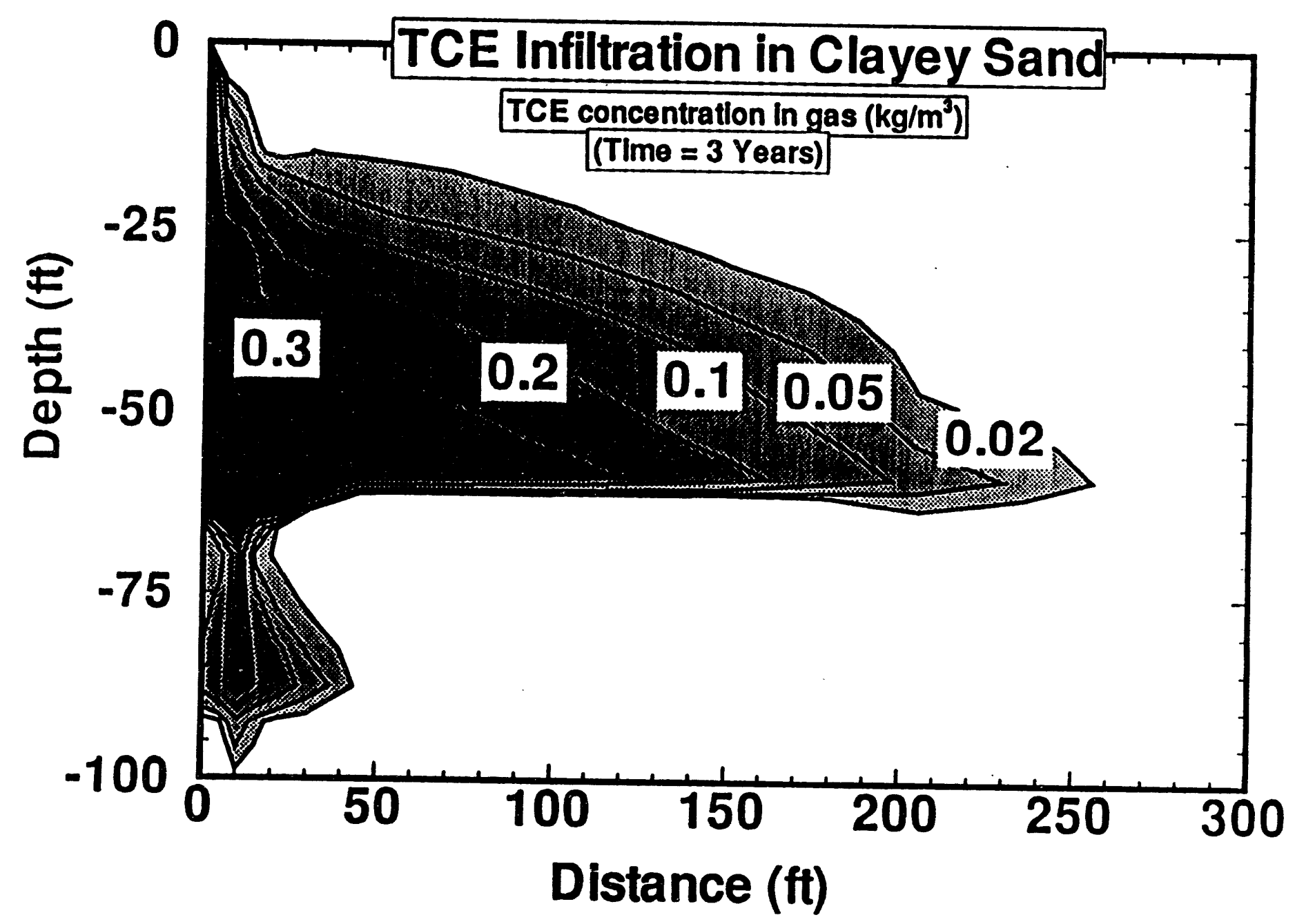




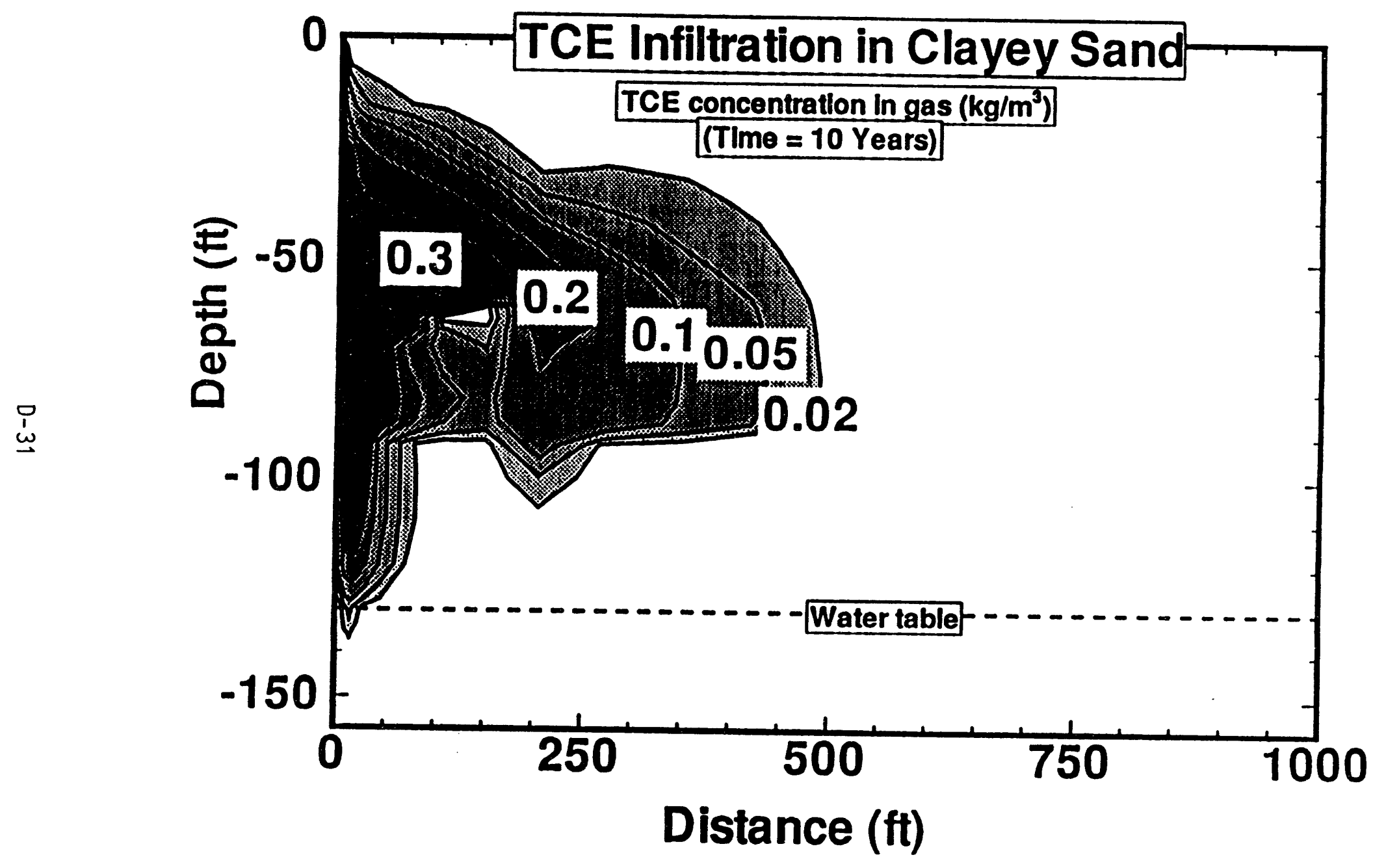




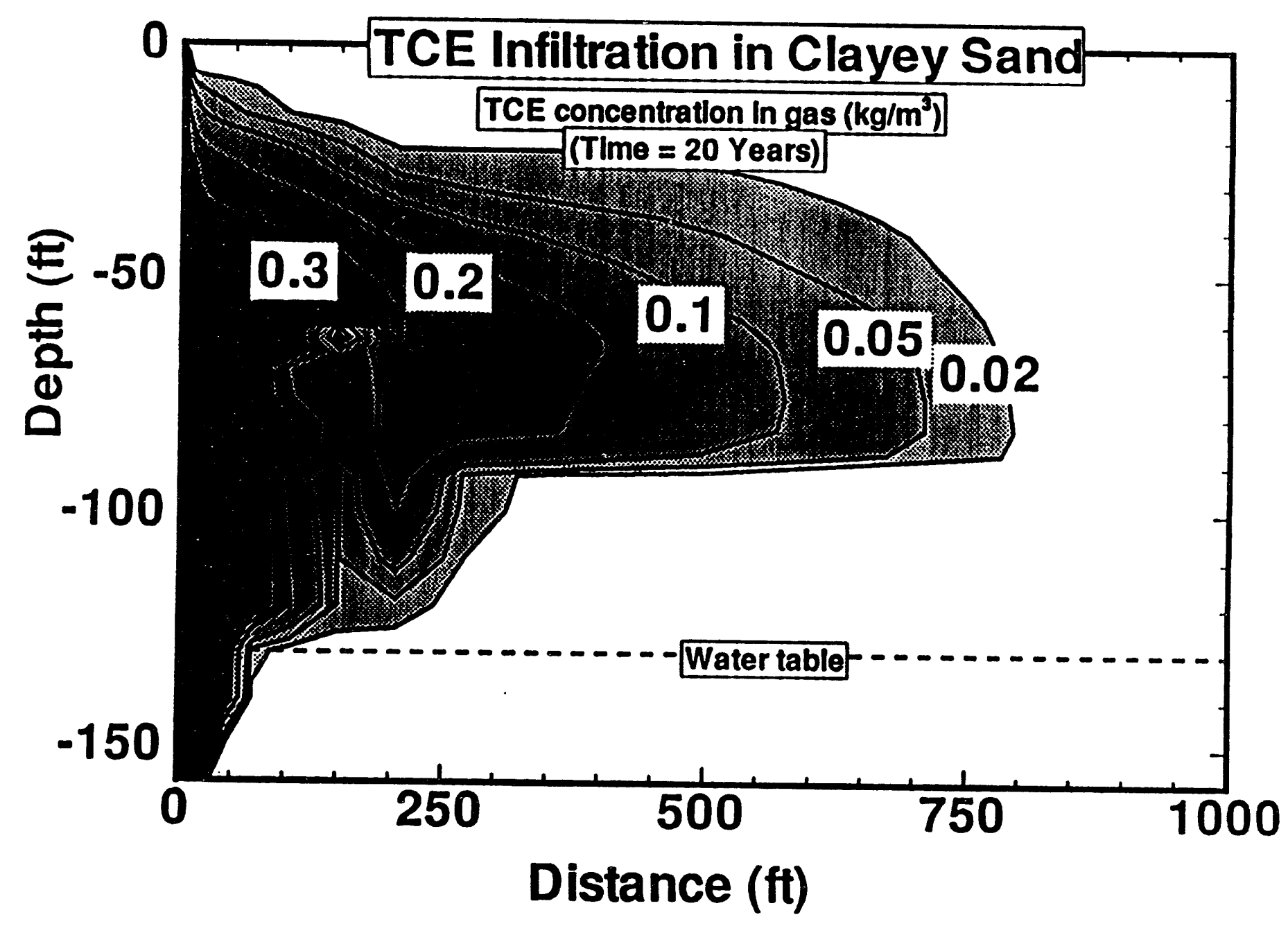




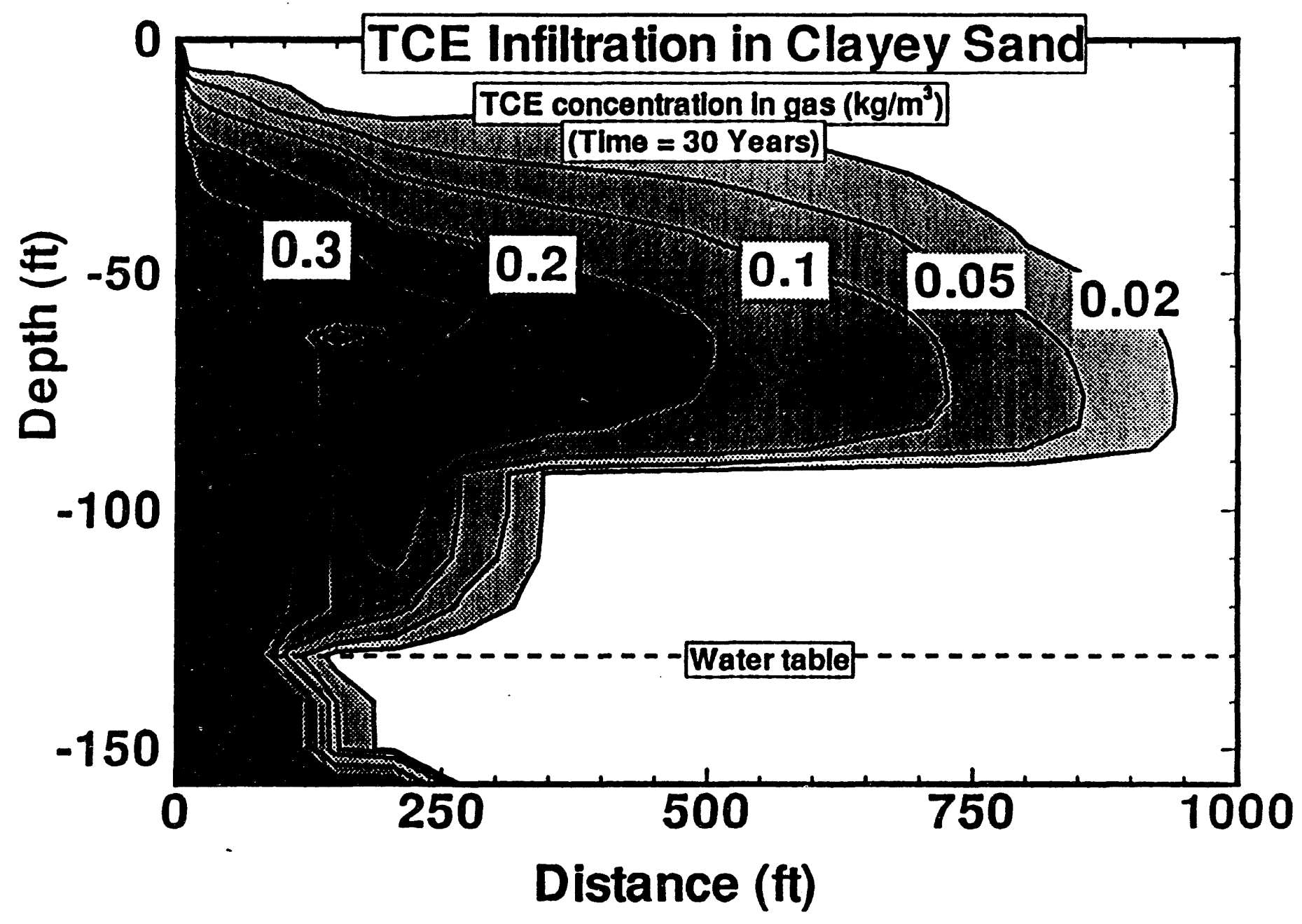




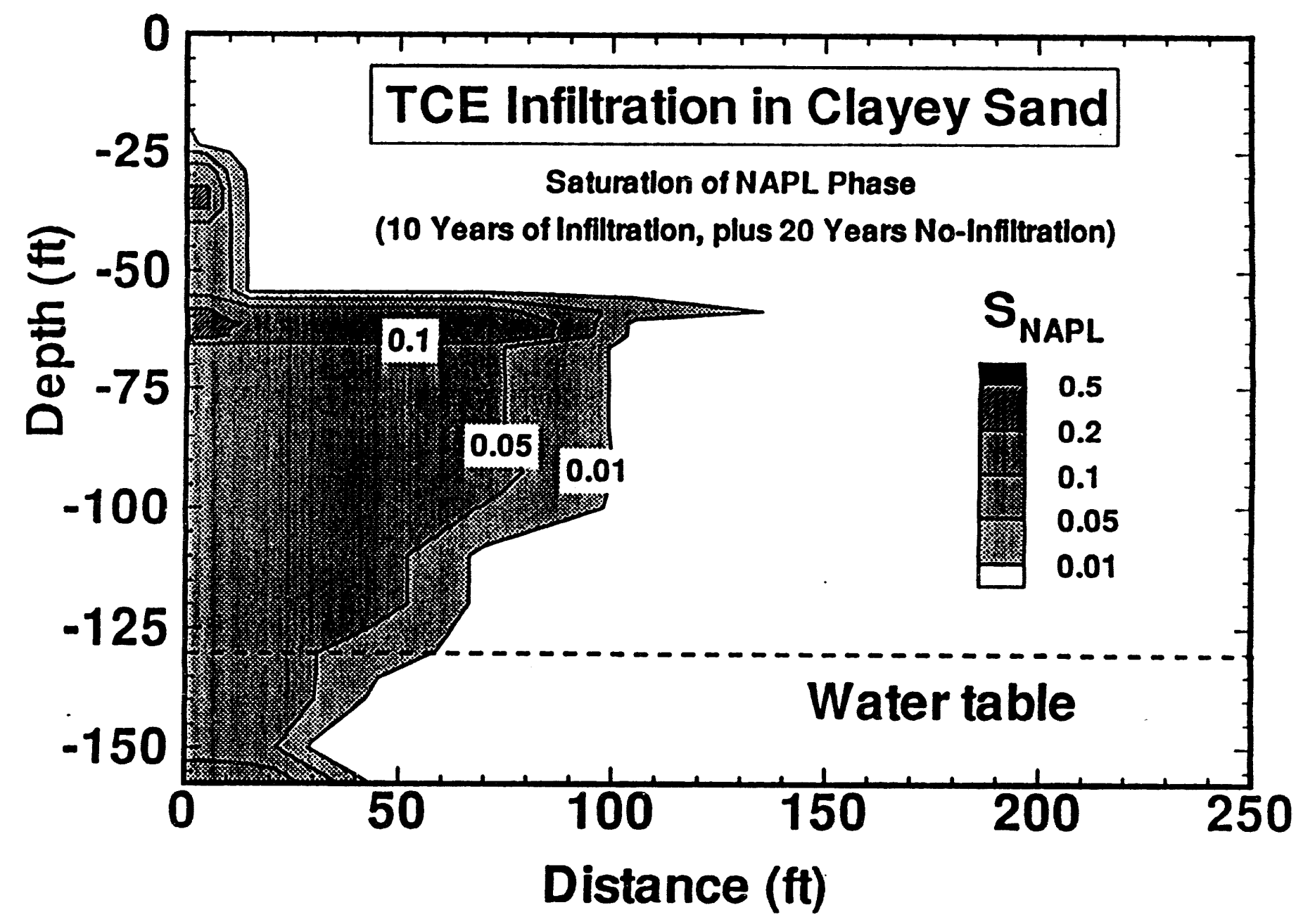




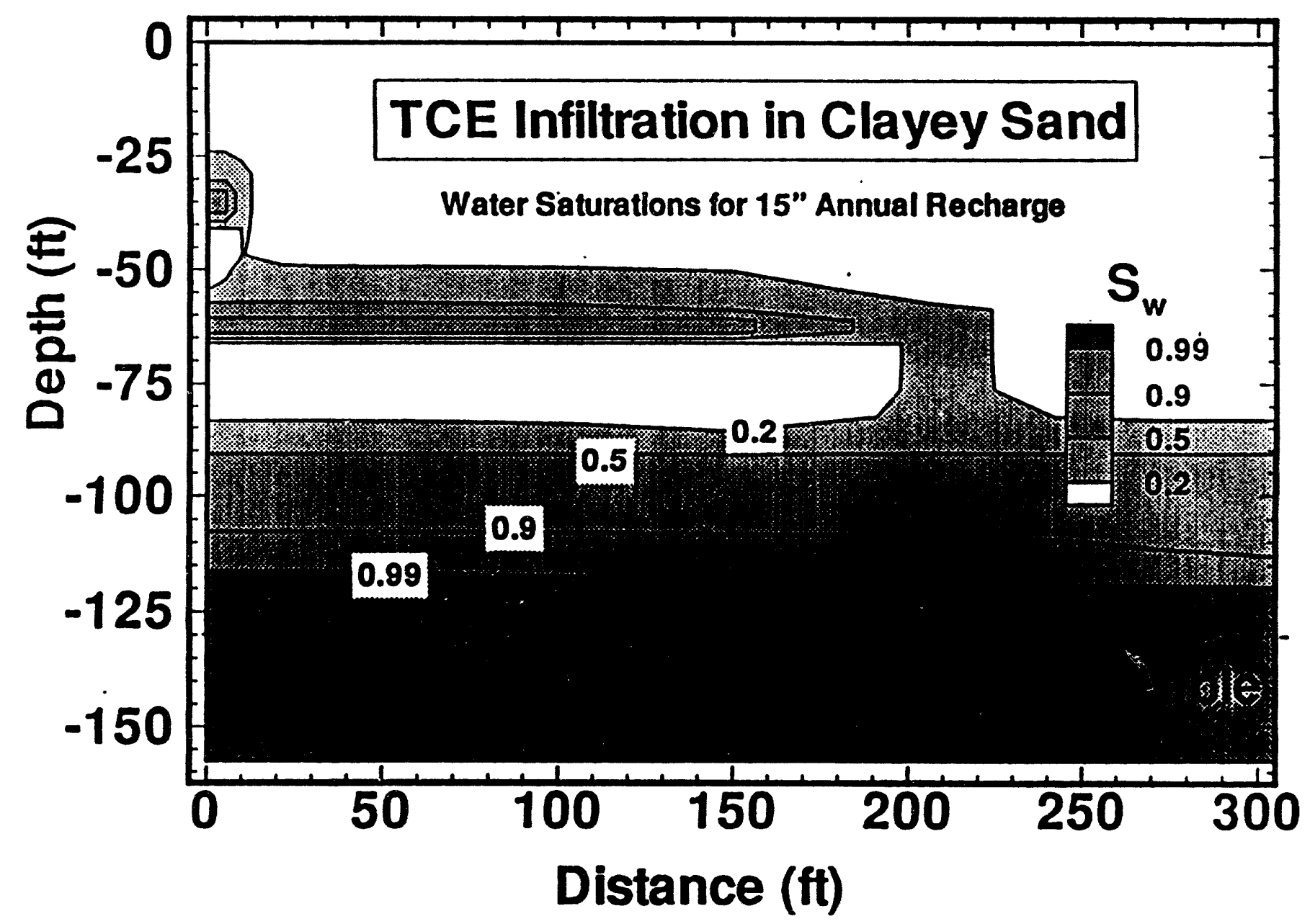




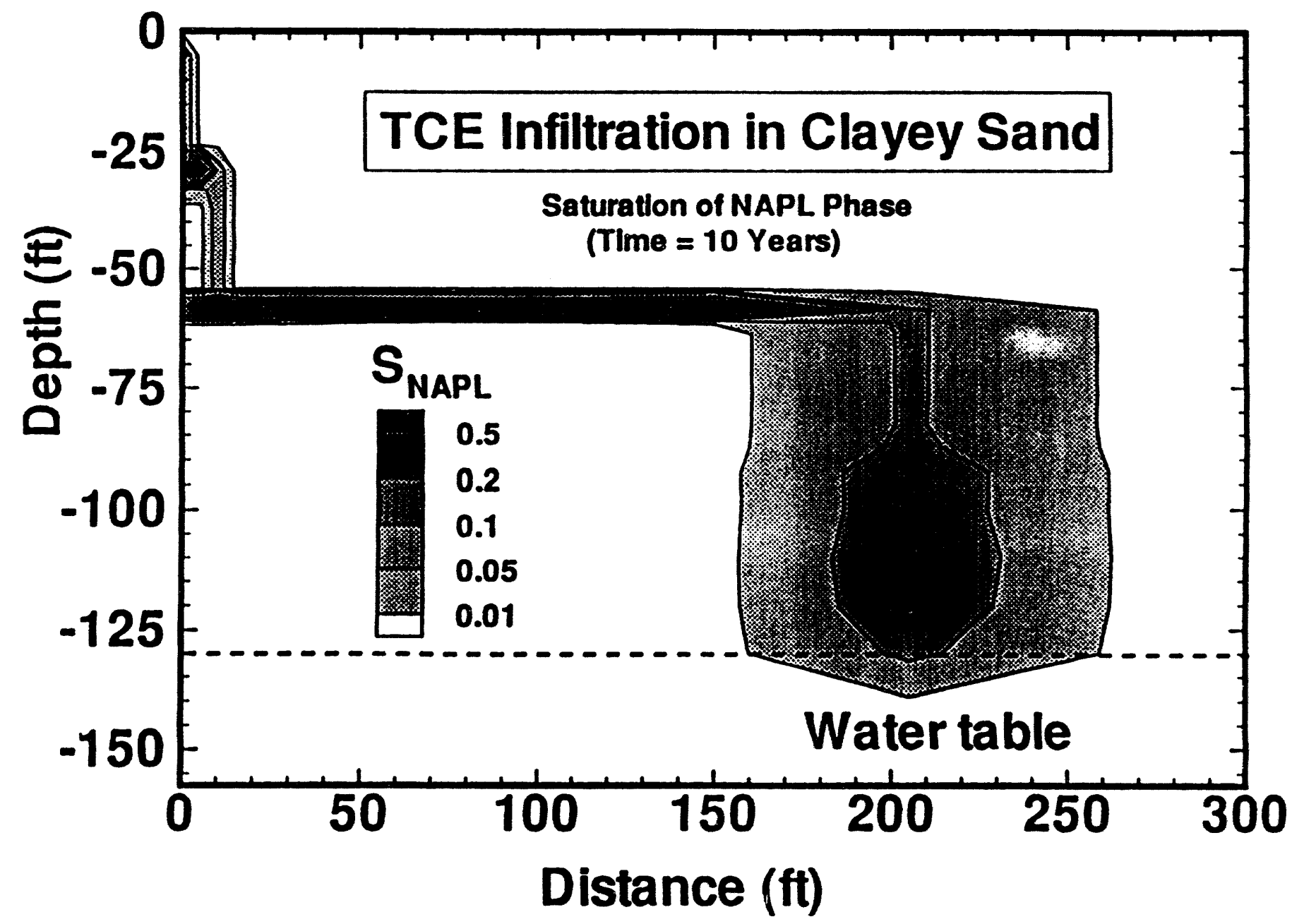




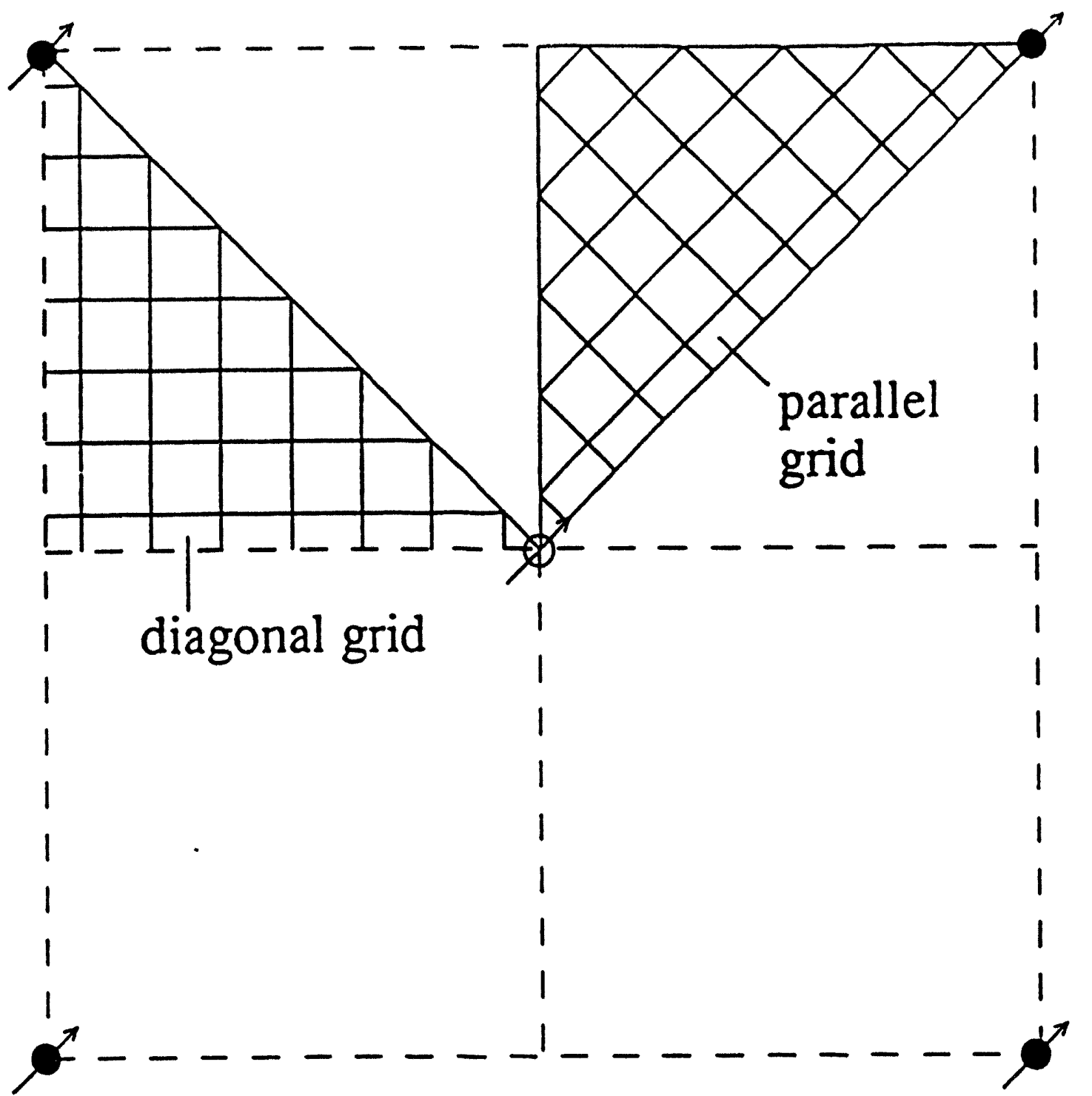

\section{$\varnothing$ Injection Well \\ $\sigma^{\prime \prime}$ Production Well}

XBL $911-103$

Figure 3. Five-spot well pattern with parallel and diagonal grids for modeling a 1/8 symmetry domain. 



\section{columns}

\begin{tabular}{|c|c|c|c|c|c|c|c|}
\hline & 2 & 3 & 4 & 5 & 6 & 7 & 8 \\
\hline rows 1 & • & - & - & - & - & - & • \\
\hline 2 & • & - & - & - & - & - & • \\
\hline 3 & • & - & • & • & - & • & • \\
\hline 4 & $\bullet$ & - & - & - & - & - & - \\
\hline 5 & - & - & - & - & • & • & - \\
\hline 6 & • & - & - & • & - & - & . \\
\hline
\end{tabular}

XBI $911-98$

\section{columns}

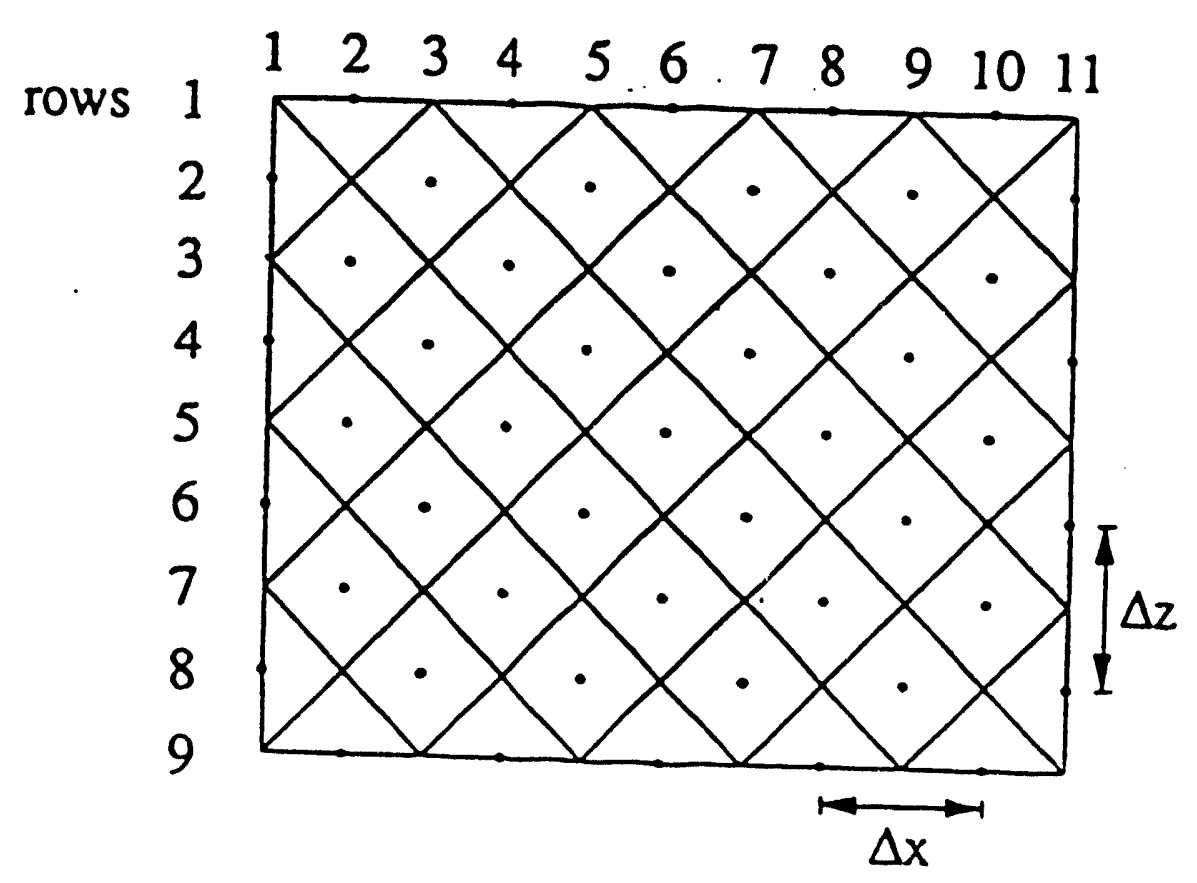

XBL 912-97

Figure 5. Schematic of parallel and diagonal grids used for modeling injection in 2-D
vertical section. 

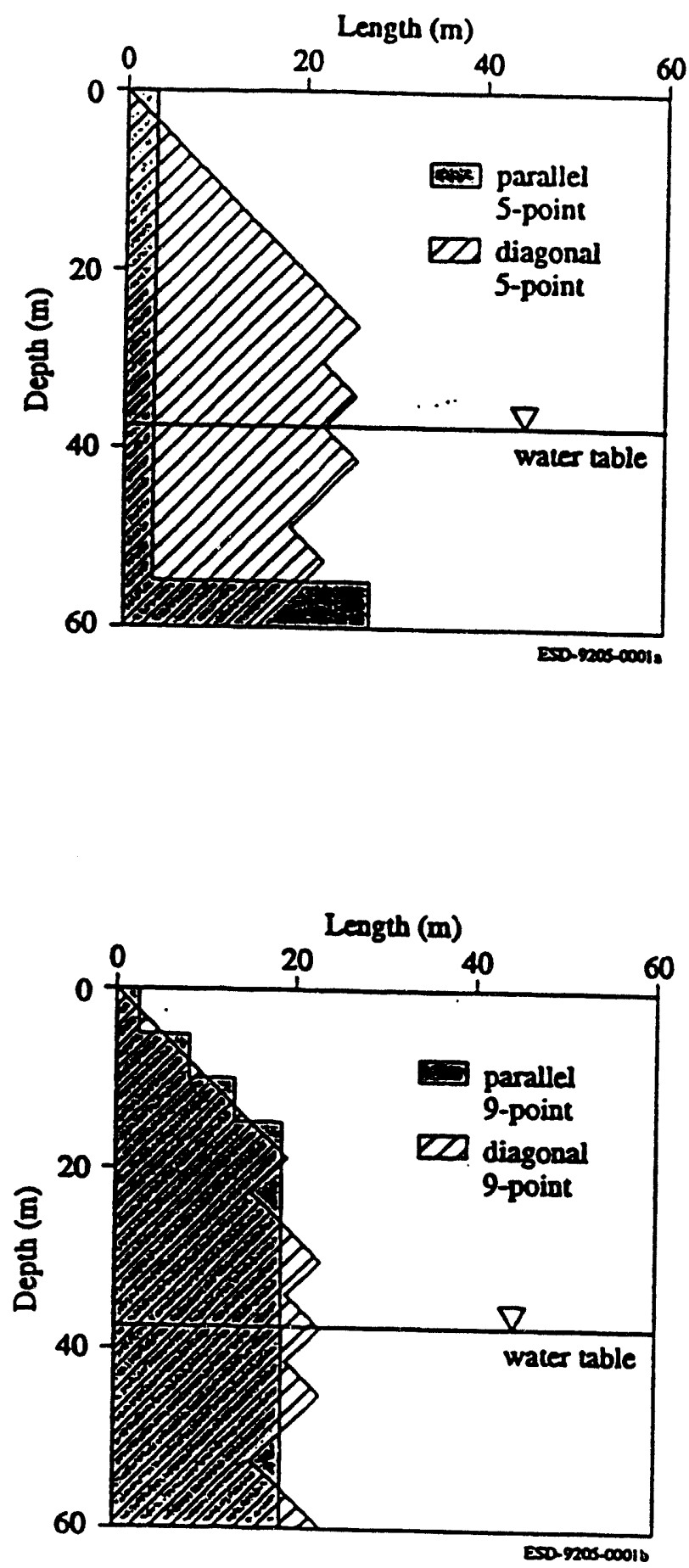


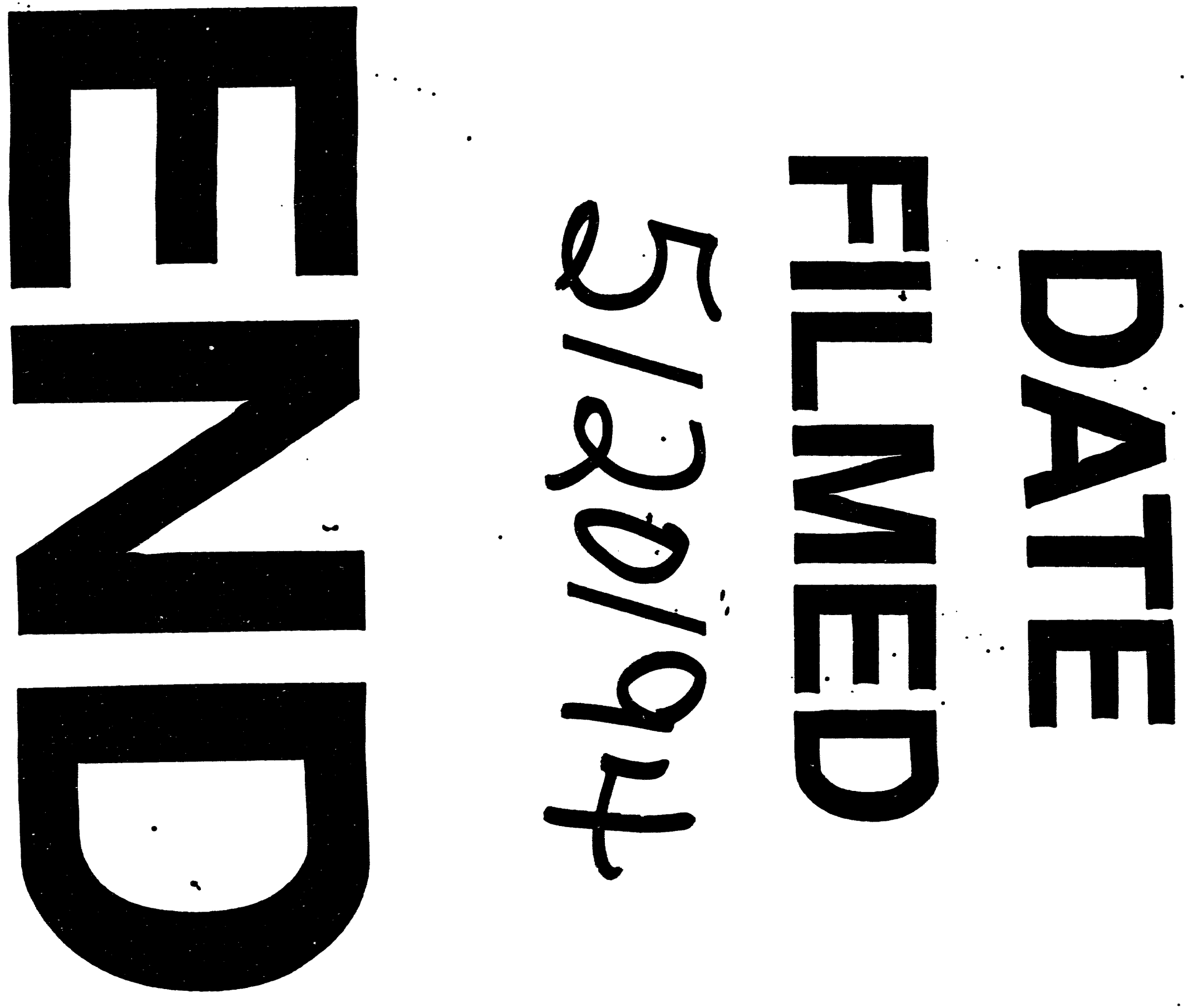

